Scientific Publications of the American Museum of Natural History

AMERICAN MUSEUM NOVITATES

BULletin of the AMERICAN MUSEUM of NATURAL History

anthropological papers of the american Museum of Natural History

Publications COMmittee

ROBERT S. VOSS, CHAIR

BOARD OF EDITORS

JiN MENG PALEONTOLOGY

LORENZO Prendini, INVERTEBRATE ZOOLOGY

ROBERT S. VOSS, VERTEBRATE ZOOLOGY

Peter M. WHiteley, ANTHROPOLOGY

MANAGING EDITOR

MARY KNIGHT

Submission procedures can be found at http://research.amnh.org/scipubs

All issues of Novitates and Bulletin are available on the web from http://digitallibrary.amnh.org/dspace

Order printed copies from http://www.amnhshop.com or via standard mail from: American Museum of Natural History_-Scientific Publications

Central Park West at 79th Street

New York, NY 10024

(@) This paper meets the requirements of ANSI/NISO Z39.48-1992 (permanence of paper).

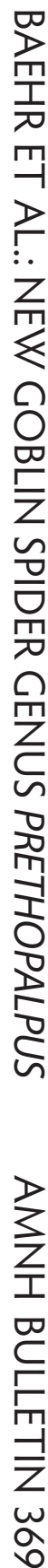

THE NEW AUSTRALASIAN GOBLIN

SPIDER GENUS PRETHOPALPUS (ARANEAE, OONOPIDAE)

\author{
BARBARA C. BAEHR, MARK S. HARVEY, \\ MATTHIAS BURGER, AND MARCO THOMA
}

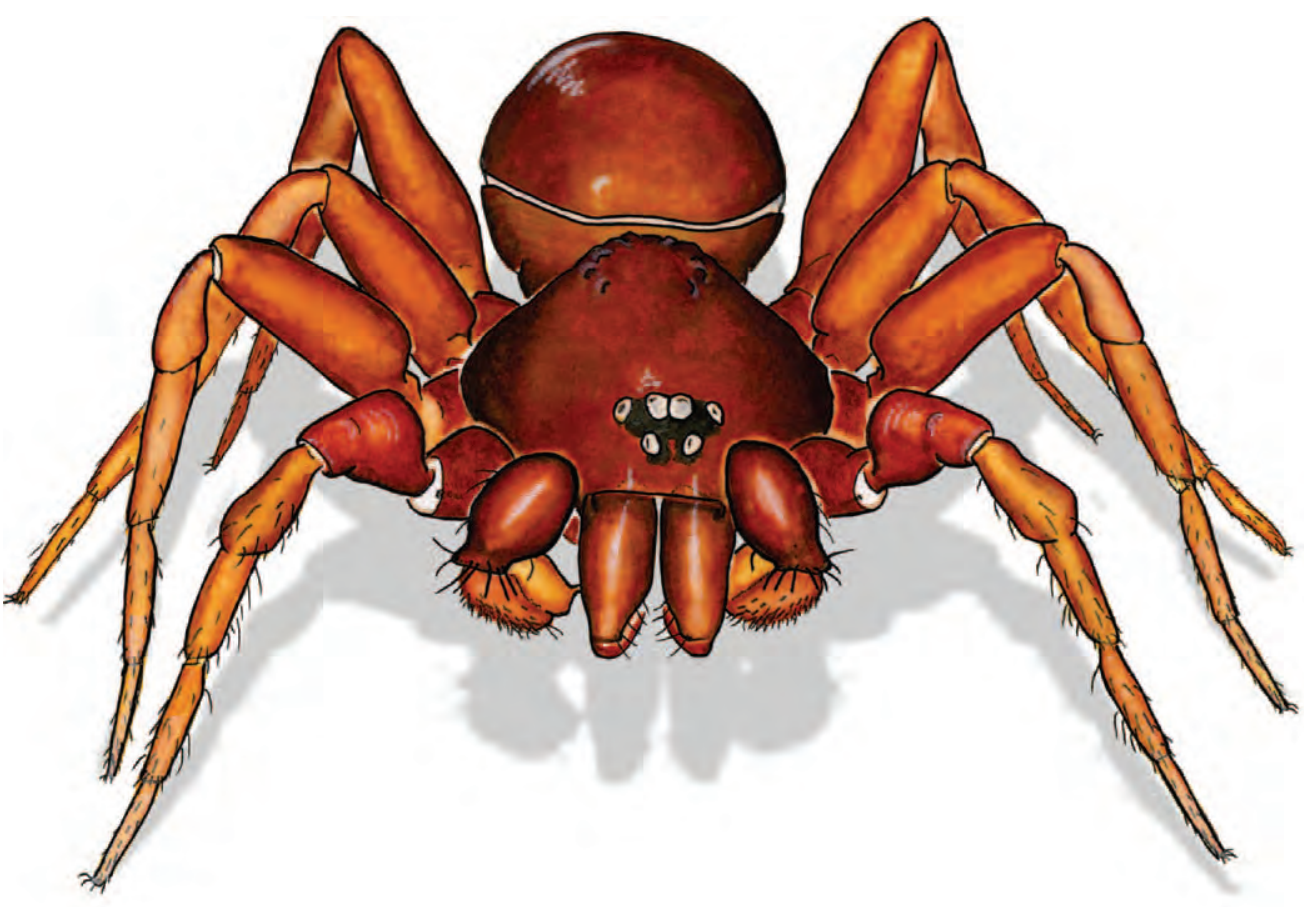

$\stackrel{\sim}{\sim}$

on the cover: Prethopalpus fosuma (Burger et al.) MALE (PBI_OON 12813), HABITUS, FRONTAL VIEW. WATERCOLOR BY WENDY HEBRON. 


\title{
THE NEW AUSTRALASIAN GOBLIN SPIDER GENUS PRETHOP ALPUS (ARANEAE, OONOPIDAE)
}

\author{
BARBARA C. BAEHR \\ Queensland Museum, P.O. Box 3300, \\ South Brisbane, Queensland 4101, Australia; \\ CSER, School of Environmental and Life Sciences, \\ University of Newcastle, Callaghan, \\ New South Wales 2308, Australia \\ MARK S. HARVEY \\ Department of Terrestrial Zoology \\ Western Australian Museum, Locked Bag 49, \\ Welshpool DC, Western Australia 6986, Australia; \\ Division of Invertebrate Zoology, \\ American Museum of Natural History \\ California Academy of Sciences, San Francisco; \\ School of Animal Biology \\ University of Western Australia, Crawley, \\ Western Australia 6009, Australia \\ MATTHIAS BURGER \\ Division of Invertebrate Zoology \\ American Museum of Natural History, Central Park \\ West at 79th Street, New York, New York 10024

\section{MARCO THOMA} \\ Natural History Museum Bern, Bernastrasse 15, \\ Bern CH-3005, Switzerland
}

BULLETIN OF THE AMERICAN MUSEUM OF NATURAL HISTORY

Number 369, 113 pp., 749 figures, 10 maps

Issued June 21, 2012 


\section{CONTENTS}

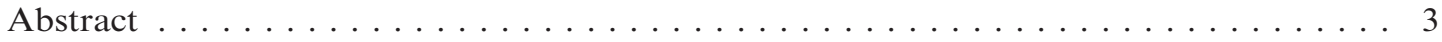

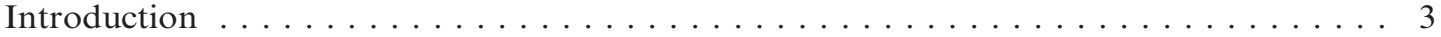

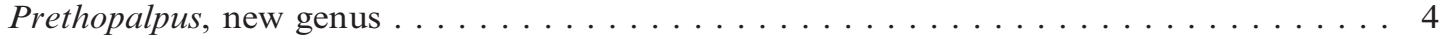

Species from Nepal and India . . . . . . . . . . . . . . . . . . . . 11

Species from Indonesia, Malaysia, Singapore, and Brunei . . . . . . . . . . . . . . 29

Species from Australia and Papua New Guinea . . . . . . . . . . . . . . . . . . . 78

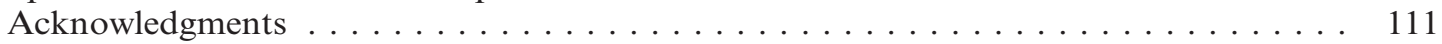

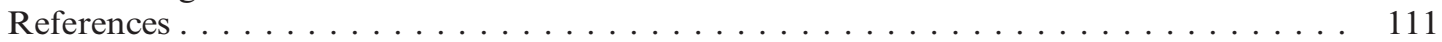

Index of Specific Names . . . . . . . . . . . . . . . . . . . . . . . . 113 


\begin{abstract}
The new goblin spider genus Prethopalpus is restricted to the Australasian tropics, from the lower Himalayan Mountains in Nepal and India to the Malaysian Peninsula, Indonesia, Papua New Guinea, and Australia. Prethopalpus contains those species with a swollen palpal patella, which is one to two times the size of the femur, together with a cymbium and bulb that is usually separated, although it is largely fused in four species. The type species Opopaea fosuma Burger et al. from Sumatra, and Camptoscaphiella infernalis Harvey and Edward from Western Australia are newly transferred to Prethopalpus. The genus consists of 41 species of which 39 are newly

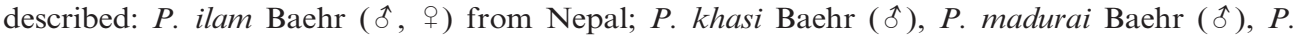

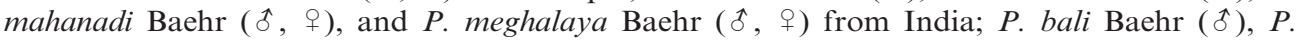

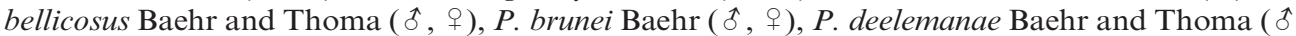

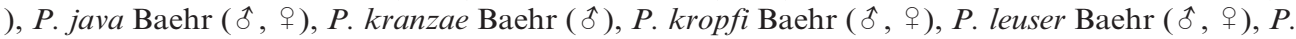

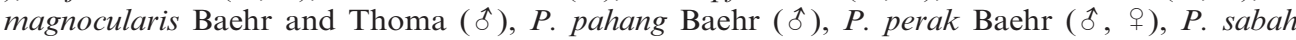

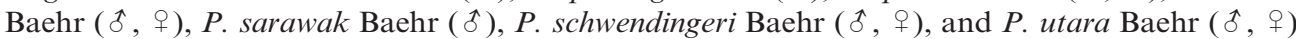
from Indonesia and Malaysia; and P. alexanderi Baehr and Harvey ( $\hat{\delta}), P$. attenboroughi Baehr

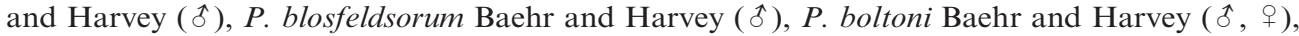

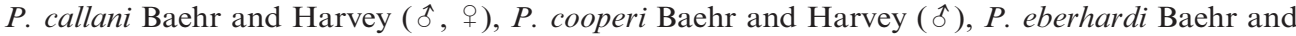
Harvey ( $\delta$, of), P. framenaui Baehr and Harvey ( $\delta$, + ) , P. humphreysi Baehr and Harvey $(\hat{\delta}, q)$,

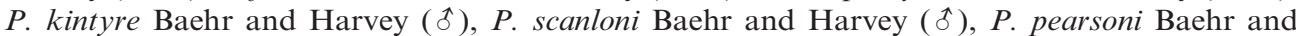

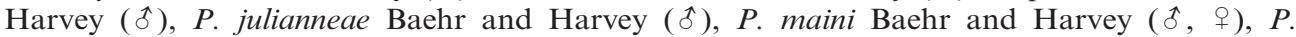

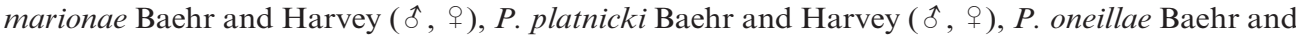
Harvey ( $\delta$ ), P. rawlinsoni Baehr and Harvey ( $\delta$ ), and P. tropicus Baehr and Harvey ( $\delta$, + ) from Australia and Papua New Guinea. Three separate keys to species from different geographical regions are provided. Most species are recorded from single locations and only three species are more widely distributed. A significant radiation of blind troglobites comprising 14 species living in subterranean ecosystems in Western Australia is discussed. These include several species that lack abdominal scuta, a feature previously used to define subfamilies of Oonopidae.
\end{abstract}

\section{INTRODUCTION}

Goblin spiders of the family Oonopidae are a diverse group with over 755 described species in 83 genera (Platnick, 2011), but the unnamed species may number at least 2,000 species (Baehr et al., 2010). Goblin spiders are generally small in size (adult body length of $0.5-4.0 \mathrm{~mm}$ ) and can be abundant in many terrestrial habitats including leaf litter, under bark of trees, in forest canopies, as inquilines, and in caves and other subterranean cavities (e.g., Wunderlich, 1994; Burger et al., 2002; Harvey and Edward, 2007b; Fannes and Jocqué, 2008; Platnick and Dupérré, 2009a, 2009 b; Baehr and Harvey, 2010; Baehr et al., 2010; Baehr and Ubick, 2010; Platnick and Dupérré, 2010b). They occur on all continents and the Australasian fauna is extensive with the number of unnamed species greatly outnumbering the meager named fauna.

The discovery of Opopaea fosuma Burger et al. from Sumatra provided the opportunity to present extensive illustrations and a comprehensive description of an Asian gob- lin spider (Burger et al., 2002). This species, however, exhibits differences from the typical morphology of species of Opopaea Simon (Platnick and Dupérré, 2009b), especially in the morphology of the male pedipalp, where the patella is about one to two times the size of the femur, connected to the femur subbasally or submedially, and the cymbium is not fused to the bulb. Species of Opopaea have the male palpal patella several times longer than the femur, is connected to the femur medially, and the cymbium and bulb are completely fused (e.g., Saaristo, 2001; Saaristo and Marusik, 2008; Platnick and Dupérré, 2009b). The blind Australian subterranean troglobite Camptoscaphiella infernalis Harvey and Edward shows similar genitalic morphology to $O$. fosuma, but was placed in Camptoscaphiella Caporiacco by Harvey and Edward (2007b) due to the lack of the features diagnostic of Opopaea. Since then, a review of Camptoscaphiella (Baehr and Ubick, 2010) has shown consistent differences between $C$. infernalis and all species now attributed to Camptoscaphiella, 
including the presence of spines on the anterior legs of Camptoscaphiella (Baehr and Ubick, 2010), and the lack of such spines in $C$. infernalis and O. fosuma (Burger et al., 2002; Harvey and Edward, 2007b).

The discovery of numerous new species from Australasia that are similar to $O$. fosuma and $C$. infernalis has allowed a better interpretation of their relationships. We here hypothesize that these species can be easily distinguished from Opopaea and Camptoscaphiella by the features discussed above, and we therefore propose a new genus, Prethopalpus, for these species. While the majority of species of this genus are rainforest litterdwelling species, a significant radiation of troglobitic species was detected in the arid regions of Western Australia.

\section{MATERIAL AND METHODS}

The specimens examined for this study are lodged in the following museums: American Museum of Natural History, New York (AMNH); Australian National Insect Collection, Canberra, Australia (ANIC); Field Museum of Natural History, Chicago (FMNH); Muséum d'Histoire Naturelle, Geneva, Switzerland (MHNG); Natural History Museum, Bern, Switzerland (NMB); Queensland Museum, Brisbane, Australia (QM); Senckenberg Museum, Frankfurt am Main, Germany (SMF); National Natuurhistorische Museum, Leiden, Netherlands (RMNH); University of Turku, Finland (UTU); and Western Australian Museum, Perth, Australia (WAM).

Specimens were examined using a Leica MZ16A microscope. Photomicrographic images were produced using a Leica DFC 500 and the software program AutoMontage Pro Version 5.2 (p). Specimens prepared for scanning electron microscopy were dehydrated in $100 \%$ ethanol, critical-point dried, sputter coated, and imaged with a Hitachi S4700 SEM. Descriptions were generated with the aid of the Planetary Biodiversity Inventory (PBI) descriptive goblin spider database and shortened where possible. Drawings were done of the left palp. All measurements are in millimeters. Abbreviations are used in the text as follows: ALE, anterior lateral eyes; ALS, anterior lateral spinnerets; EF, epigastric fold; GR, groove between tracheal spiracles; PL, median plate; PLE, posterior lateral eyes; PLS, posterior lateral spinnerets; PME, posterior median eyes; PMS, posterior median spinnerets. Scale bars for habitus and palpal images are $0.5 \mathrm{~mm}$, and epigynes are $0.1 \mathrm{~mm}$. Full-color, high-resolution versions of the images will be available on the goblin spider PBI website (http://research.amnh.org/oonopidae).

The species descriptions contain only the differences from the generic description. The description of the females includes just those differences from the male. To aid the reader, we treat species from different geographical separately, the first from India and Nepal, the second from Indonesia, Malaysia, Singapore, and Brunei, and the third from Australia and Papua New Guinea. Separate keys to species are provided for these regions.

Often older locality labels do not provide accurate geographical coordinates; latitudes and longitudes encased in parentheses indicate that the coordinates were obtained from the Google Earth website, and included in the locality information for mapping.

\section{SYSTEMATICS}

Family Oonopidae Simon, 1890 Prethopalpus new genus

Type SPecies: Opopaea fosuma Burger et al., 2002.

Diagnosis: Males of Prethopalpus resemble most species of Opopaea in having an enlarged palpal patella (e.g., figs. 62-130), but most species can be easily distinguished by the separate cymbium and bulb, which is fused in a few species (e.g., figs. 104-112); females and males lack a pair of small dorsolateral, triangular extensions on the pedicel as well as the paired curved scutal ridges on the scutopedicel region (e.g., fig. 537); females of Prethopalpus share with Opopaea the paddle-shaped sclerite and the naillike structure (e.g., figs. 134, 136), but have additionally a single, central receptaculum (e.g., figs. 58, 132, 134, 136) of various shapes.

ETYMOLOGY: The generic name refers to the swollen male pedipalpal patella (Greek pretho, "to swell, blow up, or inflate," and 


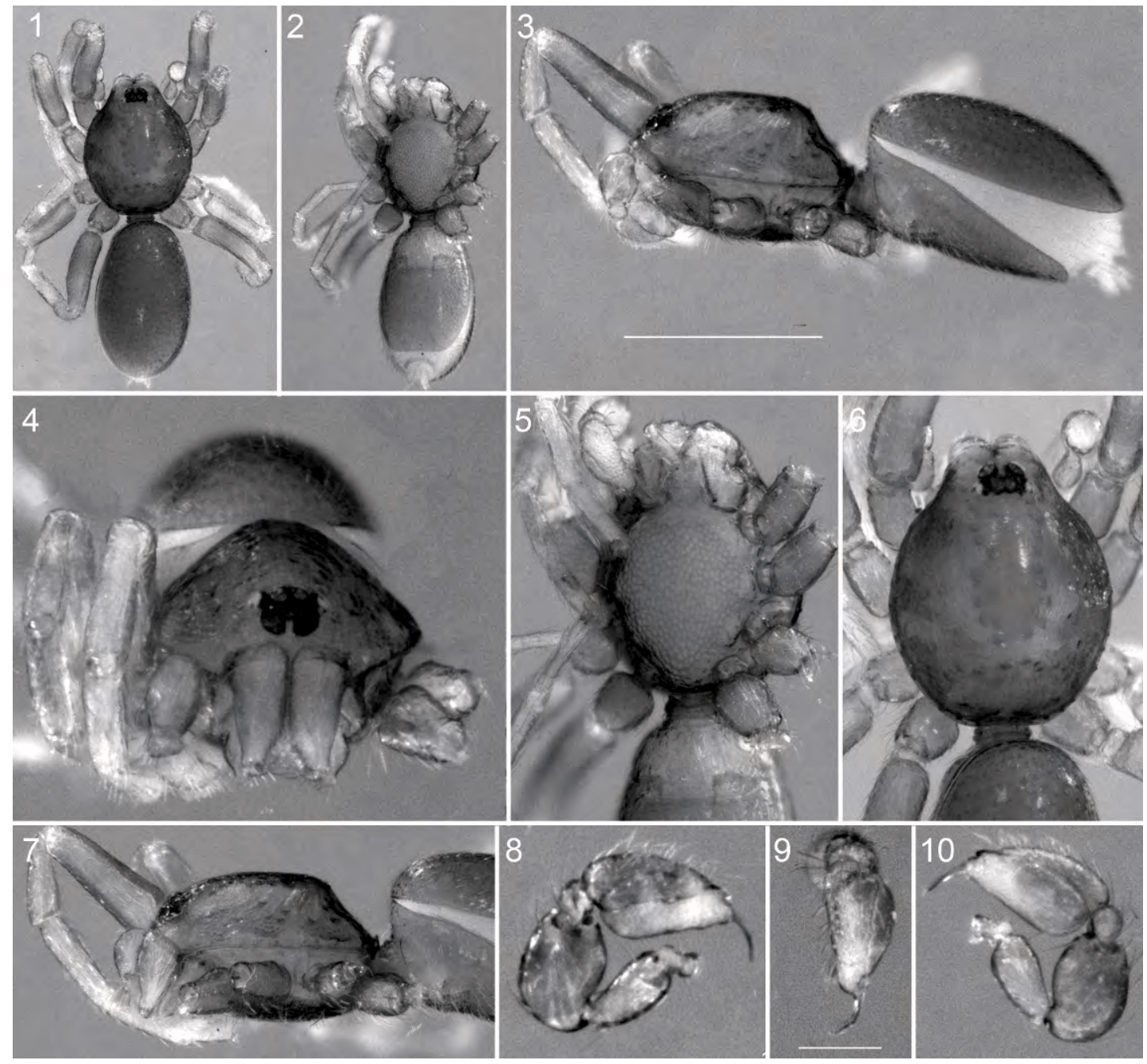

Figs. 1-10. Prethopalpus fosuma (Burger et al.), male (PBI_OON 16200). 1. Habitus, dorsal view. 2. Same, ventral view. 3. Same, lateral view. 4. Prosoma, anterior view. 5. Same, ventral view. 6. Same, dorsal view. 7. Same, lateral view. 8. Male palp, prolateral view 9. Same, dorsal view 10. Same, retrolateral view.

Latin palpus, "soft palm of the hand, feeler"). The gender is masculine.

DESCRIPTION: Total length of males 1.0 1.7, of females 1.2-1.8. Carapace pale orange to yellow-brown, without any pattern; ovoid in dorsal view (figs. 6, 16), pars cephalica flat or slightly elevated in lateral view (figs. 7, 17, $494,506,697)$, anteriorly narrowed to 0.49 times its maximum width or less, with rounded posterolateral corners, posterolateral edge without pits, posterior margin not bulging below posterior rim, anterolateral corners without extension or projections, posterolateral surface with (e.g., figs. 41, 42) or without small spikes, surface of elevated portion of pars cephalica and sides smooth, striated, or strongly reticulate, thorax without depressions, fovea absent, without radiating rows of pits; rebordered (fig. 19), with or without denticles; plumose setae near posterior margin of pars thoracica absent; nonmarginal pars cephalica setae light or dark, needlelike, present in U-shaped row (figs. 19, 41); nonmarginal pars thoracica setae absent (fig. 42); marginal setae light or dark, needlelike. Clypeus margin slightly 


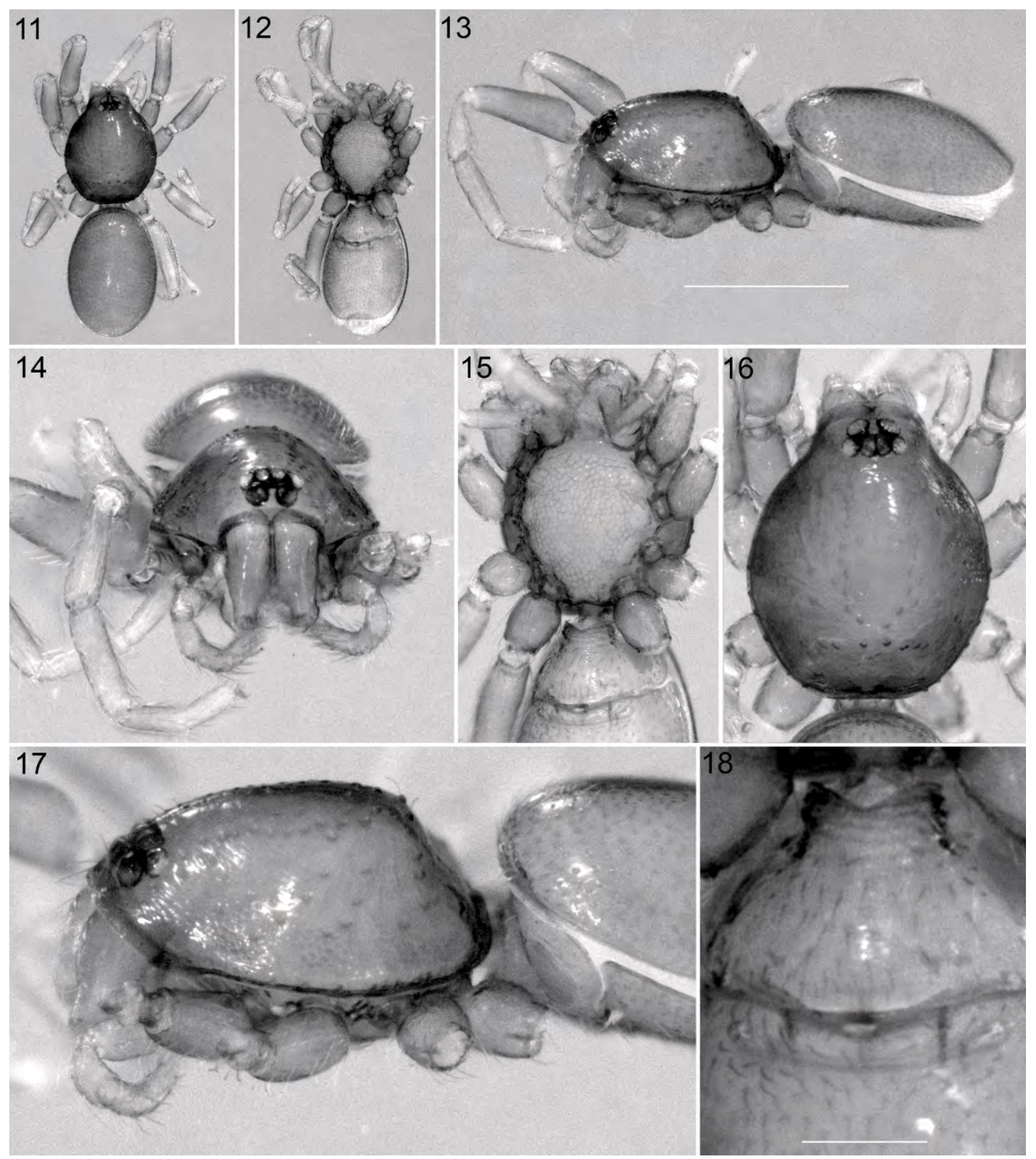

Figs. 11-18. Prethopalpus fosuma (Burger et al.), female (PBI_OON 12377). 11. Habitus, dorsal view. 12. Same, ventral view. 13. Same, lateral view. 14. Prosoma, anterior view. 15. Same, ventral view. 16. Same, dorsal view. 17. Same, lateral view. 18. Female epigyne, ventral view.

rebordered, curved downward in frontal view (e.g., fig. 4), sloping forward in lateral view (e.g., fig. 7), high, ALE separated from edge of carapace by their radius or more, median projection absent; setae present, light or dark, needlelike. Eyes: absent or with six eyes, well developed or reduced, subequal, ALE or PME largest (figs. 19, 357, 476), ALE circular, PME squared or circular, PLE circular; posterior eye row mostly recurved, sometimes straight or procurved from both above and front (figs. 357, 166, 195); ALE 


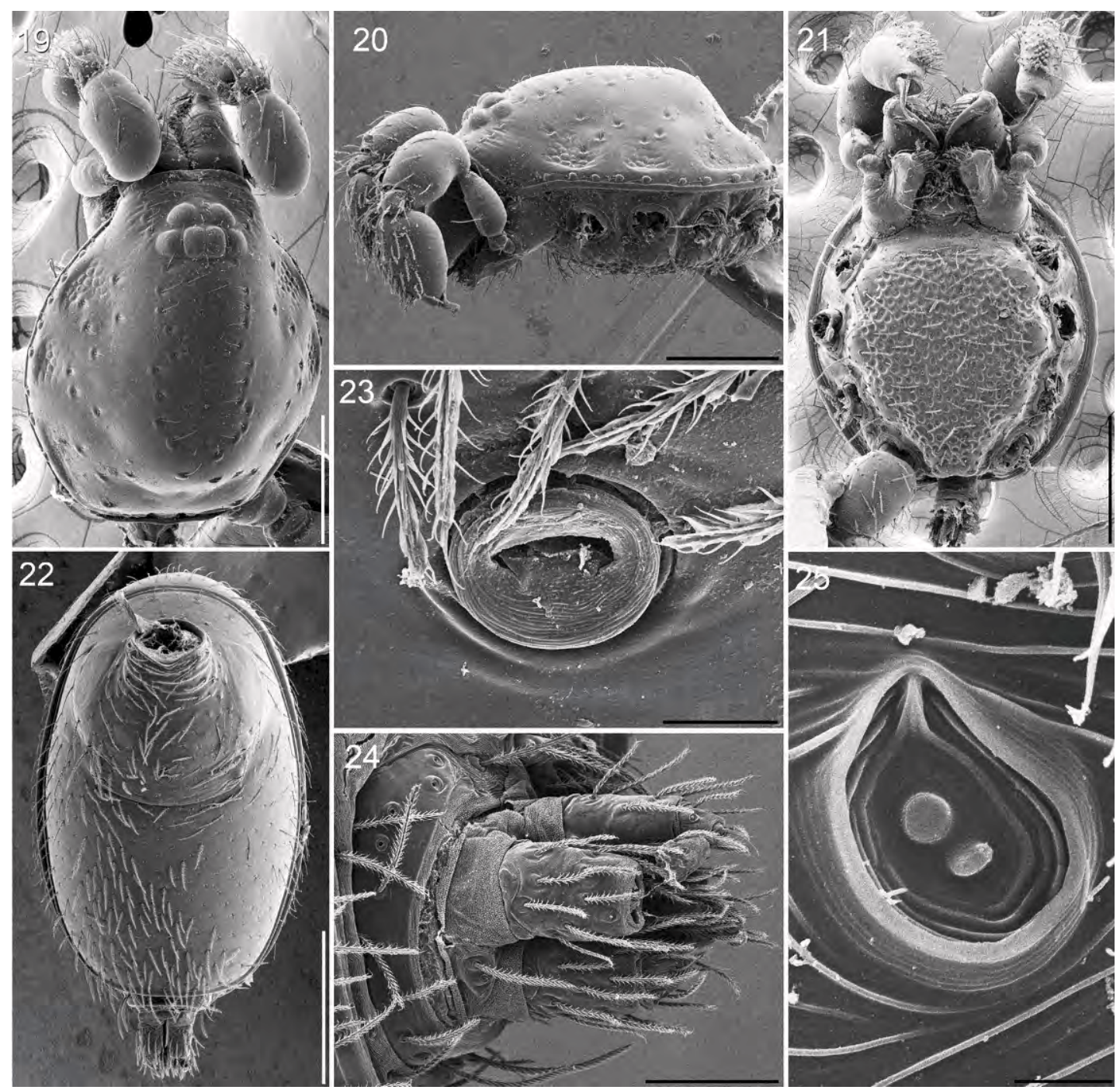

Figs. 19-25. Prethopalpus fosuma (Burger et al.), male, scanning electron micrographs. 19. Prosoma, dorsal view. 20. Same, lateral view. 21. Same, ventral view. 22. Abdomen, ventral view. 23 Sperm pore, ventral view. 24. Spinnerets, lateral view. 25. Tarsal organ of left leg III, dorsal view. Scale bars $=200 \mu \mathrm{m}$ (figs. 19-22), $50 \mu \mathrm{m}$ (fig. 24), $10 \mu \mathrm{m}$ (fig. 23), $2 \mu \mathrm{m}$ (fig. 25).

separated by less than their radius, ALE-PLE touching, PME touching for less than half their length, PLE-PME touching. Sternum longer than wide (e.g., figs. 21, 43), yellowish white or pale orange, uniform, fused or not fused to carapace, median concavity absent, without or with shallow radial furrows between coxae I-II, II-III, III-IV (e.g., fig. 250), surface smooth, finely reticulate, coarsely reticulate, with or without pits, sickleshaped structures absent, anterior margin unmodified (fig. 21), anterior corner unmodified, lateral margin with infracoxal grooves (fig. 21), distance between coxae approximately equal (fig. 21) or distance between coxae III and IV larger (fig. 695), with or without extensions of precoxal triangles (fig. 21), lateral margins unmodified, without posterior hump; posterior margin not extending posteriorly of coxae IV; setae 


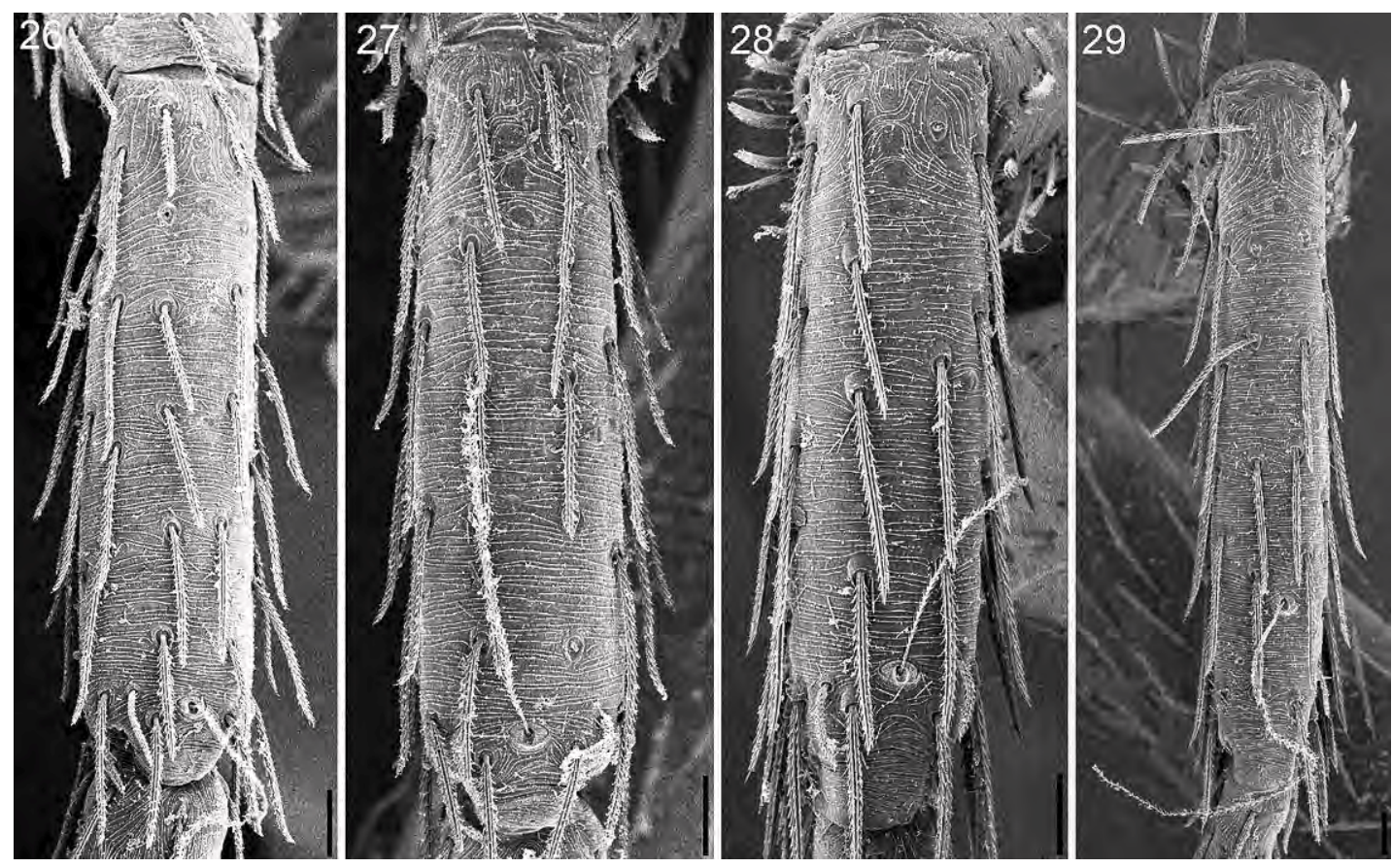

Figs. 26-29. Prethopalpus fosuma (Burger et al.), male metatarsi, dorsal view, scanning electron micrographs. 26. Left leg I. 27. Left leg II. 28. Left leg III. 29. Left leg IV. Scale bars $=20 \mu \mathrm{m}$.

sparse, light or dark, needlelike, evenly scattered, originating from small pits, without hair tufts. Mouthparts: chelicerae, endites, and labium pale orange. Chelicerae straight, anterior face unmodified; without teeth on promargin or retromargin; fang without toothlike projections, directed medially, shape normal, without prominent basal process, tip unmodified; setae needlelike, densest medially; paturon distal region unmodified, inner margin with scattered setae, posterior surface unmodified, promargin unmodified, inner margin unmodified, laminate groove absent or weakly developed. Labium (e.g., fig. 728) triangular, rarely trapezoidal or rectangular, fused or not to sternum, with two to five setae on anterior margin, subdistal portion with unmodified setae, anterior margin indented at middle, same as sternum in sclerotization. Endites distally not excavated, serrula present in single row (fig. 728), posteromedian part unmodified, anteromedian tip with small projection (fig. 728), same as sternum in sclerotization. Abdomen cylindrical or ovoid (figs. 22, 44, 488), without long posterior extension, rounded posteriorly, interscutal membrane rows of small sclerotized platelets absent posteriorly; dorsum soft portions white, without color pattern. Book lung covers, ovoid (figs. 22, 44), without setae, anterolateral edge unmodified. Posterior spiracles connected by groove. Pedicel tube short, rarely long, ribbed, or plain (e.g., figs. 22, 44, 482), scutopedicel region unmodified (fig. 22), scutum not extending far dorsal of pedicel (figs. 7, 17), plumose hairs absent, matted setae on anterior ventral abdomen in pedicel area absent, cuticular outgrowths near pedicel absent. Dorsal scutum sclerotized (e.g., fig. 3) or absent (e.g., fig. 556); when present, yellowbrown to pale orange, without color pattern, covering full length of abdomen, no soft tissue visible from above, not fused to epigastric scutum (fig. 22), middle surface smooth, sides smooth, anterior half without projecting denticles; epigastric scutum weakly sclerotized, surrounding pedicel, not protruding or protruding, small lateral sclerites absent (figs. 2, 12); postepigastric scutum weakly sclerotized, pale orange to yellow brown, long, semicircular, covering nearly full length of abdomen, fused to epigastric scutum in males, 


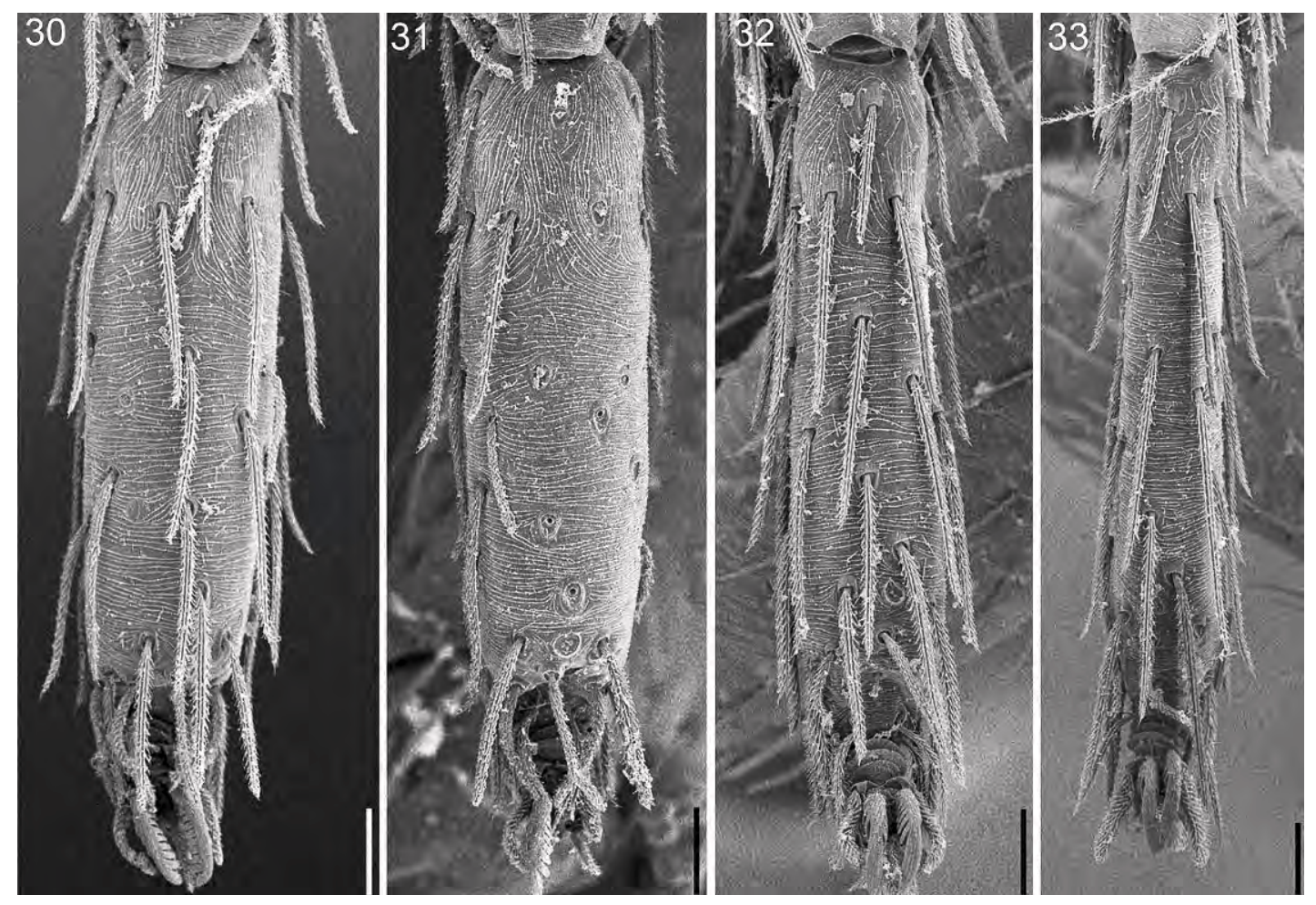

Figs. 30-33. Prethopalpus fosuma (Burger et al.), male tarsi, dorsal view, scanning electron micrographs. 30. Left leg I. 31. Left leg II. 32. Left leg III. 33. Left leg IV. Scale bars $=20 \mu \mathrm{m}$.

with short posteriorly directed lateral apodemes (figs. 5, 18); spinneret scutum present as incomplete ring, with fringe of stout setae. Dorsum, epigastric area, and postepigastric area setae present, light or dark, needlelike. Dense patch of setae anterior to spinnerets absent. Interscutal membrane with setae. Colulus represented only by two setae (fig. 44). Spinnerets in females (based on $P$. fosuma, figs. 45, 46): ALS (fig. 46) with four long spigots; PMS (fig. 45) with one long spigot; PLS (fig. 45) with two shorter spigots; spinnerets in males (fig. 24) not examined in detail. Legs: pale orange, yellow, or white, without color pattern; femur IV not thickened, same size as femora I-III, patella plus tibia I nearly as long as carapace, or shorter, tibia I unmodified, tibia IV specialized hairs on ventral apex absent, tibia IV ventral scopula absent, metatarsi I and II mesoapical comb absent, metatarsi III and IV weak ventral scopula absent, without leg spination. Superior claws examined in detail in $P$. fosuma (figs. 34-37); all surfaces striated; proclaws and retroclaws I-IV each with eight subapical teeth along inner margin; tarsi without inferior claw. Trichobothria: tibiae and metatarsi each with one distal trichobothrium (figs. 26-33, 48-51); base longitudinally narrowed, aperture internal texture not gratelike, hood covered by numerous low, closely spaced ridges. Tarsal organ of legs with two to three equal sized sensillae (figs. 31-33, 53, 54); tarsal organ of palpal tarsus with two sensillae (fig. 25). Male genitalia: epigastric region with small, circular or oval sperm pore situated at level of anterior spiracles (e.g., fig. 22), with or without strongly protruding extension (e.g., fig. 3), rebordered (fig. 23). Palp not strongly sclerotized, normal size, right and left palps symmetrical; trochanter normal size, unmodified; femur normal size, two or more times as long as trochanter, without posteriorly rounded lateral dilation; patella one to two times as long as femur, without prolateral row 


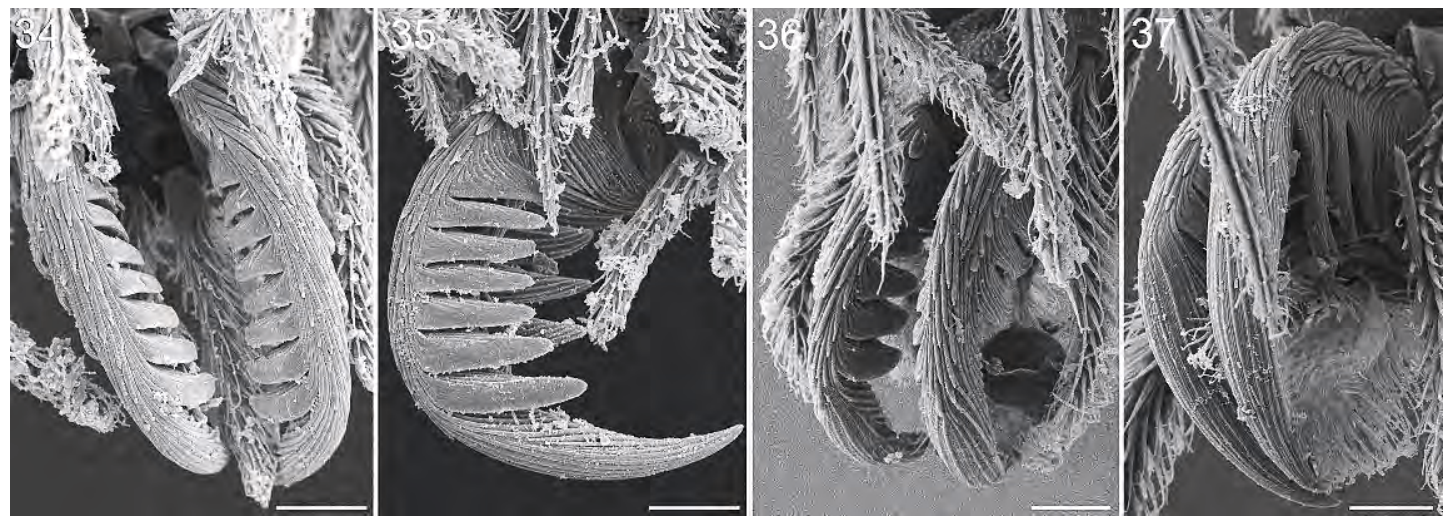

Figs. 34-37. Prethopalpus fosuma (Burger et al.), male claws, scanning electron micrographs. 34. Left leg I, dorsal view. 35. Left leg II, lateral view. 36. Left leg III, dorsolateral view. 37. Left leg IV, dorsolateral view. Scale bars $=5 \mu \mathrm{m}$.

of ridges, femur attaching to patella subbasally or submedially; setae unmodified; tibial trichobothria not examined; cymbium either not fused with bulb or nearly completely fused withbulb, but vestigial suture present; not extending beyond distal tip of bulb; bulb 1-1.5 times as long as cymbium, slender, tapering apically, plumose setae absent, without distal patch of setae, without stout setae. Embolus situated distally on bulb, usually long, bent medially, with or without conductor. Female genitalia (figs. 56-61): copulatory opening close to epigynal fold leading into single, central receptaculum variously shaped; small anterior sclerite variously shaped (e.g., figs. 134, 136); apodemes (fig. 57) originating close to epigynal fold, posteriorly directed, reaching beyond posterior groove.

BIOGEOGRAPHY: Species of Prethopalpus are found from the Himalayan Mountains through India and southeast Asia to New Guinea and northern Australia. The majority of species have been recorded from single locations, although some Asian and Australasian species are slightly more widespread (maps 1-10). Many species can be regarded as short-range endemics as defined by Harvey (2002) and Harvey et al. (2011).

Although the vast majority of species are ground-dwelling inhabitants of forest leaf litter, there is a remarkable radiation of 14 Australian troglobitic species showing full adaptations to a permanent subterranean lifestyle including the complete loss of eyes and pale coloration. In addition to these characters, some species ( $P$. humphreysi, $P$. framenaui, and $P$. boltoni) lack dorsal abdominal scuta. The presence or absence of abdominal scuta was used by Simon (1893) to define two separate groups of Oonopidae, the "Oonopidae loricatae" and "Oonopidae molles," respectively, which were later formalized into the subfamilies Gamasomorphinae and Oonopinae by Petrunkevitch (1923). The utility of this single feature has long been debatable, and Platnick and Dupérré (2010a) recently cast doubt on whether Gamasomorphinae can be regarded as a monophyletic taxon based on this single feature.

The presence of obligate subterranean species of Prethopalpus in Western Australia is unique, as no other troglobitic species of Prethopalpus have been found across the range of the genus, and no epigean species have been found in Australia west of eastern Queensland (maps 6-10). The presence of a suite of troglobitic species in the arid zone of Western Australia is consistent with patterns found in other arachnid taxa where the sole arid zone species are troglobites with the nearest epigean representatives found in rainforests to the north in the Kimberley region of Western Australia, or to the east in Queensland (e.g., Harvey, 1993, 2001; Harvey and Edward, 2007a; Harvey and Volschenk, 2007; Harvey et al., 2008; Harvey and Leng, 2008; Platnick, 2008). This pattern is consistent with the hypothesis that the 


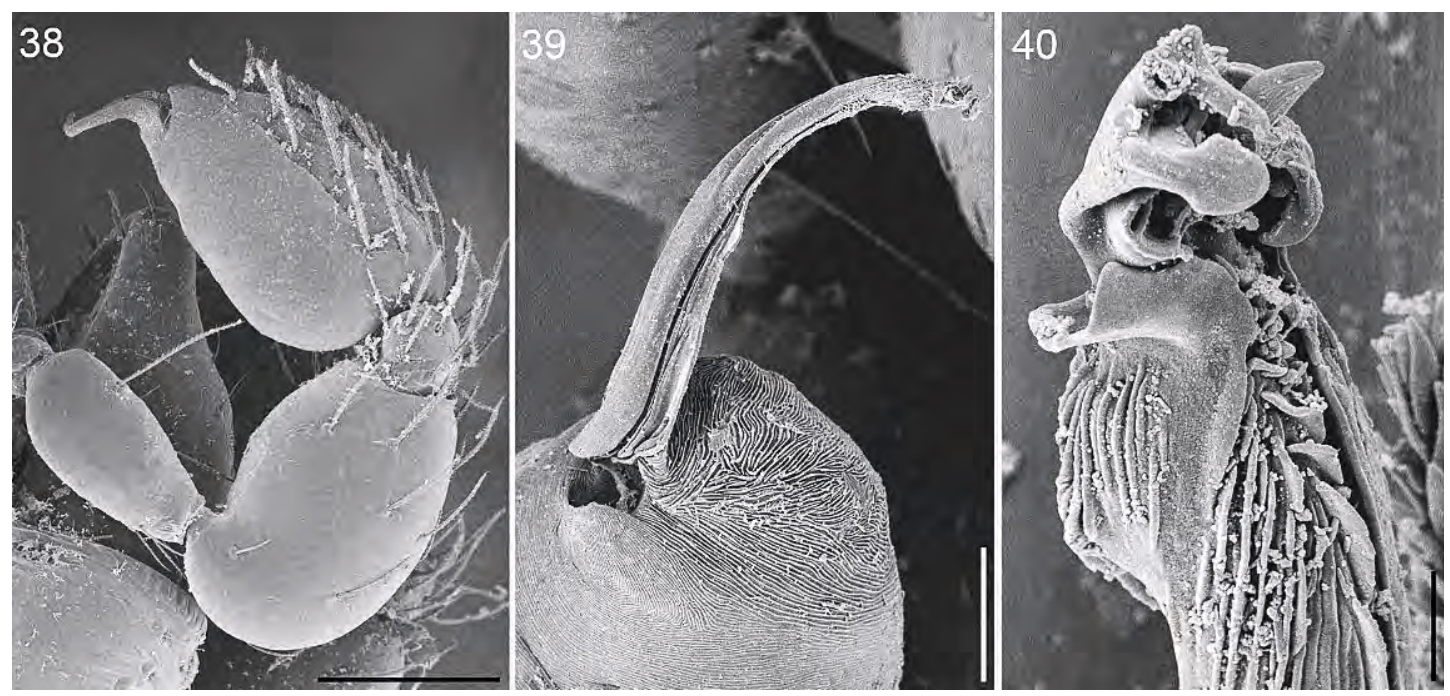

Figs. 38-40. Prethopalpus fosuma (Burger et al.), male palp, scanning electron micrographs. 38. Left palp, retrolateral view. 39. Left embolus, anterolateral view. 40. Tip of right embolus, retrolateral view. Scale bars $=100 \mu \mathrm{m}$ (fig. 38), $20 \mu \mathrm{m}$ (fig. 39), $2 \mu \mathrm{m}$ (fig. 40).

gradual drying of Australia since the Miocene resulted in the formation of vast arid regions (Byrne et al., 2008), forcing the original rainforest fauna to extinction or, in some rare cases, to form subterranean populations that eventually have acquired troglobitic adaptations (Guzik et al., 2011). We can find no morphological features to suggest that the subterranean Prethopalpus species form a monophyletic group, and it is likely that only molecular sequence data can test that hypothesis.

\section{SPECIES FROM NEPAL AND INDIA}

\section{Key to Species}

1. Males. . . . . . . . . . . . . . . . 2

- Females (unknown for $P$. khasi and $P$. madurai). . . . . . . . . . . . . 6

2. Embolar region with conductor (figs. 6267). . . . . . . . . . . . . 3

- Embolar region lacking conductor (figs. 68-

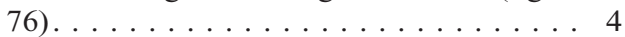

3. Conductor spatulate (fig. 64) .... . P. ilam

- Conductor sickleshaped (fig. 67). . . . . . . . . .

.............. P. mahanadi

4. Bulb ventrally bulging (figs. 68, 70, 197, 199); epigastric region strongly protruding (figs. 192, 196) . . . . . . . . . . . . P. madurai
Bulb not ventrally bulging (figs. 71, 74); epigastric region not protruding (figs. 163, 167) . . 5

5. Palpal femur about 1.4 times as long as patella, embolus slightly bent (figs. 74, 76, $168,170) \ldots \ldots \ldots \ldots \ldots$. . . khasi Palpal femur about as long as patella embolus strongly bent (figs. $71,73,226-228) \ldots \ldots \ldots \ldots$.

6. Copulatory opening broadly oval, with apical sclerotized hood; internal genital region with anterior sclerite rounded (figs. 135, 136). . . . . .

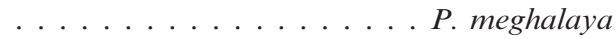
Copulatory opening rounded, without apical hood; internal genital region different . . 7

7. Internal genital region with anterior sclerite circular and drawn to a fine point (fig. 132)........... ............... P. ilam Internal genital region with anterior sclerite T-shaped (fig. 134) . . . . . . P. mahanadi

Prethopalpus khasi Baehr, new species Figures 74-76, 161-170; map 1

TYPE: INDIA: Meghalaya: Male holotype from Khasi Hills, au-dessus de Shillong, $25.56666^{\circ} \mathrm{N}, 91.88333^{\circ} \mathrm{E}, 1850 \mathrm{~m}$ (25 Oct. 1978, D. Burckhardt and I. Löbl), deposited in MHNG (PBI_OON 00023387).

ETYMOLOGY: The specific name is a noun in apposition taken from the type locality.

Diagnosis: Males resemble those of $P$. meghalaya, also from India, in general 


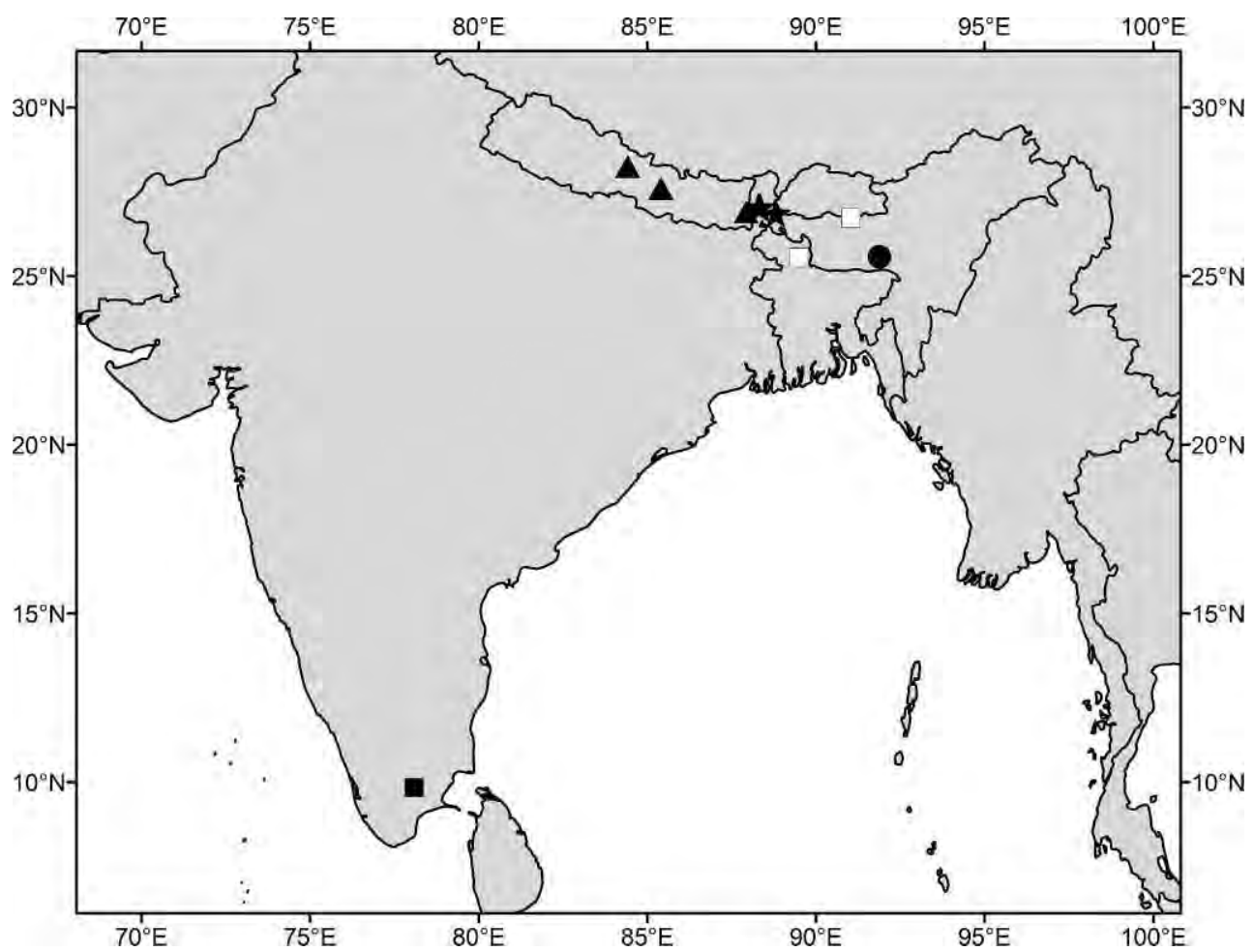

Map 1. Distribution records of Prethopalpus khasi (•), P. ilam (\), P. madurai (ם), P. mahanadi ( $\star$ ) and $P$. meghalaya $(\square)$.

habitus and eye sizes, but can be easily recognized by the much longer palpal femur, which is 1.4 times as long as the patella (figs. 74, 76).

MALE (PBI_OON 23387, figs. 161-170): Total length 1.22. Prosoma, mouthparts, and abdominal scuta pale orange, surface of elevated portion of pars cephalica smooth, sides finely reticulate; lateral margin without denticles. Eyes: six, tiny; ALE 0.037; PME 0.024; PLE 0.023; ALE largest, all eyes circular; posterior eye row straight from both above and front; ALE separated by their radius to diameter, ALE-PLE separated by less than ALE radius, PME separated by less than their radius, PLE-PME separated by less than PME radius. Sternum longer than wide, surface smooth, without pits, microsculpture absent, extensions of precoxal triangles absent. Endites twice as long as wide, with tiny toothlike projection. Abdomen ovoid; book lung covers large; pedicel tube short, ribbed. Dorsal scutum covering full length of abdomen, middle surface smooth, sides smooth; epigastric scutum not protruding; postepigastric scutum, almost rectangular, covering nearly full length of abdomen. Legs yellow; patella plus tibia I nearly as long as carapace. Sperm pore oval. Palp yellow; embolus light; femur about 1.4 times as long as patella, connected subbasally, patella slightly swollen, bulb well separated from cymbium, pyriform, with thin, medially bent embolus (figs. 74-76).

Female: Unknown.

OTHER MATERIAL EXAMINED: INDIA: Meghalaya: Khasi Hills, au-dessus de Shillong, $25.56666^{\circ} \mathrm{N}, 91.88333^{\circ} \mathrm{E}, 1850 \mathrm{~m}, 25$ Oct. 1978, D. Burckhardt and I. Löbl, 1 ๙ (MHNG, PBI_OON 00023387).

DISTRIBUTION: Prethopalpus khasi is known only from the Khasi Hills in northern India (map 1).

Prethopalpus ilam Baehr, new species Figures 62-64, 131, 132, 171-189; map 1

TYPES: NEPAL: Ilam District: Male holotype from $5 \mathrm{~km} \mathrm{~N}$ of Sanishare, foot of Siwalik 


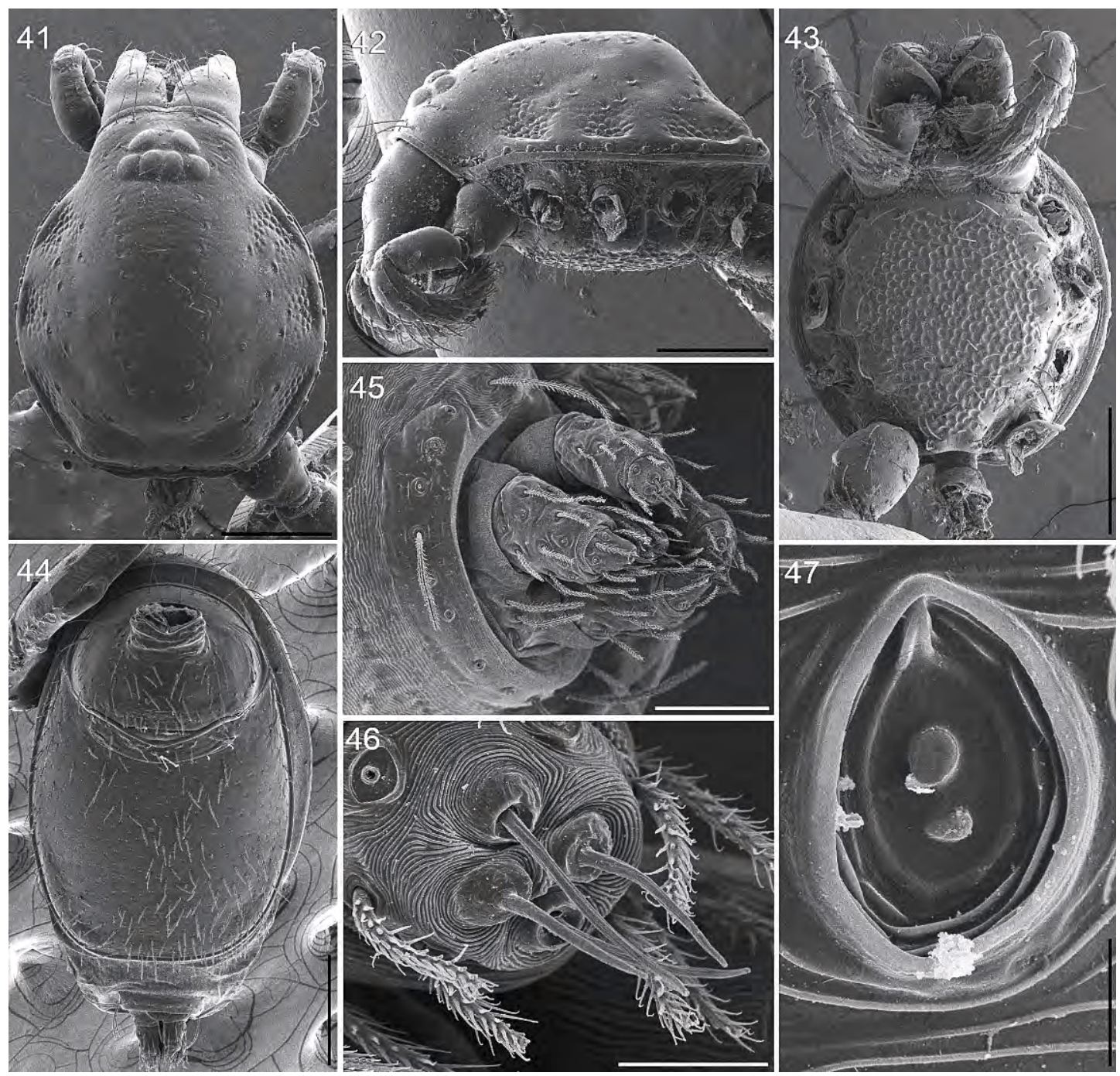

Figs. 41-47. Prethopalpus fosuma (Burger et al.), female, scanning electron micrographs. 41. Prosoma dorsal view. 42. Same, lateral view. 43. Same ventral view. 44. Abdomen, ventral view. 45. Spinnerets, posterolateral view. 46. Spigots, posterolateral view. 47. Tarsal organ of left leg III, dorsal view. Scale bars $=200 \mu \mathrm{m}$ (figs. 41-47), $50 \mu \mathrm{m}$ (fig. 45), $10 \mu \mathrm{m}$ (fig. 46), $2 \mu \mathrm{m}$ (fig. 47).

Mountains $\left[26.90000^{\circ} \mathrm{N}, 87.93000^{\circ} \mathrm{E}\right], 270 \mathrm{~m}$ (3-5 Apr. 1988, Martens and Schawaller), deposited in SMF (PBI_OON 00023390). Female allotype collected with holotype, deposited in SMF (PBI_OON 00023391).

ETYMOLOGY: The specific name is a noun in apposition taken from the type locality.

DiAgNOSIS: This species resembles those of $P$. mahanadi from India in body shape and eye size; males have a conductor at the tip of the bulb but can be easily recognized by the spatulate shape of the conductor (figs. 6264). Females can be separated by the long and square internal genital structure, with the anterior sclerite circular and drawn to a fine point (fig. 132).

Male (PBI_OON 15765, figs. 171-180): Total length 1.30. Carapace, mouthparts, and abdominal scuta pale orange, surface of elevated portion of pars cephalica smooth, sides smooth; lateral margin without denticles. Eyes: ALE 0.058; PME 0.049; PLE 


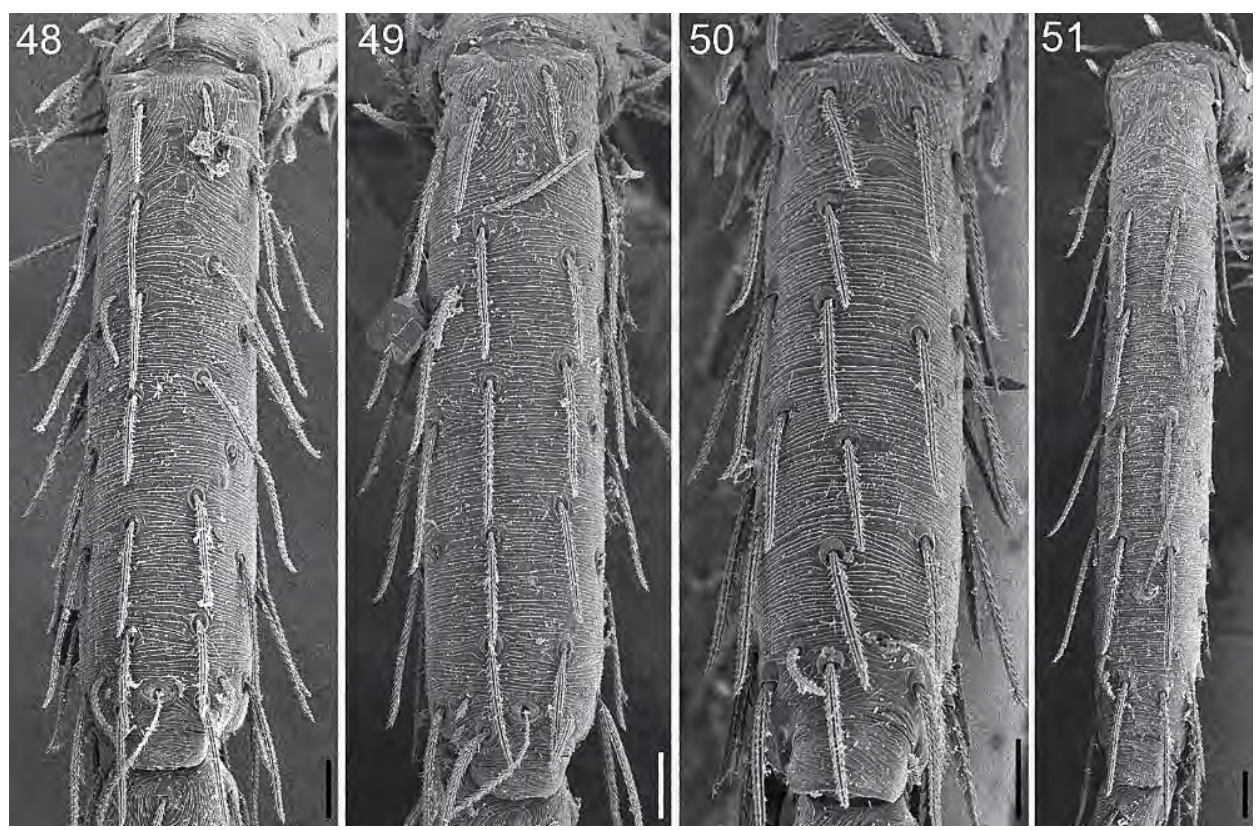

Figs. 48-51. Prethopalpus fosuma (Burger et al.), female metatarsi, dorsal view, scanning electron micrographs. 48. Left leg I. 49. Left leg II. 50. Left leg III. 51. Left leg IV. Scale bars $=20 \mu \mathrm{m}$.

0.040; ALE largest, ALE circular, PME squared, PLE circular; posterior eye row procurved from above, straight from front; ALE separated by their radius to diameter, ALE-PLE separated by less than ALE radius, PME touching throughout most of their length, PLE-PME touching. Sternum longer than wide, surface smooth, covered with small round pits, extensions of precoxal triangles absent. Endites about 1.5 times as long as wide, with small blunt toothlike projection. Abdomen ovoid; book lung covers large; pedicel tube short, ribbed. Dorsal scutum covering full length of abdomen, middle surface smooth, sides smooth; epigastric scutum not protruding; postepigastric scutum long, almost rectangular, covering nearly full length of abdomen. Legs yellow; patella plus tibia I shorter than carapace. Palp yellow; embolus dark, distal tip with large spatulate conductor and big retrodorsal concavity; femur attaching to patella subbasally; patella about as long as femur; cymbium not fused with bulb, bulb distal tip with large spatulate conductor and big retrodorsal concavity (figs. 62-64).
Female (PBI_OON 23391, figs. 181189): Total length 1.49. Eyes: ALE 0.049; PME 0.041; PLE 0.033. Epigastric area with oval epigynal opening, internal genital structure long and square, with anterior dropshaped sclerite (figs. 131, 132).

Other SPecimens ExAmined: NEPAL: Bagmati: Godawari, $27.60000^{\circ} \mathrm{N}, 85.40000^{\circ} \mathrm{E}$, 1600 m, 31 Mar. 1984, I. Löbl, A. Smetana, 1 우 (MHNG, PBI_OON 00015515); Ilam District, between Mai Pokhari and Ilam $\left[26.90000^{\circ} \mathrm{N}\right.$, $\left.87.93000^{\circ} \mathrm{E}\right], 1330 \mathrm{~m}, 1$ Apr. 1980, Martens and Ausobsky, 5 ㅇ (SMF, PBI_OON 00015790); Ilam District, Sanishare, $5 \mathrm{~km} \mathrm{~N}$, base of Siwalik Mountains [26.90000 $\left.\mathrm{N}, 87.93000^{\circ} \mathrm{E}\right]$, 270 m, 3-5 Apr. 1988, Martens and Schawaller, 1 s (SMF, PBI_OON 00015765); Lamjung District, Marsyandi, Senghe-Jagat $\left[28.25000^{\circ} \mathrm{N}\right.$, $\left.84.41667^{\circ} \mathrm{E}\right], 1100 \mathrm{~m}, 11$ Apr. 1980, Martens and Ausobsky, 1 (SMF, PBI_OON 00015789).

DisTRIBUTION: This species is known only from Nepal (map 1).

Prethopalpus madurai Baehr, new species Figures 68-70, 190-199; map 1

TYPE: Male holotype from INDIA: Tamil Nadu: $21 \mathrm{~km} \mathrm{~N}$ of Madurai, $9.83333^{\circ} \mathrm{N}$, 


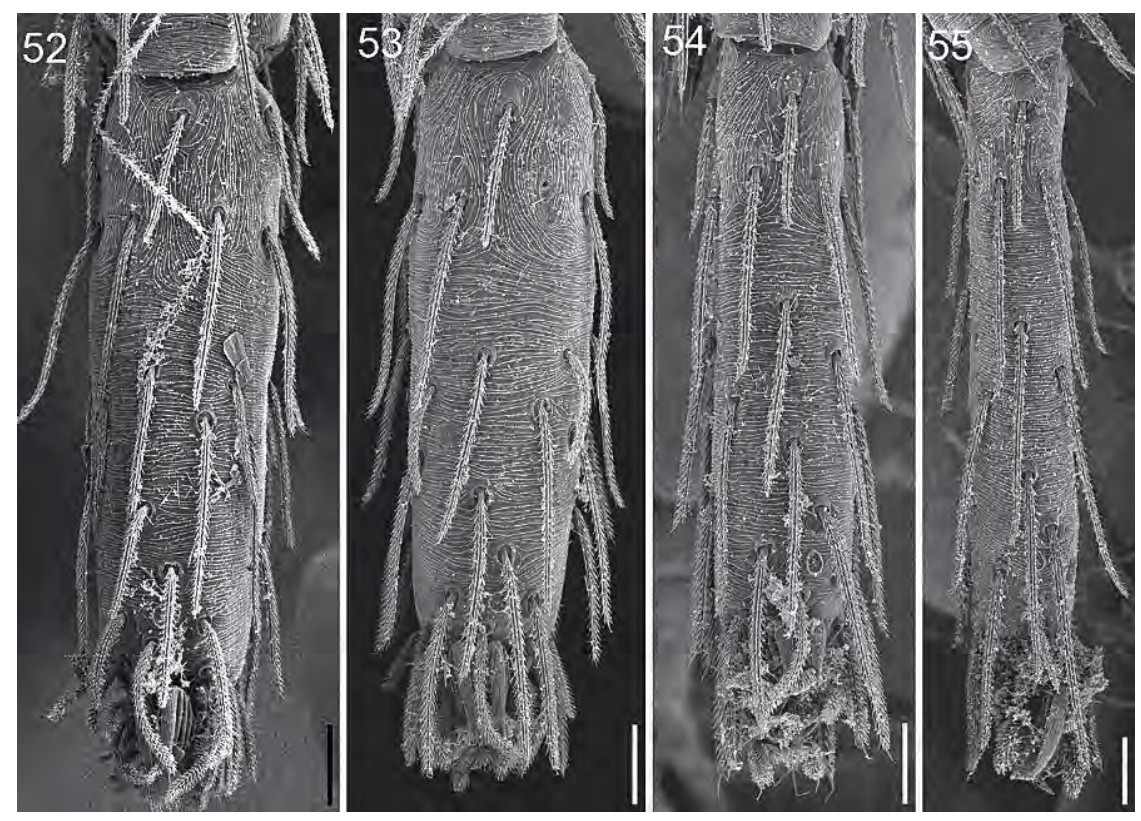

Figs. 52-55. Prethopalpus fosuma, female tarsi, dorsal view, scanning electron micrographs. 52. Left leg I. 53. Left leg II. 54. Left leg III. 55. Left leg IV. Scale bars $=20 \mu \mathrm{m}$.

$78.08333^{\circ} \mathrm{E}, 250 \mathrm{~m}(2$ Nov. 1978, C. Besuchet and I. Löbl), deposited in MHNG (PBI_OON 00015598).

ETYMOLOGY: The specific name is a noun in apposition taken from the type locality.

DiAGNOSIS: Males resemble those of $P$. attenboroughi from Australia in body shape, eye size, and palpal shape, but can be easily separated by the more spherical, ventrally bulging bulb (figs. 68, 70).

MALE (PBI_OON 15598, figs. 190-199): Total length 1.27. Prosoma, mouthparts, and abdominal scuta pale orange, finely reticulate; lateral margin without denticles. Eyes: six, small, ALE 0.036; PME 0.032; PLE 0.034; ALE largest, all eyes circular; posterior eye row procurved from both above and front; ALE separated by their radius to diameter, ALE-PLE separated by less than ALE radius, PME separated by less than their radius, PLE-PME separated by less than PME radius. Sternum longer than wide, surface coarsely reticulate. Endites twice as long as wide, with tiny toothlike projection. Abdomen ovoid; book lung covers large; pedicel tube short, ribbed. Dorsal scutum covering more than $3 / 4$ of abdomen, smooth; epigastric scutum strongly protruding; post- epigastric scutum long, semicircular, covering about $3 / 4$ of abdomen, without posteriorly directed lateral apodemes. Legs yellow; patella plus tibia I nearly as long as carapace. Epigastric region with oval sperm pore and strongly protruding extension. Palp yellow; embolus light; femur attaching to patella subbasally; patella longer than femur; cymbium not fused with bulb, bulb ventrally bulging, embolus ending in a blunt tip (figs. 68-70).

FEMALE: Unknown.

Other Material Examined: None.

Distribution: Prethopalpus madurai is known only from Madurai in India (map 1).

Prethopalpus mahanadi Baehr, new species Figures 65-67, 133, 134, 200-218; map 1

TyPes: INDIA: West Bengal: Male holotype from District Darjeeling, Mahanadi près de Kurseong [ $\left.26.8550^{\circ} \mathrm{N}, 88.8120^{\circ} \mathrm{E}\right], 1200 \mathrm{~m}$, 6 Oct. 1978 (C. Besuchet and I. Löbl), deposited in MHNG (PBI_OON 00015408). Female allotype collected with holotype, deposited in MHNG (PBI_OON 00012700).

ETYMOLOGY: The specific name is a noun in apposition taken from the type locality. 


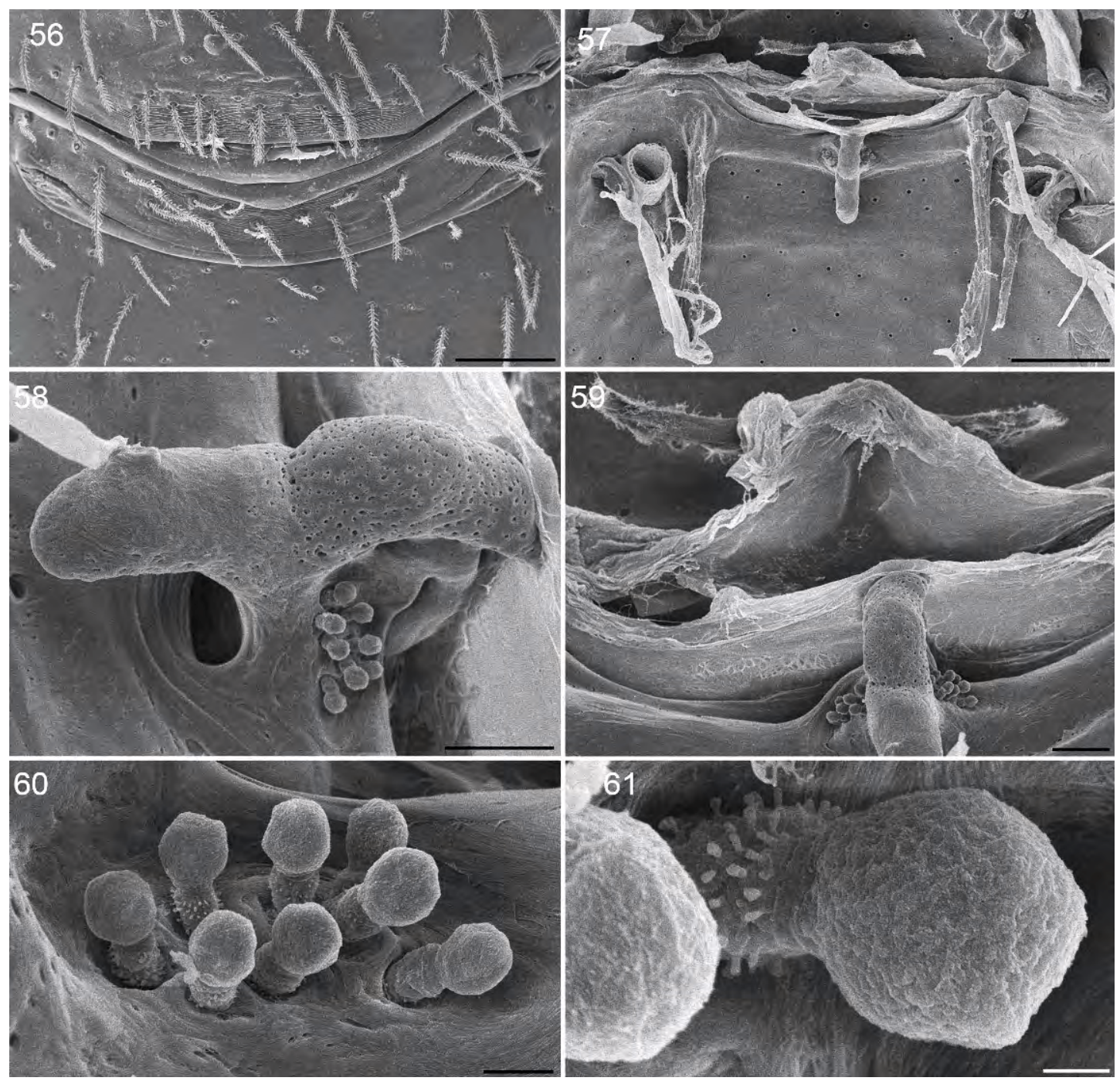

Figs. 56-61. Prethopalpus fosuma (Burger et al.), female genital area and internal genitalia, scanning electron micrographs. 56. Genital area, ventral view. 57. Internal genitalia, dorsal view. 58. Receptaculum, lateral view. 59. Uterus externus and T-shaped sclerite, posterior view. 60. Gland ducts around receptaculum, lateral view. 61. Close up of gland ducts around receptaculum. Scale bars $=50 \mu \mathrm{m}$ (figs. 56, 57), $10 \mu \mathrm{m}$ (figs. 58, 59), $2 \mu \mathrm{m}$ (fig. 60), $0.5 \mu \mathrm{m}$ (fig. 61).

Diagnosis: The species resembles P. ilam, also from India, in body shape and eye size; males in having a conductor at the tip of the bulb, but can be easily recognized by the sickleshaped conductor (figs. 65-67). Females can be separated by the internal genital structure with $\mathrm{T}$-shaped anterior sclerite (fig. 134).

Male (PBI_OON 15408, figs. 200-209): Total length $1 . \overline{5} 4$. Prosoma, mouthparts, and abdominal scuta pale orange, carapace surface smooth, sides slightly striated; lateral margin without denticles. Eyes: ALE 0.047; PME 0.042; PLE 0.038; ALE largest, ALE circular, PME oval, PLE circular; posterior eye row recurved from above, straight from front; ALE separated by their radius, ALEPLE separated by less than ALE radius, PME touching for less than half their length, PLE-PME separated by less than PME 

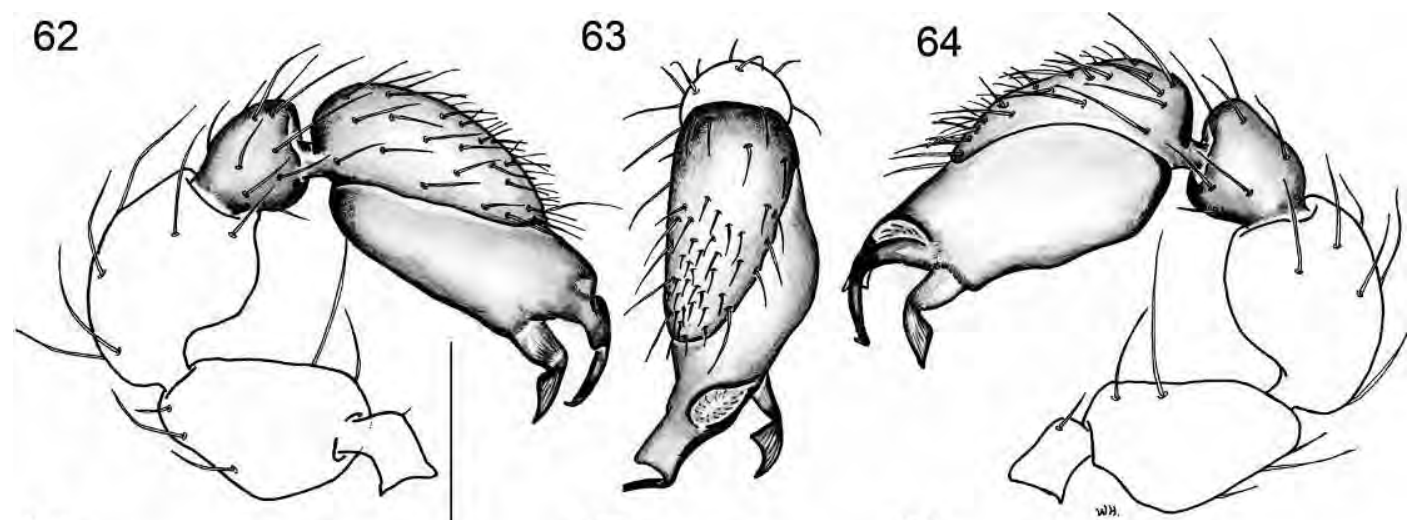

65
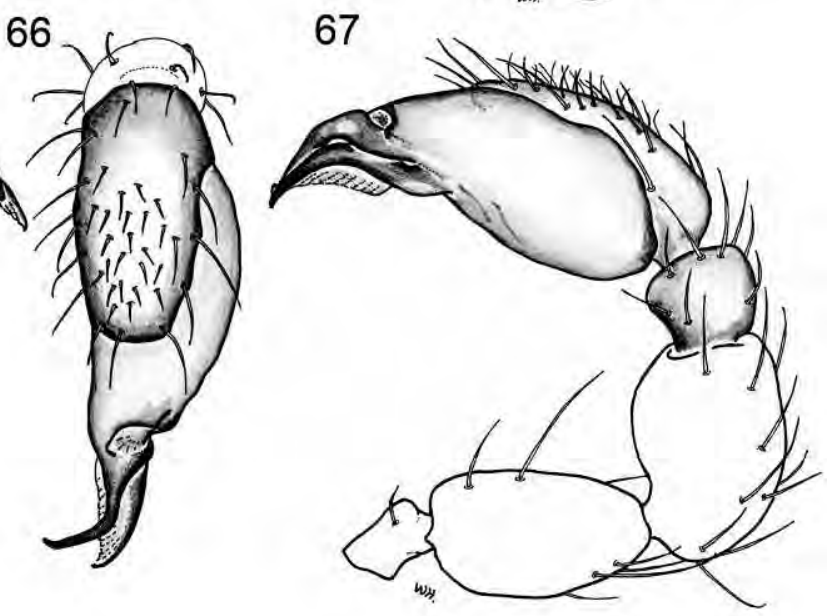

68

69
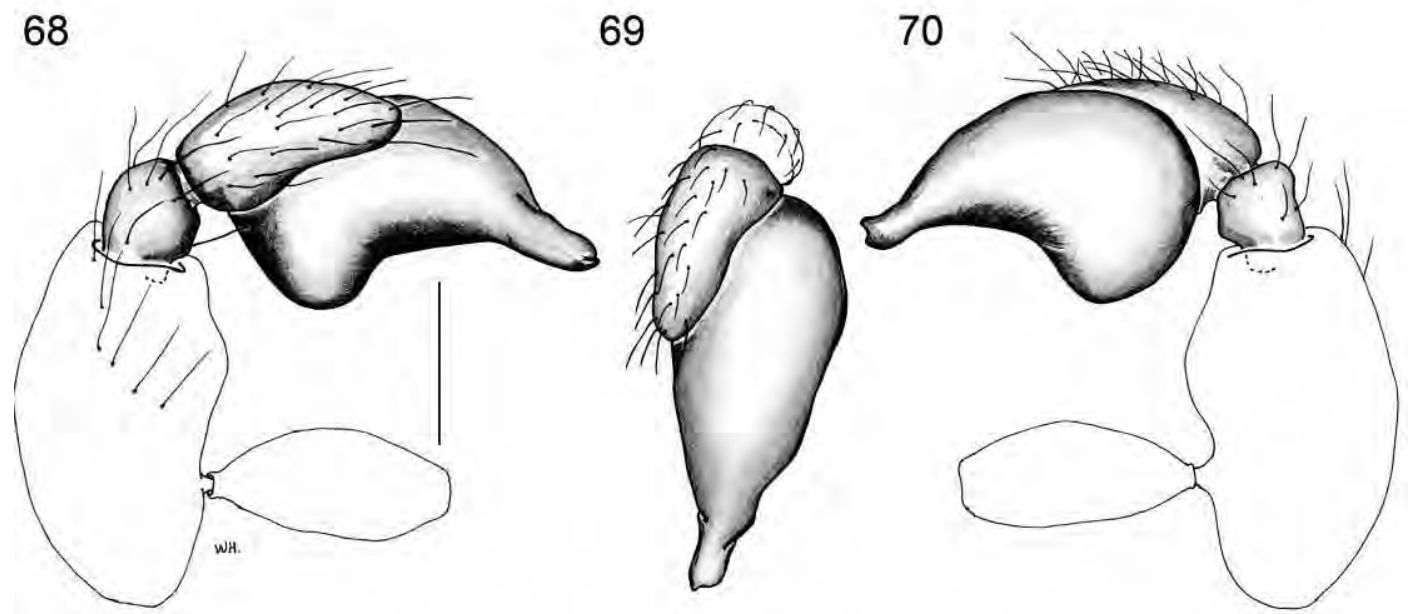

Figs. 62-70. Left palps, male: Prethopalpus ilam, n. sp. (PBI_OON 23390), Prethopalpus mahanadi, n. sp. (PBI_OON 15408), Prethopalpus madurai, n. sp. (PBI_OON 15598). 62. P. ilam, prolateral view. 63. Same, dorsal view. 64. Same, retrolateral view. 65. P. mahanadi, prolateral view. 66. Same, dorsal view. 67. Same, retrolateral view. 68. P. madurai, prolateral view. 69. Same, dorsal view. 70. Same, retrolateral view. Scale bars $=0.1 \mathrm{~mm}$. 

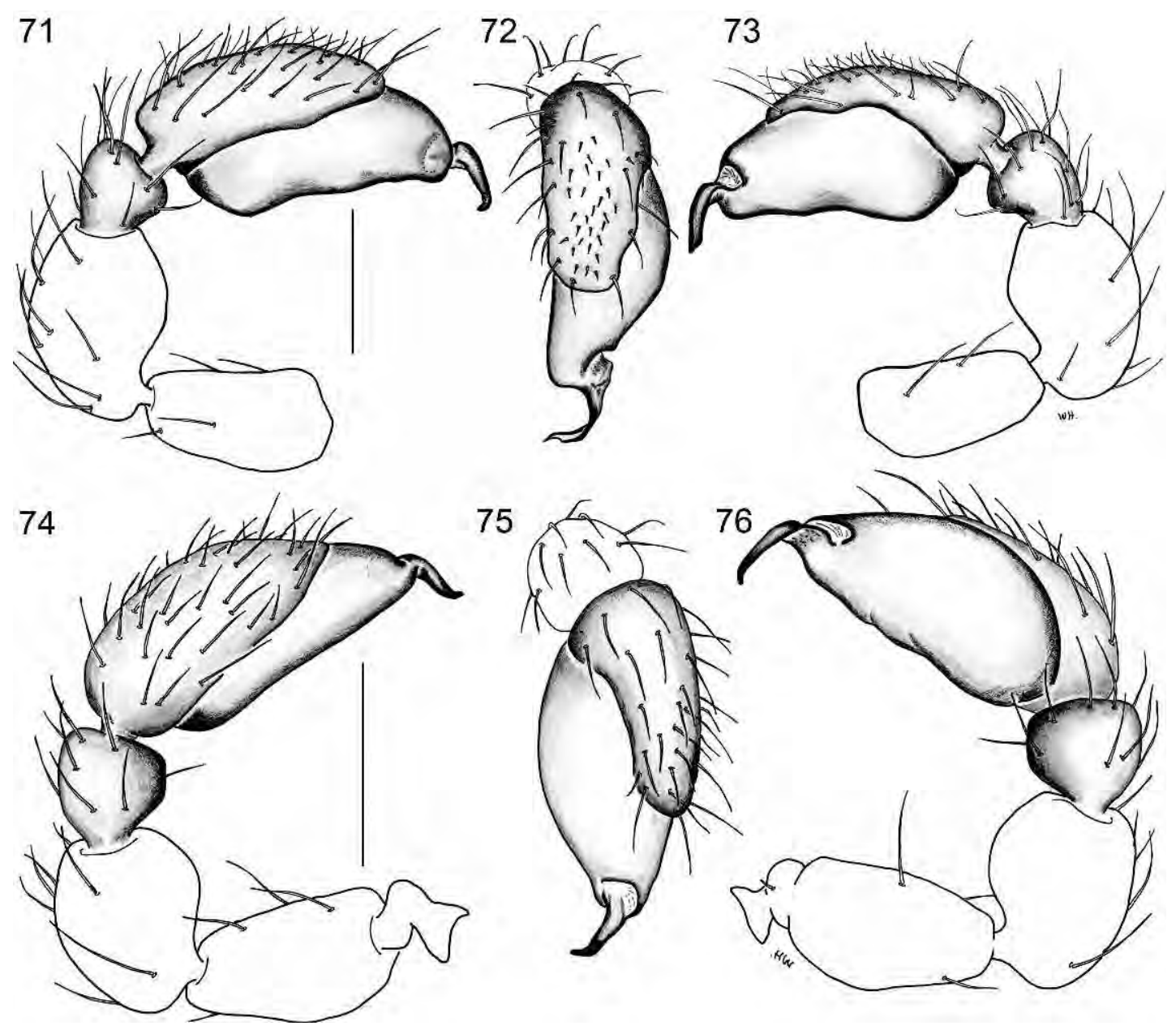

Figs. 71-76. Left palps, male: Prethopalpus meghalaya, n. sp. (PBI_OON 15410), Prethopalpus khasi, n. sp. (PBI_OON 23387). 71. P. meghalaya, prolateral view. 72. Same, dorsal view. 73. Same, retrolateral view. 74. . khasi, prolateral view. 75. Same, dorsal view. 76. Same, retrolateral view.

radius. Sternum longer than wide, surface smooth, covered with small round pits. Endites about twice as long as wide; toothlike projection distally rounded. Abdomen ovoid; book lung covers large; pedicel tube short, ribbed. Dorsal scutum covering full length of abdomen smooth; epigastric scutum not protruding; postepigastric scutum almost rectangular, covering nearly full length of abdomen. Legs: yellow; patella plus tibia I shorter than carapace. Epigastric region with sperm pore oval. Palp yellow; embolus dark; femur attaching to patella subbasally; patella about as long as femur, slightly larger than femur; cymbium not fused with bulb, bulb pyriform with long, sickleshaped conductor and medially bent embolus (figs. 65-67).

FeMAle (PBI_OON 12700, figs. 210218): Total length 1.56. Eyes: ALE 0.055; PME 0.047; PLE 0.047. Internal genital structure oval, with $\mathrm{T}$-shaped anterior sclerite (figs. 133, 134).

OTHER MATERIAL EXAMINED: INDIA: West Bengal: Darjeeling: Singla, $300 \mathrm{~m}$, tamisage dans une fôret, $27.5000^{\circ} \mathrm{N}$, $88.3000^{\circ} \mathrm{E}, 300 \mathrm{~m}, 17$ Oct. 1978 , C. Besuchet and I. Löbl, 1 q (MHNG, PBI_OON 00012874); entre Kalimpong et Algarah 

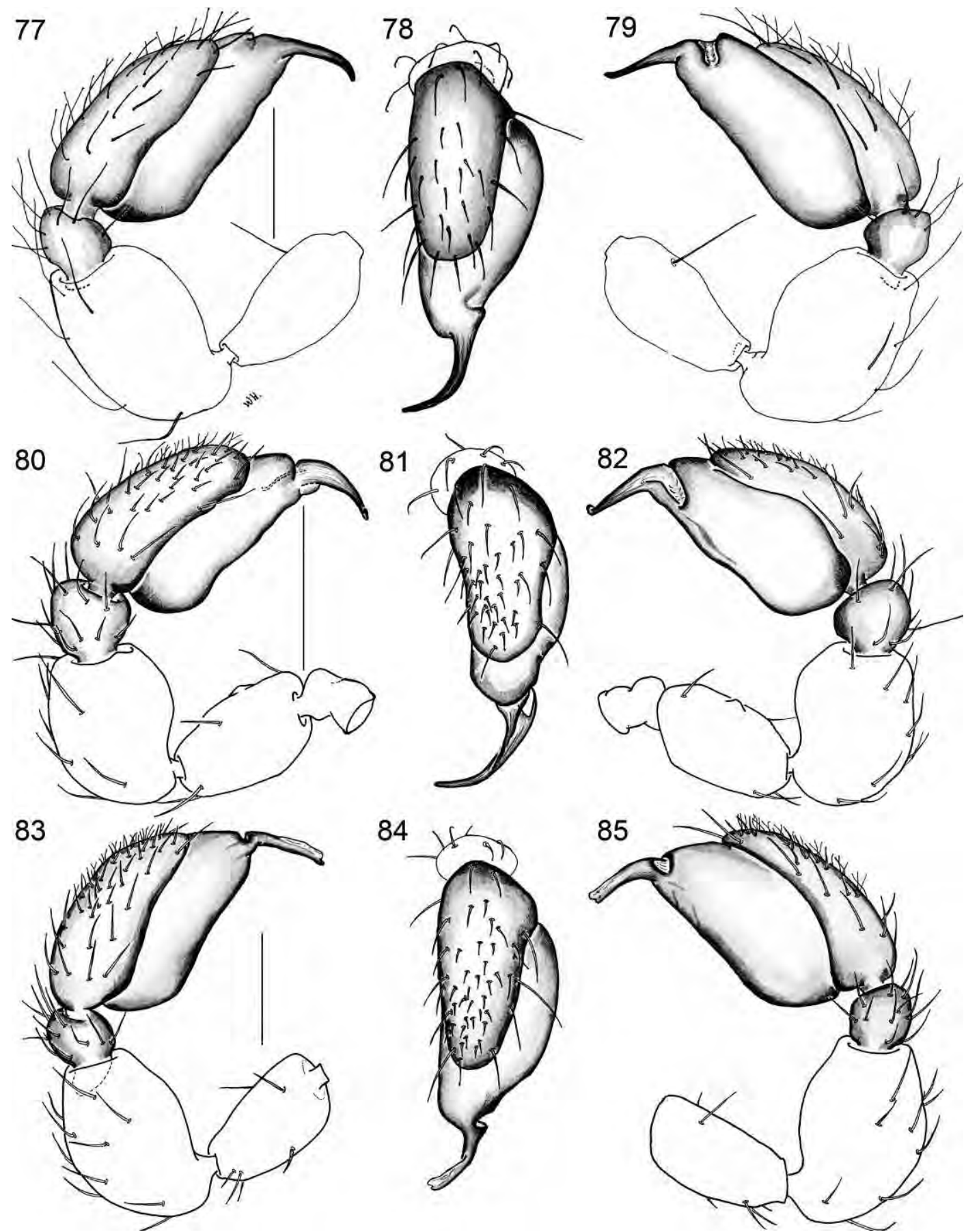

Figs. 77-85. Left palps, male: Prethopalpus schwendingeri, n. sp. (PBI_OON 16192), Prethopalpus perak, n. sp. (PBI_OON 15293), Prethopalpus kranzae, n. sp. (PBI_OON 12082). 77. P. schwendingeri, prolateral view. 78. Same, dorsal view. 79. Same, retrolateral view. 80. P. perak, prolateral view. 81. Same, dorsal view. 82. Same, retrolateral view. 83. P. kranzae, prolateral view. 84. Same, dorsal view. 85. Same, retrolateral view. 

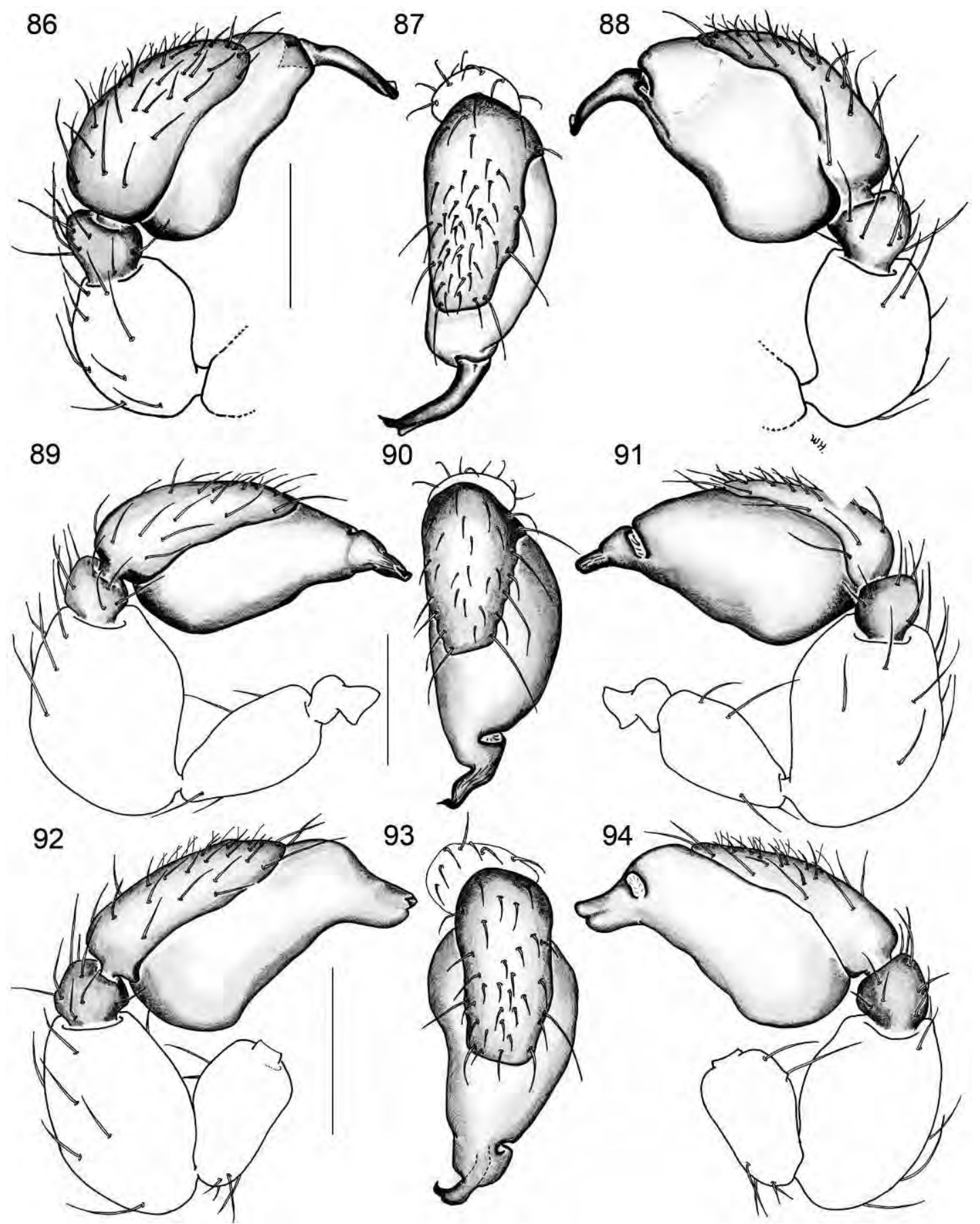

Figs. 86-94. Left palps, male: Prethopalpus pahang, n. sp. (PBI_OON 12123), Prethopalpus utara, n. sp. (PBI_OON 12085), Prethopalpus leuser, n. sp. (PBI_OON 12083). 86. P. pahang, prolateral view. 87. Same, dorsal view. 88. Same, retrolateral view. 89. P. utara, prolateral view. 90. Same, dorsal view. 91. Same, retrolateral view. 92. P. leuser, prolateral view. 93. Same, dorsal view. 94. Same, retrolateral view. 

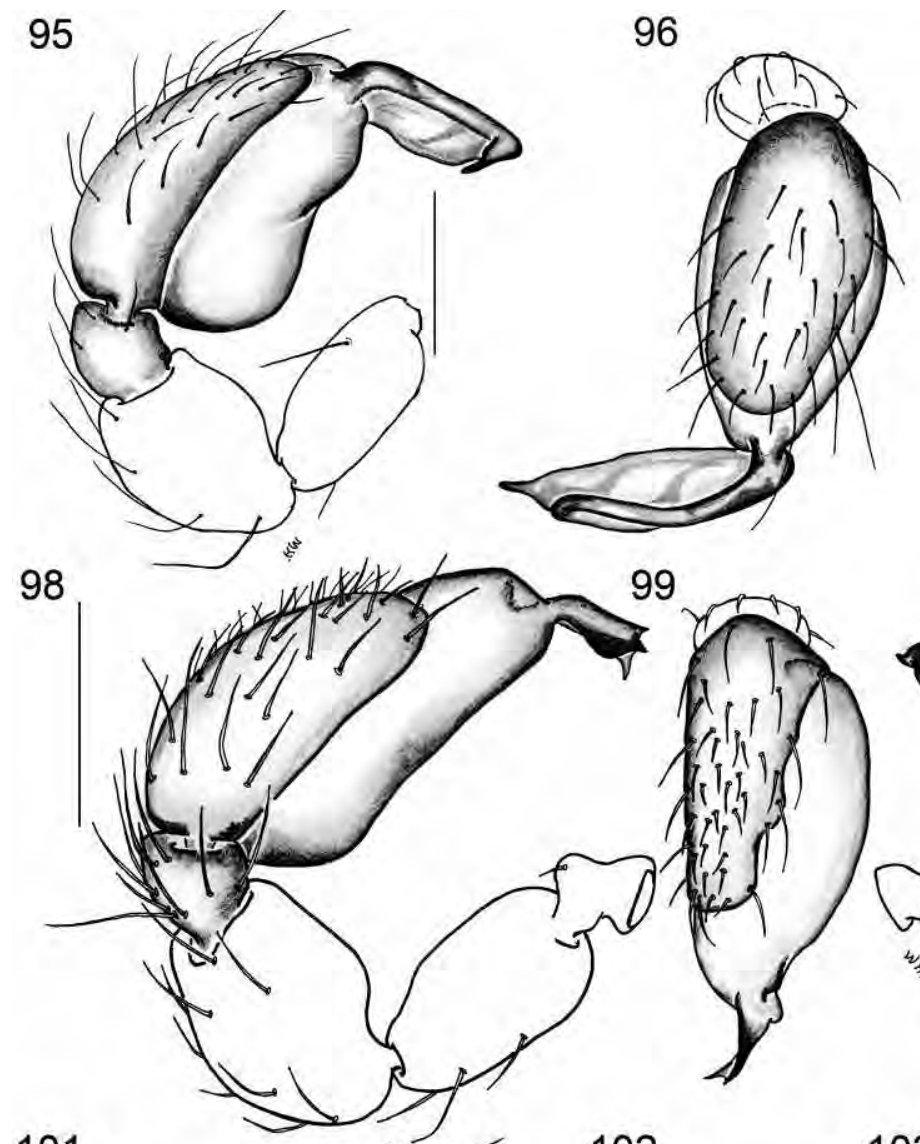

101

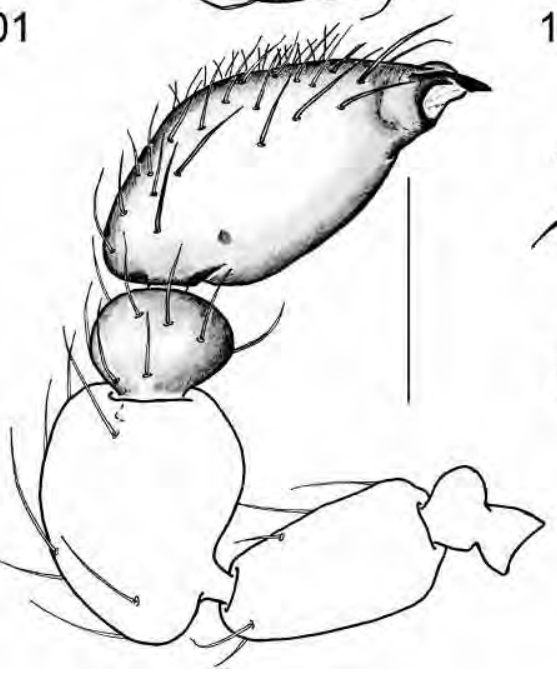

102

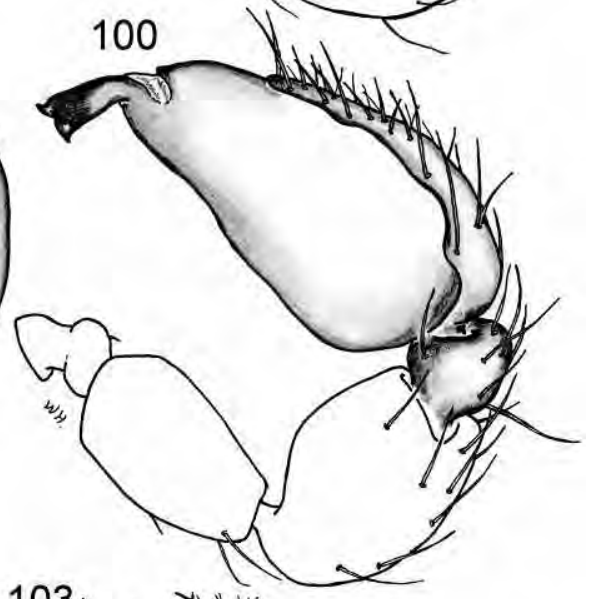

103

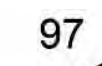



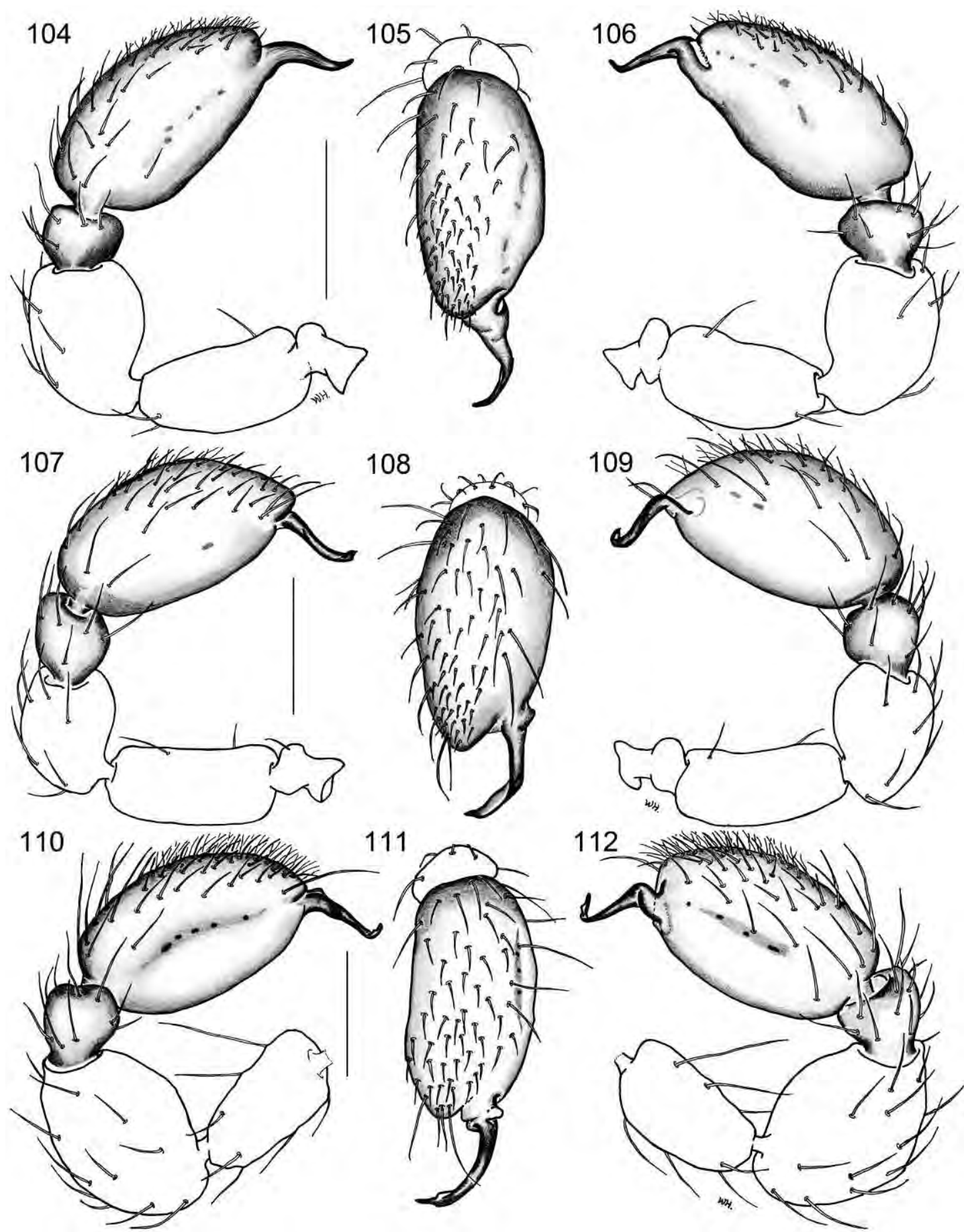

Figs. 104-112. Left palps, male: Prethopalpus kropfi, n. sp. (PBI_OON 12741), Prethopalpus sabah, n. sp. (PBI_OON 12344), Prethopalpus brunei, n. sp. (PBI_OON 15305). 104. P. kropfi, prolateral view. 105. Same, dorsal view. 106. Same, retrolateral view. 107. P. sabah, prolateral view. 108. Same, dorsal view. 109. Same, retrolateral view. 110. P. brunei, prolateral view. 111. Same, dorsal view. 112. Same, retrolateral view. 

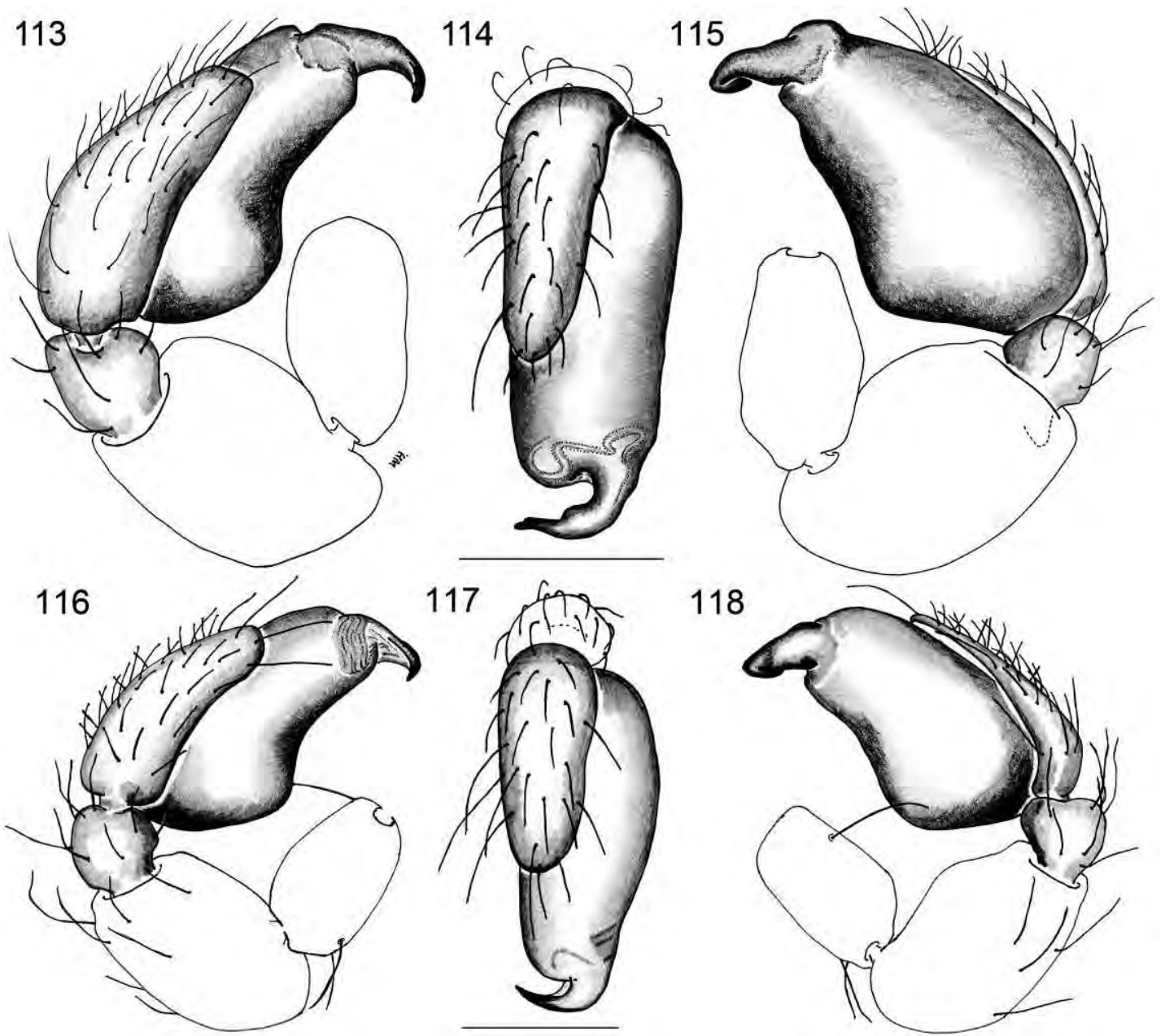

118
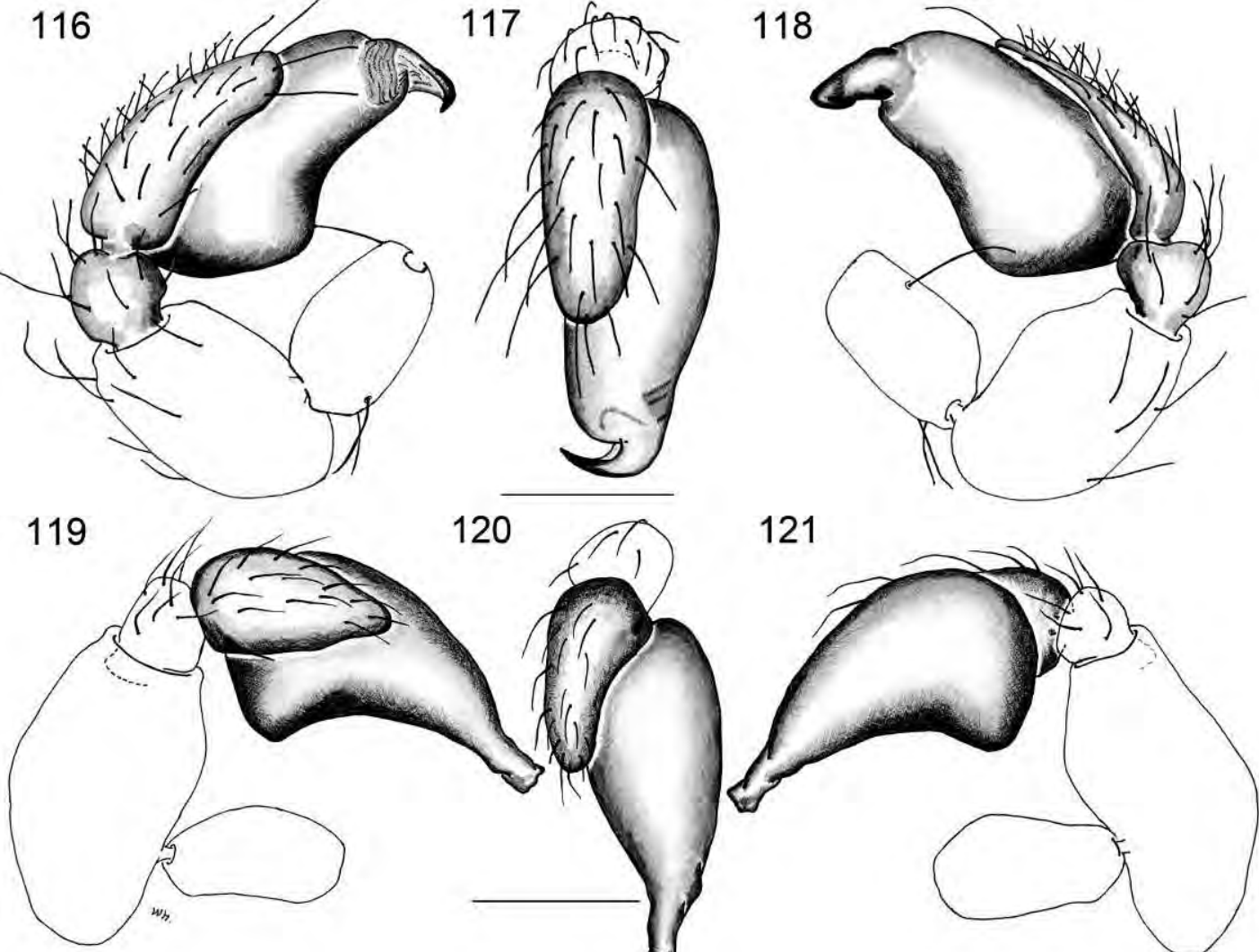

120

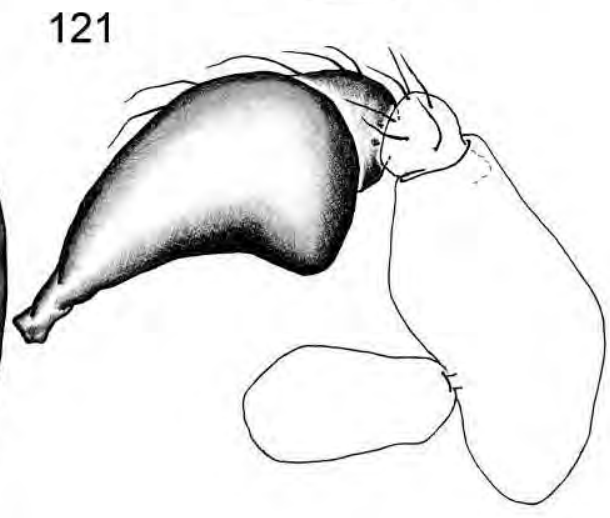

Figs. 113-121. Left palps, male: Prethopalpus attenboroughi, n. sp. (PBI_OON 07438), Prethopalpus platnicki, n. sp. (PBI_OON 21352), Prethopalpus blosfeldsorum, n. sp. (PBI_OON 06425). 113. P. attenboroughi, prolateral view. 114. Same, dorsal view. 115. Same, retrolateral view. 116. P. platnicki, prolateral view. 117. Same, dorsal view. 118. Same, retrolateral view. 119. P. blosfeldsorum, prolateral view. 120. Same, dorsal view. 121. Same, retrolateral view. 

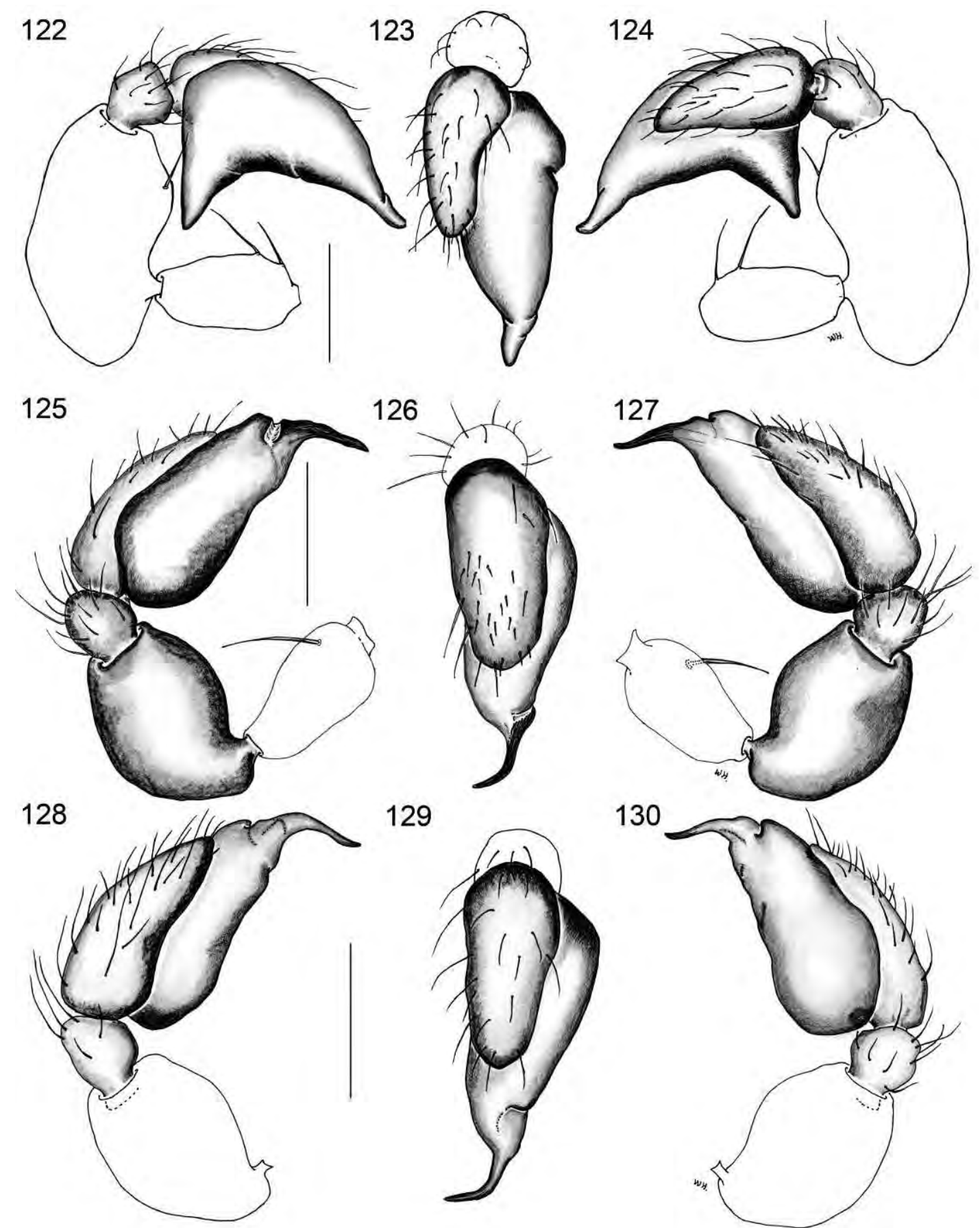

Figs. 122-130. Left palps, male: Prethopalpus rawlinsoni, n. sp. (PBI_OON 071129), Prethopalpus tropicus, n. sp. (PBI_OON 23339), Prethopalpus marionae, n. sp. (PBI_OON 23006). 122. P. rawlinsoni, prolateral view. 123. Same, dorsal view. 124. Same, retrolateral view. 125. P. tropicus, prolateral view. 126. Same, dorsal view. 127. Same, retrolateral view. 128. P. marionae, prolateral view. 129. Same, dorsal view. 130. Same, retrolateral view. 
131

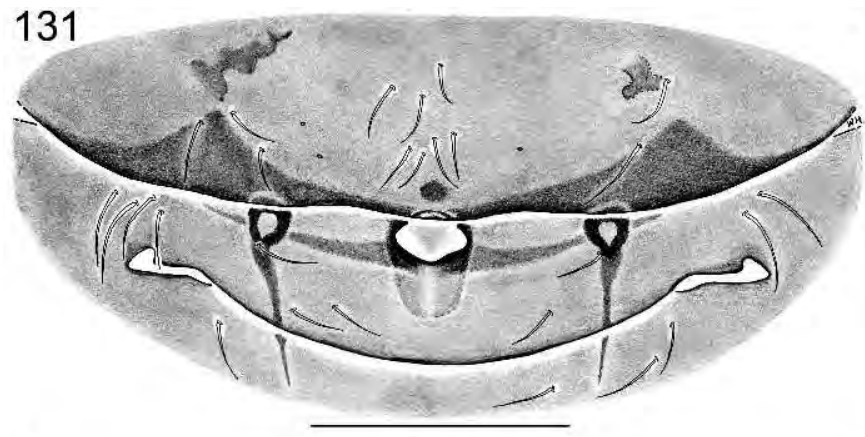

133

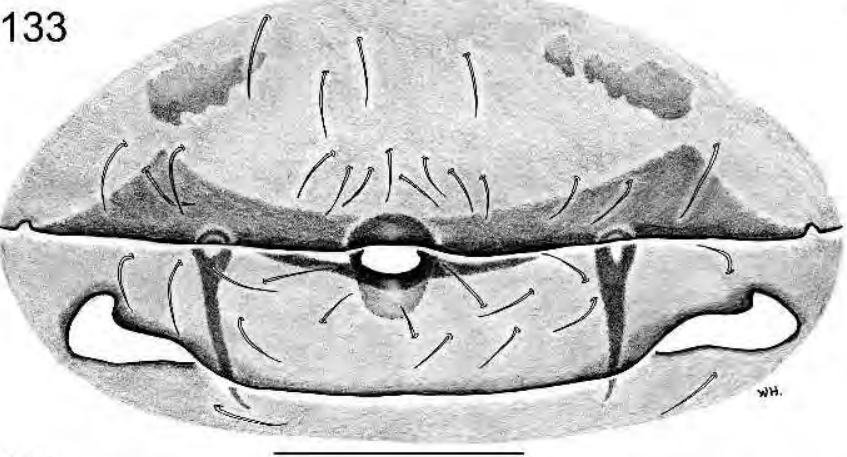

135

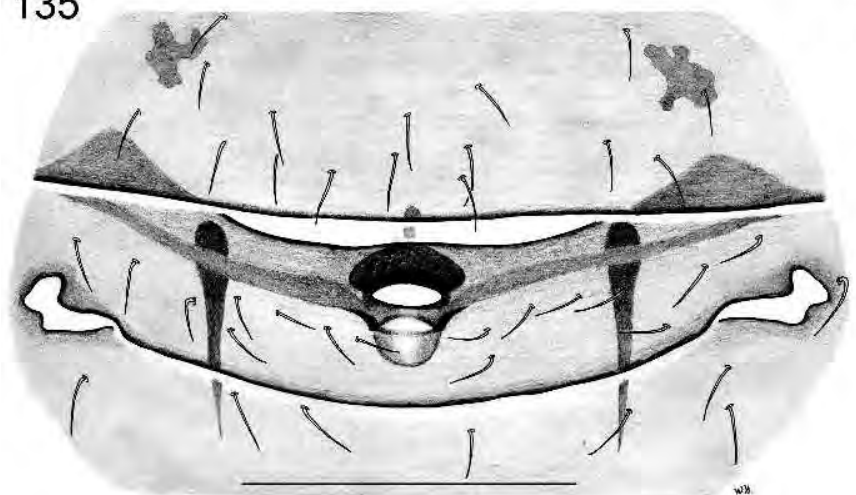

132

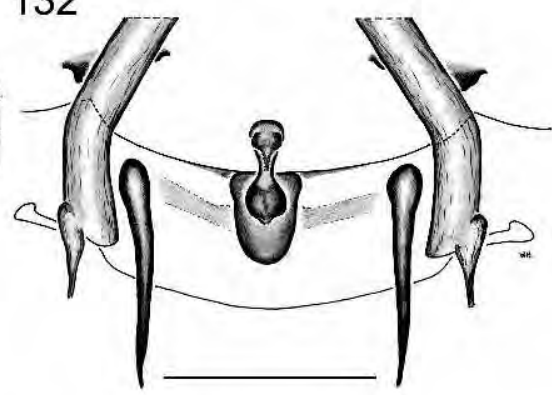

134

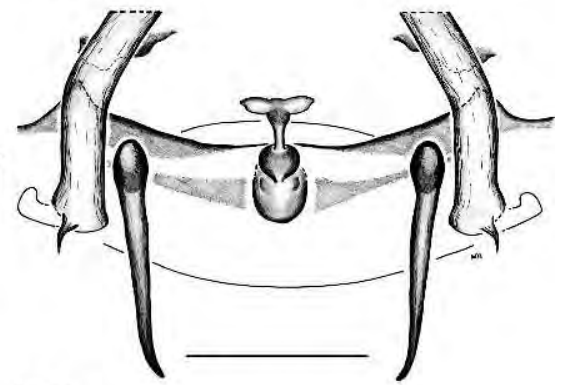

136

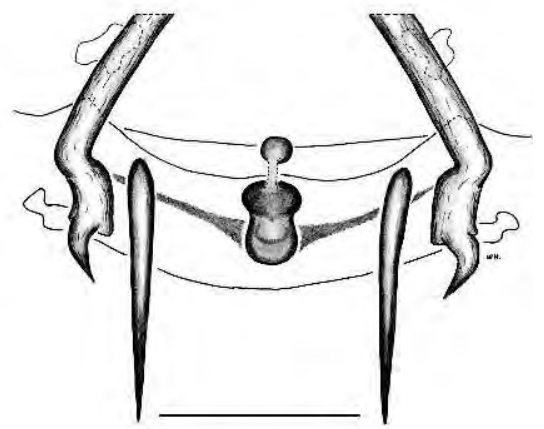

Figs. 131-136. Female epigyna. 131. Prethopalpus ilam, n. sp. (PBI_OON 23391), ventral view. 132. Same, dorsal view. 133. P. mahanadi, n. sp. (PBI_OON 12700), ventral view. 134. Same, dorsal view. 135. P. meghalaya, n. sp. (PBI_OON 15409), ventral view. 136. Same, dorsal view.

$\left[27.04000^{\circ} \mathrm{N}, 88.35000^{\circ} \mathrm{E}\right], 1400 \mathrm{~m}, 8$ Oct. 1978, C. Besuchet and I. Löbl, 1 ㅇ (MHNG, PBI_OON 00015500); District Darjeeling, Mahanadi près de Kurseong $\left[26.85500^{\circ} \mathrm{N}\right.$, $88.81200^{\circ} \mathrm{E}$, $1200 \mathrm{~m}, 6$ Oct. 1978 (C. Besuchet and I. Löbl, 2 s , 9 क (MHNG, PBI_OON 00012700, PBI_OON 00023386, PBI_OON 00015811).

Distribution: This species is known only from West Bengal in India (map 1).
Prethopalpus meghalaya Baehr, new species Figures 71-73, 135, 136, 219-237; map 1

TYPES: INDIA: Meghalaya: Male holotype from Khasi Hills, Mawphlang, $25.56666^{\circ} \mathrm{N}$, $91.88333^{\circ} \mathrm{E}, 1800 \mathrm{~m}, 28$ Oct. 1978 (C. Besuchet and I. Löbl), deposited in MHNG (PBI_OON 00015410). Female allotype collected with holotype, deposited in MHNG (PBI_OON 00015410). 


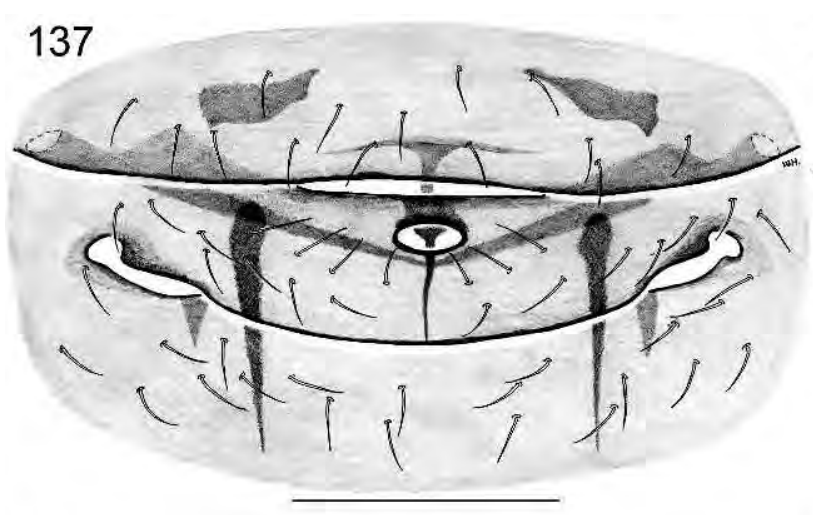

139

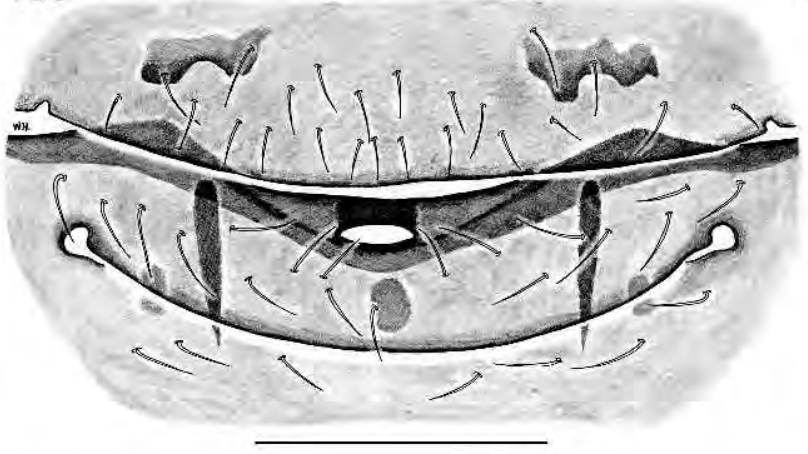

141

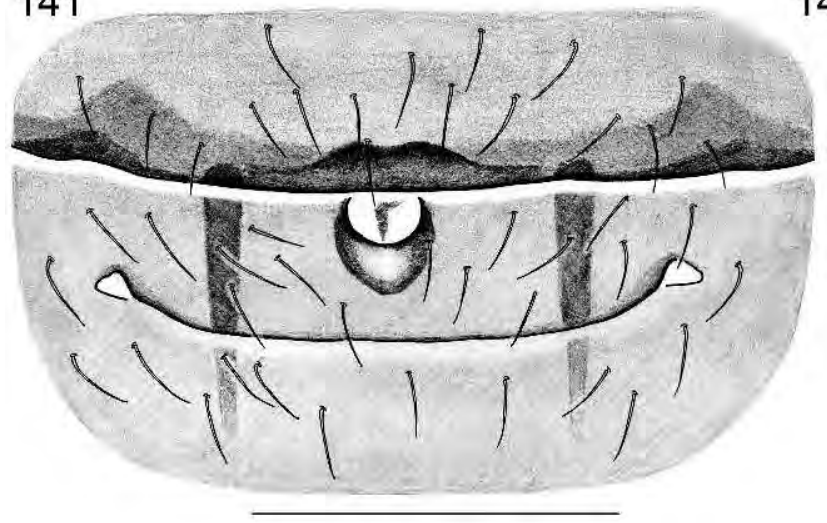

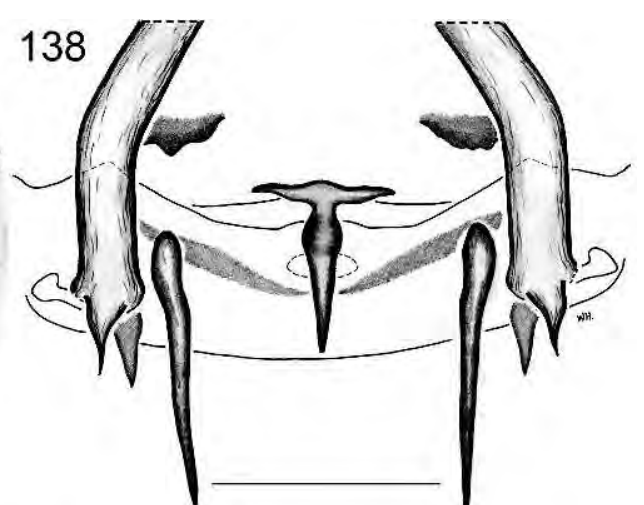

140
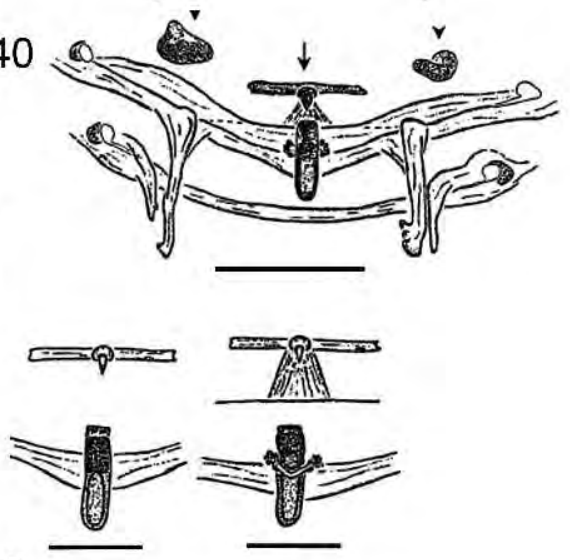

142

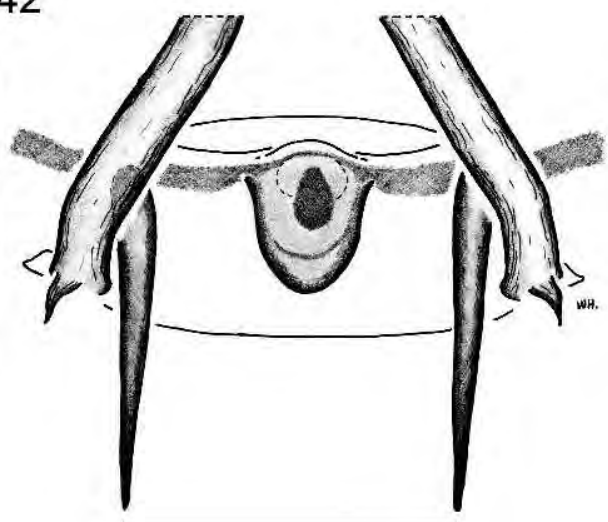

Figs. 137-142. Female epigyna. 137. Prethopalpus utara, n. sp. (PBI_OON 23392), ventral view. 138. Same, dorsal view. 139. P. fosuma (Burger, Nentwig, and Kropf) (PBI_OON 12377), ventral view. 140. Same, dorsal view, including detail of vulva, dorsal view (bottom left) and ventral view (bottom right) (from Burger et al. 2002) (arrow $=$ sclerite bearing naillike structure; arrowheads $=$ sclerites functioning as muscle attachments). 141. P. leuser, n. sp. (PBI_OON 23397), ventral view. 142. Same, dorsal view.

ETYMOLOGY: The specific name is a noun in apposition taken from the type locality.

DiAgnosis: Males resemble those of $P$. $k h a s i$, also from India, in general body shape and by lacking a palpal conductor, but can be easily recognized by having a recurved posterior eye row (figs. 219, 224), a larger palpal patella, and a marginally sclerotized embolus 

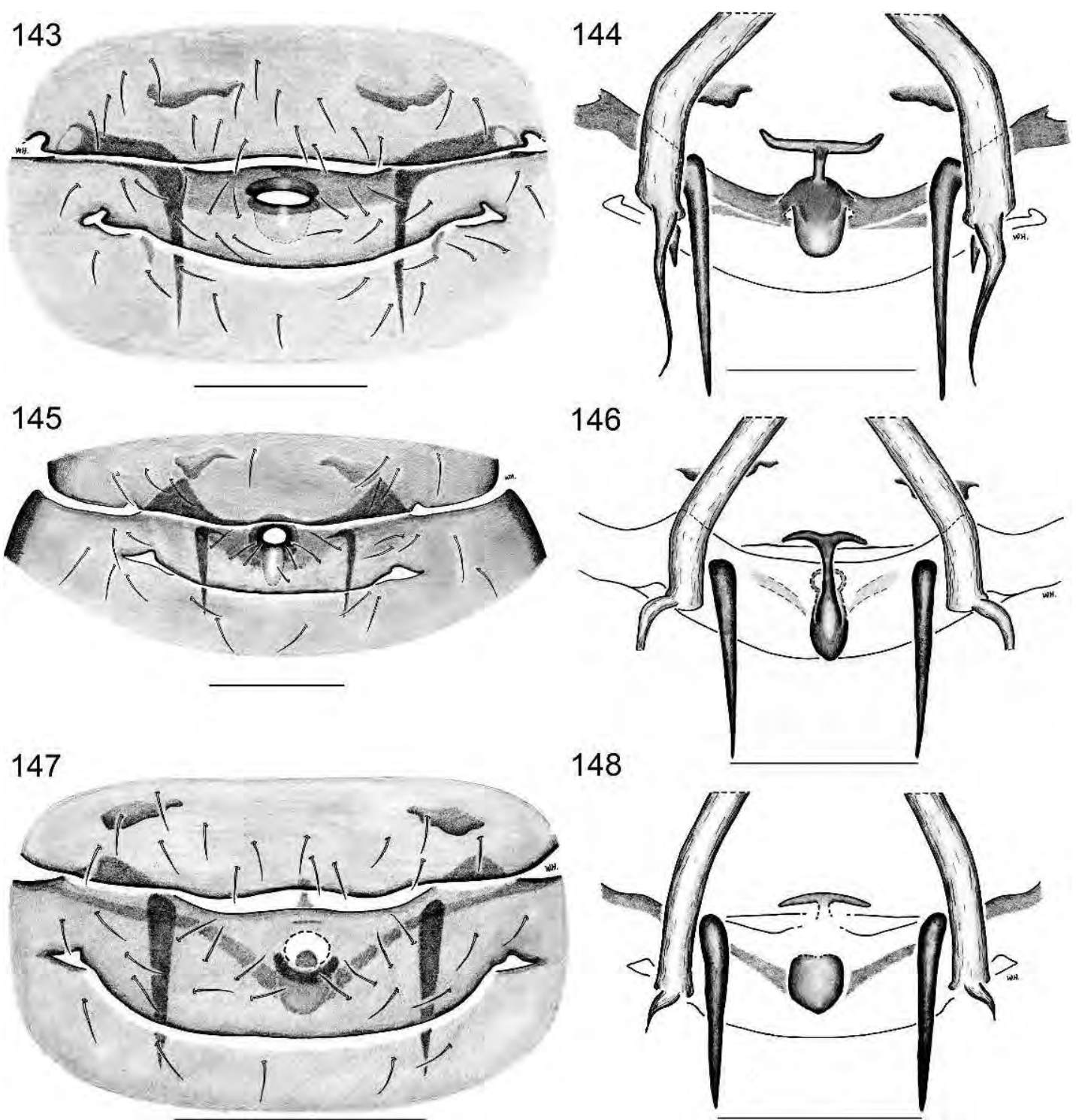

Figs. 143-148. Female epigyna. 143. Prethopalpus schwendingeri, n. sp. (PBI_OON 12426), ventral view. 144. Same, dorsal view. 145. P. brunei, n. sp. (PBI_OON 16225), ventral view. 146. Same, dorsal view. 147. P. java, n. sp. (PBI_OON 16211), ventral view. 148. Same, dorsal view.

(figs. 71-73). Females can be separated from those of all other Indian species by having the epigynal opening broadly oval with an apical sclerotized hood (fig. 135), internal genital structure long and oval, medially constricted, with circular anterior receptaculum (figs. 135, 136).

MALE (PBI_OON 15410, figs. 219-228): Total length 1.77. Prosoma, mouthparts, and abdominal scuta pale orange, elevated por- tion of pars cephalica smooth, sides granulate; lateral margin without denticles. Eyes: ALE 0.062; PME 0.054; PLE 0.051; ALE largest, ALE circular, PME oval, PLE circular; posterior eye row recurved from above, straight from front; ALE separated by less than their radius, ALE-PLE separated by less than ALE radius, PME touching for less than half their length, PLE-PME separated 

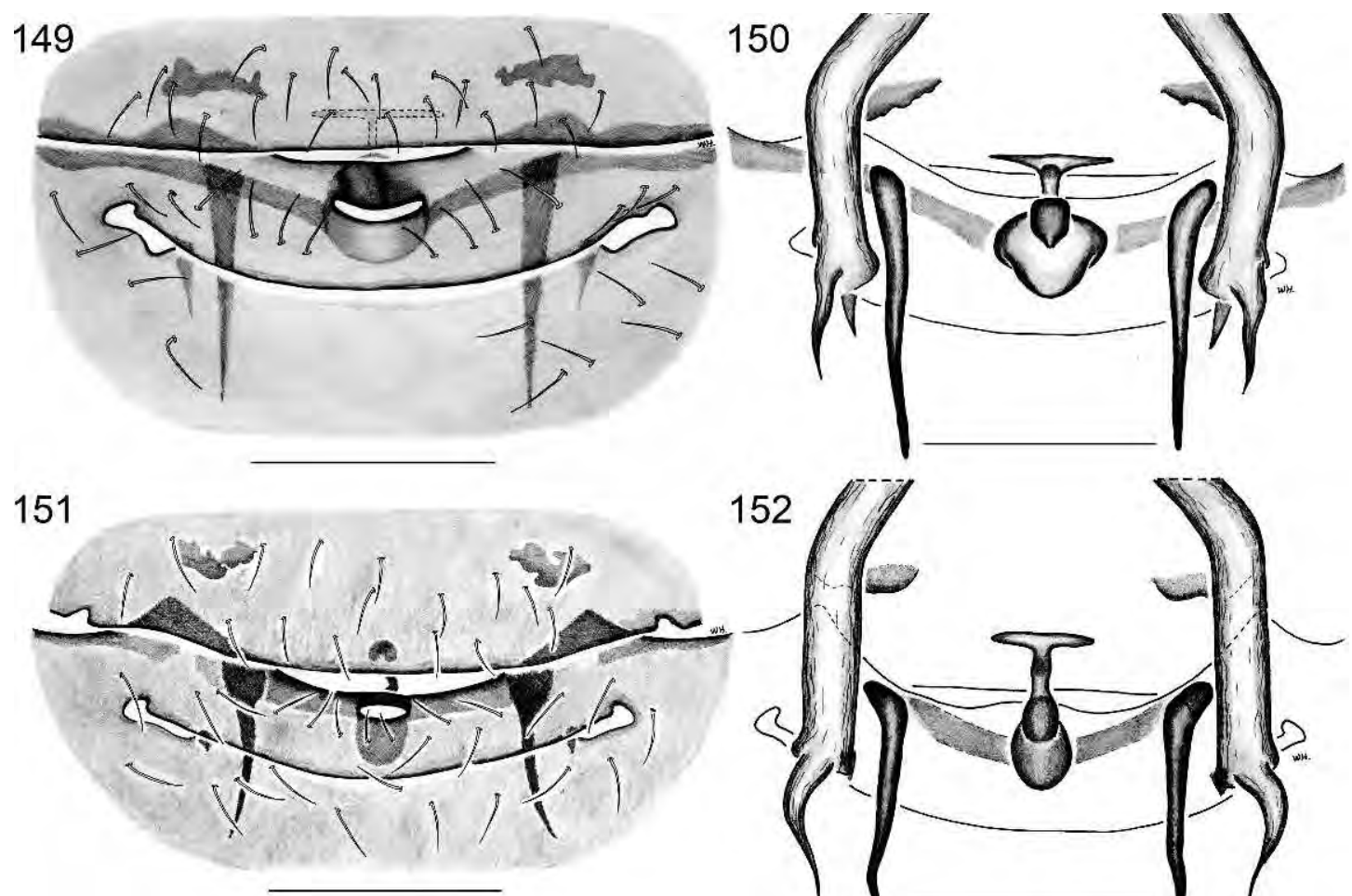

153

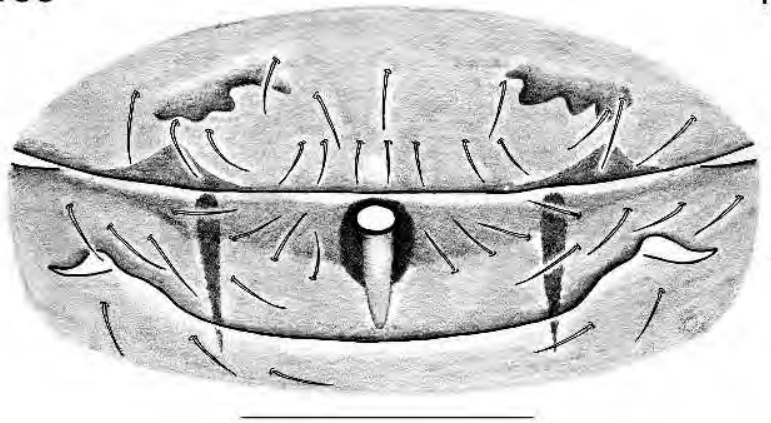

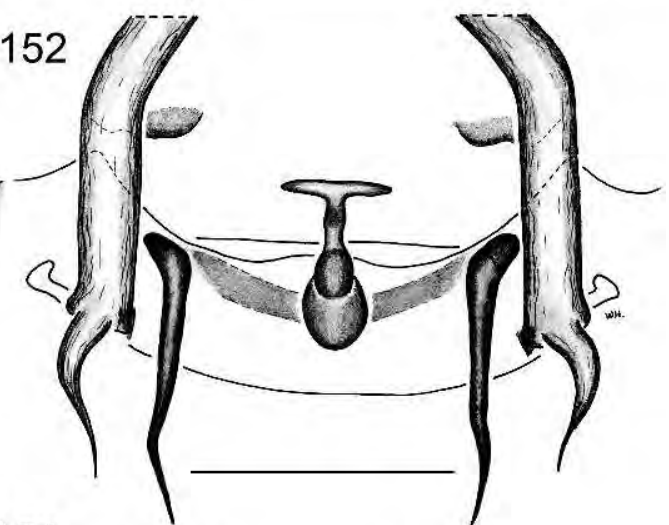

154

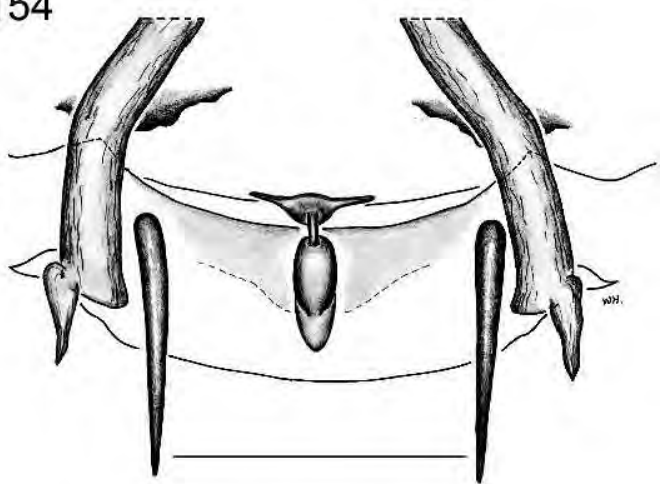

Figs. 149-154. Female epigyna. 149. Prethopalpus sabah, n. sp. (PBI_OON 12313), ventral view. 150. Same, dorsal view. 151. P. peraki, n. sp. (PBI_OON 12515), ventral view. 152. Same, dorsal view. 153. $P$. kropfi, n. sp. (PBI_OON 23394), ventral view. 154. Same, dorsal view.

by less than PME radius. Sternum longer than wide, surface smooth, covered with small round pits. Endites about 1.5 times as long as wide, with short, square toothlike projection. Abdomen ovoid; book lung covers large; pedicel tube short, ribbed. Dorsal scutum covering full length of abdomen, smooth; epigastric scutum not protrud- ing; postepigastric scutum, long, almost rectangular, covering nearly full length of abdomen. Legs yellow; patella plus tibia I nearly as long as carapace. Palp yellow, embolus dark; femur attaching to patella subbasally; patella about as long as femur, enlarged; cymbium not fused with bulb, bulb pyriform distally with triangular, medially 
bent embolus with dark sclerotized margins (viewed laterally) (figs. 71-73).

Female (PBI_OON 15409, figs. 229237): Total length 1.62. Eyes: ALE 0.044; PME 0.038; PLE 0.032. Epigastric area with epigynal opening broadly oval and apical sclerotized hood; internal genital structure long, oval, and medially constricted; anterior receptaculum circular (figs. 135, 136).

OTHER MATERIAL EXAMINED: INDIA: Assam: Manas $\left[26.72500^{\circ} \mathrm{N}, 91.03600^{\circ} \mathrm{E}\right]$, $200 \mathrm{~m}, 21-22$ Oct. 1978, C. Besuchet and I. Löbl, 1 के (MHNG, PBI_OON 00015391). Meghalaya: Garo-Hills, above Tura $\left[25.56000^{\circ} \mathrm{N}\right.$, $\left.89.49000^{\circ} \mathrm{E}\right], 700 \mathrm{~m}, 1$ Nov. 1978, C. Besuchet and I. Löbl, 1 § , 2 + (MHNG, PBI_OON 00015409); Khasi Hills, au-dessus de Shillong, $25.56666^{\circ} \mathrm{N}$, $91.88333^{\circ} \mathrm{E}, 1850 \mathrm{~m}, 25$ Oct. 1978, D. Burckhardt and I. Löbl, 1 đ̊ (MHNG, PBI_OON 00012714); same location, 30 Oct. 1978, C. Besuchet and I. Löbl, 1 के (MHNG, PBI_OON 00012686).

DisTRIBUTION: This species in known from Assam and Meghalaya districts in India (map 1).

\section{SPECIES FROM INDONESIA, MALAYSIA, SINGAPORE, AND BRUNEI}

\section{Key to Species}

1. Males................... 2 Females (unknown for $P$. bali, $P$. deelemanae, $P$. kranzae, $P$. magnocularis, $P$. pahang and $P$. sarawak). . . . . . . . . . . 17

2. Eyes reduced (fig. 339); palpal bulb bulging dorsodistally (figs. 92, 94, 341, 343) . . . P. leuser Eyes well developed (e.g., figs. 6, 311); palpal bulb not bulging dorsodistally (e.g., figs. 8 , 107) . . . . . . . . . . . 3

3. Cymbium and bulb completely separated (e.g., figs. 95-100) . . . . . . . . . . . 4 Cymbium and bulb only partly separated marked by pits (figs. 102-112) . . . . . . 12

4. Embolar region with conductor and ventral apophysis (fig. 255) . . . . . . P. bellicosus Embolar region lacking conductor and apophysis (figs. 77, 100). . . . . . . 5

5. Embolus as long as bulb, with velum (figs. 95-97) ........... . P. sarawak Embolus shorter, without velum (figs. 77-91, 98-100) . . . . . . . . . . 6

6. Carapace strongly granulate (e.g., figs. 311, $312,376,377) \ldots \ldots \ldots \ldots$
Carapace smooth, finely reticulate, or finely granulate (e.g., figs. 243, 244) . . . . . . 8

7. Carapace posterolateral surface with a pair of spikes, lateral margin with sharply pointed denticles (figs. 311, 312) . . .... P. kranzae Carapace without spikes or sharply pointed denticles (figs. 376, 377) . . . . . . P. perak

8. Embolar tip bifid (e.g., figs. 98-100) .... 9

- Embolar tip pointed (e.g., figs. 77-79). . . 10

9. Embolus short, more or less straight in dorsal view (fig. 99, 246) . . . . . . . . . . . P. bali Embolus longer, bent medially (fig. 87) . . . . . . ............. P. pahang

10. Embolus short, broad, medially bent (fig. 93) .............. P. utara Embolus long and thin (figs. 77-79) ... . 11

11. Carapace sides with three upturned granulate patches (figs. 7, 20) . . . . . P. fosuma Carapace sides continuously granulate (fig. 425, 426) . . . . . . . P. schwendingeri

12. Sternum with whole surface strongly reticulate (e.g., fig. 393) . . . . . . . . . . . . 13 Sternum with sides strongly reticulate and centre smooth (e.g., fig. 320) . . . . . . . 15

13. Palpal femur about twice as long as patella (figs. 107, 109) ............ . P. sabah Palpal femur shorter (figs. 113, 115) . . . 14

14. Bulbal tip broad with ventral concavity, embolus short (figs. 101-103) . . . . P. java Bulbal tip ending in thin, medially bent embolus (figs. 110-112) . . . . . . . P. brunei

15. Sternum with median smooth area shaped like an inverted drop (fig. 320) .. . P. kropfi Sternum with median smooth area a longitudinal band (e.g., fig. 284) . . . . . . . . . 16

16. Eyes extremely large (figs. $353,356,357) \ldots \ldots$. . ............ P. magnocularis Eyes much smaller (fig. 283) . . P. deelemanae

17. Eyes reduced (fig. 349); epigastric area with procurved sclerotized area posterior to genital opening (fig. 141) . . . . . . . . P. leuser

- Eyes well developed; epigastric area without procurved sclerotized area (e.g., fig. 258) . . 18

18. Sternum with median area smooth and shaped like an inverted drop (fig. 261) . . 19 Sternum with entire surface reticulate (e.g., figs. 301, 383, 404) . . . . . . . . . 21

19. Epigynal opening extremely wide and procurved (fig. 258) . . . . . . . . P. bellicosus Epigynal opening much smaller and not procurved (figs. 281, 329) . . . . . . . . . 20

20. Internal genital structure with anchor-shaped sclerite anteriorly (fig. 146). . . . . P. brunei Internal genital structure with $\mathrm{T}$-shaped sclerite anteriorly (fig. 154). . . . . P. kropfi

21. Carapace sides strongly striated, top punctate (fig. 303). . . . . . . . . . . P. java 
Carapace granulate or smooth (e.g., figs. 42, 388) . . . . . . . . . . . . 22

22. Carapace sides and top granulate (fig. 388). . . . . . P. perak Carapace at least top smooth (e.g., fig. 405) . . . 23

23. Carapace sides and top smooth, only few rows of punctation (figs. 405, 406); epigynal opening broad and slitlike (figs. 149, 408) . . . . .

P. sabah Carapace sides granulate, top smooth (e.g., fig. 41); epigynal opening small, oval (e.g., fig. 137) . . . . . . . . . . . . . 24

24. Carapace sides with three almost recurved granulate patches (fig. 42) . . . . P. fosuma Carapace sides continuously granulate (figs.

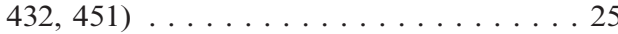

25. Internal genital structure spinelike, nearly reaching tracheal groove (fig. 138) . . . . . P. utara Internal genital structure short and rounded, not reaching tracheal groove (fig. 144). . . . .

P. schwendingeri

Prethopalpus bali Baehr, new species

Figures 98-100, 238-247; map 2

TYPE: INDONESIA: Bali: Male holotype from northern Bali, between Busungbiu and Pupuan, $8.30110^{\circ} \mathrm{S}, 114.95330^{\circ} \mathrm{E}, 650 \mathrm{~m}(19$ 22 Dec. 2007, A. Schultz), deposited in MHNG (PBI_OON 00016128).

ETYMOLOGY: The specific name is a noun in apposition taken from the type locality.

Diagnosis: Males resemble those of $P$. pahang in body shape and having a bipartite embolar tip, but can be easily recognized by the short, more or less straight, bipartite embolus in dorsal view (fig. 99).

Male (PBI_OON 16128, figs. 238-244): Total length 1.12. Prosoma, mouthparts, and abdominal scuta pale orange, surface of elevated portion of pars cephalica smooth, sides granulate; lateral margin without denticles. Eyes small, ALE 0.039; PME 0.024; PLE 0.023, ALE circular, PME oval, PLE circular; posterior eye row straight from above, straight from front; ALE separated by their radius to diameter, ALE-PLE separated by less than ALE radius, PME touching for less than half their length, PLEPME separated by less than PME radius. Sternum longer than wide, surface not finely reticulate, without pits. Endites about twice as long as wide; toothlike projection hook shaped and bent medially. Abdomen ovoid; book lung covers large; pedicel tube short, ribbed. Dorsal scutum covering full length of abdomen, smooth; epigastric scutum not protruding; postepigastric scutum, long, almost rectangular, covering nearly full length of abdomen, with long posteriorly directed lateral apodemes. Legs yellow; patella plus tibia I shorter than carapace. Epigastric region with oval sperm pore oval. Palp yellow; embolus light; femur attaching to patella subbasally; patella about as long as femur, enlarged; cymbium not fused with bulb, pyriform with bipartite, medially bent embolus (figs. 98-100, 245-247).

FEMALE: Unknown.

Other Material Examined: None.

DisTRIBUTION: This species is known only from Bali (map 2).

\section{Prethopalpus bellicosus Baehr and Thoma, new species}

Figures 248-263; map 5

TYPE: MALAYSIA: Sabah: Male holotype from Crocker Range, $5.66666^{\circ} \mathrm{N}, 116.33333^{\circ} \mathrm{E}$, $1600 \mathrm{~m}$ (16 May 1987, D. Burckhardt and I. Löbl), deposited in MHNG (PBI_OON 00012353).

ETYMOLOGY: The specific name bellicosus is Latin suggested by the heavily sclerotized scuta that resemble strong armature and a ready means for fighting.

DiAGNOSIS: Males can be easily separated from those of all other species by the palpal bulb with a conductor and an additional ventral apophysis (fig. 255), and females by an extremely wide and procurved epigynal opening (fig. 258).

Male (PBI_OON 12353, figs. 248-254): Total length 1.26. Prosoma, mouthparts, and abdominal scuta orange-brown, broadly oval in dorsal view, pars cephalica strongly elevated in lateral view, posterolateral edge without pits, surface uniformly granulate except clypeus and area anterior to eye group; lateral margin with sluice reaching clypeus, without denticles; nonmarginal pars cephalica setae with three pairs of enlarged setae, one on clypeus (lateral setae), one (very thick hairs, close together) anterior to eye group, pointing anteriorly and a pair of smaller hairs between ALEs, pointing upward; marginal setae few. Clypeus sinuous in front view, sloping forward in lateral view, 


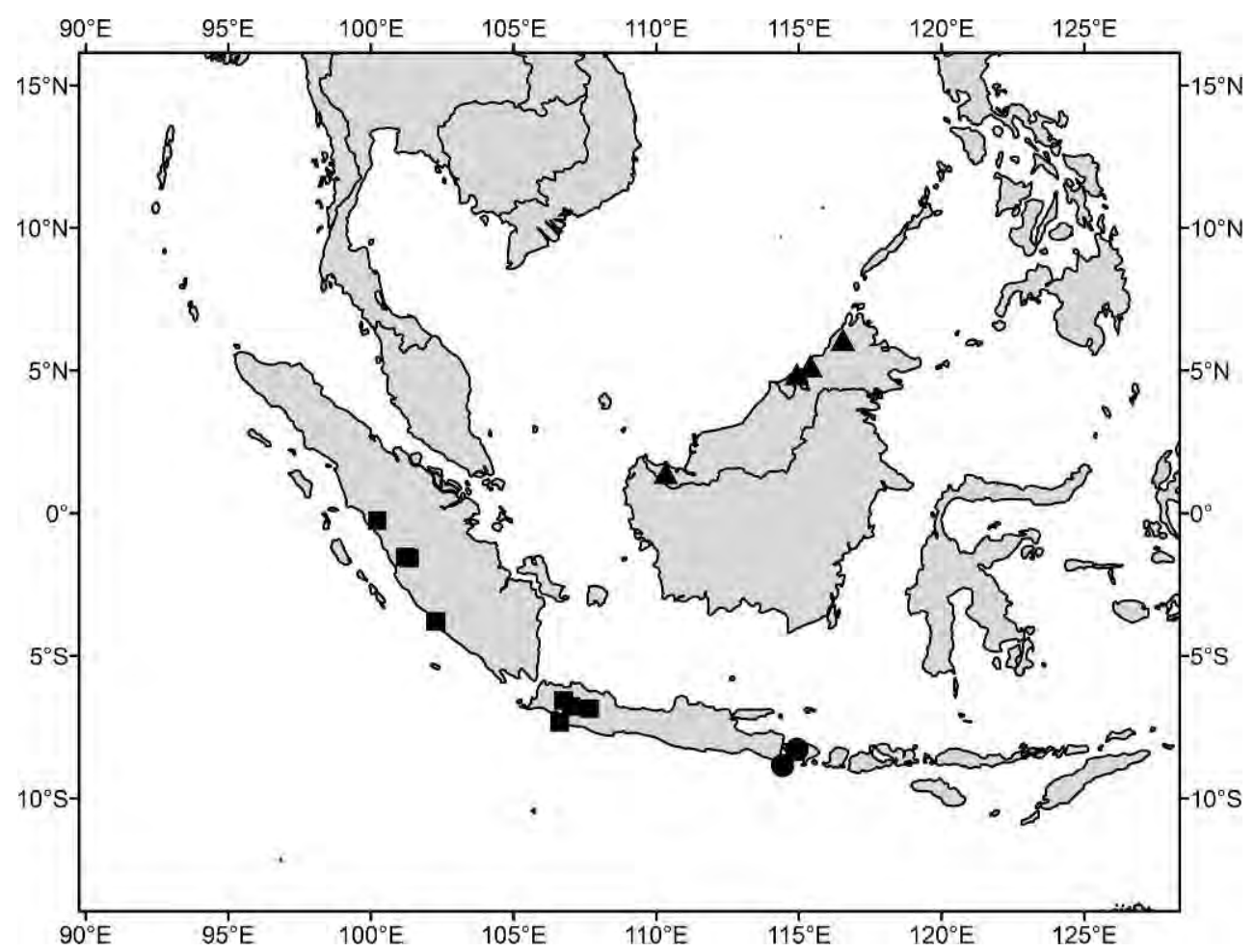

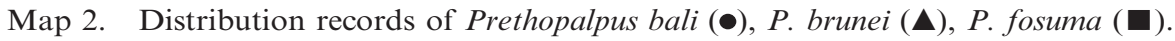

distance ALE to clypeus margin about $2.5 \times$ ALE diameter. Eyes: ALE, PME subequal, larger than PLE, PME circular; posterior eye row straight from above; ALE separated by more than their diameter, PLE-PME separated by $P M E$ radius to $P M E$ diameter. Sternum only slightly longer than wide; radial furrows weakly developed; infracoxal grooves present with anterior and posterior depression; with radial furrows between coxae I-II, II-III, III-IV, microsculpture only medially, setae evenly scattered. Chelicerae anterior face proximal part slightly convex in lateral view, laminate groove only weakly developed; setae few scattered setae on anterior face of paturon. Labium shape trapezoidal, anterior margin indented at middle; 2 setae on anterior margin (one on each side of indention) and 3 subdistal setae. Abdomen oval, book lung covers ovoid; dorsal of pedicel tube with a pair of narrow elongated cuticular outgrowths. Dorsal scutum pits surrounding hair bases causing punctate impression; postepigastric scutum long, semicircular. Legs pale orange, femora and basal half of tibiae darkened; about proximal half of femora darkened. Epigastric region with sperm pore situated between anterior and posterior spiracles. Palp orange-brown; embolus dark; trochanter with ventral projection; femur attaching to patella subbasally; patella longer than femur, slightly larger than femur; tibia stout, rounded, about 5 times shorter than patella; cymbium not fused to bulb; bulb with conductor and ventral apophysis (fig. 255).

Female (PBI_OON 12353, figs. 256263): Total length 1.30. Epigastric area with extremely wide, procurved, epigynal opening (fig. 258).

Other Material Examined: MALAYSIA: Sabah: Mt. Kinabalu, $6.08333^{\circ} \mathrm{N}$, $116.5500^{\circ} \mathrm{E}, 1500 \mathrm{~m}, 25$ Apr. 1987, D. Burckhardt and I. Löbl, 1 के (MHNG, PBI_OON 00012221); same location, 30 Apr. 1987, D. Burckhardt and I. Löbl, 1 s (MHNG, PBI_OON 00012275); same location, 21 May 1987, D. Burckhardt and I. 
155

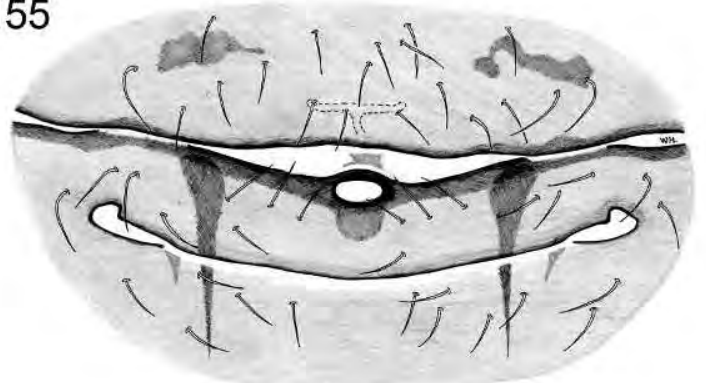

157
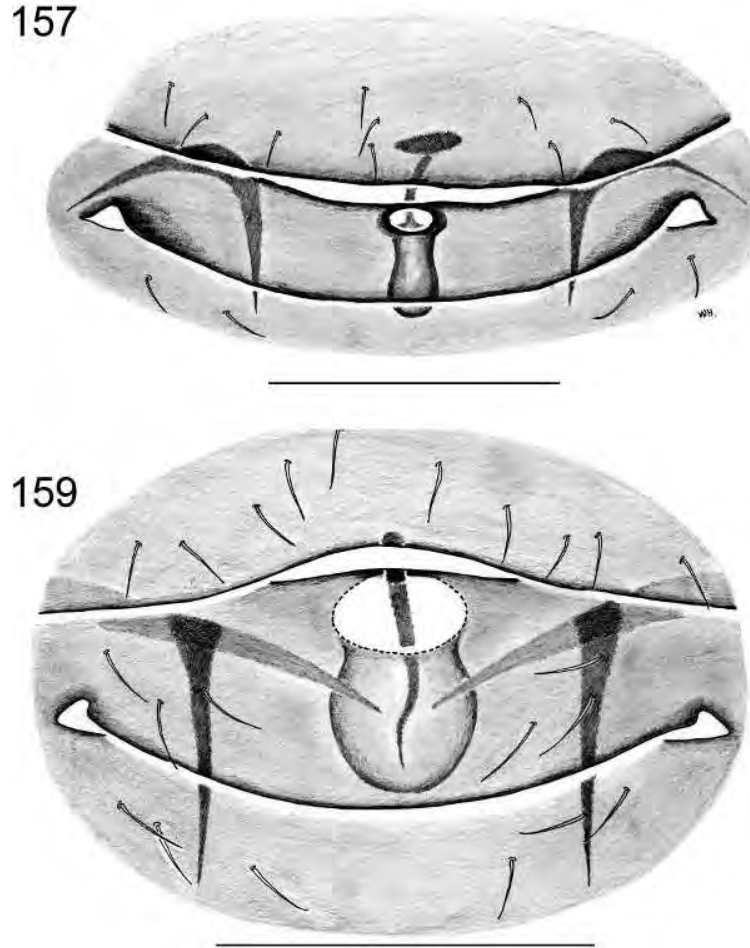

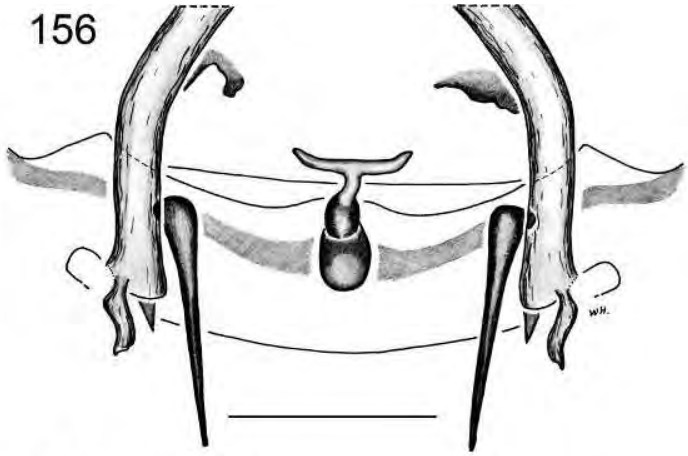

158

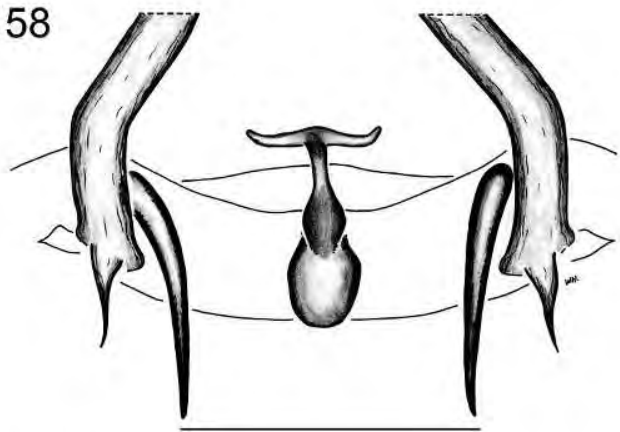

160

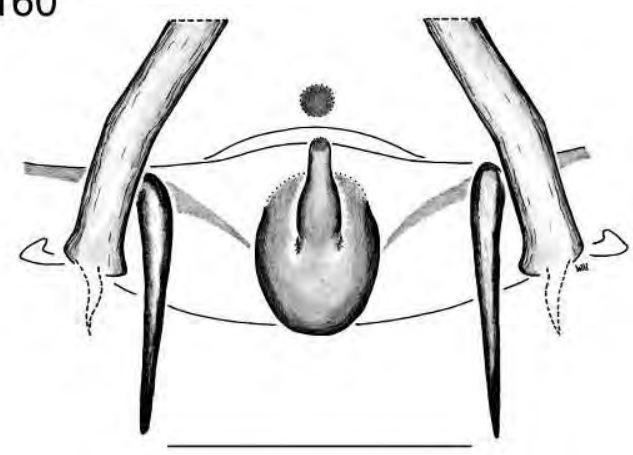

Figs. 155-160. Female epigyna. 155. Prethopalpus tropicus, n. sp. (PBI_OON 23373), ventral view. 156. Same, dorsal view. 157. P. marionae, n. sp. (PBI_OON 23403), ventral view. 158. Same, dorsal view. 159. P. platnicki, n. sp. (PBI_OON 21352), ventral view. 160. Same, dorsal view.

Löbl, 2 के (MHNG, PBI_OON 00012287, PBI_OON 00012368); Mt. Kinabalu, $6.08333^{\circ} \mathrm{N}, 116.55^{\circ} \mathrm{E}, 1550 \mathrm{~m}, 24$ Apr. 1987 , D. Burckhardt and I. Löbl, 2 के (MHNG, PBI_OON 00012259, PBI_OON 00012355); same location, 28 Apr. 1987, D. Burckhardt and I. Löbl, 1 s (MHNG, PBI_OON 00012370); same location, $1430 \mathrm{~m}, 22$ May 1987, D. Burckhardt and I. Löbl, 1 f (MHNG, PBI_OON 00012222); Mt Kinabalu, Park Headquarters, Liwagu River,
1490 m, 18 May 1987, A. Smetana, 1 के (MHNG, PBI_OON 00012193); Mt Kinabalu National Park, Poring Hot Spring, at Langanan Creek, 915 m, 18 Aug. 1988, A. Smetana, 1 के (MHNG, PBI_OON 00012699); Poring Hot Springs, 500 m, 11 May 1987, D. Burckhardt and I. Löbl, 1 sे (MHNG, PBI_ OON 00012357).

Distribution: Prethopalpus bellicosus is known only from Sabah in Malaysia (map 5). 


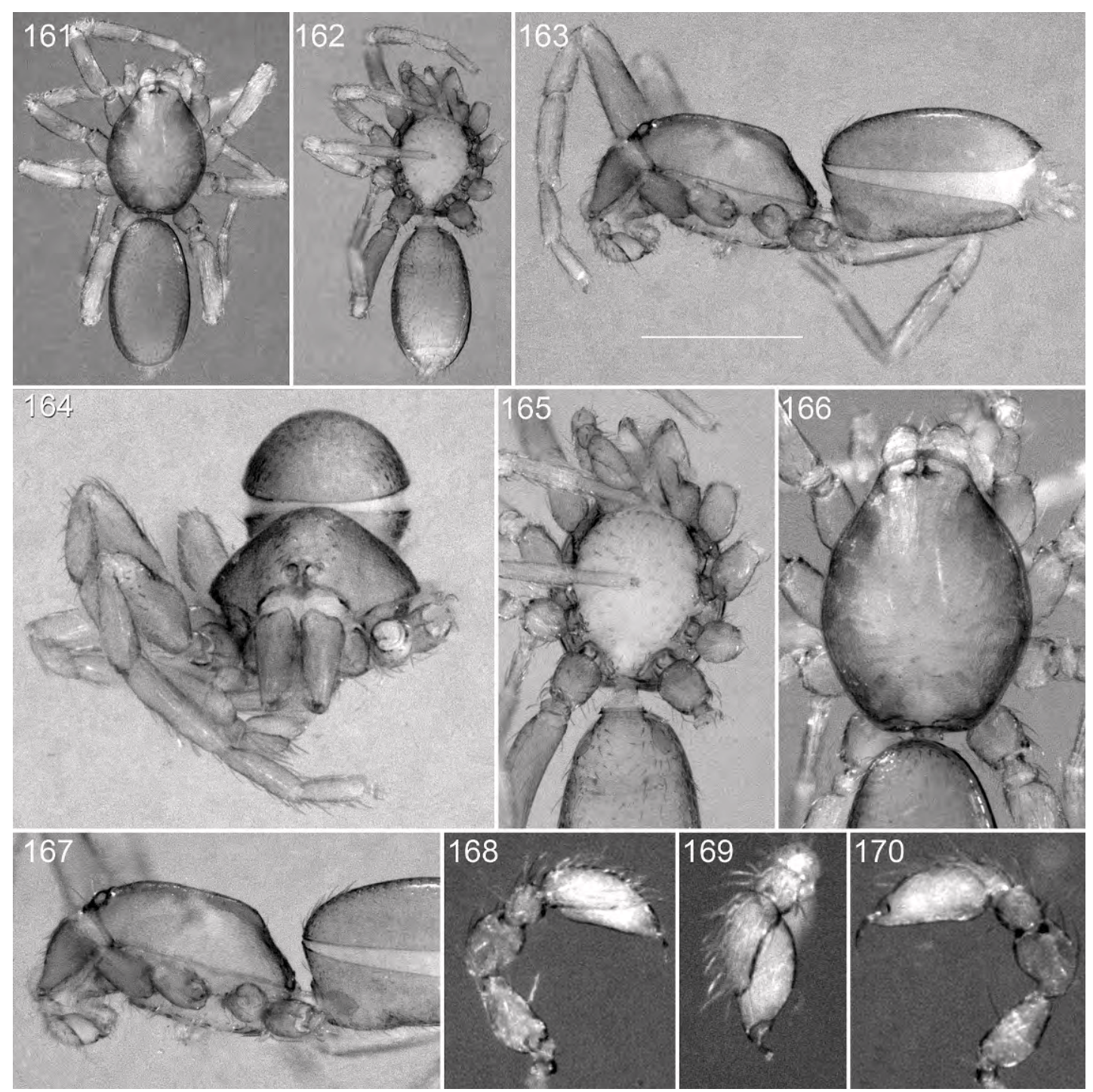

Figs. 161-170. Prethopalpus khasi, n. sp., male (PBI_OON 23387). 161. Habitus, dorsal view. 162. Same, ventral view. 163. Same, lateral view. 164. Prosoma, anterior view. 165. Same, ventral view. 166. Same, dorsal view. 167. Same, lateral view. 168. Male palp, prolateral view. 169. Same, dorsal view 170. Same, retrolateral view.

Prethopalpus brunei Baehr, new species Figures 110-112, 145, 146, 264-282; map 2

TYPE: BRUNEI DARUSSALAM: BruneiMuara District: Male holotype from près du pont sur le ruisseau Sungai Lubang Barus, route venant de Tutong, $33 \mathrm{~km}$ de Bandar Seri Begawan $\left[4.88000^{\circ} \mathrm{N}, 114.93000^{\circ} \mathrm{E}\right], 20 \mathrm{~m}$ (16 Nov. 1988, B. Hauser), deposited in MHNG (PBI_OON 00015305).
ETYMOLOGY: The specific name is a noun in apposition taken from the type locality.

DiAgNosis: Males and females resemble those of $P$. sabah in having a smooth pars cephalica and large eyes, but can be easily recognized by the procurved posterior eye row (figs. 264, 269), males by their much shorter palpal femur (figs. 110, 112), and females by their small round epigynal opening and their long oval internal genital 


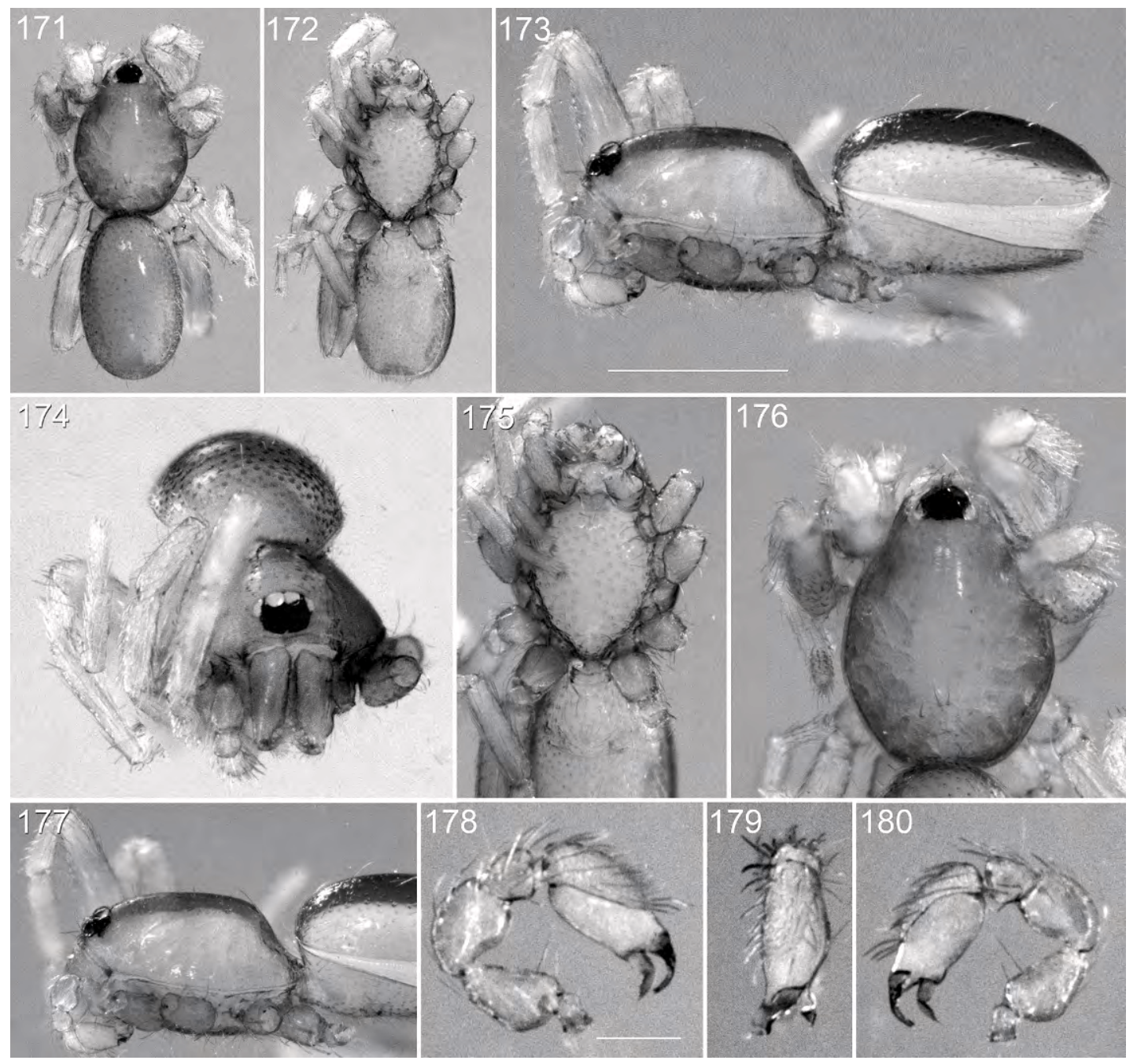

Figs. 171-180. Prethopalpus ilam, n. sp., male (PBI_OON 23390). 171. Habitus, dorsal view. 172. Same, ventral view. 173. Same, lateral view. 174. Prosoma, anterior view. 175. Same, ventral view. 176. Same, dorsal view. 177. Same, lateral view. 178. Male palp, prolateral view. 179. Same, dorsal view 180. Same, retrolateral view.

structure, ending in an anchor-shaped anterior sclerite (figs. 145, 146).

Male (PBI_OON 15305, figs. 264-273): Total length 1.23. Prosoma, mouthparts, and abdominal scuta pale orange, elevated portion of pars cephalica smooth, sides granulate; lateral margin without denticles. Eyes: ALE 0.049; PME 0.042; PLE 0.040; ALE largest, ALE circular, PME squared, PLE circular; posterior eye row procurved from above, straight from front; ALE separated by their radius to diameter, ALE-PLE separated by less than ALE radius, PME touching for less than half their length, PLE-PME touching. Sternum longer than wide, surface finely reticulate, without pits. Endites about 1.5 times as long as wide, with big, blunt toothlike projection. Abdomen ovoid; book lung covers large; pedicel tube short, ribbed. Dorsal scutum covering more than $3 / 4$ of abdomen, smooth; epigastric scutum not protruding; postepigastric scutum long, almost rectangular, covering about $3 / 4$ of abdomen. Legs yellow; patella plus tibia I nearly as long as 


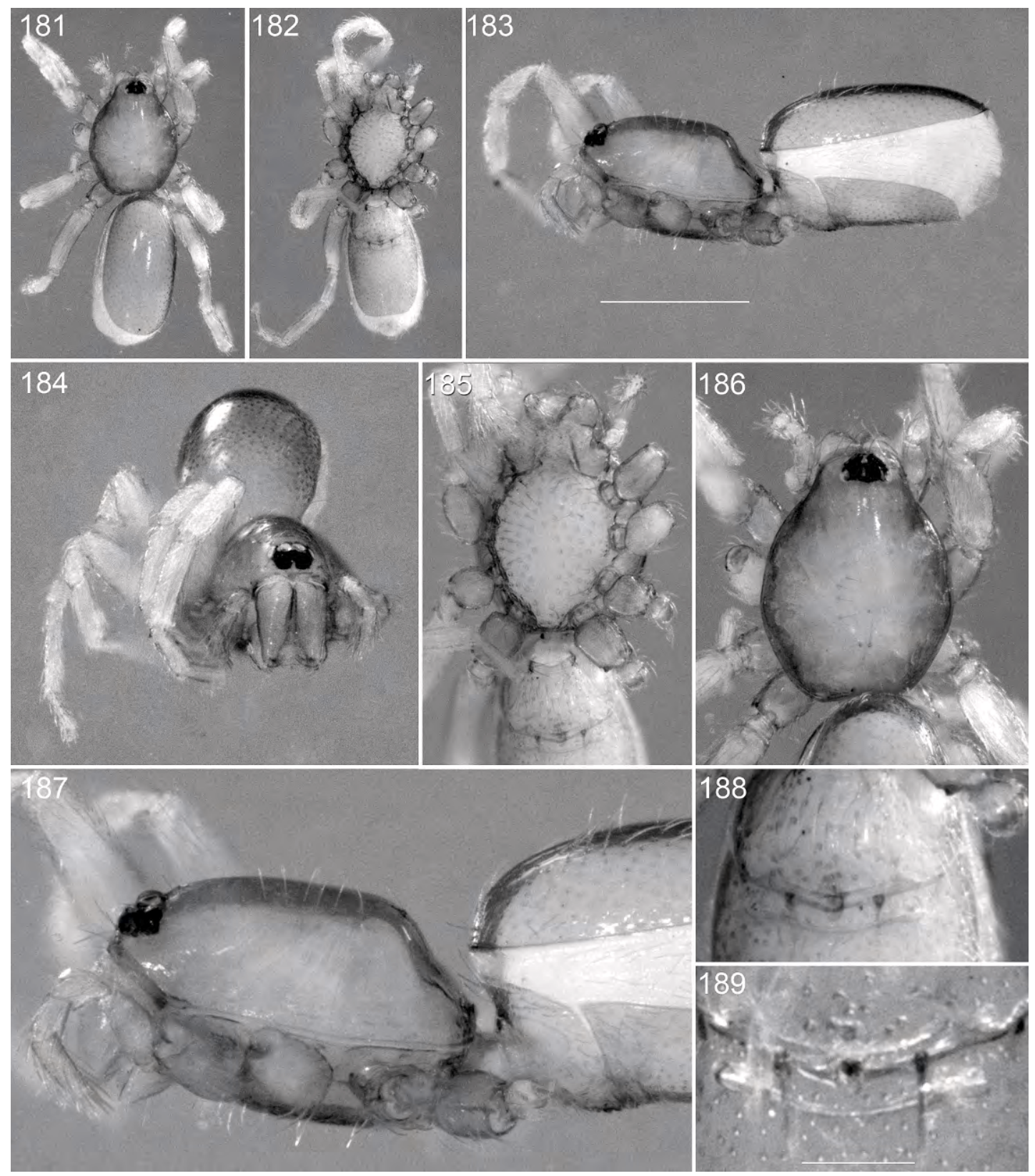

Figs. 181-189. Prethopalpus ilam, n. sp., female (PBI_OON 23391). 181. Habitus, dorsal view. 182. Same, ventral view. 183. Same, lateral view. 184. Prosoma, anterior view. 185. Same, ventral view. 186. Same, dorsal view. 187. Same, lateral view. 188. Female epigyne, ventral view. 189. Same, dorsal view.

carapace. Palp yellow; embolus light; femur attaching to patella subbasally; patella about as long as femur, enlarged; cymbium about as long as bulb, cymbium partly fused with bulb, between cymbium and bulb between four to six dark pits; embolus long thin, bent medially (figs. 110-120).

FemAle (PBI_OON 16225, figs. 274-282). Eyes: ALE 0.047; PME 0.042; PLE 0.036. Epigastric area with small round epigynal 


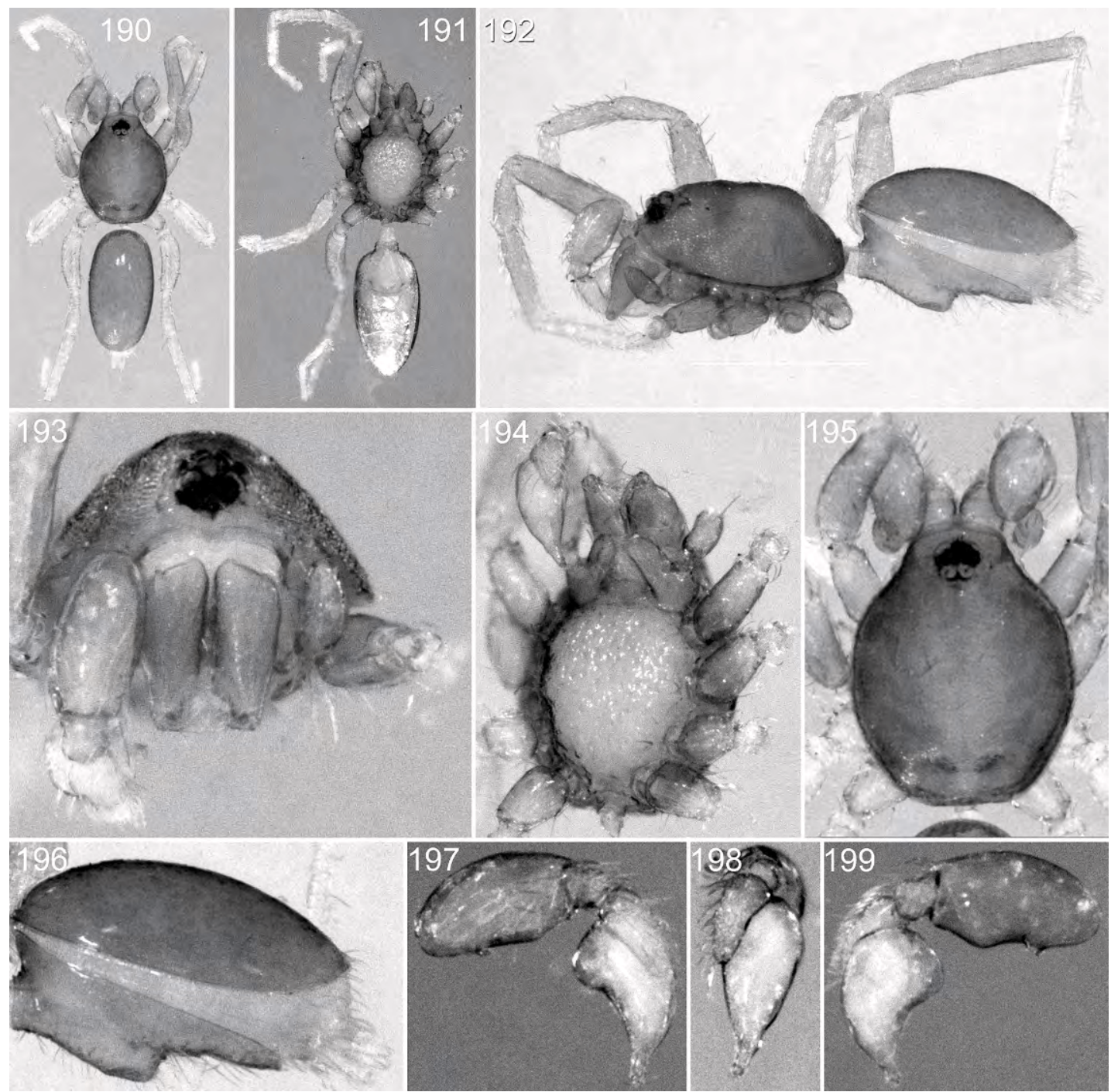

Figs. 190-199. Prethopalpus madurai, n. sp., male (PBI_OON 15598). 190. Habitus, dorsal view. 191. Same, ventral view. 192. Same, lateral view. 193. Prosoma, anterior view. 194. Same, ventral view. 195. Same, dorsal view. 196. Opisthosoma, lateral view. 197. Male palp, prolateral view. 198. Same, dorsal view 199. Same, retrolateral view.

opening, internal genital structure long, oval ending in anchor-shaped anterior sclerite (figs. 145, 146).

OTHER MATERIAL EXAMINED: BRUNEI

\section{DARUSSALAM: Brunei-Muara District:}

Berakas Forest Reserve, N de Nadar Seri Begawan, $19.5 \mathrm{~km}$ de Muara (102.5 de Kuala Belait) $\left[5.17000^{\circ} \mathrm{N}, 115.4000^{\circ} \mathrm{E}\right], 30 \mathrm{~m}, 20$ Nov. 1988, B. Hauser, 1 क, 1 क (MHNG, PBI_OON 00016225). MALAYSIA: Sabah: Kota Kinabalu $\left[6.08333^{\circ} \mathrm{N}, 116.5500^{\circ} \mathrm{E}\right]$,
$1600 \mathrm{~m}, 18$ May 1987, D. Burckhardt and I. Löbl, 1 § (MHNG, PBI_OON 00012312); same location, 16 May 1987, D. Burckhardt and I. Löbl, 1 s , 1 क (MHNG, PBI_OON 00012325); Sarawak: Gunung Matang, $20 \mathrm{~km} \mathrm{W.}$ of Kuching, $1.41666^{\circ} \mathrm{N}, 110.33333^{\circ} \mathrm{E}, 600 \mathrm{~m}, 25$ May 1994, I. Löbl and D. Burckhardt, 1 के (MHNG, PBI_OON 00015248).

DisTRIBUTION: Prethopalpus brunei is known only from Sabah, Sarawak, and Brunei in northern Borneo (map 2). 


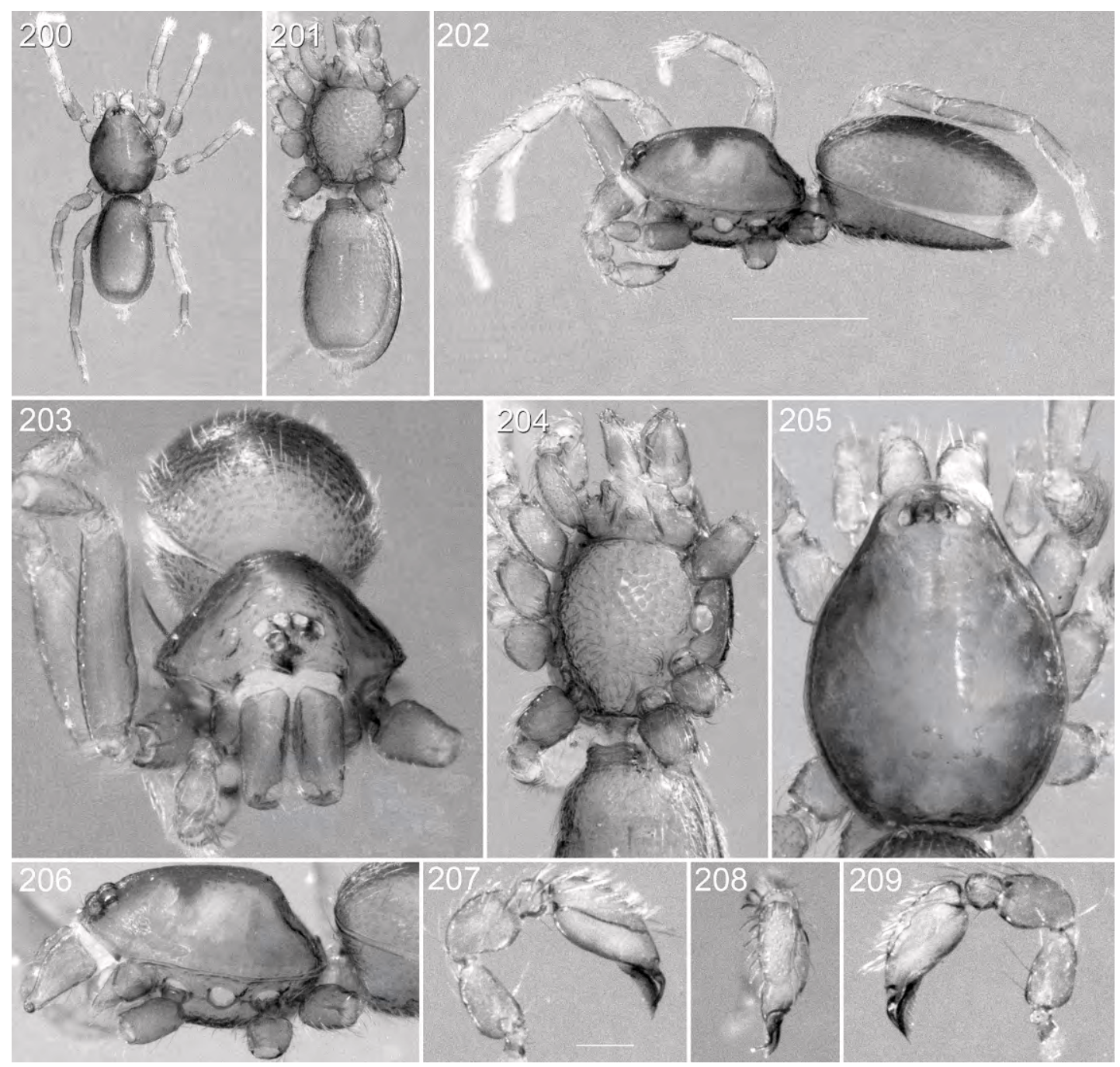

Figs. 200-209. Prethopalpus mahanadi, n. sp., male (PBI_OON 15408). 200. Habitus, dorsal view. 201. Same, ventral view. 202. Same, lateral view. 203. Prosoma, anterior view. 204. Same, ventral view. 205. Same, dorsal view. 206. Same, lateral view. 207. Male palp, prolateral view. 208. Same, dorsal view 209. Same, retrolateral view.

Prethopalpus deelemanae Baehr and Thoma, new species

Figures 283-286; map 5

TYPE: MALAYSIA: Sarawak: Male holotype from Bako National Park, $1.7300^{\circ} \mathrm{N}$, $110.4600^{\circ} \mathrm{E}$ (30 Mar. 1989, C. Deeleman), deposited in RMNH (PBI_OON 00031349).

ETYMOLOGY: This specific name is a patronym in honor of Christa DeelemanReinhold, an extraordinary expert on spiders in the Asian region, who collected the holotype.

DiagNosis: Males resemble those of $P$. brunei in having a smooth pars cephalica and large eyes, but can be easily recognized by the recurved posterior eye row (fig. 283) and the presence of a smooth, median longitudinal band on the sternum (fig. 284).

Male (PBI_OON 31349, figs. 283-286): Total length 1.8. Prosoma mouthparts, and abdominal scuta orange-brown, pars cepha- 


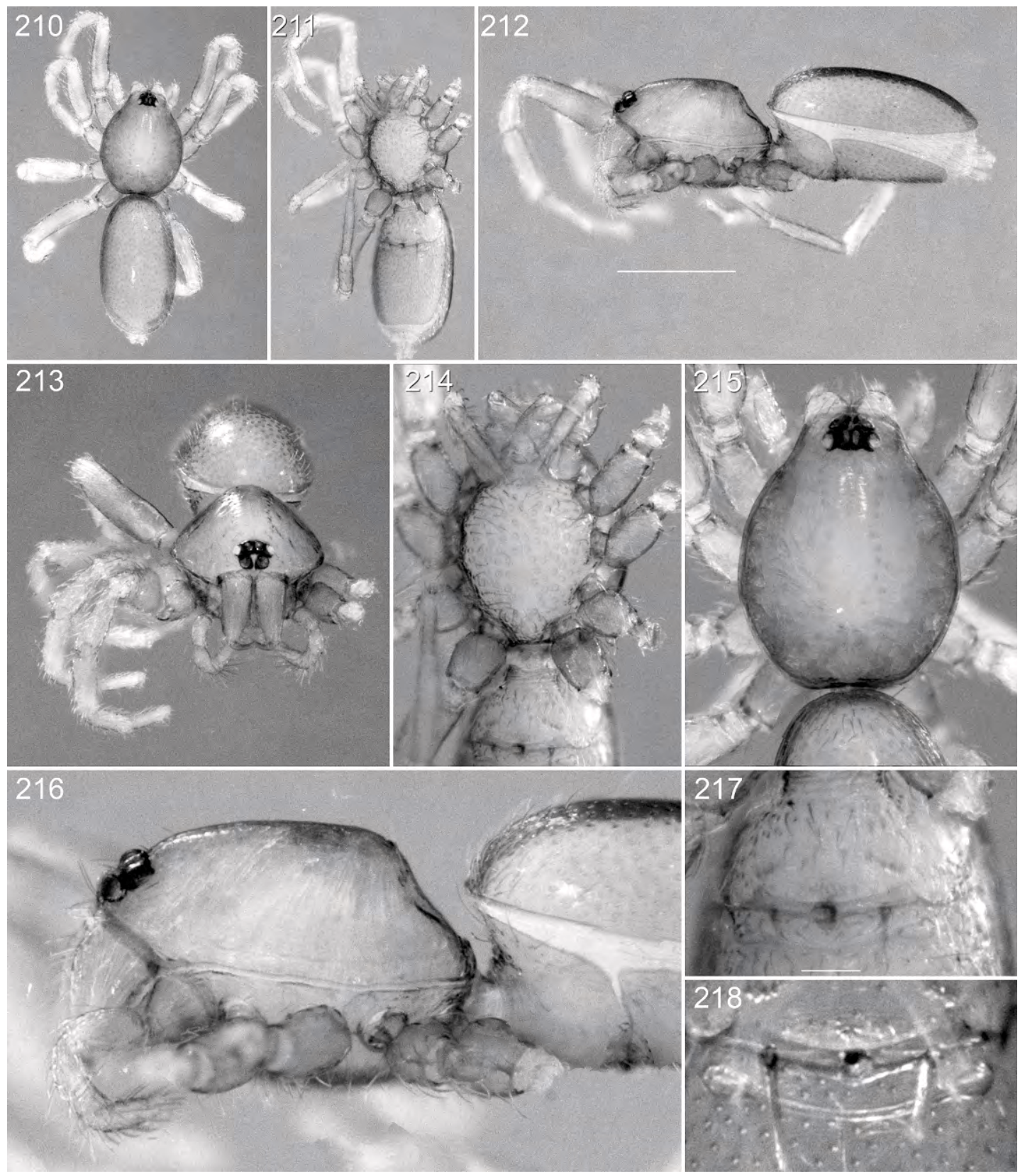

Figs. 210-218. Prethopalpus mahanadi, n. sp., female (PBI_OON 12700). 210. Habitus, dorsal view. 211. Same, ventral view. 212. Same, lateral view. 213. Prosoma, anterior view. 214. Same, ventral view. 215. Same, dorsal view. 216. Same, lateral view. 217. Female epigyne, ventral view. 218. Same, dorsal view.

lica strongly elevated in lateral view, surface smooth; lateral margin with blunt denticles. Clypeus sinuous in front view, vertical in lateral view. Eyes: ALE, PME subequal, larger than PLE, PME oval; posterior eye row recurved from above; ALE separated by their radius to diameter, PLE-PME separated by less than PME radius. Sternum microsculpture everywhere but middle, radial furrows weakly developed; setae evenly 


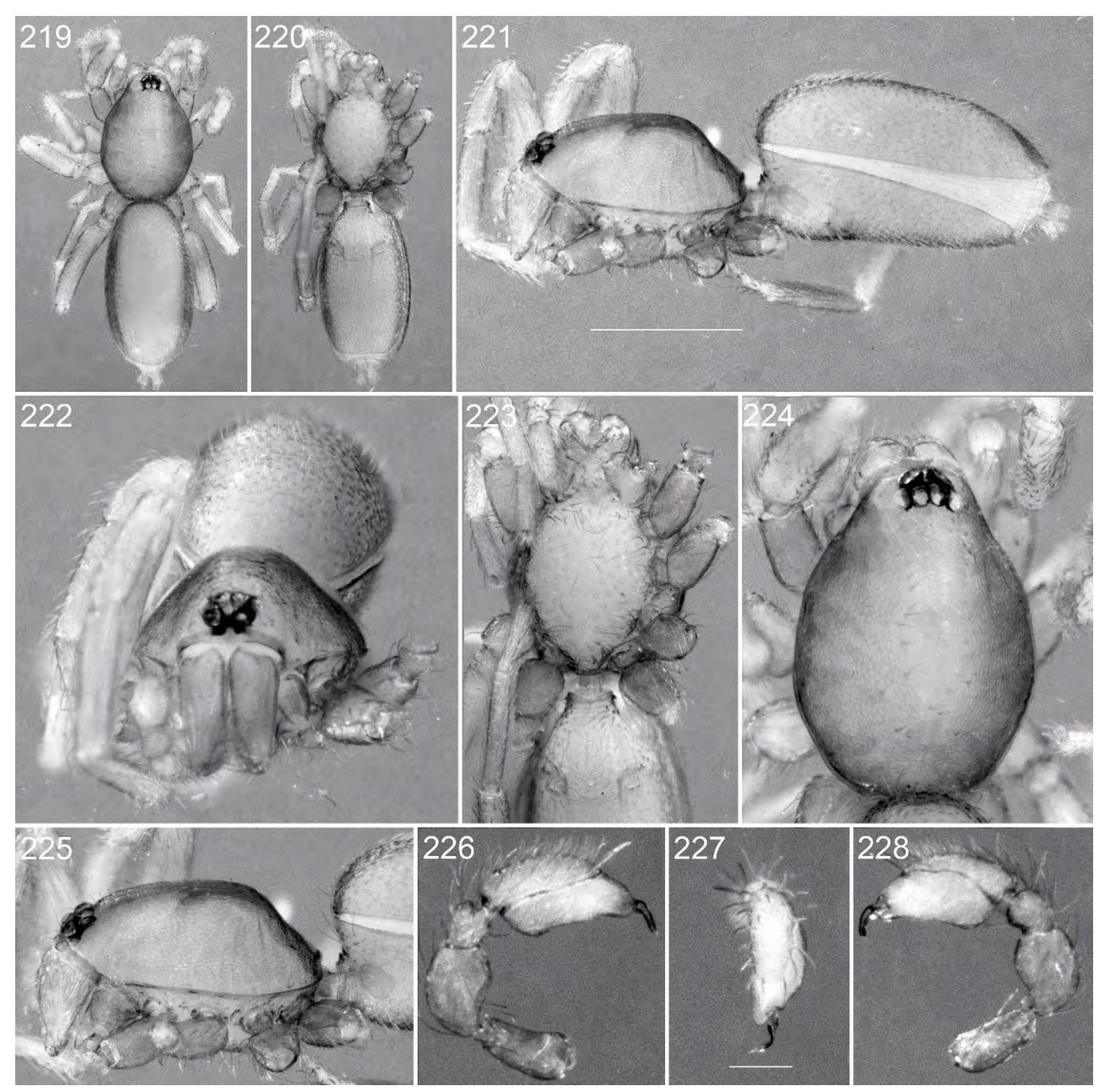

Figs. 219-228. Prethopalpus meghalaya, n. sp., male (PBI_OON 15410). 219. Habitus, dorsal view. 220. Same, ventral view. 221. Same, lateral view. 222. Prosoma, anterior view. 223. Same, ventral view. 224. Same, dorsal view. 225. Same, lateral view. 226. Male palp, prolateral view. 227. Same, dorsal view 228. Same, retrolateral view.

scattered. Labium shape trapezoidal. Abdomen oval; book lung covers round. Dorsal scutum surface smooth, but small pits surrounding hair bases causing punctuate impression; postepigastric scutum almost semicircular. Legs orange-brown, without color pattern. Epigastric region with small circular sperm pore situated between anterior and posterior spiracles. Palp pale orange; embolus dark; femur attaching to patella basally; cymbium partly fused to bulb; between cymbium and bulb with four to six dark pits; embolus long thin, bent medially.

FEMALE: Unknown.

Other Material Examined: None.

Distribution: This species is only known only from Sarawak in eastern Malaysia (map 5). 


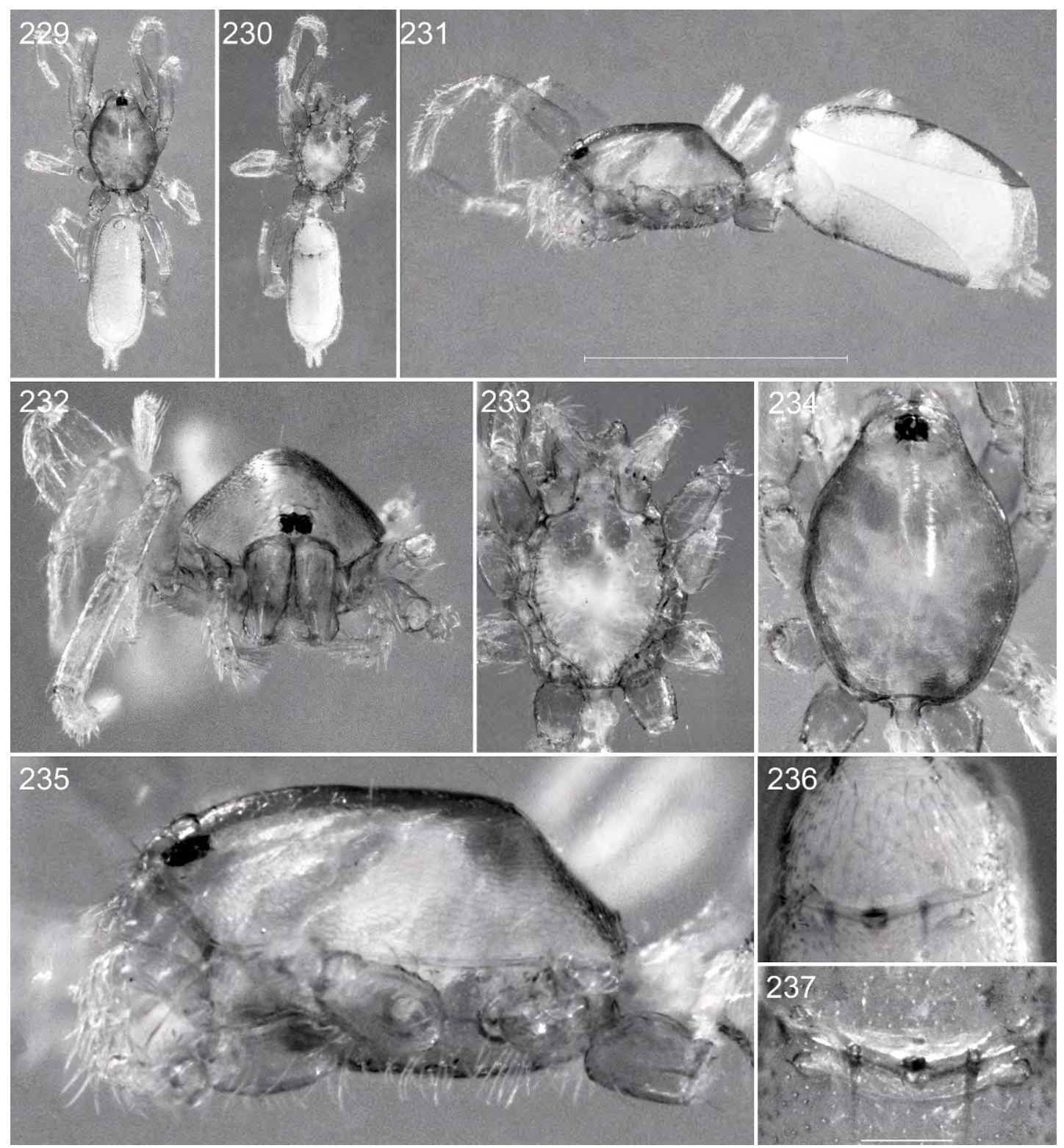

Figs. 229-237. Prethopalpus meghalaya, n. sp., female (PBI_OON 15409). 229. Habitus, dorsal view. 230. Same, ventral view. 231. Same, lateral view. 232. Prosoma, anterior view. 233. Same, ventral view. 234. Same, dorsal view. 235. Same, lateral view. 236. Female epigyne, ventral view. 237. Same, dorsal view.

Prethopalpus fosuma (Burger et al.), new combination

Figures 1-61, 139, 140; map 2

Opopaea fosuma Burger et al., 2002: 244-248, figs. $1-24$.

TYPES: INDONESIA: Sumatera Barat: Male holotype from Kerinci Seblat National
Park, $1.58333^{\circ} \mathrm{S}, 101.3333^{\circ} \mathrm{E}, 800 \mathrm{~m}$ (21-30 July 1988, leaf litter, Suh. Djojosudharmo), deposited in NMB (AR5759, PBI_OON 00023380) examined. Paratypes: collected with holotype, deposited in NMB (AR5760, PBI_OON 00023381), 1 sิ, 2 ㅇ. DiAGNOSIS: Males and females resemble those of $P$. schwendingeri in general body shape and eye size, but can be recognized by 


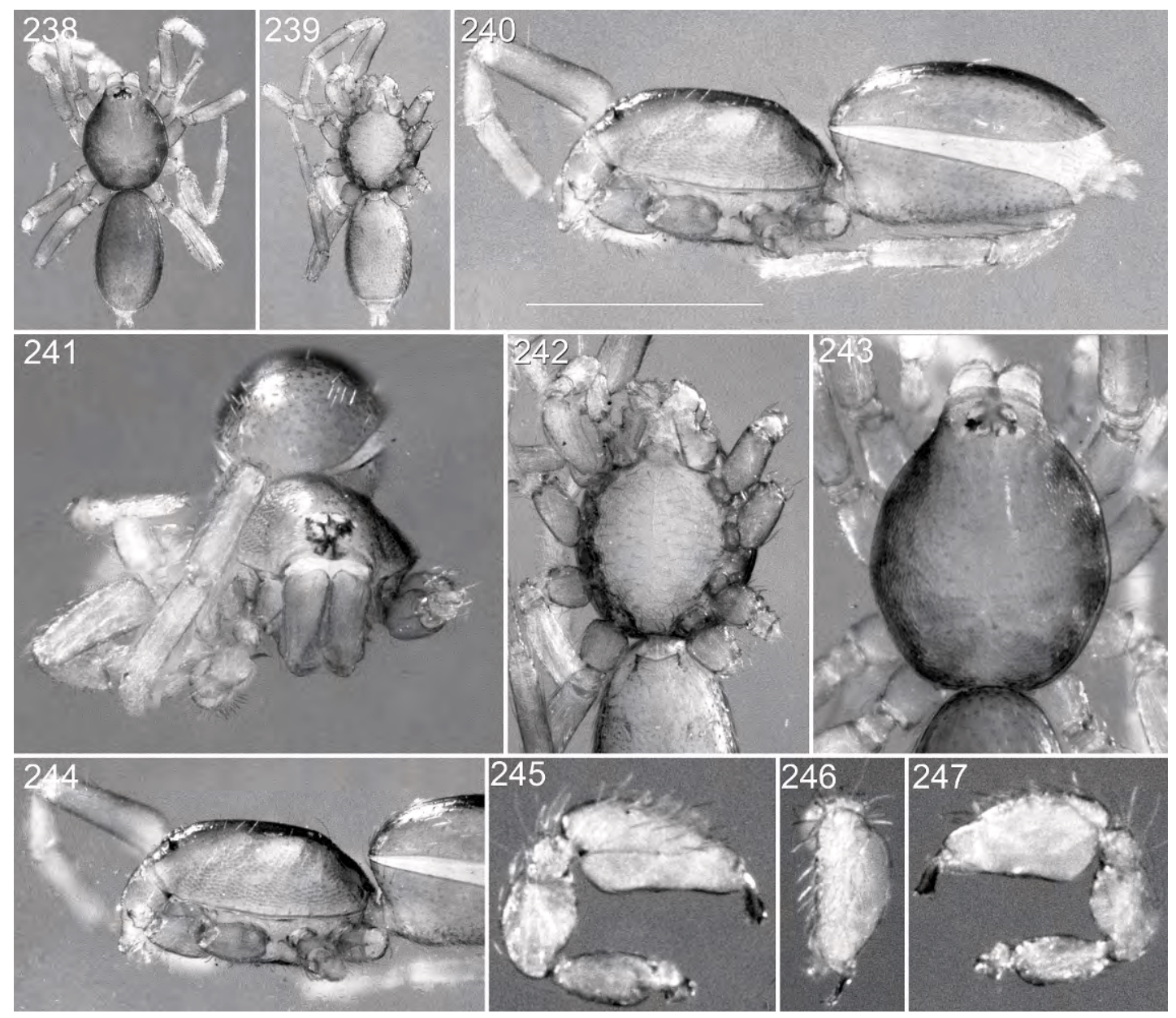

Figs. 238-247. Prethopalpus bali, n. sp., male (PBI_OON 16128). 238. Habitus, dorsal view. 239. Same, ventral view. 240. Same, lateral view. 241. Prosoma, anterior view. 242. Same, ventral view. 243. Same, dorsal view. 244. Same, lateral view. 245. Male palp, prolateral view. 246. Same, dorsal view 247. Same, retrolateral view.

the carapace sides having three recurved granulate patches (figs. 7, 20).

MALE (PBI_OON 16200, figs. 1-10, 1940): Total length 1.25. Prosoma, mouthparts, and scuta orange-brown. Eyes: ALE 0.046; PME 0.041; PLE 0.029; ALE largest, ALE circular, PME oval, PLE circular; posterior eye row recurved from above, straight from front; ALE separated by their radius to diameter, ALE-PLE separated by less than ALE radius, PME touching for less than half their length, PLE-PME separated by less than PME radius. Sternum longer than wide, covered with small round pits, lateral margin with infracoxal grooves and anterior and posterior openings. Endites twice as long as wide anteromedian tip with tiny toothlike projection; book lung covers large; pedicel tube short, ribbed, scutum extending far dorsal of pedicel. Dorsal scutum, covering full length of abdomen, middle surface smooth, sides smooth; epigastric scutum not protruding; postepigastric scutum long, almost rectangular, covering nearly full length of abdomen. Legs pale orange; patella plus tibia I nearly as long as carapace. Superior claws (figs. 34-37) with all surfaces striated; proclaws and retroclaws I-IV each with 8 


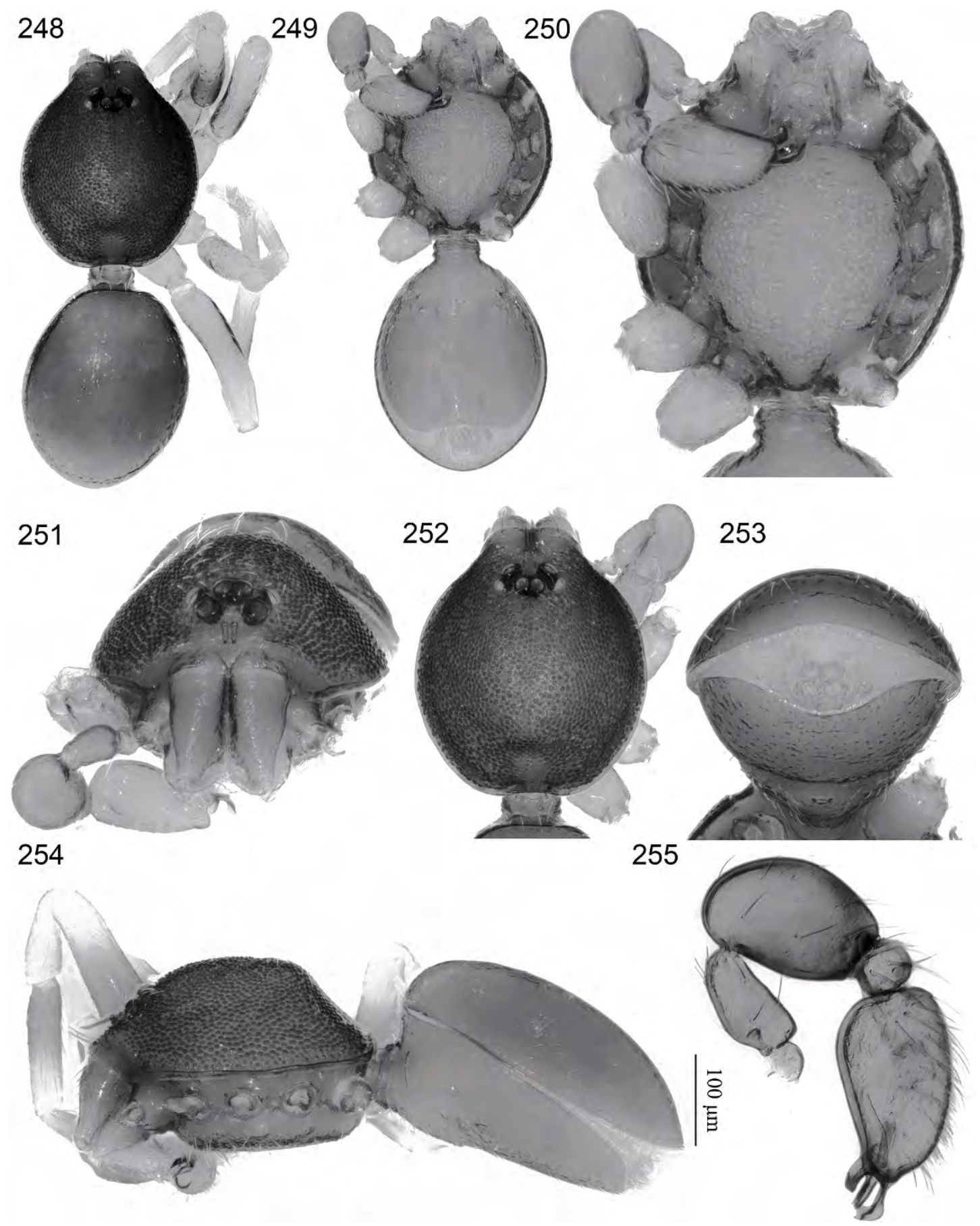

Figs. 248-255. Prethopalpus bellicosus, n. sp., male (PBI_OON 12353). 248. Habitus, dorsal view. 249. Same, ventral view. 250. Prosoma, ventral view. 251. Prosoma, anterior view. 252. Same, dorsal view. 253. Opisthosoma, posterior view. 254. Habitus, lateral view. 255. Male palp, prolateral view. 


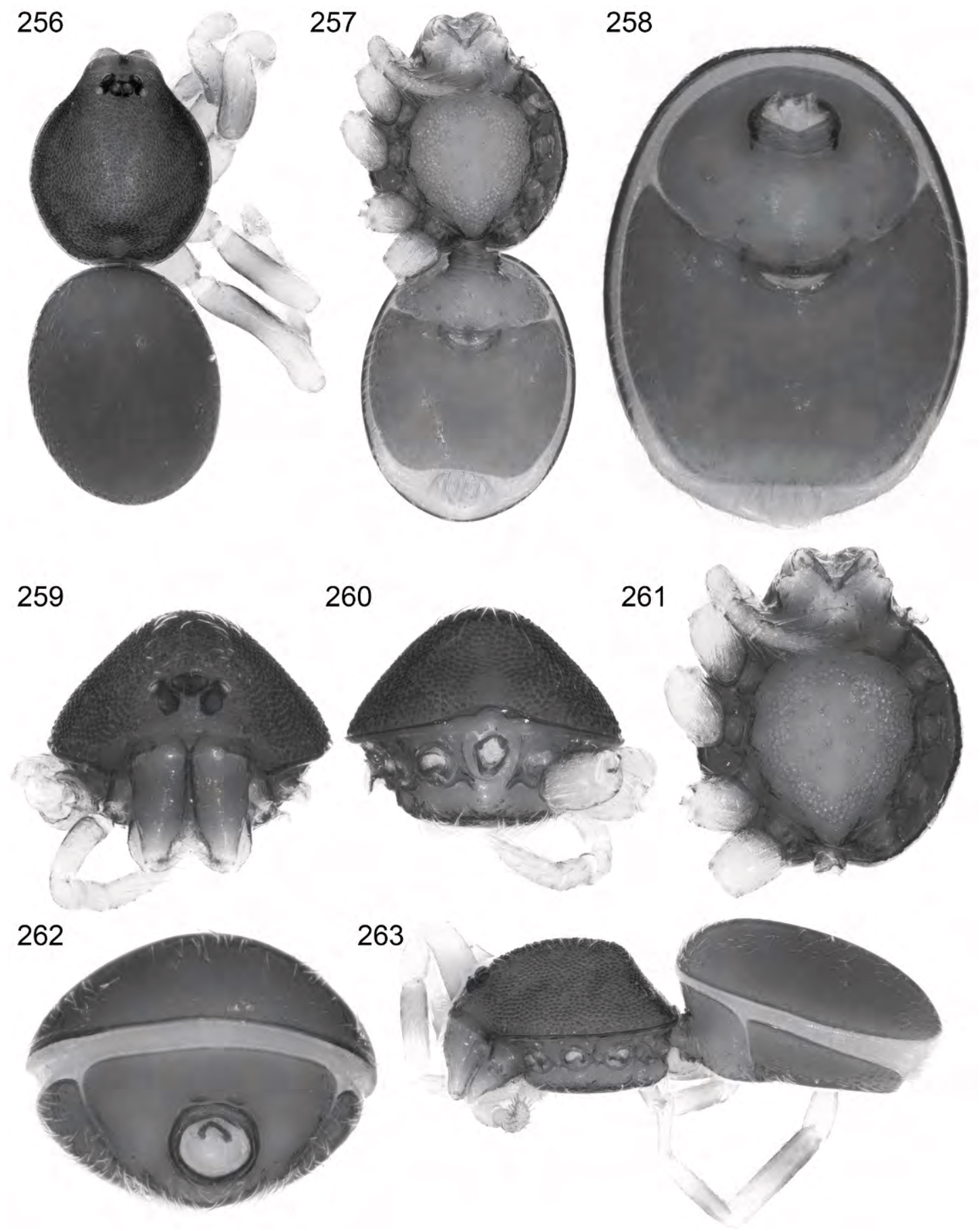

Figs. 256-263. Prethopalpus bellicosus, n. sp., female (PBI_OON 12353). 256. Habitus, dorsal view. 257. Same, ventral view. 258. Opisthosoma, ventral view. 259. Prosoma, anterior view. 260. Same, posterior view. 261. Same, ventral view. 262. Opisthosoma, anterior view. 263. Habitus, lateral view. 
Figs. 264-273. Prethopalpus brunei, n. sp., male (PBI_OON 15305). 264. Habitus, dorsal view. 265. Same, ventral view. 266. Same, lateral view. 267. Prosoma, anterior view. 268. Same, ventral view. 269. Same, dorsal view. 270. Same, lateral view. 271. Male palp, prolateral view. 272. Same, dorsal view 273. Same, retrolateral view.

subapical teeth along inner margin; without inferior claw. Trichobothria (figs. 26-29): tibiae each with three; metatarsus each with one; base longitudinally narrowed, aperture internal texture not gratelike, hood covered by numerous low, closely spaced ridges. Tarsal organ with $2-3$ equal sized sensillae visible (figs. 30-33); 2 sensillae on palpal tarsus (fig. 25). Male genitalia epigastric region with oval, rebordered sperm pore (fig. 23). Palp with proximal segments, cymbium and bulb pale orange; embolus light; femur attaching to patella subbasally; patella about as long as femur; bulb 1 to 1.5 times as long as cymbium, slender, tapering apically, distal part with retrolateral impression and long, thin, medially bent embolus (figs. 38-40).

Female (PBI_OON 12527, figs. 1118, 41-61): Total length 1.97. Eyes: ALE 0.085; PME 0.080; PLE 0.077. Dorsal scutum not fused to epigastric scutum. Legs: trichobothria (figs. 48-51); tarsal organ (figs. 5255). Copulatory opening oval (fig. 139), leading into central, elongated receptaculum; short, mushroomlike gland ducts on either side of receptaculum (figs. 58-61); anterior sclerite T-shaped (figs. 57, 59); apodemes strong. 


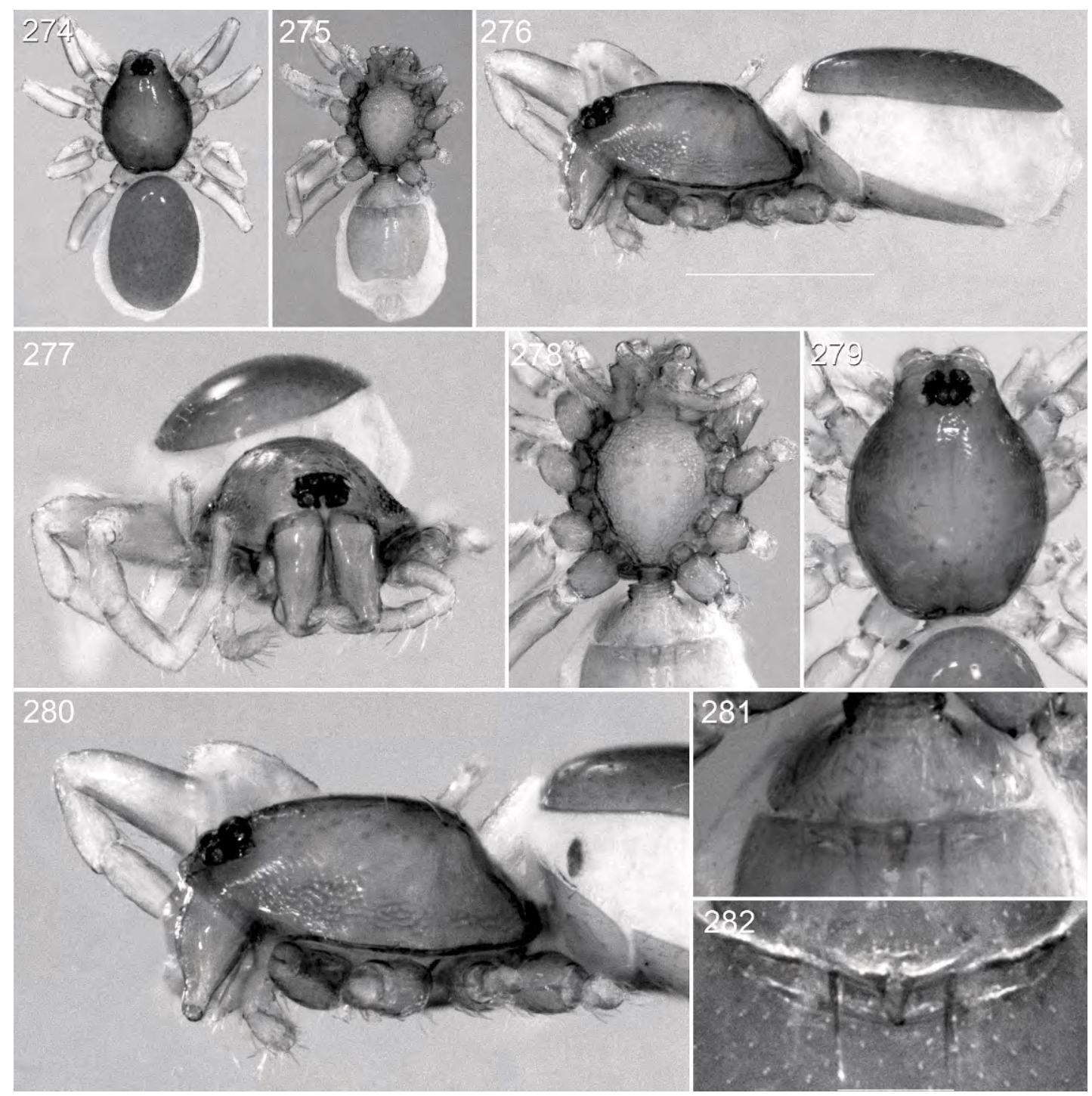

Figs. 274-282. Prethopalpus brunei, n. sp., female (PBI_OON 16225). 274. Habitus, dorsal view. 275. Same, ventral view. 276. Same, lateral view. 277. Prosoma, anterior view. 278. Same, ventral view. 279. Same, dorsal view. 280. Same, lateral view. 281. Female epigyne, ventral view. 282. Female epigyne, dorsal view.

Other MATERIAl ExAmined: INDONESIA: Jawa Barat: Bandung, $30 \mathrm{~km} \mathrm{~N}$, Volcan Tangkuban Prahu, au pied du volcan, $6.86666^{\circ} \mathrm{S}, 107.66666^{\circ} \mathrm{E}, 7$ Aug. 1984 , J. Robert, 1 ㅇ (MHNG, PBI_OON 00012502); Bogor, Jardin Botanique $\left[6.56660^{\circ} \mathrm{S}, 106.75000^{\circ} \mathrm{E}\right]$, 250 m, 24 Nov. 1987, B. Hauser, 2 के (MHNG, PBI_OON 00016200); Col du Puncak et Cibodas, reserve de Cibodas $\left[7.33333^{\circ} \mathrm{S}\right.$, $\left.106.61670^{\circ} \mathrm{E}\right], 4$ Aug. 1984 , J. Robert, 3 के (MHNG, PBI_OON 00012599); Gunung Gede-Pangrango National Park near Cibodas, $6.7833^{\circ} \mathrm{S}, 107.0166^{\circ} \mathrm{E}, 1450 \mathrm{~m}, 4-11$ May 2005, A. Schulz, 1 (MHNG, PBI_OON 00015621). Sumatera Barat: Kerinci Seblat National Park, $1.58333^{\circ} \mathrm{S}, 101.33333^{\circ} \mathrm{E}$, 800 m, 21-30 July 1988, Suh. Djojosudharmo, $1 \hat{\sigma}, 2$ + (NMB AR5760, PBI_OON 


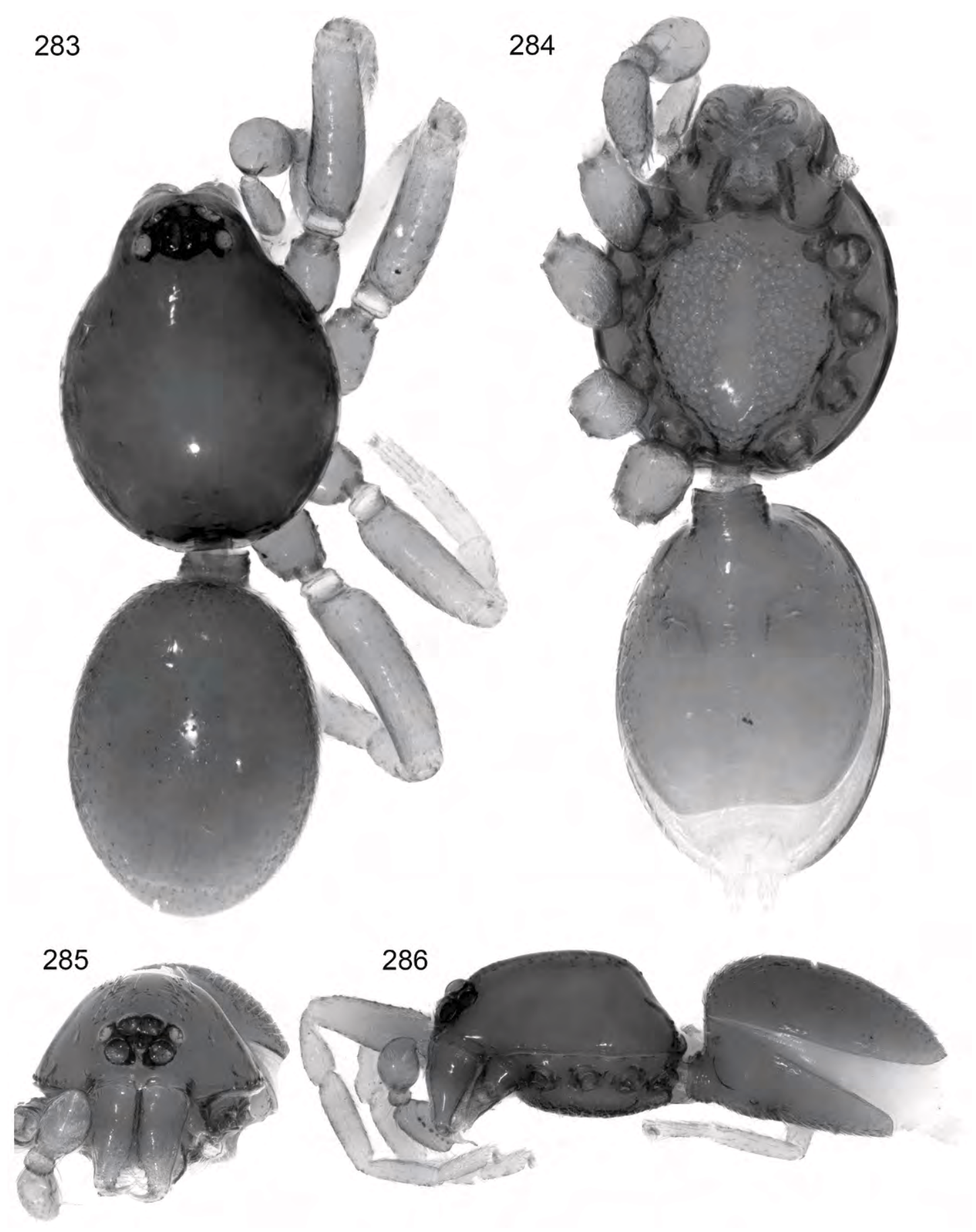

Figs. 283-286. Prethopalpus deelemanae, n. sp., male (PBI_OON 31349). 283. Habitus, dorsal view. 284. Same, ventral view. 285. Prosoma, anterior view. 286. Habitus, lateral view. 


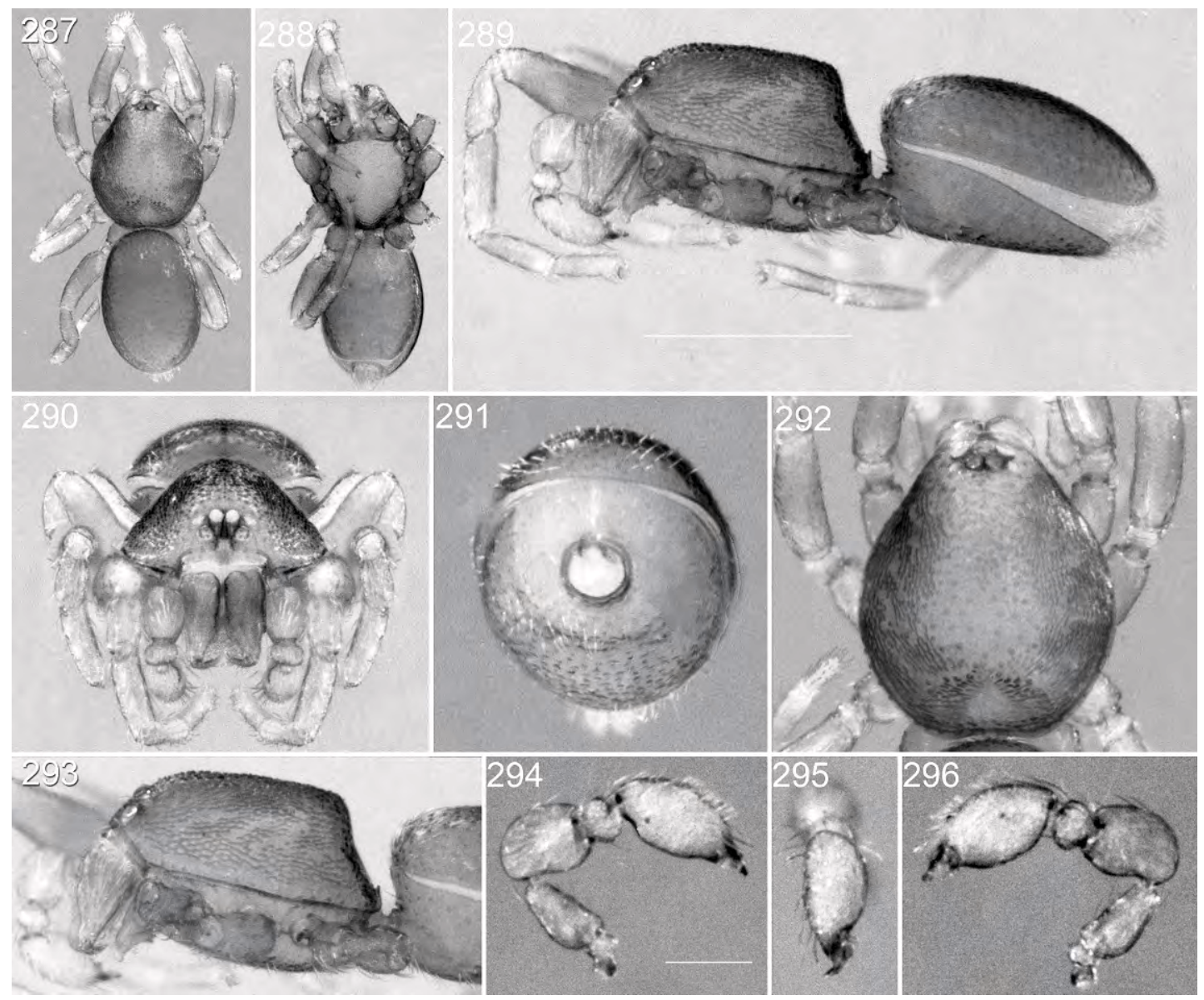

Figs. 287-296. Prethopalpus java, n. sp., male (PBI_OON 12086). 287. Habitus, dorsal view. 288. Same, ventral view. 289. Same, lateral view. 290. Prosoma, anterior view. 291. Opisthosoma, anterior view. 292. Prosoma, dorsal view. 293. Same, lateral view. 294. Male palp, prolateral view. 295. Same, dorsal view 296. Same, retrolateral view.

00023381); Mt Kerinci, Jambi $\left[1.55000^{\circ} \mathrm{S}\right.$, 101.20000 ${ }^{\circ} \mathrm{E}$, $1900 \mathrm{~m}, 13$ June 1989, I. Löbl, D. Agosti and D. Burckhardt, 1 of (MHNG, PBI_OON 00012087); Padangpanjan, $10 \mathrm{~km}$ W $\left[0.27578^{\circ} \mathrm{S}, 100.22494^{\circ} \mathrm{E}\right], 250 \mathrm{~m}, 17$ Nov. 1989, I. Löbl, D. Agosti and D. Burckhardt, 1 ș, 1 \& (MHNG, PBI_OON 00012079). Sumatera Bengkulu: Taba Penanjung Reserve, road Taba Penan jung-Kepahiang $\left[3.80044^{\circ} \mathrm{S}, 102.26554^{\circ} \mathrm{E}\right], 700 \mathrm{~m}, 27 \mathrm{Feb}$. 2000, P. Schwendinger, 3 s, 5 क (MHNG, PBI_OON 00012813); ca. $20 \mathrm{~km}$ SE of Bengkulu City, Hutan Raya (Botanical Garden) Bengkulu, $3.8000^{\circ} \mathrm{N}, 102.2666^{\circ} \mathrm{E}, 50 \mathrm{~m}$, 29 Feb. 2000, P. Schwendinger, 7 s, 3 ㅇ (MHNG, PBI_OON 00012377).
Distribution: Prethopalpus fosuma is known from several locations in Sumatra and Java, Indonesia (map 2).

Prethopalpus java Baehr, new species Figures 101-103, 147, 148, 287-305; map 2

TYPE: INDONESIA: Jawa Barat: Male holotype from Cibodas $\left[6.85000^{\circ} \mathrm{S}\right.$, $\left.107.28333^{\circ} \mathrm{E}\right], 50 \mathrm{~km} \mathrm{E}$ of Bogor, $1400 \mathrm{~m}$ (3-6 Nov. 1989, I. Löbl, D. Agosti and D. Burckhardt), deposited in MHNG (PBI OON 00012086). Paratype: Cibodas, Jardin Botanique, $7.31666^{\circ} \mathrm{S}, 106.61666^{\circ} \mathrm{E}, 1380 \mathrm{~m}$ (25 Nov. 1987, B. Hauser), deposited in MHNG (PBI_OON 00016211), 1 ㅇ. 


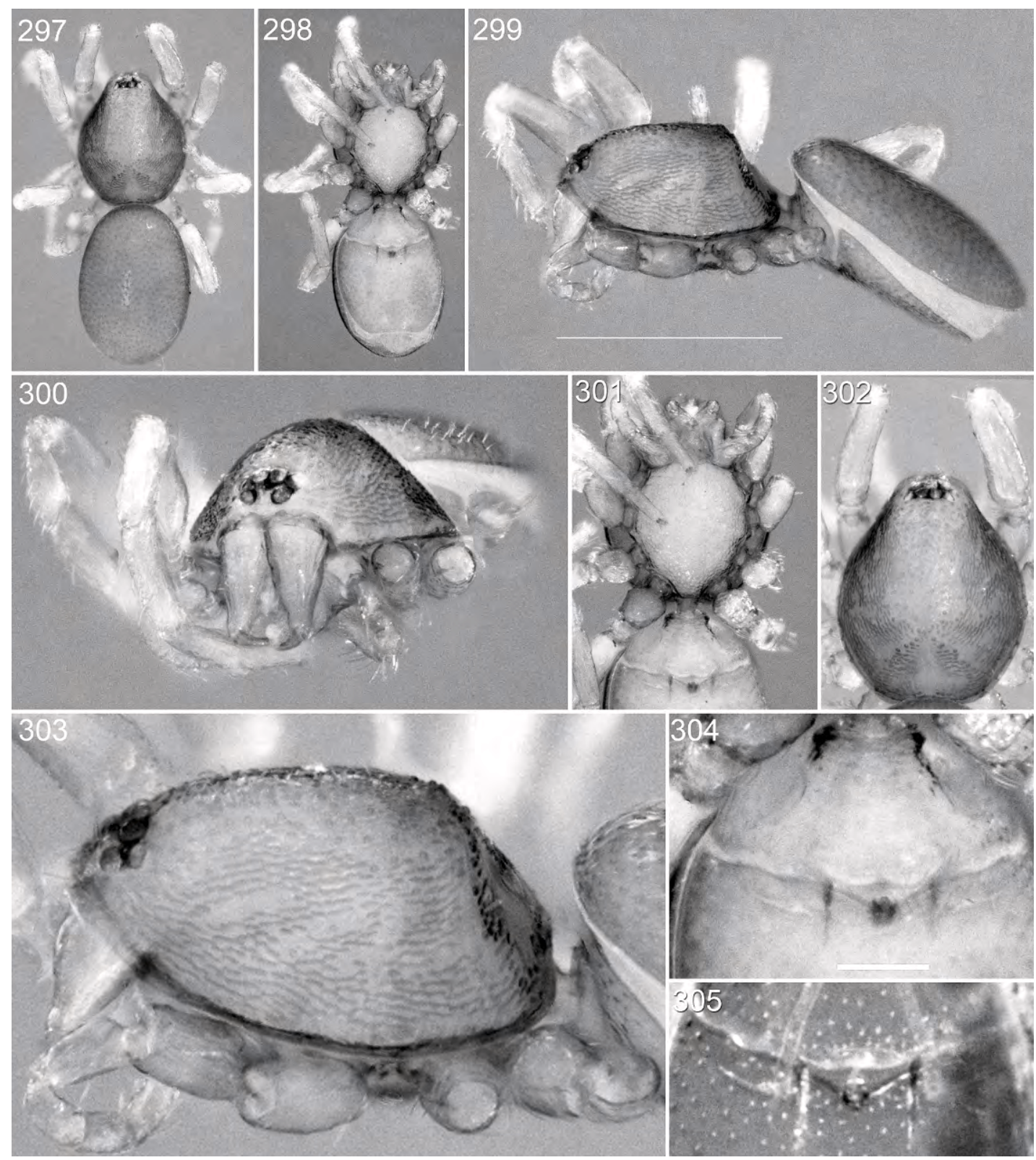

Figs. 297-305. Prethopalpus java, n. sp., female (PBI_OON 16211). 297. Habitus, dorsal view. 298. Same, ventral view. 299. Same, lateral view. 300. Prosoma, anterior view. 301. Same, ventral view. 302. Same, dorsal view. 303. Same, lateral view. 304. Female epigyne, ventral view. 305. Female epigyne, dorsal view.

ETYMOLOGY: The specific name is a noun in apposition taken from the type locality.

DiAgnosis: Males and females resemble those of $P$. kropfi in having a strongly granulated carapace, but can be easily recognized by the smaller eyes (figs. 287,
292), the recurved posterior eye row (figs. 287, 292), and the reticulated sternal area without a smooth median part (fig. 288).

MALE (PBI_OON 12086, figs. 287-296): Total length 0.97 . Prosoma, mouthparts, and abdominal scuta pale orange, pars cephalica 


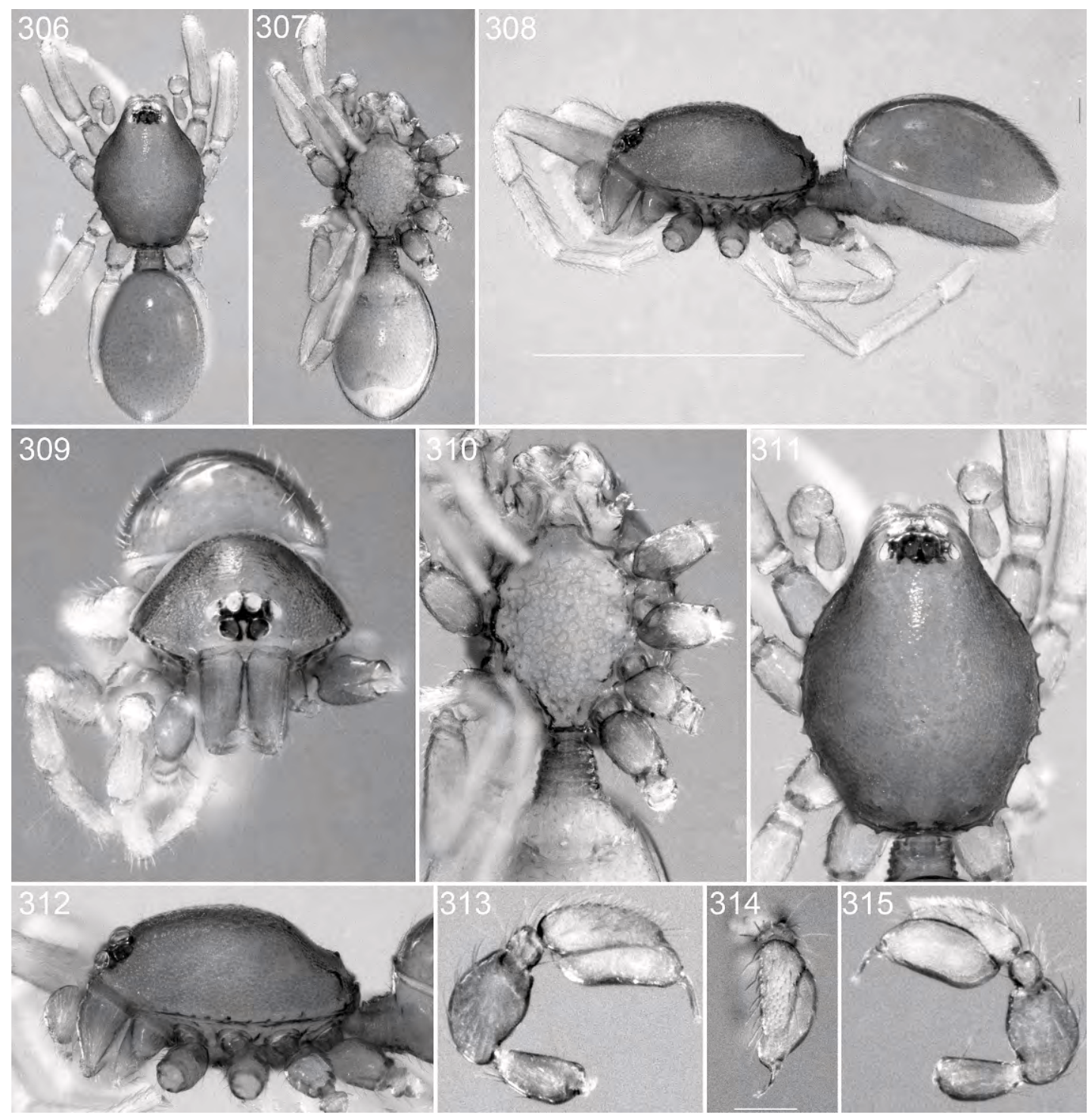

Figs. 306-315. Prethopalpus kranzae, n. sp., male (PBI_OON 12082). 306. Habitus, dorsal view. 307. Same, ventral view. 308. Same, lateral view. 309. Prosoma, anterior view. 310. Same, ventral view. 311. Same, dorsal view. 312. Same, lateral view. 313. Male palp, prolateral view. 314. Same, dorsal view 315. Same, retrolateral view.

strongly elevated in lateral view, elevated portion of pars cephalica and sides strongly granulate; lateral margin without denticles. Eyes small, ALE 0.037; PME 0.030; PLE 0.028; ALE largest, ALE circular, PME circular, PLE oval; posterior eye row recurved from above, straight from front; ALE separated by more than their diameter, ALEPLE separated by less than ALE radius,
PME separated by less than their radius, PLE-PME separated by less than PME radius. Sternum longer than wide, surface finely reticulate, without pits. Endites about as long as wide; anteromedian toothlike projection short. Abdomen ovoid; book lung covers large; pedicel tube short, ribbed. Dorsal scutum covering full length of abdomen smooth; epigastric scutum not 


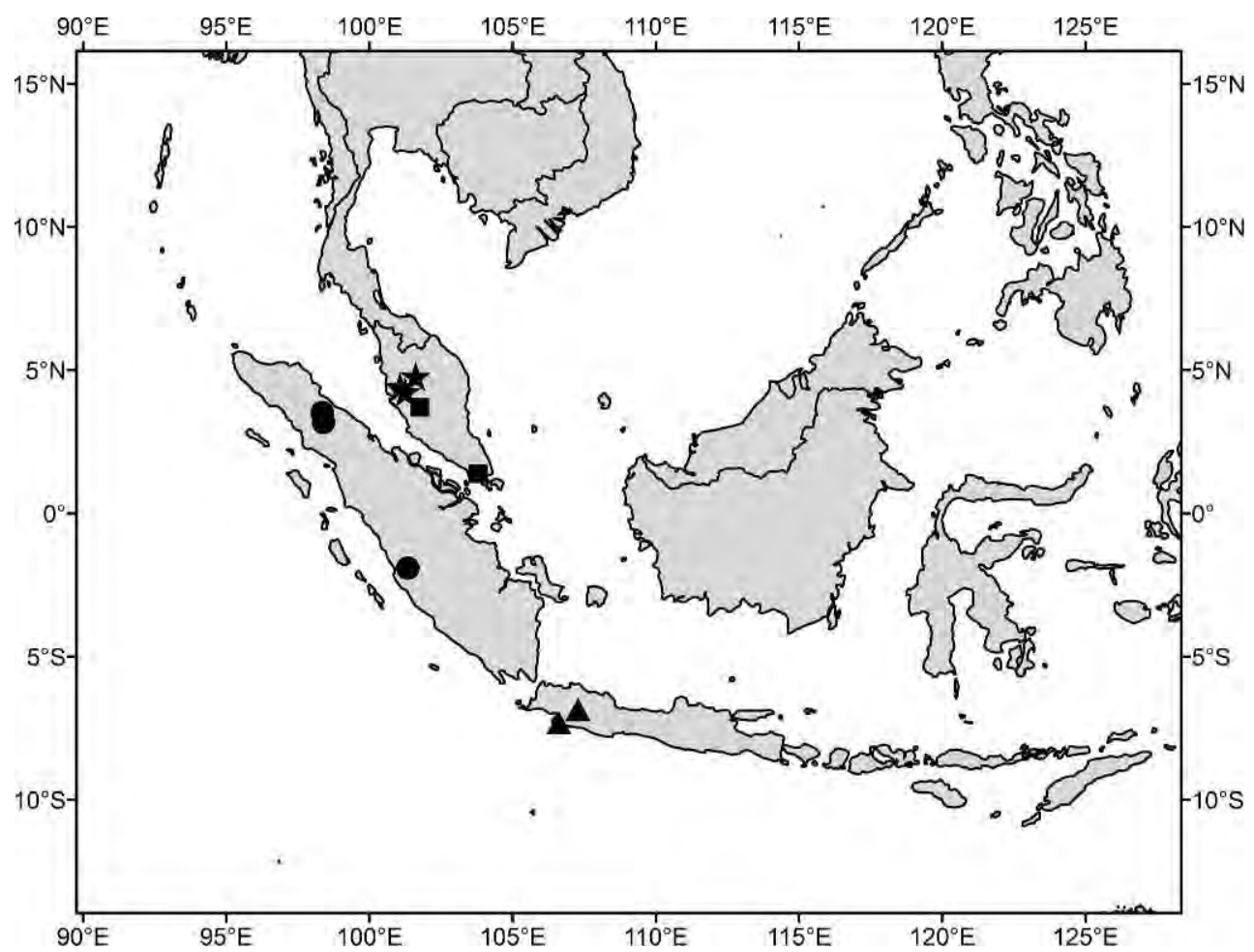

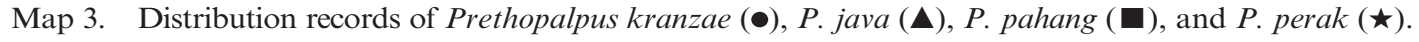

protruding; postepigastric scutum long, almost rectangular, covering nearly full length of abdomen. Legs pale orange; patella plus tibia I shorter than carapace. Palp yellow; embolus light; femur attaching to patella subbasally; patella about as long as femur, enlarged; bulb only slightly separated from cymbium by 1 pit, pyriform with large retrolateral excavation distally, and short triangular medially bent embolus (figs. 101103).

Female (PBI_OON 00016211, figs. 297305): Total length 1.8. Eyes: ALE 0.036; PME 0.032; PLE 0.030. Epigastric area with small round epigynal opening, internal genital structure pentagonal ending in an anchor-shaped anterior sclerite (figs. 147, 148)

OTHER MATERIAL EXAMINED: INDONESIA: Jawa Barat: Cibodas $\left[6.85000^{\circ} \mathrm{S}\right.$, $\left.107.28333^{\circ} \mathrm{E}\right], 50 \mathrm{~km} \mathrm{E}$ of Bogor, $1400 \mathrm{~m}, 3-6$ Nov. 1989, I. Löbl, D. Agosti, and D. Burckhardt, 1 s (MHNG, PBI_OON 00012086).

Distribution: Prethopalpus java is known only from Java (map 2).
Prethopalpus kranzae Baehr, new species Figures 83-85, 306-315; map 3

TYPE: INDONESIA: Aceh: Male holotype from Gunung Leuser National Park, Ketambe [3.17193 $\left.\mathrm{N}, 98.39077^{\circ} \mathrm{E}\right], 300 \mathrm{~m}$ (23-30 Nov. 1989, I. Löbl, D. Agosti, and D. Burckhardt), deposited in MHNG (PBI_OON 00012082).

ETYMOLOGY: The specific name is a patronym in honor of Yvonne Kranz-Baltensperger of the Natural History Museum, Bern.

DiAgNosis: Males can be separated from those of all other species by the posterolateral surface of the carapace bearing one pair of spikes (fig. 312), the lateral carapace margin with sharply pointed denticles (fig. 311), and the long pedicel tube (fig. 310).

MALE (PBI_OON 12082, figs. 306-315): Total length 1.65. Prosoma, mouthparts, and abdominal scuta yellow-brown, posterolateral surface with one pair of spikes, carapace, strongly granulated; lateral margin with sharply pointed denticles. Eyes: ALE 0.063; PME 0.055; PLE 0.053; ALE largest, ALE 


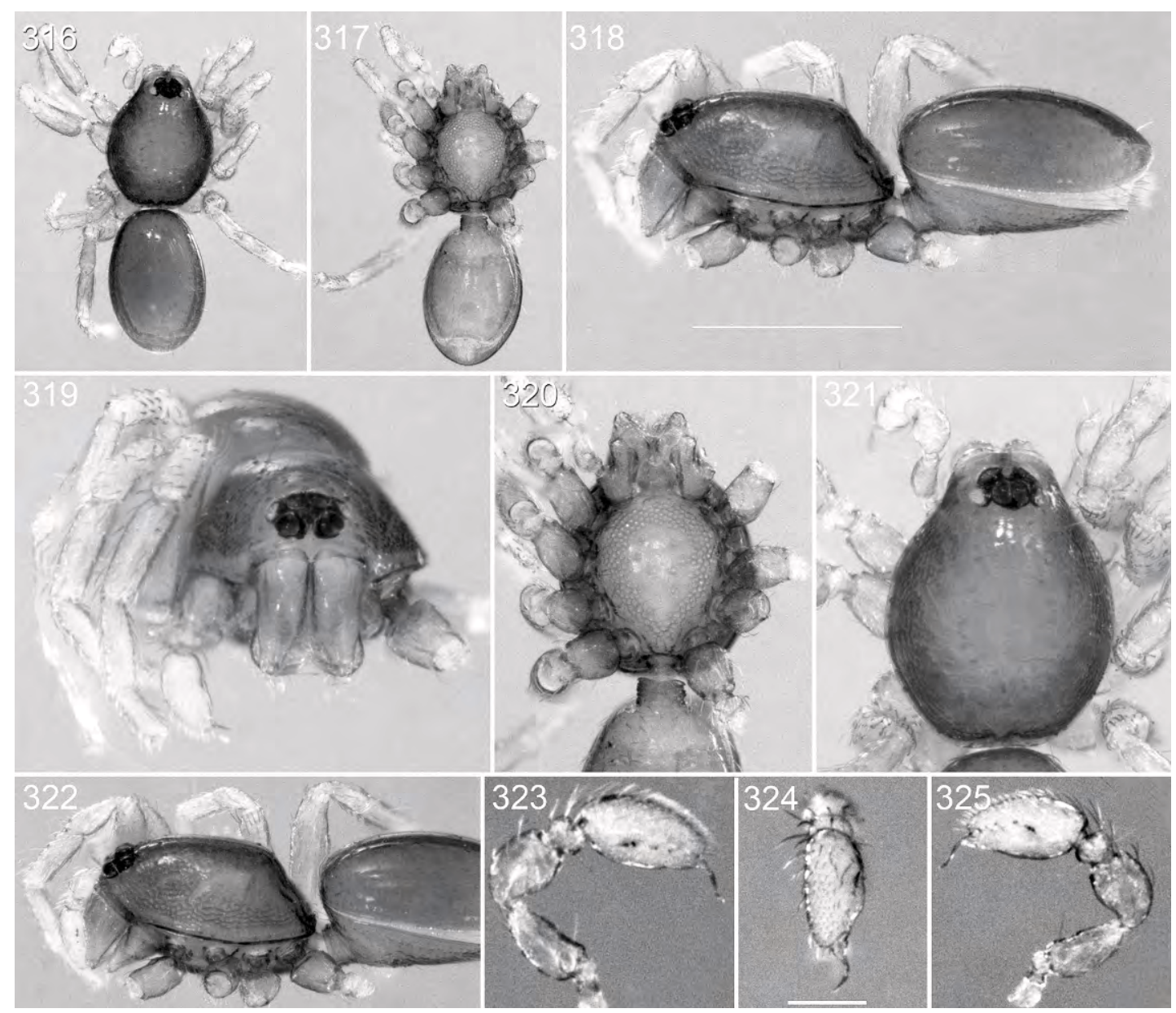

Figs. 316-325. Prethopalpus kropfi, n. sp., male (PBI_OON 12741). 316. Habitus, dorsal view. 317. Same, ventral view. 318. Same, lateral view. 319. Prosoma, anterior view. 320. Same, ventral view. 321. Same, dorsal view. 322. Same, lateral view. 323. Male palp, prolateral view. 324. Same, dorsal view 325. Same, retrolateral view.

circular, PME oval, PLE circular; posterior eye row straight from above and front; ALE separated by their radius to diameter, ALEPLE separated by ALE radius to ALE diameter, PME touching for less than half their length, PLE-PME separated by less than PME radius. Sternum wider than long, surface rugose, covered with three pairs of large round pits laterally. Anteromedian tip of endites unmodified, twice as long as wide. Abdomen ovoid; book lung covers large; pedicel tube long, ribbed. Dorsal scutum covering full length of abdomen, smooth; epigastric scutum not protruding; postepigastric scutum long, almost rectangular, covering nearly full length of abdomen, with long posteriorly directed lateral apodemes. Legs yellow-brown; patella plus tibia I shorter than carapace. Epigastric region with oval sperm pore. Palp yellow-brown; embolus light; femur attaching to patella subbasally; patella about as long as femur, enlarged; cymbium not fused with bulb, bulb pyriform, apical tip with deep incision and slightly medially bent embolus (figs. 8385).

FEMALE: Unknown.

Other Material Examined: None.

Distribution: This species is known only from Sumatra (map 3). 


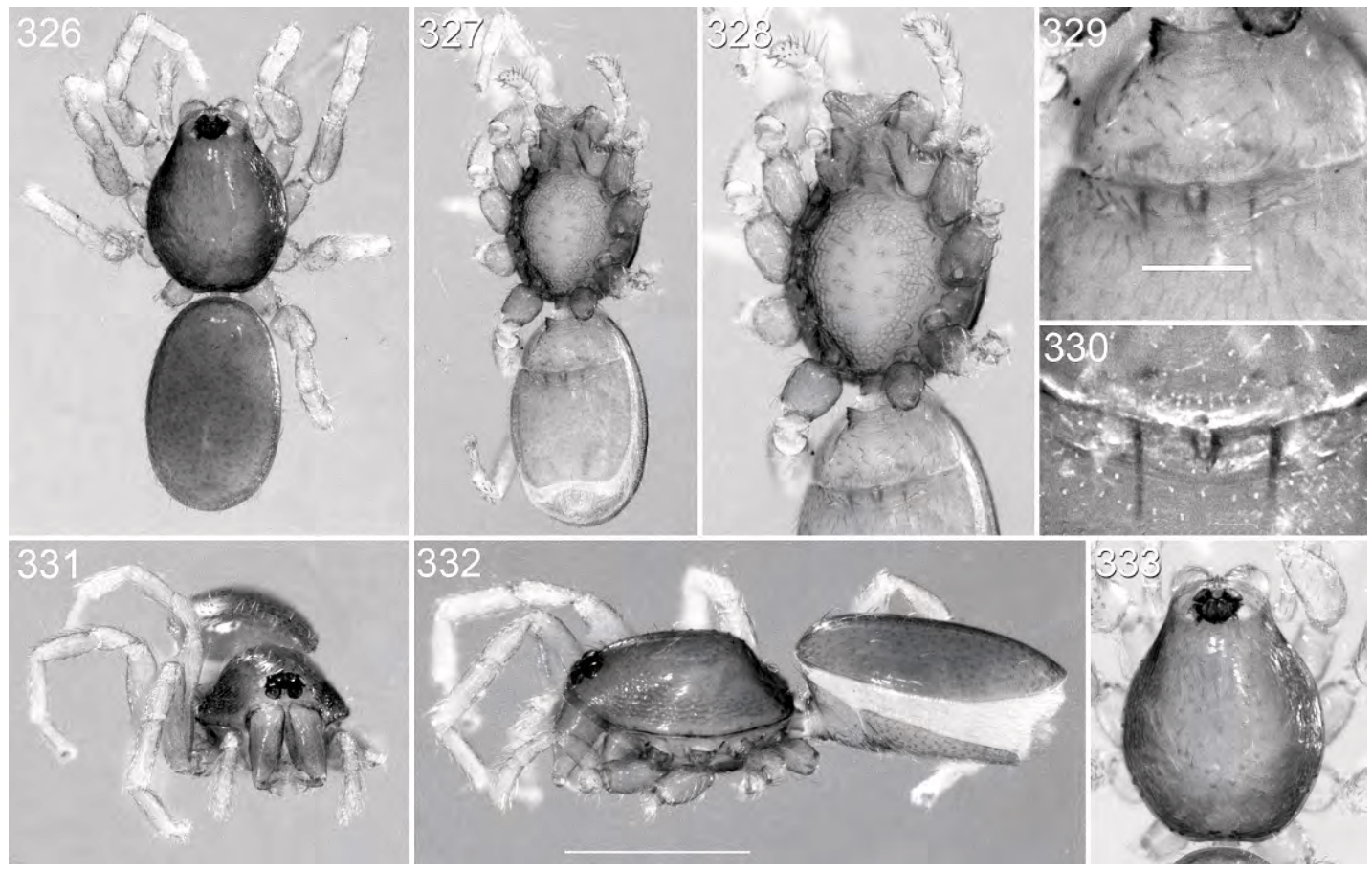

Figs. 326-333. Prethopalpus kropfi, n. sp., female (PBI_OON 23394). 326. Habitus, dorsal view. 327. Same, ventral view. 328. Prosoma, ventral view. 329. Female epigyne, ventral view. 330. Female epigyne, dorsal view. 331. Prosoma, anterior view. 332. Habitus, lateral view. 333. Prosoma, dorsal view.

Prethopalpus kropfi Baehr, new species Figures 104-106, 153, 154, 316-339; map 5

TyPes: MALAYSIA: Sarawak: Male holotype from Mulu National Park, $4^{\circ} 0000 \mathrm{~N}$, $114.8166^{\circ} \mathrm{E}, 200 \mathrm{~m}$ (19-24 Aug. 2003, A. Schulz), deposited in MHNG (PBI_OON 00012741). Female allotype collected with holotype, deposited in MHNG (PBI_OON 00023394).

ETYMOLOGY: The specific name is a patronym in honor of Christian Kropf of the Natural History Museum, Bern.

Diagnosis: Males and females resemble those of $P$. java in having a strongly granulated carapace, but can be easily recognized by the larger eyes (fig. 316), the straight posterior eye row (fig. 316), and the reticulated sternal area with a smooth median part (fig. 320).

MALE (PBI_OON 12741, figs. 316-325): Total length 1.18. Prosoma, mouthparts, and abdominal scuta yellow-brown, surface of elevated portion of pars cephalica smooth, sides with continuous rough striation front higher; lateral margin with blunt denticles. Eyes: ALE 0.050; PME 0.046; PLE 0.042; ALE largest, ALE circular, PME squared, PLE circular; posterior eye row straight from both above and front; ALE separated by less than their radius, ALE-PLE separated by less than ALE radius, PME touching throughout most of their length, PLE-PME separated by less than PME radius. Sternum longer than wide, surface with inverted teardrop-shaped smooth median area and granulate margin, smooth median area covered with setae originating from few deep pits; radial furrows very short and weakly developed. Endites twice as long as wide, with tiny toothlike projection. Abdomen ovoid; book lung covers large; pedicel tube short, ribbed. Dorsal scutum covering full length of abdomen, smooth; epigastric scutum not protruding; postepigastric scutum long, almost rectangular, covering nearly full length of abdomen. Legs yellow-brown; patella plus tibia I shorter than carapace. Palp pale 


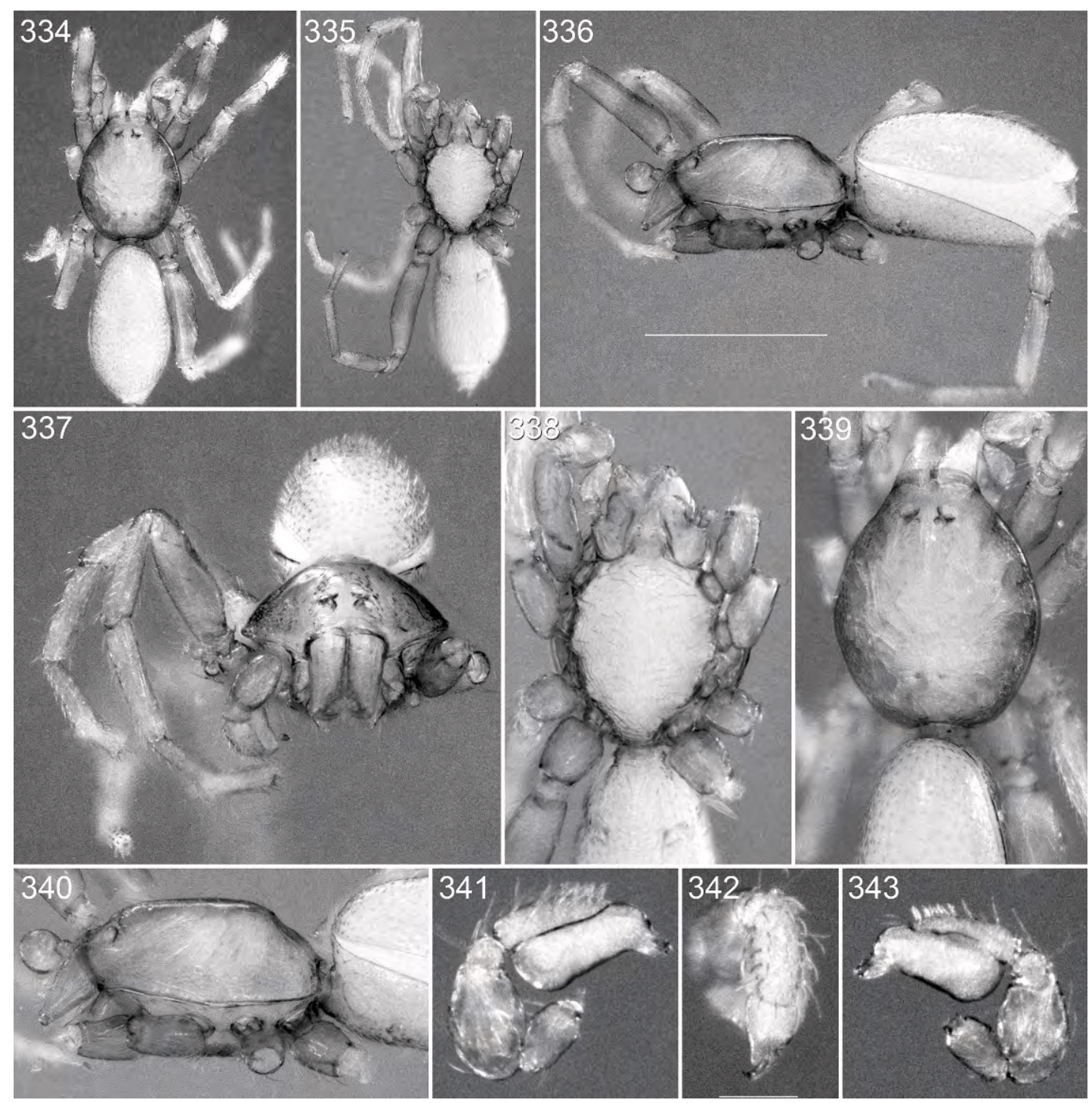

Figs. 334-343. Prethopalpus leuser, n. sp., male (PBI_OON 12083). 334. Habitus, dorsal view. 335. Same, ventral view. 336. Same, lateral view. 337. Prosoma, anterior view. 338. Same, ventral view. 339. Same, dorsal view. 340. Same, lateral view. 341. Male palp, prolateral view. 342. Same, dorsal view. 343. Same, retrolateral view.

orange; embolus light; femur attaching to patella subbasally; patella as long as femur, enlarged; cymbium and bulb hardly separated with about five stronger sclerotized pits proand retrolaterally along the separation line; bulbal tip with retrolateral impression and thin, medially bent embolus (figs. 104-106).

Female (PBI_OON 23394, figs. 326333): Total length 1.29. Eyes: ALE 0.045;
PME 0.049; PLE 0.041. Epigastric area with small oval epigynal opening, long oval, internal genital region almost reaching to posterior groove, ending in almost triangular anterior sclerite (figs. 153, 154).

Other Material Examined: MALAYSIA: Kedah: Pulau Langkawi, Gunung Raya, NE side, $6.4000^{\circ} \mathrm{N}, 99.8333^{\circ} \mathrm{E}, 400 \mathrm{~m}, 29-30$ Dec. 2004, A. Schulz, 2 i (MHNG, 


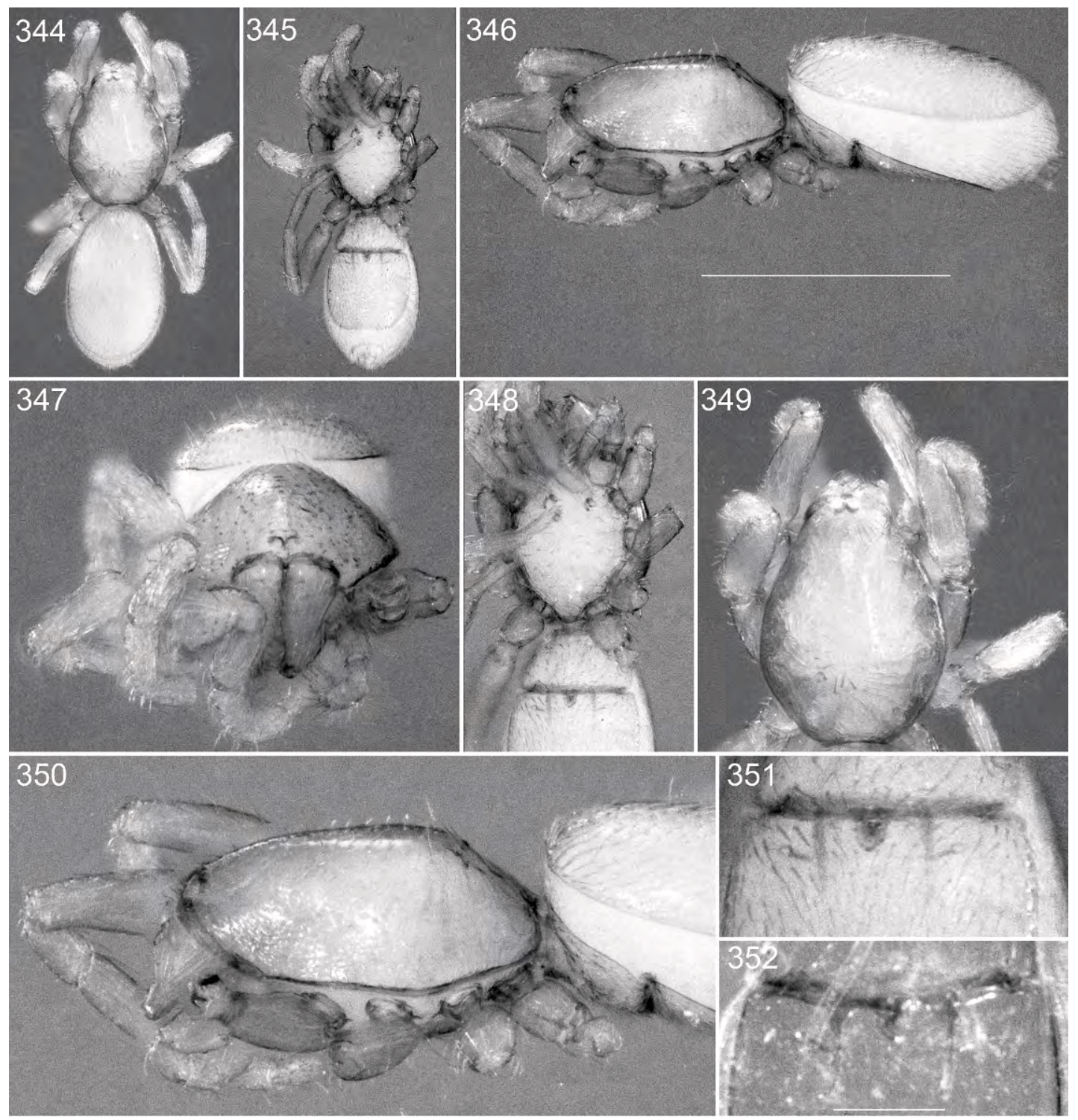

Figs. 344-352. Prethopalpus leuser, n. sp., female (PBI_OON 23397). 344. Habitus, dorsal view. 345. Same, ventral view. 346. Same, lateral view. 347. Prosoma, anterior view. 348. Same, ventral view. 349. Same, dorsal view. 350. Same, lateral view. 351. Female epigyne, ventral view. 352. Female epigyne, dorsal view.

PBI_OON 00012115). Sarawak: Mulu National Park, $4.0000^{\circ} \mathrm{N}, 114.81666^{\circ} \mathrm{E}, 200 \mathrm{~m}$, 19-24 Aug. 2003, A. Schulz, 1 ㅇ (MHNG, PBI_OON 00023395). Sabah: Crocker Range, km 63 route Kota Kinabalu-Tambunan, $1200 \mathrm{~m}, 5.66600^{\circ} \mathrm{N}, 116.33330^{\circ} \mathrm{E}, 19$ May 1987, D. Burckhardt and I. Löbl, $1 \hat{\sigma}$
(MHNG, PBI_OON 00012276); Kibongol Valley, $7 \mathrm{~km} \mathrm{~N}$ of Tambunan, $700 \mathrm{~m}$, $5.66600^{\circ} \mathrm{N}, 116.00000^{\circ} \mathrm{E}, 20$ May 1987, D. Burckhardt and I. Löbl, 1 s (MHNG, PBI_OON 00012181).

Distribution: Prethopalpus kropfi is known from Kedah State on peninsular 


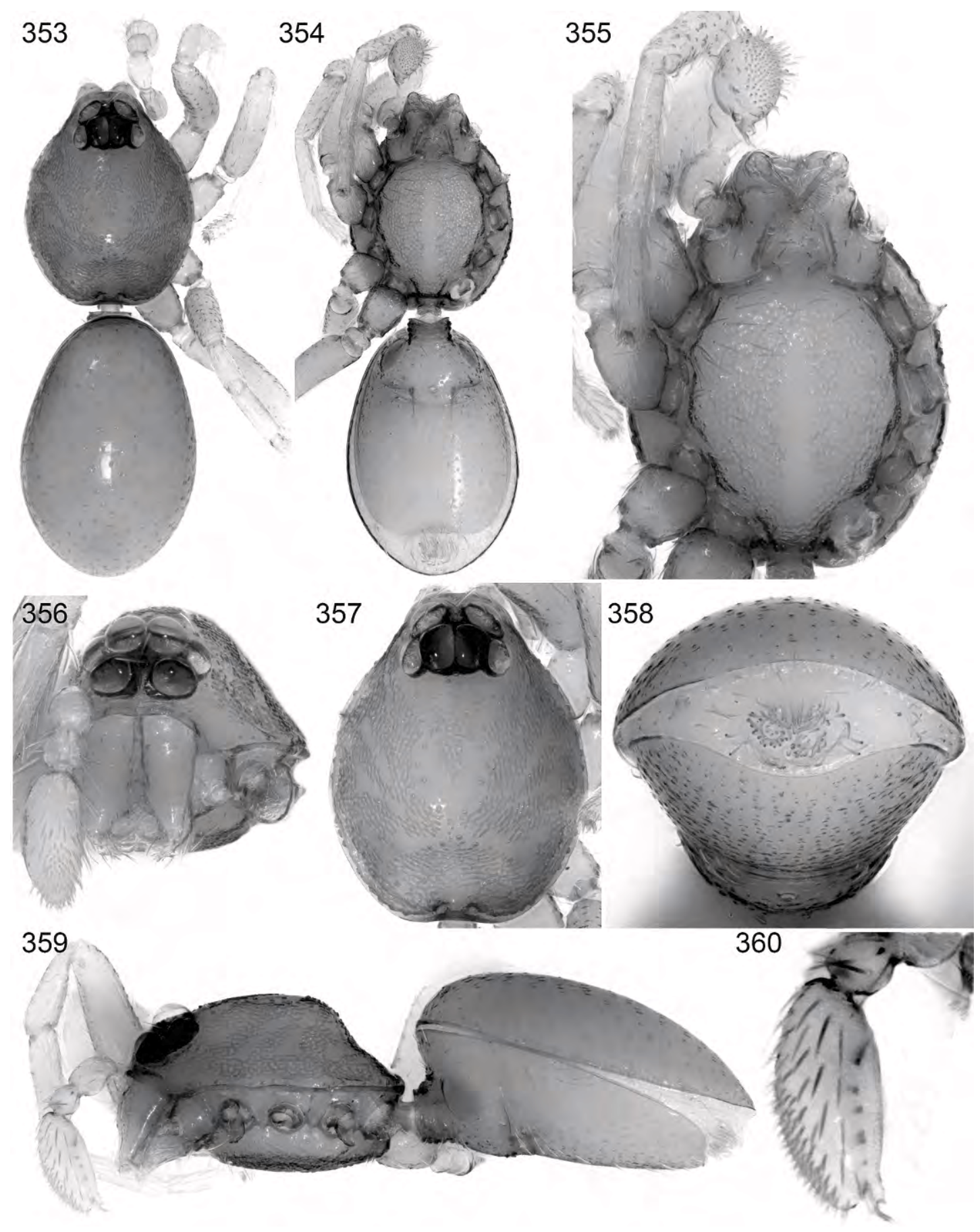

Figs. 353-360. Prethopalpus magnocularis, n. sp., male (PBI_OON 16026). 353. Habitus, dorsal view. 354. Same, ventral view. 355. Prosoma, ventral view. 356. Prosoma, anterior view. 357. Same, dorsal view. 358. Opisthosoma, posterior view. 359. Habitus, lateral view. 360. Male palp, prolateral view. 
Figs. 361-370. Prethopalpus pahang, n. sp., male (PBI_OON 12123). 361. Habitus, dorsal view. 362. Same, ventral view. 363. Same, lateral view. 364. Prosoma, anterior view. 365. Same, ventral view. 366. Same, dorsal view. 367. Same, lateral view. 368. Male palp, prolateral view. 369. Same, dorsal view. 370. Same, retrolateral view.

Malaysia, and Sabah and Sarawak in East Malaysia (map 5).

Prethopalpus leuser Baehr, new species Figures 92-94, 141, 142, 334-352; map 3

TYPES: INDONESIA: Aceh: Male holotype from Gunung Leuser National Park, Ketambe $\left[3.17193^{\circ} \mathrm{N}, 98.39077^{\circ} \mathrm{E}\right], 300 \mathrm{~m}$ (23-30 Nov. 1989, I. Löbl, D. Agosti and D. Burckhardt), deposited in MHNG (PBI_OON 00012083). Female allotype col- lected with holotype, deposited in MHNG (PBI_OON 00023397).

ETYMOLOGY: The specific name is a noun in apposition taken from the type locality.

DiAGNOSIS: Males and females resemble those of $P$. attenboroughi in having a smooth carapace and reduced eyes. Males can be recognized by the bulging distal bulbal tip (figs. 92-94). Females can be separated from those of all other species by the epigastric area having a procurved posterior sclerotization (fig. 141). 


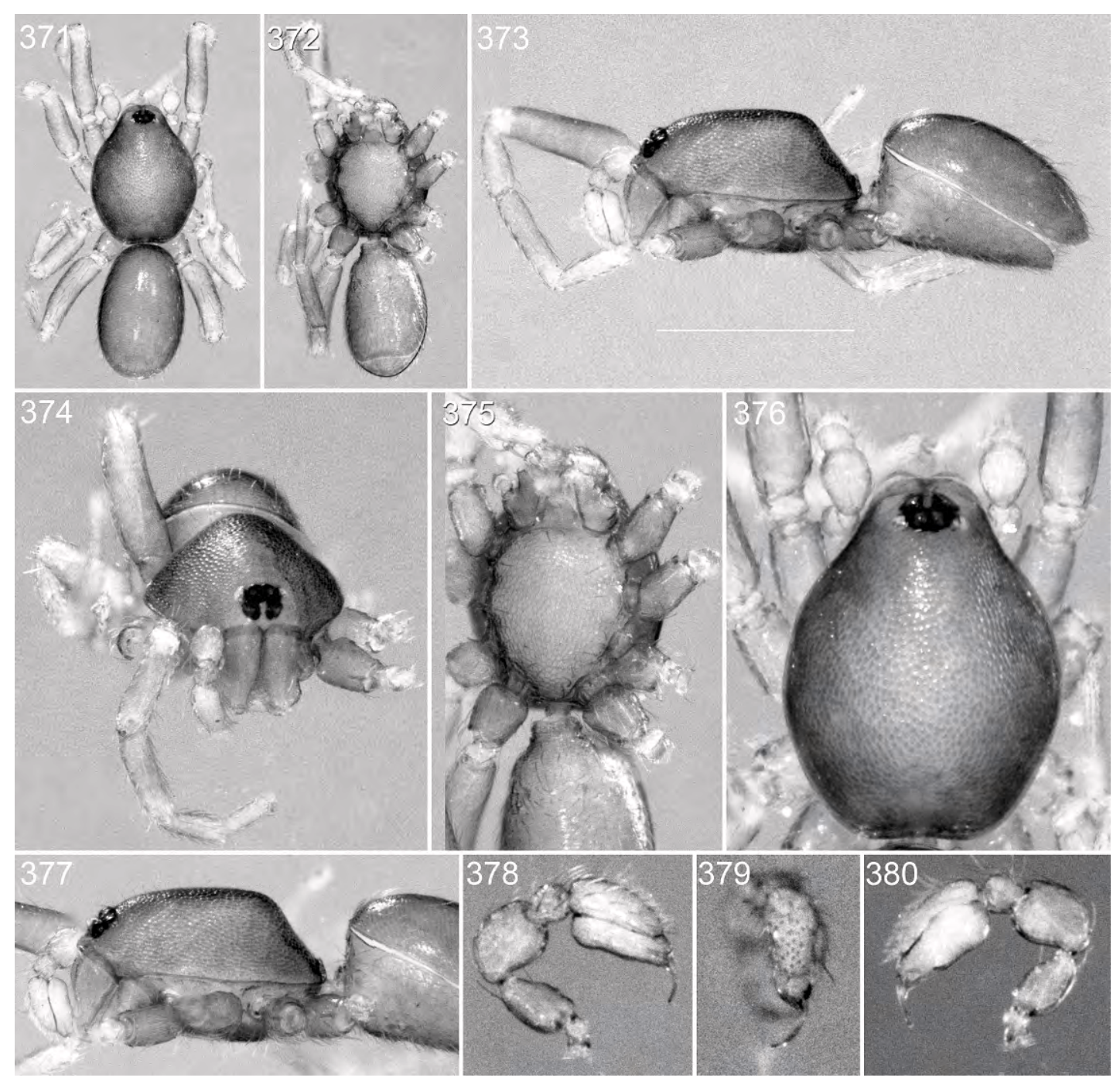

Figs. 371-380. Prethopalpus perak, n. sp., male (PBI_OON 15293). 371. Habitus, dorsal view. 372. Same, ventral view. 373. Same, lateral view. 374. Prosoma, anterior view. 375. Same, ventral view. 376. Same, dorsal view. 377. Same, lateral view. 378. Male palp, prolateral view. 379. Same, dorsal view. 380. Same, retrolateral view.

MAle (PBI_OON 12083, figs. 334-343): Total length 1.8. Prosoma, mouthparts, and abdominal scuta pale orange, surface of elevated portion of pars cephalica smooth, sides finely reticulate; lateral margin without denticles. Eyes: six, small, ALE 0.047; PME 0.022; PLE 0.0164; ALE largest, all eyes circular; posterior eye row straight from both above and front; ALE separated by more than their diameter, ALE-PLE touching,
PME separated by more than their diameter, PLE-PME touching. Sternum longer than wide, surface finely reticulate, without pits, microsculpture absent. Labium rectangular, fused to sternum; endites about twice as long as wide anteromedian tip unmodified. Abdomen ovoid; book lung covers large; pedicel tube short, ribbed. Dorsal scutum covering full length of abdomen, smooth; epigastric scutum not protruding; postepigastric 


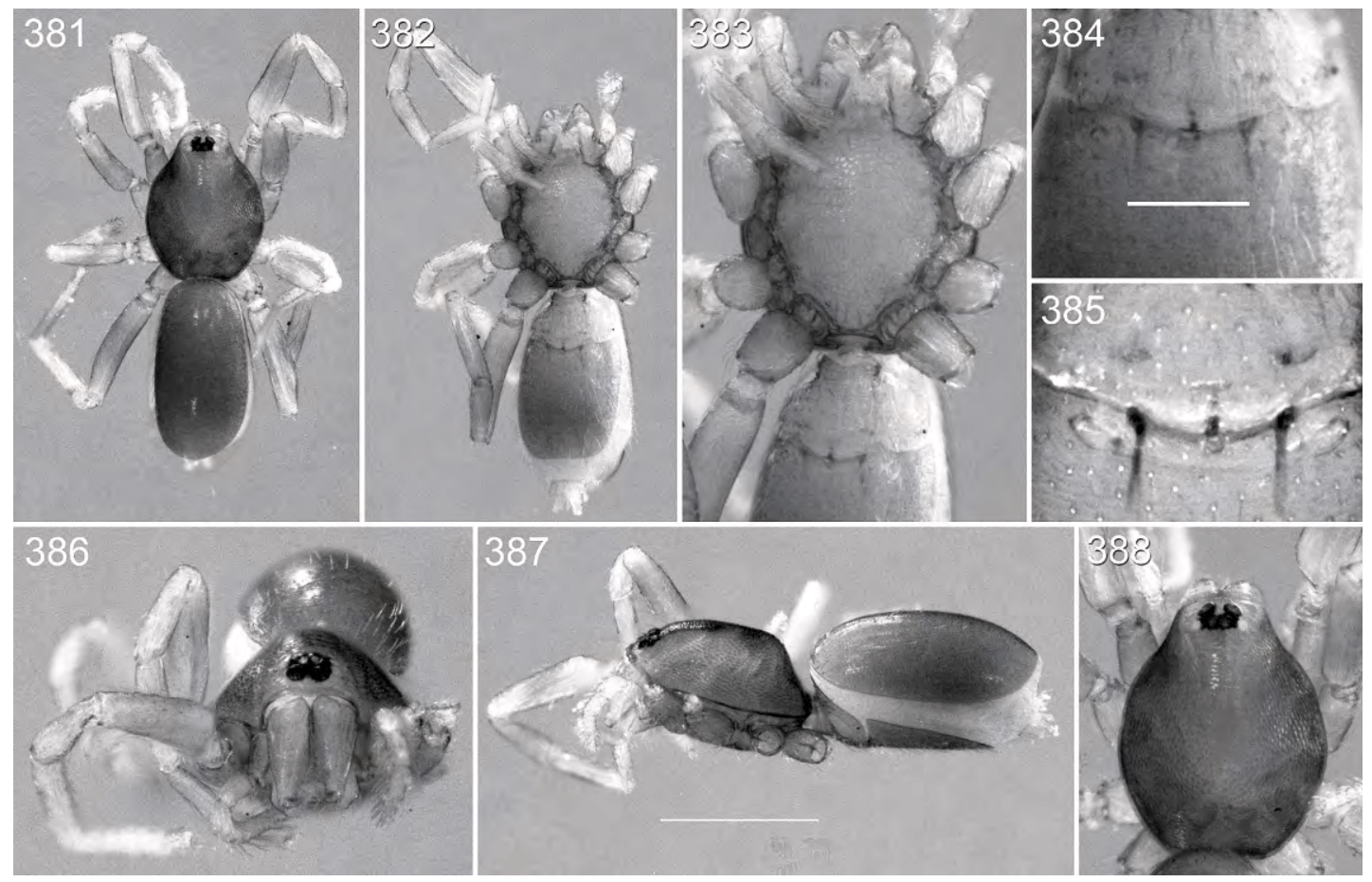

Figs. 381-388. Prethopalpus perak, n. sp., female (PBI_OON 12515). 381. Habitus, dorsal view. 382. Same, ventral view. 383. Prosoma, ventral view. 384. Female epigyne, ventral view. 385. Female epigyne, dorsal view. 386. Prosoma, anterior view. 387. Habitus, lateral view. 388. Prosoma, dorsal view.

scutum long, almost rectangular, covering nearly full length of abdomen, with long posteriorly directed lateral apodemes. Legs pale orange; patella plus tibia I nearly as long as carapace. Palp white; embolus light, femur attaching to patella subbasally; patella longer than femur, enlarged; cymbium not fused with bulb, bulb pyriform with short, broad, medially bent embolus (figs. 92-94).

FEMALE (PBI_OON 23397, figs. 344 352): Total length 0.99. Eyes: ALE 0.029; PME 0.021; PLE 0.016. Epigastric area with procurved posterior sclerotization, internal genital region widely U-shaped, without anterior sclerite (figs. 141, 142).

OTHER MATERIAL EXAMINED: INDONESIA: Aceh: Gunung Leuser National Park, Ketambe [3.17193 $\left.{ }^{\circ} \mathrm{S}, 98.39077^{\circ} \mathrm{E}\right], 300 \mathrm{~m}, 23-30$ Nov. 1989, I. Löbl, D. Agosti and D. Burckhardt, 2 के (MHNG, PBI_OON 00023398).

DisTRIBUTION: Prethopalpus leuser is known only from Gunung Leuser National Park in Sumatra (map 3).

\section{Prethopalpus magnocularis Baehr and Thoma, new species Figures 353-360; map 5}

TYPE: INDONESIA: Kalimantan Timur: Male holotype from Kutal District, Lao Janan, $1^{\circ} 30^{\prime} \mathrm{N}, 116^{\circ} 30^{\prime}$ E (30 Oct. 1979, P. Lehtinen), deposited in UTU (PBI_OON 00016026).

ETYMOLOGY: The specific name is Latin, and refers to the extremely large eyes of this species.

DiAgNosis: This species can be separated from all others by the extremely large eyes (fig. 357).

MAle (PBI_OON 00016026, figs. 353359): Total length 1.49. Prosoma, mouthparts, and abdominal scuta pale orange, broadly oval in dorsal view, pars cephalica strongly elevated in lateral view, posterolateral edge with pair of pits, surface of elevated portion of pars cephalica and sides striate, partly interrupted by small smooth areas; 


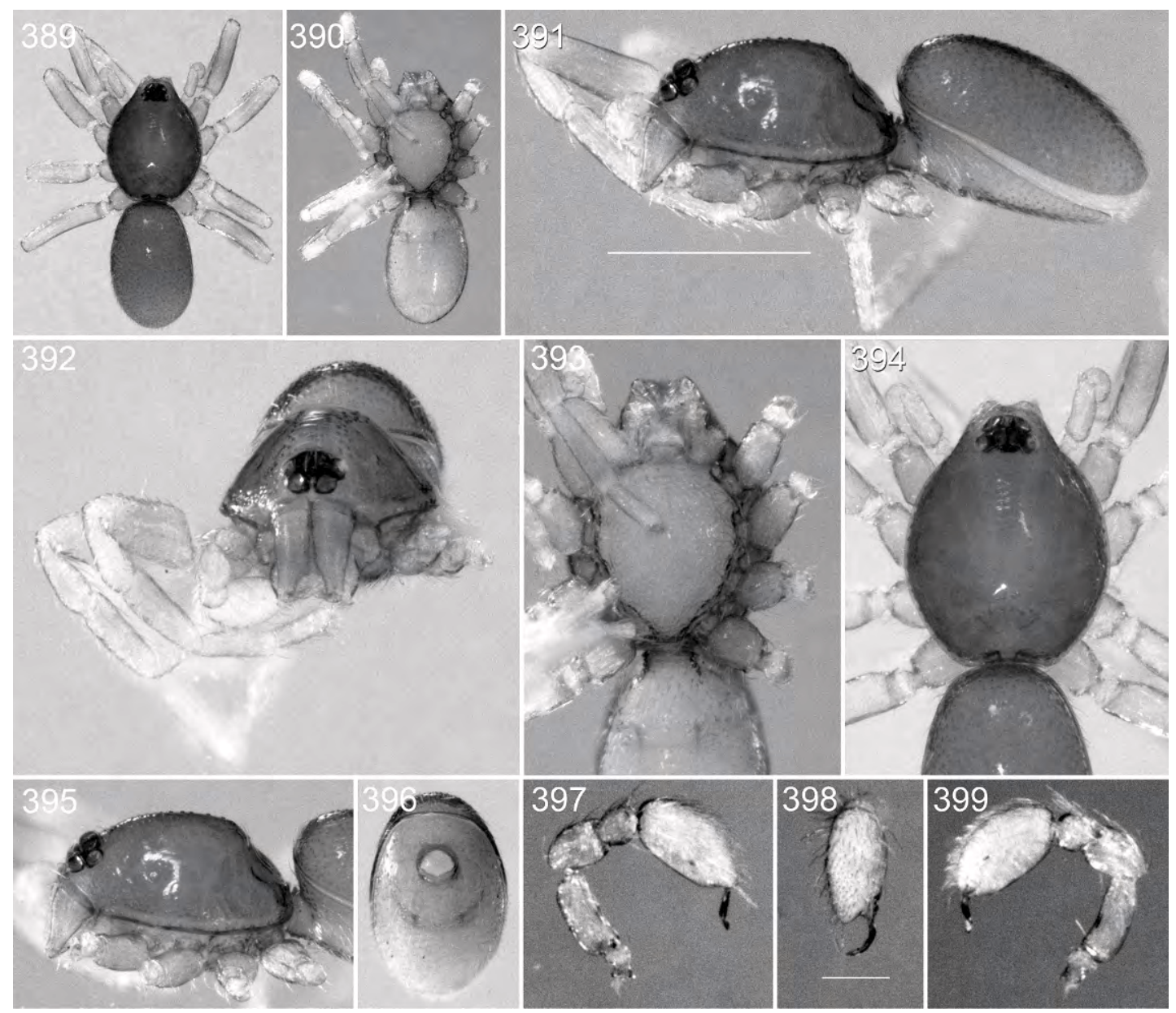

Figs. 389-399. Prethopalpus sabah, n. sp., male (PBI_OON 12344). 389. Habitus, dorsal view. 390. Same, ventral view. 391. Same, lateral view. 392. Prosoma, anterior view. 393. Same, ventral view. 394. Same, dorsal view. 395. Same, lateral view. 396. Opisthosoma, ventral view. 397. Male palp, prolateral view. 398. Same, dorsal view. 399. Same, retrolateral view.

lateral margin with blunt denticles; nonmarginal pars cephalica setae long U-shaped row reaching posterior end of pars cephalica, hair bases equal; nonmarginal pars thoracica setae light, needlelike, very few, scattered; marginal setae originating from anterior base of blunt denticles (= modified hair bases), pointing anteriorly. Clypeus curved downward in front view, vertical in lateral view. Eyes huge, PME largest, PME oval; posterior eye row recurved from above; ALE separated by less than their radius, PLE-PME touching. Sternum without radial furrows between coxae I-II, II-III, III-IV, microsculpture everywhere but middle, lateral margins with three pairs of lateral projections; setae evenly scattered. Chelicerae anterior face with swelling. Abdomen oval; book lung covers round; pedicel short, ribbed. Dorsal scutum covering whole abdomen; postepigastric scutum long, semicircular. Legs pale orange, without color pattern. Epigastric region with oval sperm pore situated at level of anterior spiracles. Palp pale orange; embolus light; femur attaching to patella subbasally; patella shorter than femur, not enlarged; cymbium partly fused to bulb separated by about six pits (fig. 360); row of lateral directed, more strongly sclerotized "ducts," running from one side of the bulb to the other. 


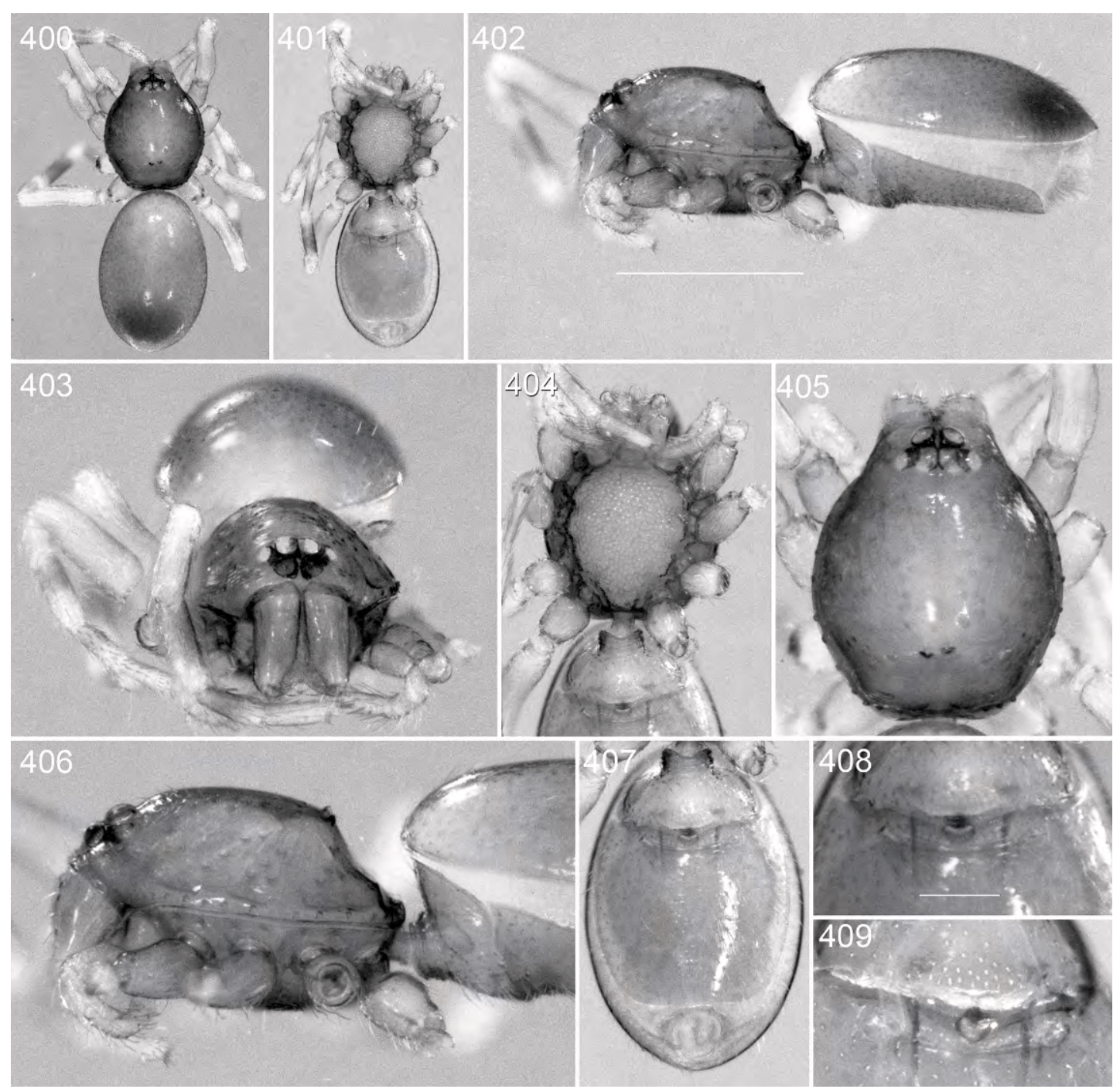

Figs. 400-409. Prethopalpus sabah, n. sp., female (PBI_OON 12313). 400. Habitus, dorsal view. 401. Same, ventral view. 402. Same, lateral view. 403. Prosoma, anterior view. 404. Same, ventral view. 405. Same, dorsal view. 406. Same, lateral view. 407. Opisthosoma, ventral view. 408. Female epigyne, ventral view. 409. Female epigyne, dorsal view.

FEMAle: Unknown.

Other MATERIAl ExAMINED: None.

DISTRIBUTION: Prethopalpus magnocularis is known only from eastern Indonesia Borneo (map 5).

Prethopalpus pahang Baehr, new species Figures 86-88, 361-370; map 3

TYPE: MALAYSIA: Pahang: Male holotype from Frasers Hill, south side, $3.7000^{\circ} \mathrm{N}$,
101.7500 ${ }^{\circ}$ E, 1300 m (26 Aug. 2004, A. Schulz), deposited in MHNG (PBI_OON 00012123).

ETYMOLOGY: The specific name is a noun in apposition taken from the type locality.

Diagnosis: Males resemble those of $P$. bali in having a bifid embolar tip but can be easily recognized by the ventrally bulging bulb (figs. 86,88 ), with a long, thin, medially bent embolus (figs. 86-88).

Male (PBI_OON 12123, figs. 361-370): Total length 1.22. Prosoma, mouthparts, and 


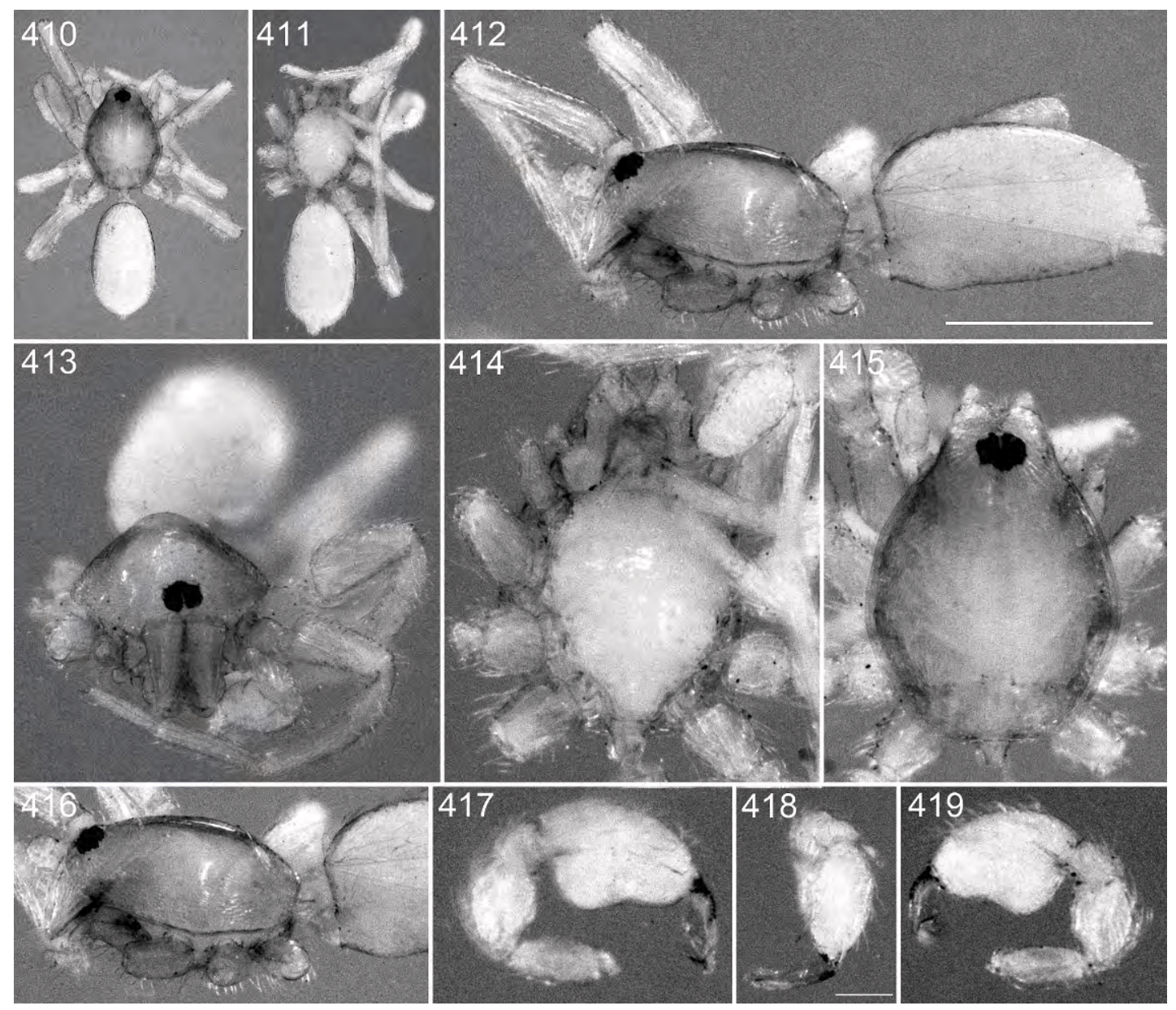

Figs. 410-419. Prethopalpus sarawak, n. sp., male (PBI_OON 22853). 410. Habitus, dorsal view. 411. Same, ventral view. 412. Same, lateral view. 413. Prosoma, anterior view. 414. Same, ventral view. 415. Same, dorsal view. 416. Same, lateral view. 417. Male palp, prolateral view. 418. Same, dorsal view. 419. Same, retrolateral view.

abdominal scuta yellow-brown, surface of elevated portion of pars cephalica smooth, sides granulate; lateral margin without denticles. Eyes: ALE 0.050; PME 0.037; PLE 0.026; ALE largest, ALE oval, PME oval, PLE circular; posterior eye row recurved from above, straight from front; ALE separated by their radius to diameter, ALEPLE separated by less than ALE radius, PME touching for less than half their length, PLE-PME separated by less than PME radius. Sternum longer than wide, surface finely reticulate, without pits. Endites about twice as long as wide, with tiny toothlike projection. Abdomen ovoid; book lung covers large; pedicel tube short, ribbed. Dorsal scutum covering full length of abdomen, smooth; epigastric scutum not protruding; postepigastric scutum long, almost rectangular, covering nearly full length of abdomen. Legs yellow; patella plus tibia I nearly as long as carapace. Palp yellow; embolus light; femur attaching to patella subbasally; patella larger than femur; cymbium not fused with bulb, bulb ventrally bulging, with long thin, medially bent embolus (figs. 86-88).

FEMALE: Unknown.

Other MAterial Examined: SINGAPORE: Bukit Timah Nature Reserve, 


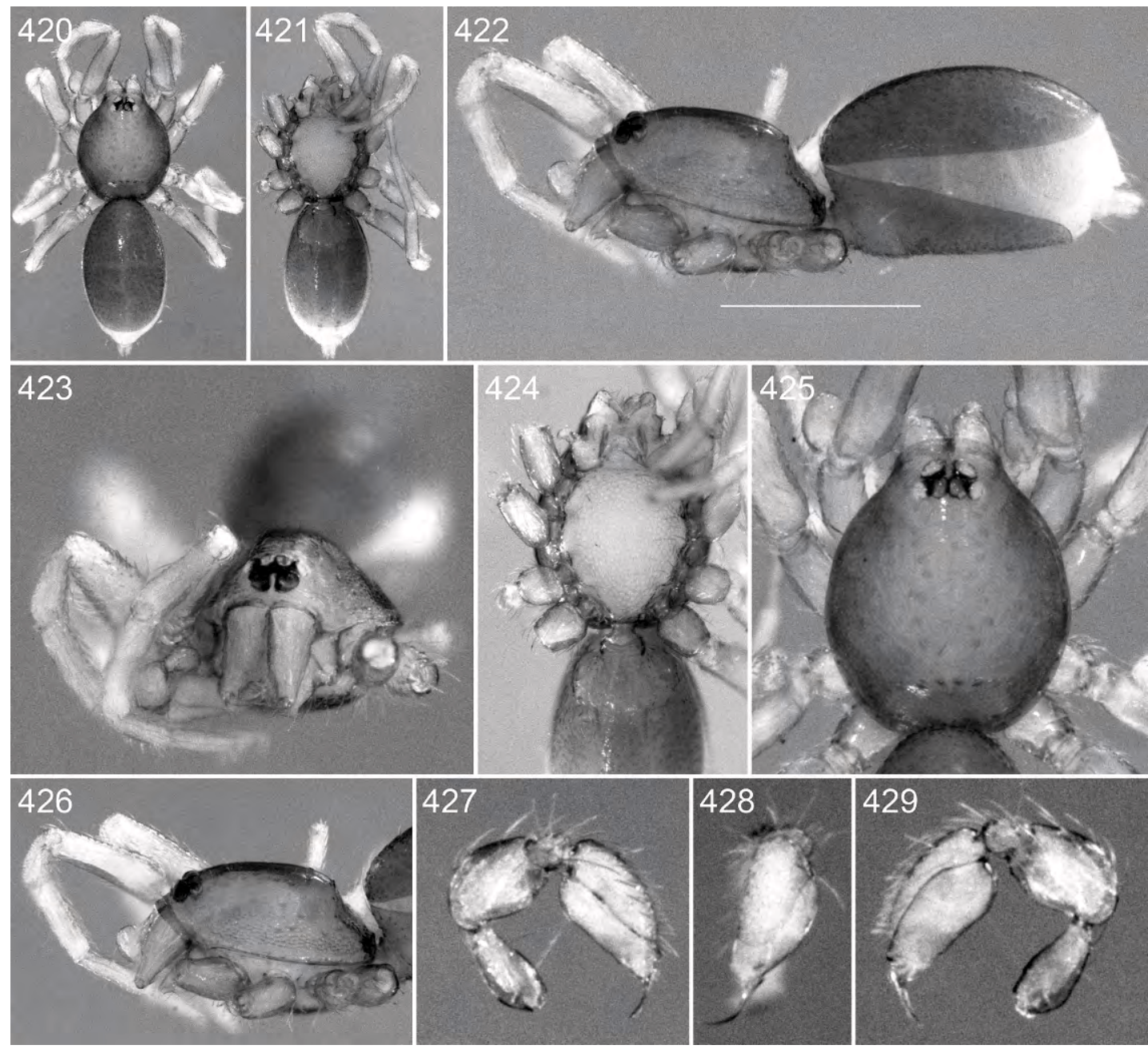

Figs. 420-429. Prethopalpus schwendingeri, n. sp., male (PBI_OON 16192). 420. Habitus, dorsal view. 421. Same, ventral view. 422. Same, lateral view. 423. Prosoma, anterior view. 424. Same, ventral view. 425. Same, dorsal view. 426. Same, lateral view. 427. Male palp, prolateral view. 428. Same, dorsal view. 429. Same, retrolateral view.

$1.38333^{\circ} \mathrm{N}, 103.78333^{\circ} \mathrm{E}, 160 \mathrm{~m}, 162004, \mathrm{~A}$. Schulz, 1 के (MHNG, PBI_OON 00012174).

Distribution: Prethopalpus pahang is known from Peninsular Malaysia and Singapore (map 3).

Prethopalpus perak Baehr, new species Figures 80-82, 151, 152, 371-388; map 3

TYPE: MALAYSIA: Perak: Male holotype from rainforest $\mathrm{NE}$ of Chenderiang, $4.23333^{\circ} \mathrm{N}$, 101.25 E, 290 m (22-31 Jan. 1994, P. Schwendinger), deposited in MHNG (PBI_OON 00015293).
ETYMOLOGY: The specific name is a noun in apposition taken from the federal state in which the type was found.

DiAgnosis: Males and females resembles $P$. kranzae in the strongly granulate carapace sides and top (fig. 376), but can be differentiated by the lack of any spikes or denticles on the carapace (fig. 373).

Male (PBI_OON 15293, figs. 371-380): Total length 1.12. Prosoma, mouthparts, and abdominal scuta yellow-brown, elongate oval in dorsal view, surface of elevated portion of 


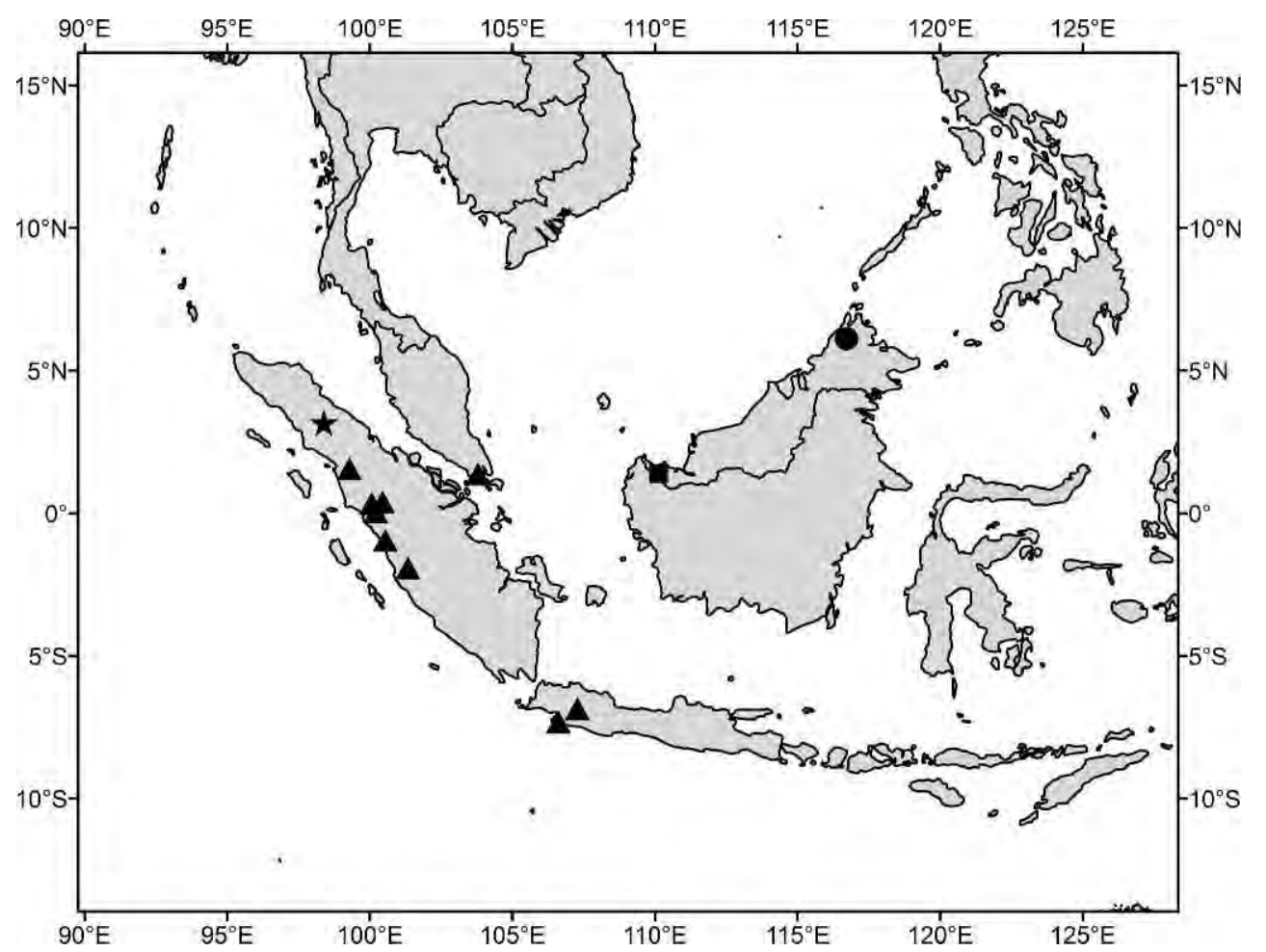

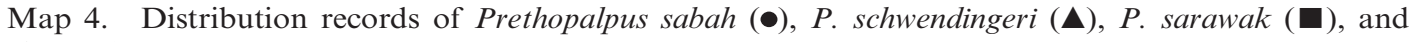
P. $\operatorname{leuser}(\star)$.

pars cephalica and sides granulate; lateral margin without denticles. Eyes: ALE 0.43; PME 0.037; PLE 0.030; ALE largest, all eyes circular; posterior eye row straight from both above and front; ALE separated by their radius to diameter, ALE-PLE separated by less than ALE radius, PME just touching, PLE-PME separated by less than PME radius. Sternum longer than wide, yellowbrown, surface granulate. Endites 1.5 times as long as wide, with tiny toothlike projection. Abdomen ovoid; book lung covers large; pedicel tube short, ribbed. Dorsal scutum, covering full length of abdomen, striated; epigastric scutum not protruding; postepigastric scutum long, almost rectangular, covering nearly full length of abdomen. Legs yellow-brown; patella plus tibia I shorter than carapace. Palp pale orange; embolus light; femur attaching to patella subbasally; patella about as long as femur, enlarged; cymbium, not fused with bulb, bulbal tip with deep retrolateral impression, and thin, medially bent embolus (figs. 80-82).
Female (PBI_OON 12515, figs. 381388): Total length 1.32. Eyes: ALE 0.44; PME 0.033; PLE 0.030. Epigastric area with small, slitlike epigynal opening; short, rounded, internal genital region and $\mathrm{T}$-shaped anterior sclerite (figs. 151, 152).

Other MATERial ExAmined: MALAYSIA: Perak: Cascade de Sungei Simei, Cameron Highlands, $4.78333^{\circ} \mathrm{N}, 101.61666^{\circ} \mathrm{E}, 25$ Mar. 1977, T. Jaccoud, P. Marcuard (MHNG, PBI_OON 00012515, PBI_OON 00015828), 2 \% ; rainforest $\mathrm{NE}$ of Chenderiang, $4.23333^{\circ} \mathrm{N}, 101.25000^{\circ} \mathrm{E}, 330 \mathrm{~m}, 15-22$ Jan. 1995, P. Schwendinger, 1 i (MHNG, PBI_OON 00015565).

DisTRIBUTION: Prethopalpus perak is known only from Perak State in Peninsular Malaysia (map 3).

Prethopalpus sabah Baehr, new species Figures 107-109, 149-150, 389-409; map 4

TyPes: MALAYSIA: Sabah: Male holotype from Poring Hot Springs, Mount 


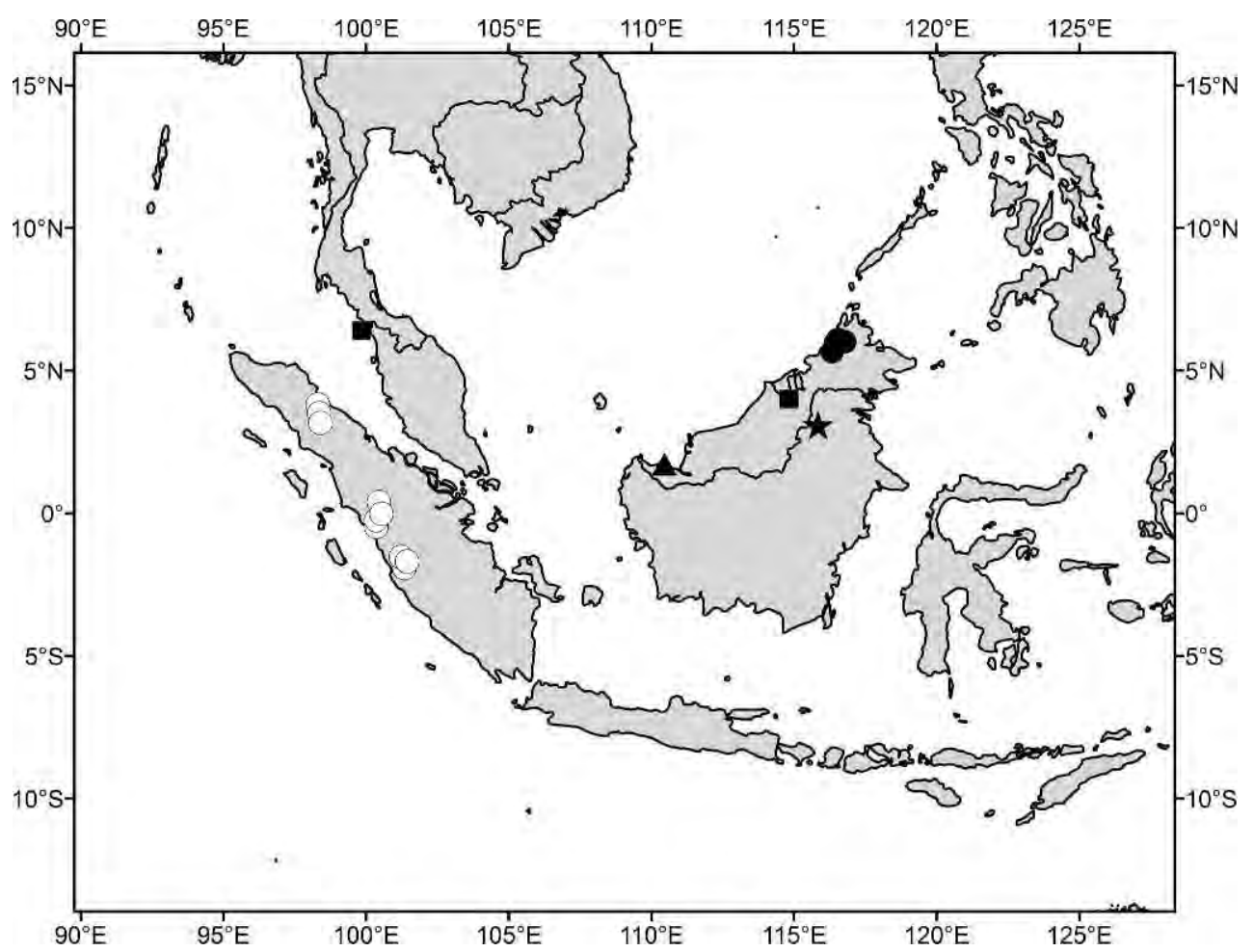

Map 5. Distribution records of Prethopalpus bellicosus $(\bullet)$, P. utara $(\bigcirc)$, P. deelemanae (ム), P. kropfi (ם), and P. magnocularis ( $\star$ ).

Kinabalu National Park $\left[6.11407^{\circ} \mathrm{N}\right.$, $\left.116.72104^{\circ} \mathrm{E}\right], 575 \mathrm{~m}$ (9 May 1987, D. Burckhardt and I. Löbl), deposited in MHNG (PBI_OON 00012344). Female allotype collected with holotype, deposited in MHNG (PBI_OON 00012313).

ETYMOLOGY: The specific name is a noun in apposition taken from the federal state in which the types were collected.

DiAgNOSIS: Males and females resemble those of $P$. brunei in having a smooth pars cephalica and large eyes, but can be easily recognized by the recurved posterior eye row (fig. 394), the much longer palpal femur of males (figs. 107, 109), and the wide, slitlike epigynal opening of females (fig. 149).

Male (PBI_OON 12344, figs. 389-399): Total length 1.9. Prosoma, mouthparts, and abdominal scuta yellow-brown, pars cephalica strongly elevated in lateral view, surface smooth but slightly granulate along the lateral margins; one shallow impression anterolaterally and one slitlike impression posteriorly; lateral margin with blunt denticles. Eyes: ALE 0.052; PME 0.034; PLE 0.045; ALE largest, ALE circular, PME squared, PLE circular; posterior eye row recurved from above, straight from front; ALE separated by their radius to diameter, ALE-PLE separated by less than ALE radius, PME touching throughout most of their length, PLE-PME separated by less than PME radius. Sternum longer than wide, surface finely reticulate, without pits, microsculpture everywhere but front. Endites twice as long as wide, with tiny toothlike projection. Abdomen ovoid; book lung covers large; pedicel tube short, ribbed. Dorsal scutum, covering full length of abdomen smooth; epigastric scutum not protruding; postepigastric scutum long, almost rectangular, covering nearly full length of abdomen. Legs yellow; patella plus tibia I shorter than carapace. Palp yellow; embolus dark; femur attaching to patella subbasally, femur about 1.5 times as long as patella connected 


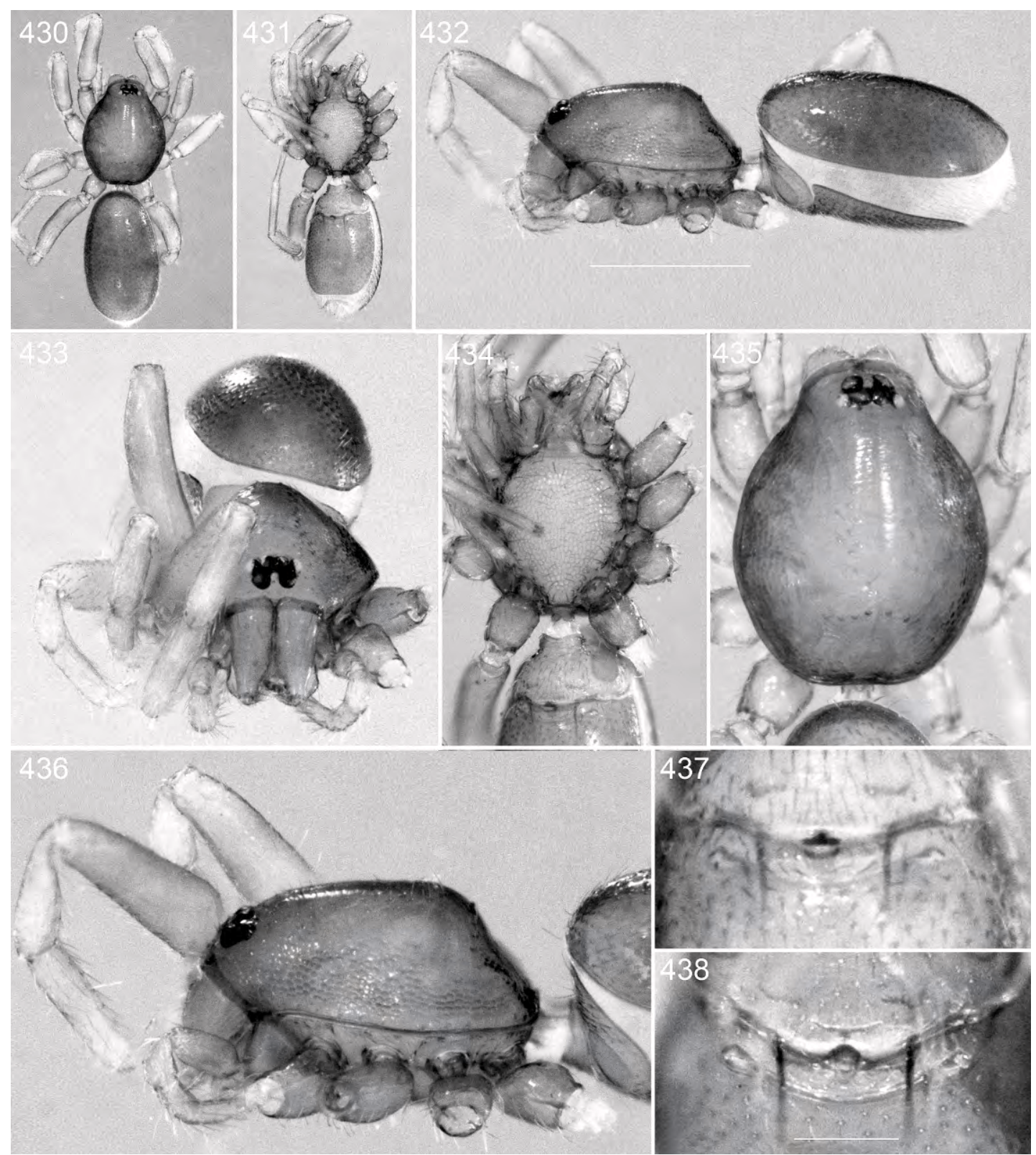

Figs. 430-438. Prethopalpus schwendingeri, n. sp., female (PBI_OON 12426). 430. Habitus, dorsal view. 431. Same, ventral view. 432. Same, lateral view. 433. Prosoma, anterior view. 434. Same, ventral view. 435. Same, dorsal view. 436. Same, lateral view. 437. Female epigyne, ventral view. 438. Female epigyne, dorsal view.

subbasally, patella slightly swollen. Cymbium separated from bulb only by two retrolateral pits and one prolateral pit; embolus thin, bent medially (figs. 107-109).
Female (PBI_OON 12313, figs. 400409): Total length 1.44. Eyes: ALE 0.050; PME 0.052; PLE 0.040. Epigastric area with broad, slitlike epigynal opening with inverted 
Figs. 439-448. Prethopalpus utara, n. sp., male (PBI_OON 12085). 439. Habitus, dorsal view. 440. Same, ventral view. 441. Same, lateral view. 442. Prosoma, anterior view. 443. Same, ventral view. 444. Same, dorsal view. 445. Same, lateral view. 446. Male palp, prolateral view. 447. Same, dorsal view. 448. Same, retrolateral view.

dropshaped, internal genital structure, ending in T-shaped anterior sclerite (figs. 149, 150).

Other Material ExAmined: None.

DisTRIBUTION: Prethopalpus sabah is known only from the type locality in Sabah (map 4).

Prethopalpus sarawak Baehr, new species Figures 95-97, 410-419; map 4

TYPE: MALAYSIA: Sarawak: Male holotype from Gua Bungon District, $1.38722^{\circ} \mathrm{N}$, $110.11944^{\circ}$ E (20 Apr. 1950, M.W.F. Tweedie), deposited in QM (S79886, PBI_OON 00022853).
ETYMOLOGY: The specific name is a noun in apposition taken from the federal state in which the types were collected.

DiAGNOSIS: Males can be easily recognized by the relatively short patella, which is only about as long as the femur (figs. 95, 97), and the very long, medially bent embolus (which is as long as the bulb), and the presence of a velum (figs. 95-97).

MALE (PBI_OON 22853, figs. 410-419): Total length 1.20. Prosoma, mouthparts, and abdominal scuta pale orange, ovoid in dorsal view, surface of elevated portion of pars cephalica smooth, sides finely reticulate; lateral margin without denticles. Clypeus 


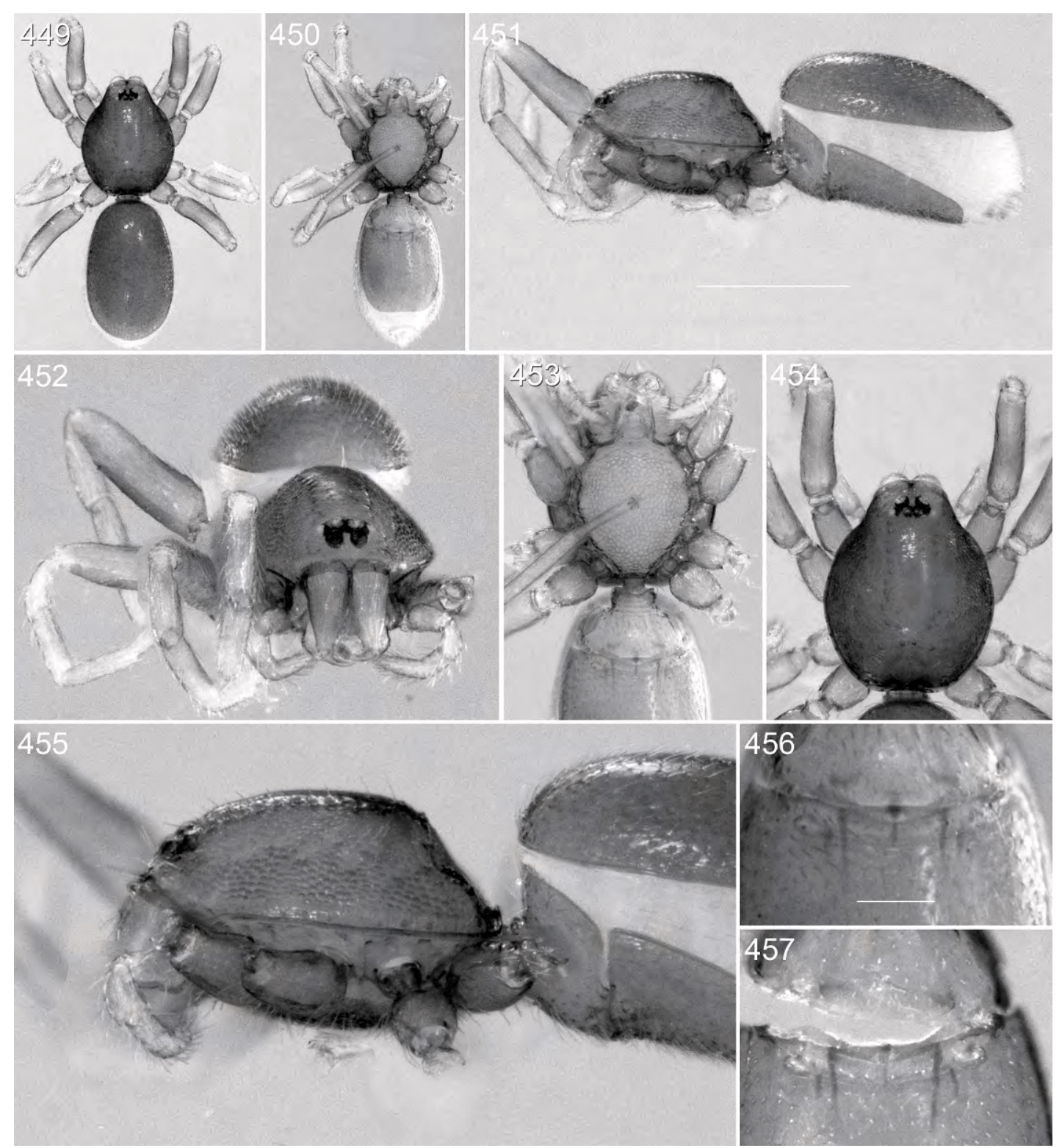

Figs. 449-457. Prethopalpus utara, n. sp., female (PBI_OON 23392). 449. Habitus, dorsal view. 450. Same, ventral view. 451. Same, lateral view. 452. Prosoma, anterior view. 453. Same, ventral view. 454. Same, dorsal view. 455. Same, lateral view. 456. Female epigyne, ventral view. 457. Female epigyne, dorsal view.

margin unmodified, straight in front view, sloping forward in lateral view. Eyes: six, small, ALE 0.034, PME 0.020, PLE 0.020; ALE largest, ALE circular, PME oval, PLE circular; posterior eye row procurved from above, straight from front; ALE separated by their radius to diameter, ALE-PLE separated by less than ALE radius, PME touching throughout most of their length, PLE-PME separated by less than PME radius. Sternum longer than wide, surface not smooth, without pits. Endites twice as long as wide, toothlike projection tiny. Abdomen ovoid, book lung covers large; pedicel tube short, ribbed. Dorsal scutum covering full length of abdomen smooth; epigastric scutum 


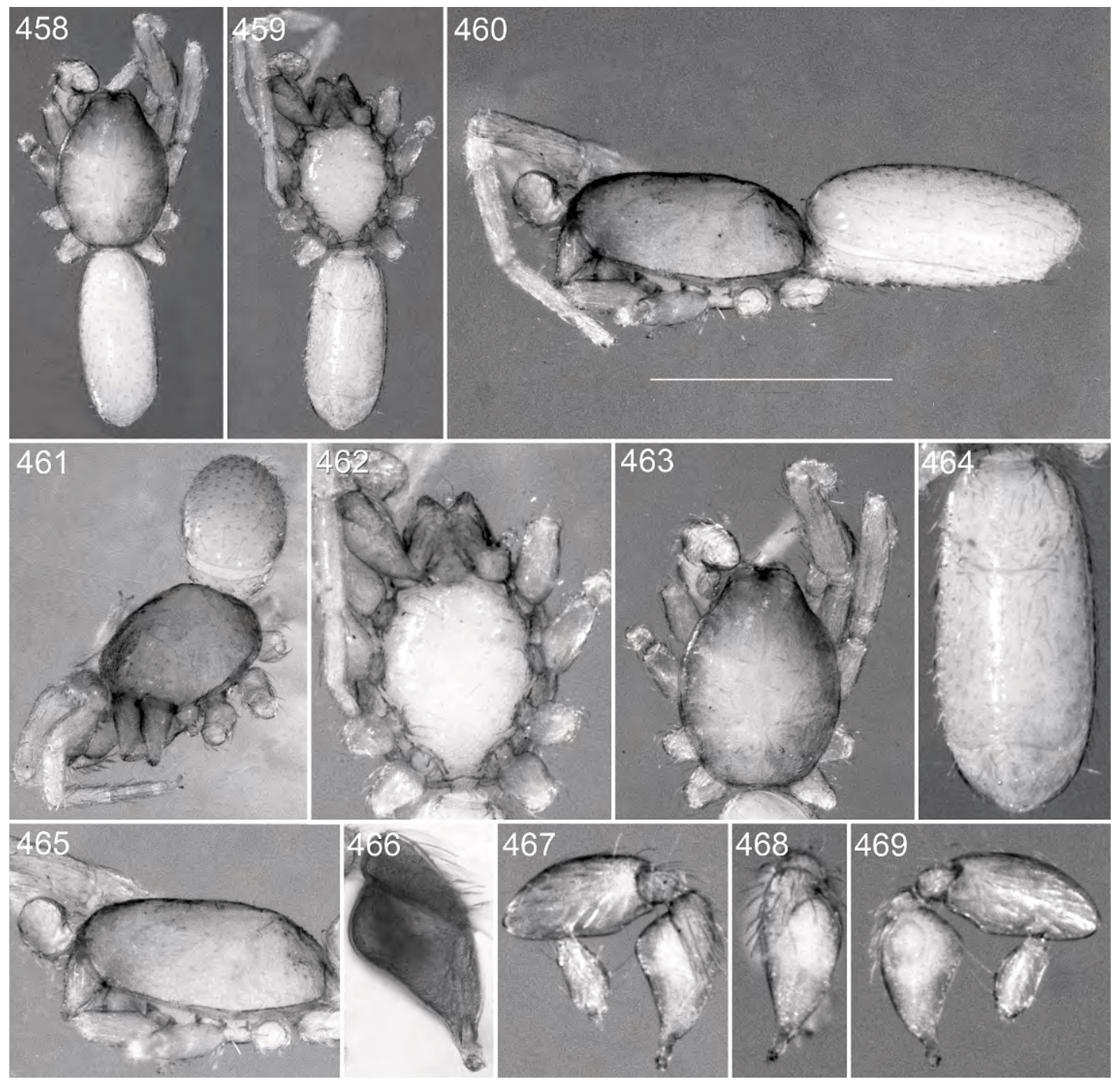

Figs. 458-469. Prethopalpus alexanderi, n. sp., male (PBI_OON 46551). 458. Habitus, dorsal view. 459. Same, ventral view. 460. Same, lateral view. 461. Same, anterior view. 462. Prosoma, ventral view. 463. Prosoma, dorsal view. 464. Abdomen, ventral view. 465. Prosoma, lateral view. 466. Male palpal bulb, prolateral view. 467. Male palp, prolateral view. 468. Male palpal bulb, dorsal view. 469. Male palp, retrolateral view.

not protruding; postepigastric scutum pale orange, long, almost rectangular, covering nearly full length of abdomen, fused to epigastric scutum. Legs white; patella plus tibia I nearly as long as carapace. Palp white; embolus dark; femur attaching to patella subbasally; patella about as long as femur, slightly larger than femur; cymbium not fused with bulb, embolus about as long as bulb with velum, bent medially (figs. 95-97).
FEMALE: Unknown.

Other Material Examined: None.

Distribution: This species is known only from Sarawak (map 4).

Prethopalpus schwendingeri Baehr, new species Figures 77-79, 143, 144, 420-438; map 4

TYPE: INDONESIA: Jawa Barat: Male holotype from Cibodas, Jardin Botanique, 


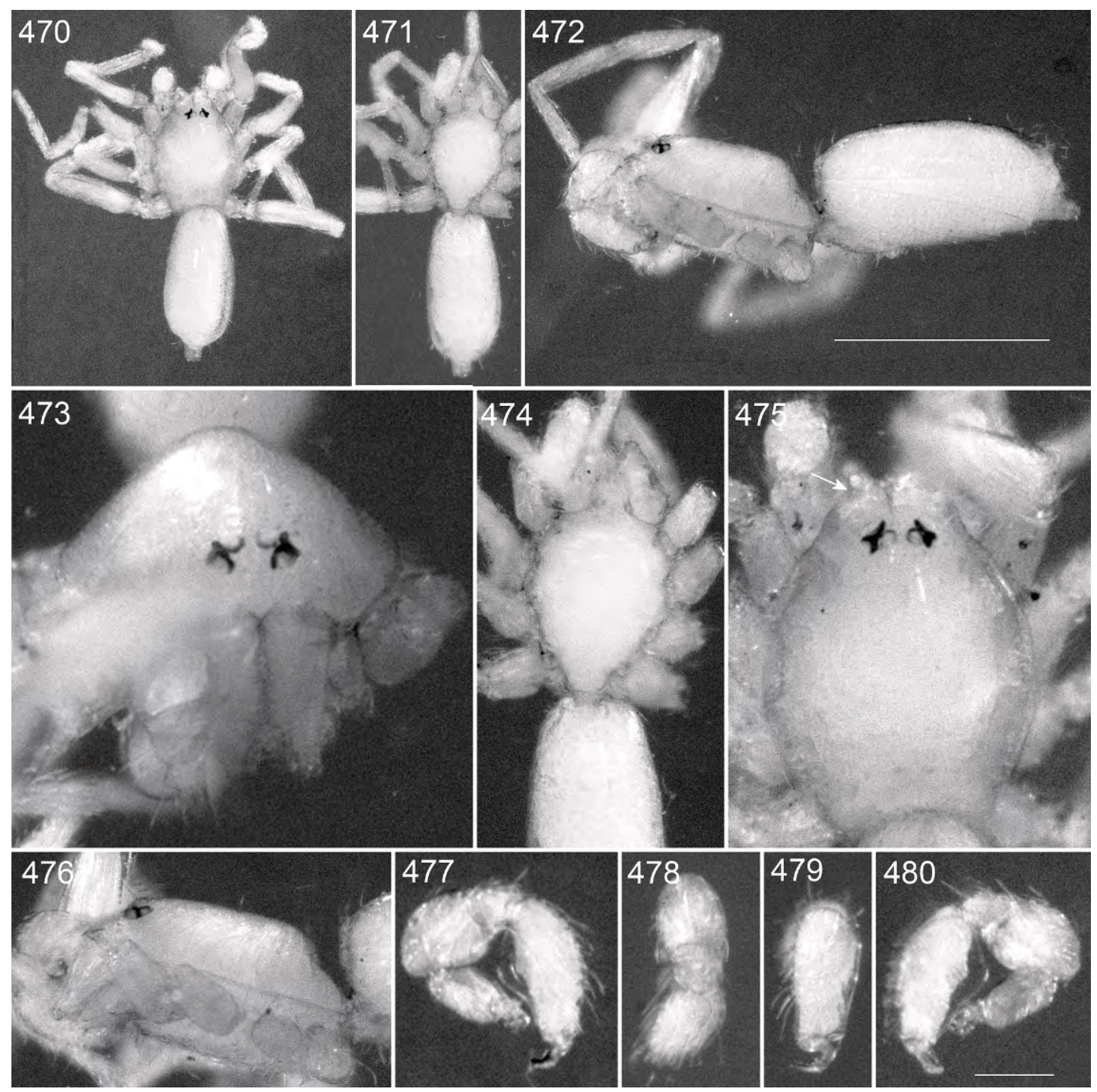

Figs. 470-480. Prethopalpus attenboroughi, n. sp., male (PBI_OON 07438). 470. Habitus, dorsal view. 471. Same, ventral view. 472. Same, lateral view. 473. Prosoma, anterior view. 474. Same, ventral view. 475. Same, dorsal view (arrow $=$ clypeal process). 476. Same, lateral view. 477. Male palp, prolateral view. 478. Patella, dorsal view. 479. Palp, dorsal view. 480. Same, retrolateral view.

7.31666 ${ }^{\circ}$ S, $106.61666^{\circ} \mathrm{E}, 1380 \mathrm{~m}$ (26 Nov. 1987, B. Hauser), deposited in MHNG (Sar87/21, PBI_OON 00016192).

ETYMOLOGY: The specific name is a patronym in honor of Peter J. Schwendinger, senior curator of the arthropod collections at the Muséum d'Histoire Naturelle (Geneva, Switzerland) and an outstanding collector of Asian spiders.

DiAgNOSIS: Males and females resemble those of $P$. fosuma in general body shape and eye sizes, but can be recognized by having the carapace sides being continuously granulate (fig. 425, 426).

MALE (PBI_OON 16192, figs. 420-429): Total length 1.34. Prosoma, mouthparts, and abdominal scuta pale orange, surface of elevated portion of pars cephalica smooth, sides granulate; lateral margin without denticles. Eyes: six, well developed nearly same size, ALE 0.045, PME 0.040, PLE 0.030, 


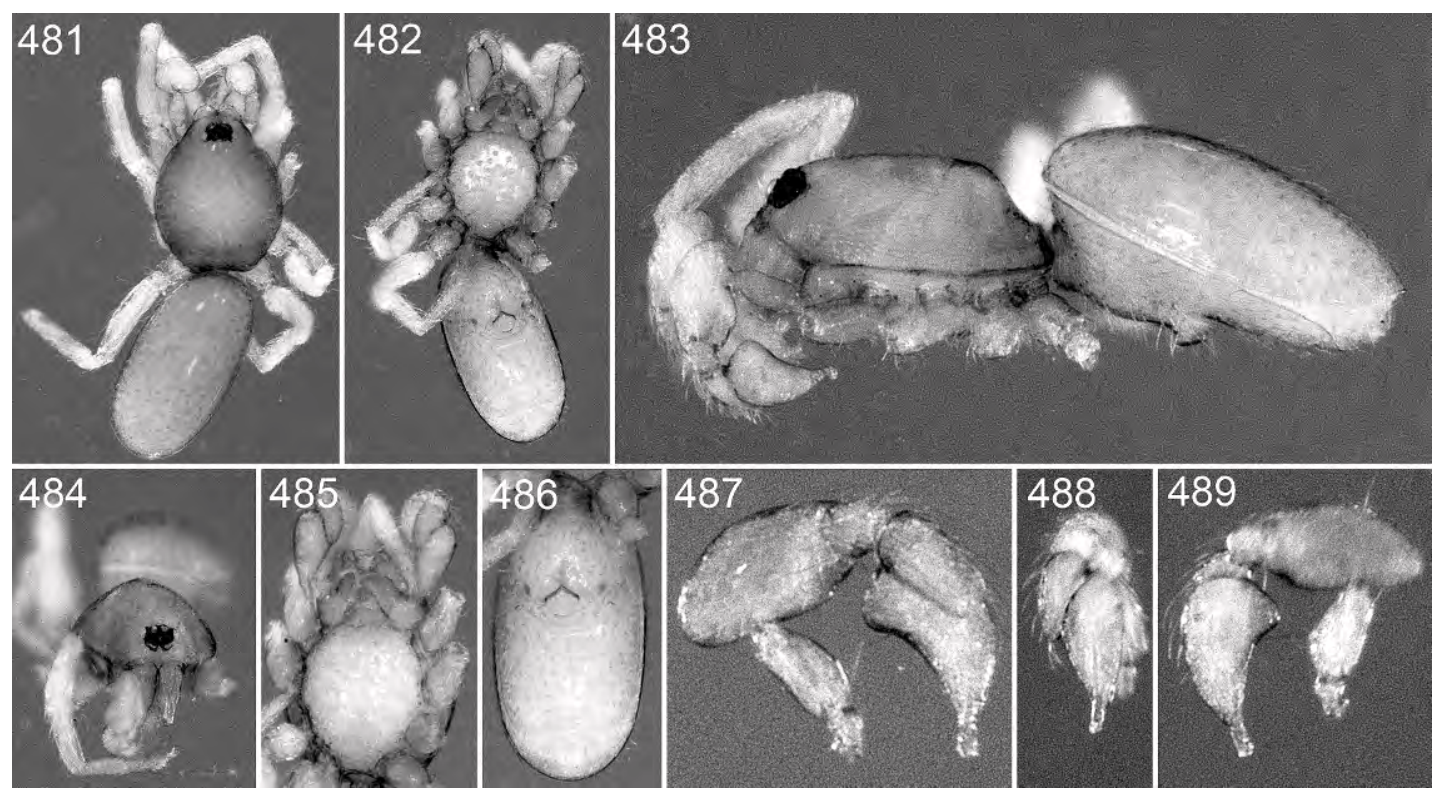

Figs. 481-489. Prethopalpus blosfeldsorum, n. sp., male (PBI_OON 06425). 481. Habitus, dorsal view. 482. Same, ventral view. 483. Same, lateral view. 484. Prosoma, anterior view. 485. Same, ventral view. 486. Opisthosoma, ventral view. 487. Male palp, prolateral view. 488. Same, dorsal view. 489. Same, retrolateral view.

ALE circular, PME oval, PLE circular; posterior eye row straight from both above and front; ALE separated by less than their radius, ALE-PLE separated by less than ALE radius, PME touching throughout most of their length, PLE-PME touching. Sternum longer than wide, surface finely reticulate. Endites twice as long as wide, toothlike projection tiny. Abdomen ovoid; book lung covers large; pedicel tube short, ribbed. Dorsal scutum, covering more than $3 / 4$ of abdomen, smooth but setae originate from small pits; epigastric scutum not protruding; postepigastric scutum long, semicircular, covering about $3 / 4$ of abdomen. Legs yellow; patella plus tibia I shorter than carapace. Palp yellow; embolus light; femur attaching to patella subbasally; patella about as long as femur, enlarged; cymbium not fused with bulb, oval, pear shaped with deep distal groove and medially bent embolus (figs. 74-79).

Female (PBI_OON 12426, figs. 430 438): Total length 1.45. Eyes: ALE 0.044; PME 0.040; PLE 0.029. Epigastric area with broadly oval epigynal opening; internal genital region oval, with $\mathrm{T}$-shaped anterior sclerite with anteriorly directed lateral tips (figs. 143, 144).

Other MATERIAL EXAMINED: INDONESIA: Jawa Barat: Cibodas, $50 \mathrm{~km}$ E Bogor, $6.85^{\circ} \mathrm{S}, 107.28333^{\circ} \mathrm{E}, 1400 \mathrm{~m}, 3-11$ Nov. 1989 , Agosti, Löbl, Burckhardt, 1 के (MHNG, PBI_OON 00012084); Col du Puncak et Cibodas, en bordure de reserve, $7.31666^{\circ} \mathrm{S}$, $106.61666^{\circ} \mathrm{E}, 3$ Aug. 1984, J. Robert, 2 ㅇ (MHNG, PBI_OON 00012460, PBI_OON 00012585); Sumatera Barat: Lubuksikaping, $10 \mathrm{~km} \mathrm{~S}, 0.5000^{\circ} \mathrm{N}, 100.20916^{\circ} \mathrm{E}, 500 \mathrm{~m}, 12$ June 2006, P. Schwendinger, 2 ㅇ, 2 s (MHNG, PBI_OON 00012426); Mt. Kerinci, Jambi Province., $1.91666^{\circ} \mathrm{S}, 101.33333^{\circ} \mathrm{E}$, 3,300 m, 12 Nov. 1989, Agosti, Loebl, Burckhardt, 2 (MHNG, PBI_OON 00023400); Mt Merapi, SE of Bukittinggi, $0.39111^{\circ} \mathrm{N}, 100.44111^{\circ} \mathrm{E}, 2150 \mathrm{~m}, 4-5$ June 2006, P. Schwendinger, 1 क (MHNG, PBI_OON 00012422); Mt Merapi, ca. 15 km $\mathrm{SE}$ of Bukittinggi, $0.39222^{\circ} \mathrm{N}, 100.44833^{\circ} \mathrm{E}$, $1650 \mathrm{~m}, 4$ June 2006, P. Schwendinger, 1 ㅇ, 1 के (MHNG, PBI_OON 00012382); Rimbo Panti Nature Reserve, ca. $30 \mathrm{~km} \mathrm{~N}$ of Lubuksikaping, $\quad 0.34611^{\circ} \mathrm{N}, \quad 100.06916^{\circ} \mathrm{E}$, 


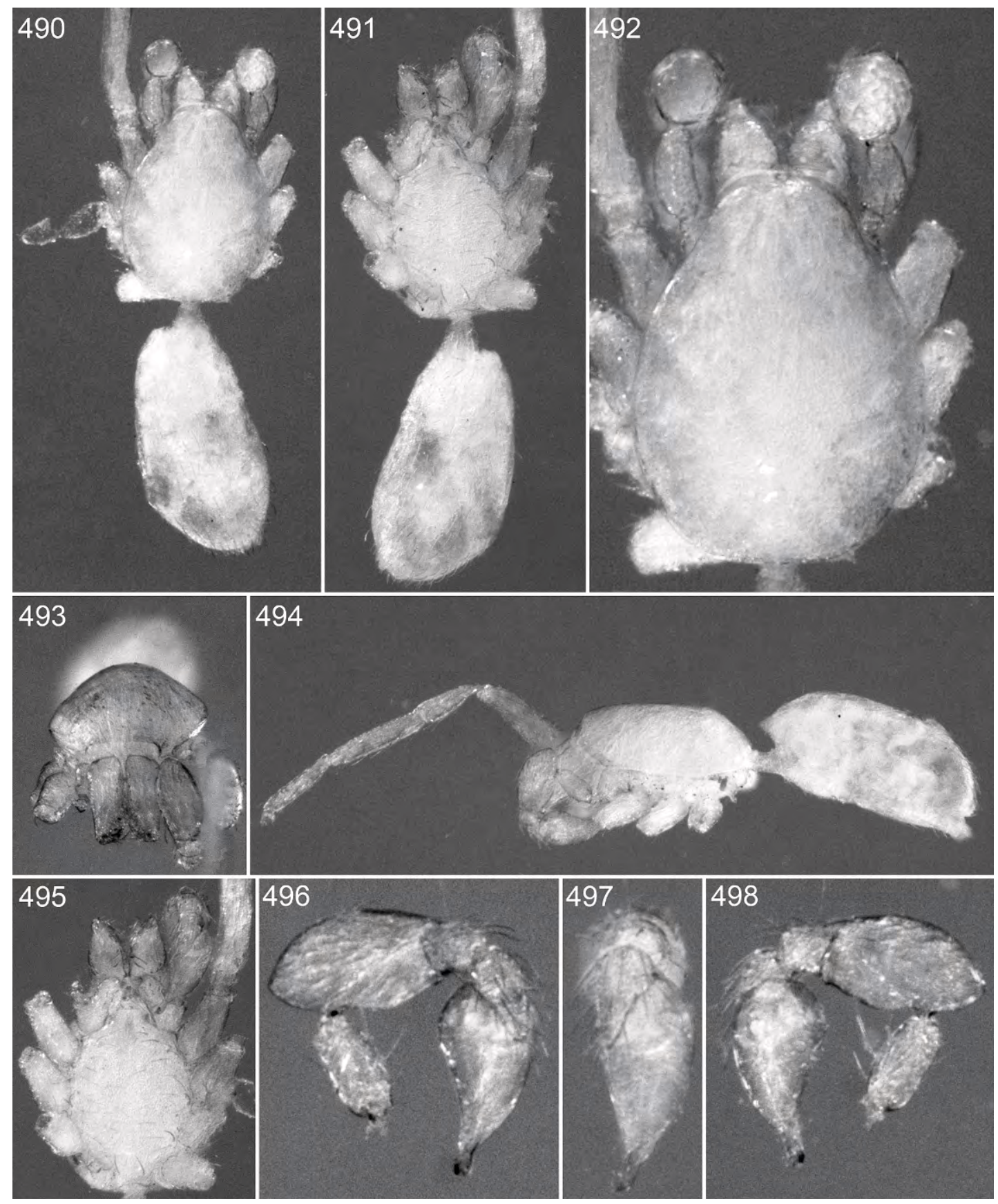

Figs. 490-498. Prethopalpus boltoni, n. sp., male (PBI_OON 46552). 490. Habitus, dorsal view. 491. Same, ventral view. 492. Prosoma, dorsal view. 493. Prosoma, anterior view. 494. Habitus, lateral view. 495. Prosoma, ventral view. 496. Male palp, prolateral view. 497. Same, dorsal view. 498. Male palp, retrolateral view. 


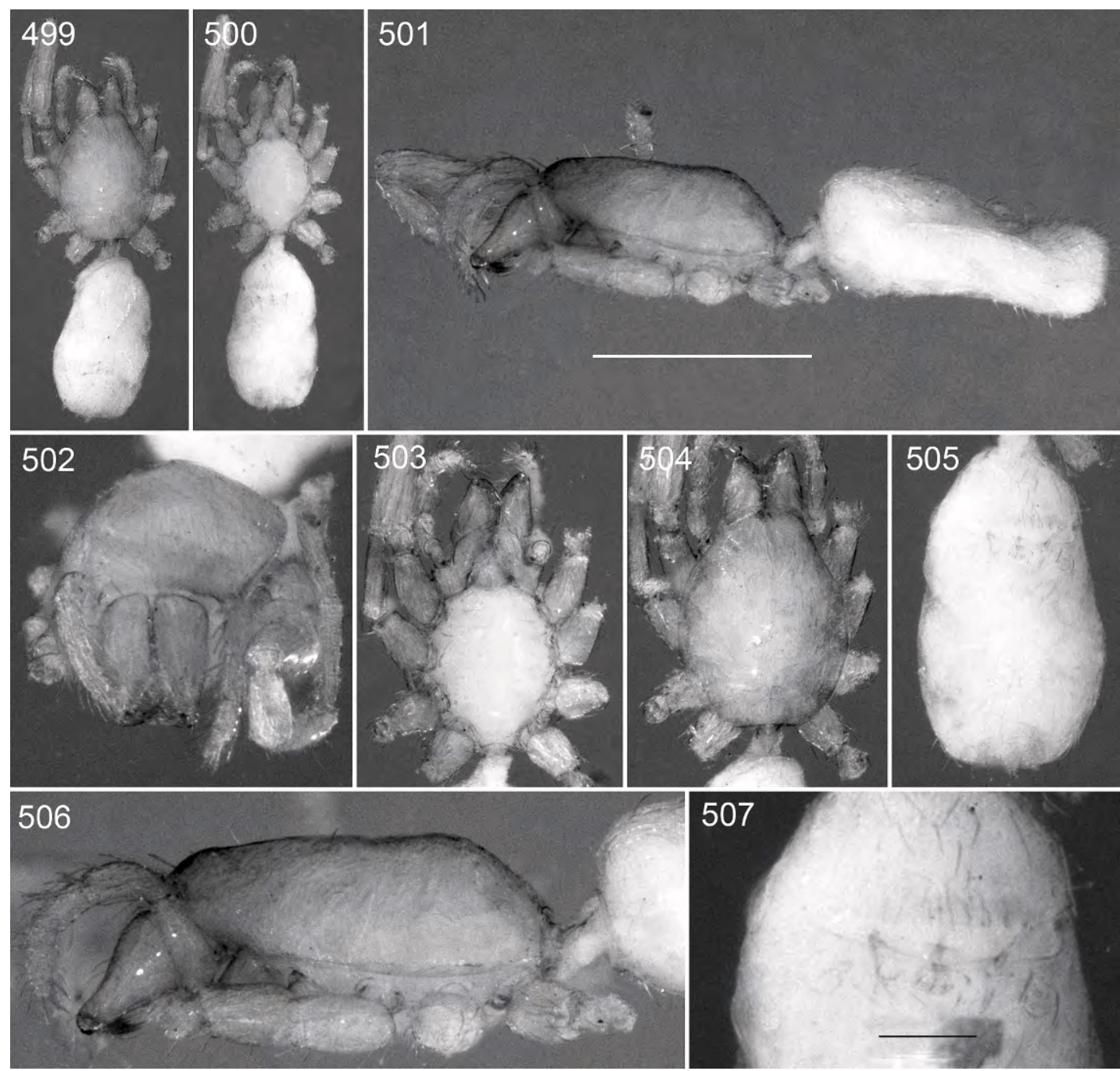

Figs. 499-507. Prethopalpus boltoni, n. sp., female (PBI_OON 18856). 499. Habitus, dorsal view. 500. Same, ventral view. 501. Same, lateral view. 502. Prosoma, anterior view. 503. Same, ventral view. 504. Same, dorsal view. 505. Abdomen, ventral view. 506. Prosoma, lateral view. 507. Female epigyne, ventral view.

$350 \mathrm{~m}, 11-13$ June 2006, P. Schwendinger, 2 s. 1 q (MHNG, PBI_OON 00012307); Taman Hutan Raya Bung Hatta, near road Padang-Lubuksulasih, $0.94583^{\circ} \mathrm{S}, 100.54361^{\circ} \mathrm{E}$, 1,100 m, 29 May 2006, P. Schwendinger, 1 우 (MHNG, PBI_OON 00012416). Sumatera Utara: Sipirok, Dolok Sipirok National Park, ca. $30 \mathrm{~km} \mathrm{~N}$ of Padangsidempuan, $1.56527^{\circ} \mathrm{N}, 99.28416^{\circ} \mathrm{E}, 1,000 \mathrm{~m}, 16$ June 2006, P. Schwendinger, 1 ㅇ (MHNG, PBI_OON 00012335). SINGAPORE: Bukit
Timah, 100 m, 17 Dec. 1987, B. Hauser, 2 s, 1 ㅇ (MHNG, PBI_OON 00016229); Bukit Timah, $1.3666^{\circ} \mathrm{N}, 103.8000^{\circ} \mathrm{E}, 100 \mathrm{~m}, 20 \mathrm{Feb}$. 1988, J. and F. Murphy, 1 oै (AMNH 15529 , PBI_OON 00036605); Bukit Timah Nature Reserve, $1.3333^{\circ} \mathrm{N}, 103.8833^{\circ} \mathrm{E}, 6$ June 1968 , unknown collector, 1 के (MHNG, PBI_OON 00015584); same location, 27 July 1969, unknown collector, 1 ㅇ (MHNG, PBI_OON 00012804); Island Country Club, entre Lower Peirce Reservoir et Windsor Park Estate, 


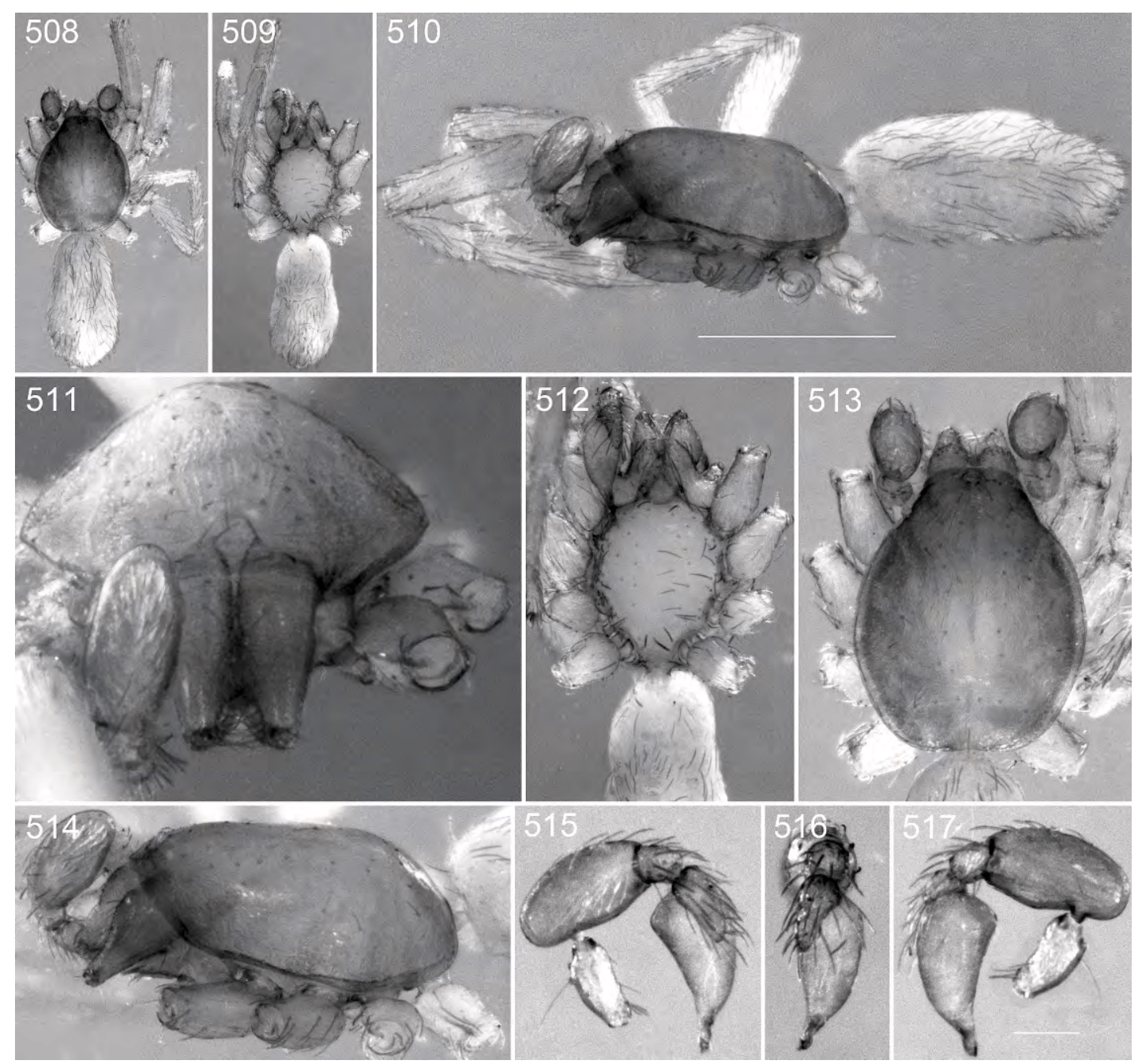

Figs. 508-517. Prethopalpus callani, n. sp., male (PBI_OON 46576). 508. Habitus, dorsal view. 509. Same, ventral view. 510. Same, lateral view. 511. Prosoma, anterior view. 512. Same, ventral view. 513. Same, dorsal view. 514. Same, lateral view. 515. Male palp, prolateral view. 516. Same, dorsal view. 517. Same, retrolateral view.

$1.3000^{\circ} \mathrm{N}, 103.8333^{\circ} \mathrm{E}, 60 \mathrm{~m}, 12$ Nov. $1988, \mathrm{~B}$. Hauser, 1 ㅇ (MHNG, PBI_OON 00015307); Nee Soon Swamp, 27 Dec. 1968, unknown collector, 1 sे (MHNG, PBI_OON 00015570).

DisTRIBUTION: Prethopalpus schwendingeri is known from Java, Sumatra, and Singapore (map 4).

Prethopalpus utara Baehr, new species Figures 89-91, 137-138, 439-457; map 4

TYPES: INDONESIA: Sumatra Utara: Male holotype from Langkat, Reserve natur- elle de Bukit Lawang $\left[3.81666^{\circ} \mathrm{N}, 98.30000^{\circ} \mathrm{E}\right]$, 180 m (20 Nov. 1985, B. Hauser), deposited in MHNG (PBI_OON 00012085). Female allotype collected with holotype, deposited in MHNG (PBI_OON 00023392).

ETYMOLOGY: The specific name is a noun in apposition taken from the type locality.

Diagnosis: Males and females resemble those of $P$. fosuma and $P$. schwendingeri in having a smooth pars cephalica top but can be easily recognized by the more domed carapace (fig. 445), and PLE-PME separated 


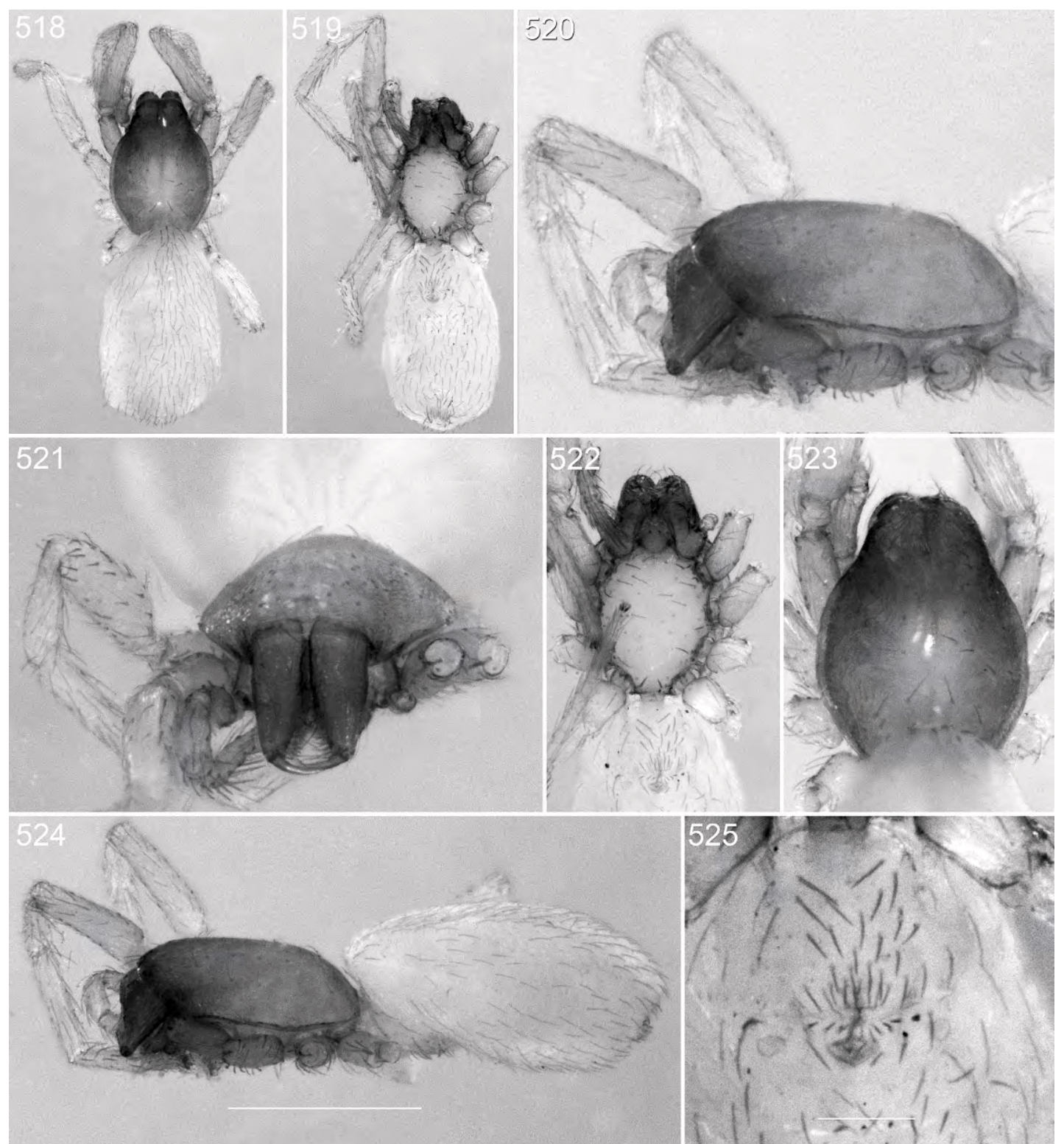

Figs. 518-525. Prethopalpus callani, n. sp., female (PBI_OON 46564). 518. Habitus, dorsal view. 519. Same, ventral view. 520. Prosoma, lateral view. 521. Same, anterior view. 522. Same, ventral view. 523. Same, dorsal view. 524. Habitus, lateral view. 525. Female epigyne, ventral view.

by more than the PME diameter (fig. 444). Males can be separated from both species by the short broad medially bent embolus (figs. 89-91) and females by a long spinelike, internal genital structure reaching the tracheal groove (fig. 138).
Male (PBI_OON 12085, figs. 439-448): Total length 1.15. Prosoma, mouthparts, and abdominal scuta yellow-brown, surface of elevated portion of pars cephalica smooth, sides granulate; lateral margin with blunt denticles. Eyes: ALE 0.043; PME 0.032; PLE 


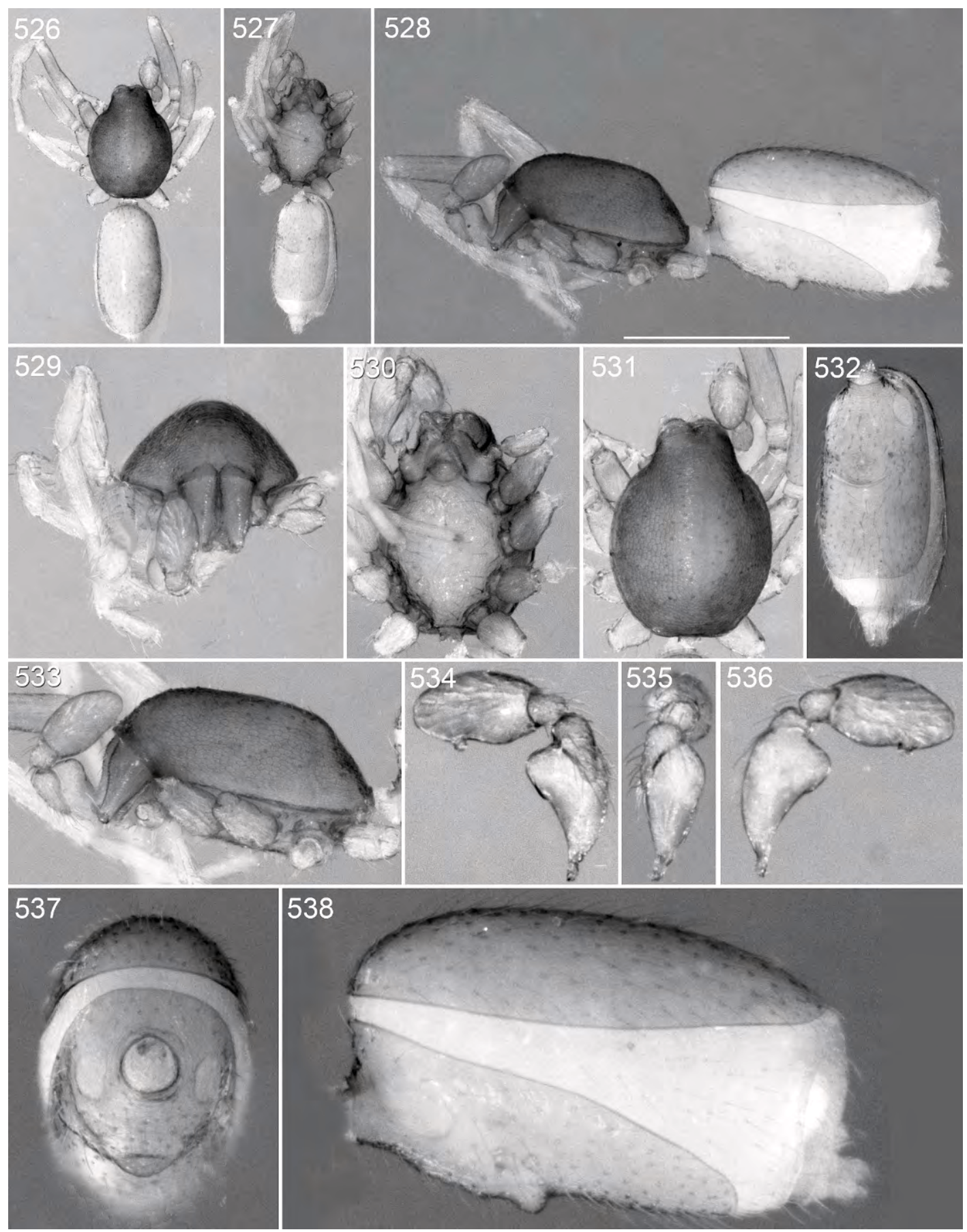

Figs. 526-538. Prethopalpus cooperi, n. sp., male (PBI_OON 46539). 526. Habitus, dorsal view. 527. Same, ventral view. 528. Same, lateral view. 529. Prosoma, anterior view. 530. Prosoma, ventral view. 531. Prosoma, dorsal view. 532. Abdomen, ventral view. 533. Prosoma, lateral view. 534. Male palp, prolateral view. 535. Same, dorsal view. 536. Same, retrolateral view. 537. Abdomen, anterior view. 538. Abdomen, lateral view. 


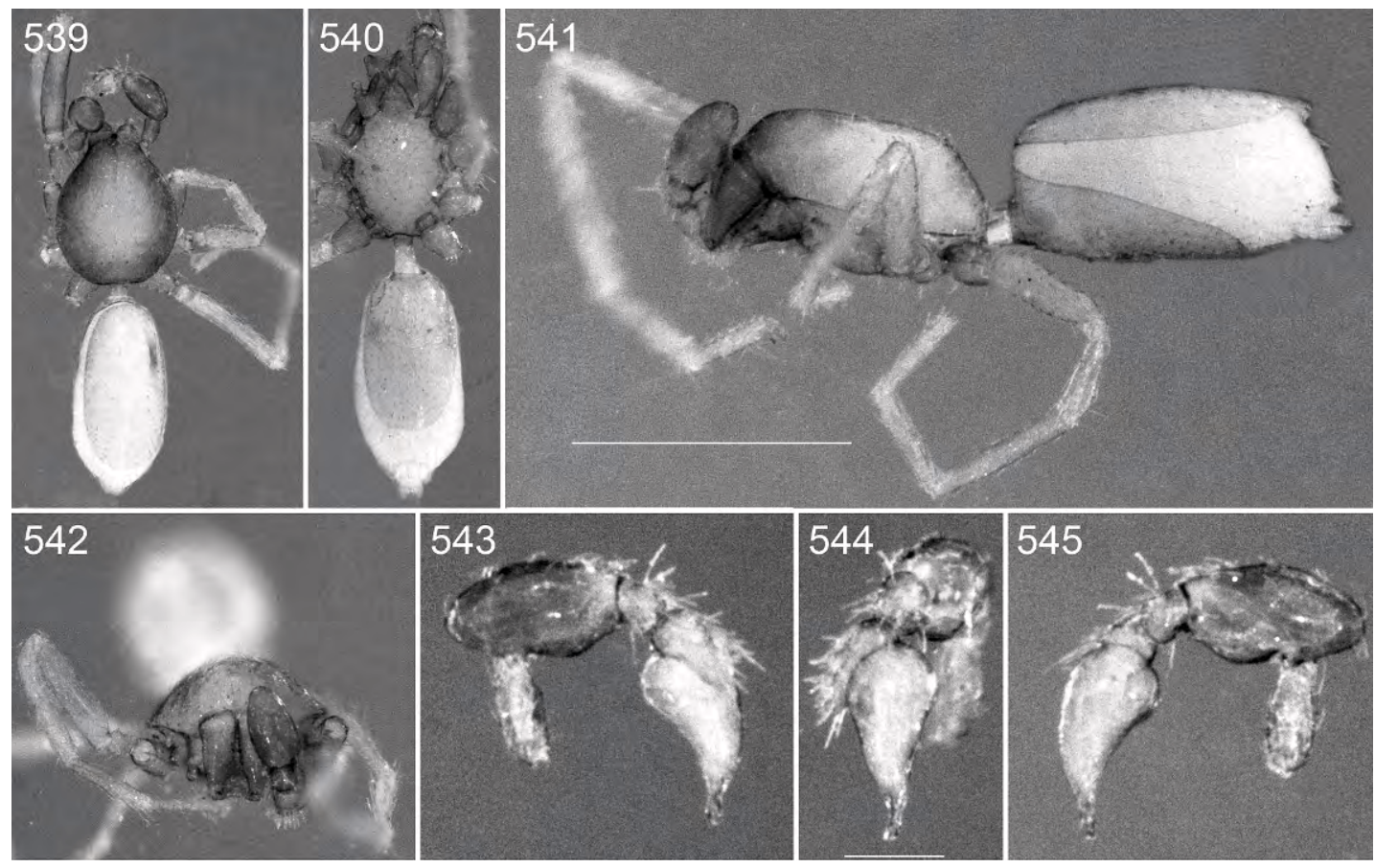

Figs. 539-545. Prethopalpus eberhardi, n. sp., male (PBI_OON 18855). 539. Habitus, dorsal view. 540. Same, ventral view. 541. Same, lateral view. 542. Prosoma, anterior view. 543. Male palp, prolateral view. 544. Same, dorsal view. 545. Same, retrolateral view.

0.031; ALE largest, all eyes circular; posterior eye row straight from above, straight from front; ALE separated by their radius to diameter, ALE-PLE separated by ALE radius to ALE diameter, PME separated by less than their radius, PLE-PME separated by more than PME diameter. Sternum longer than wide, surface strongly reticulate, without pits, microsculpture everywhere but front. Endites twice as long as wide, with very short blunt projection. Abdomen ovoid; book lung covers large; pedicel tube short, ribbed. Dorsal scutum covering full length of abdomen smooth; epigastric scutum not protruding; postepigastric scutum short, almost rectangular, covering nearly full length of abdomen. Legs pale orange; patella plus tibia I nearly as long as carapace. Palp pale orange; embolus light; femur attaching to patella subbasally; patella longer than femur, enlarged; cymbium not fused with bulb, bulb pyriform with deep retrolateral slitlike excavation distally, embolus with broad base and short medially bent tip figs. 89-91).
Female (PBI_OON 23392, figs. 449457): Total length 1.39. Eyes: ALE 0.040; PME 0.036; PLE 0.026. Epigastric area with oval epigynal opening a long spinelike structure reaching tracheal groove and ending in a T-shaped sclerite anteriorly (figs. 137-138).

OTHER MATERIAl EXAMINED: INDONESIA: Sumatera Utara: Reserve naturelle de Bukit Lawang, $3.81666^{\circ} \mathrm{N}, 98.30000^{\circ} \mathrm{E}, 180 \mathrm{~m}, 20$ Nov. 1985, B. Hauser, 1 s (MHNG, PBI_OON 00023393). Sumatera Barat: Anai Valley, $6 \mathrm{~km} \mathrm{~S}$ of Pandangpanjang, $0.47722^{\circ} \mathrm{S}$, $100.35388^{\circ} \mathrm{E}$, 1 June 2006, P. Schwendinger, 1 s, 1 q (MHNG, PBI_OON 00012398); Batang, Palupuh Rafflesia Sanctuary, 12 km $\mathrm{N}$ of Bukittinggi, $0.24222^{\circ} \mathrm{S}, 100.35277^{\circ} \mathrm{E}$, 900 m, 2-3 June 2006, P. Schwendinger, 1 ㅇ (MHNG, PBI_OON 00012441); Lake Maninjau, waterfall above Gasang, $3.51666^{\circ} \mathrm{N}$, $98.35000^{\circ} \mathrm{E}, 720 \mathrm{~m}, 9$ Feb. 2000, P. Schwendinger, 9 s, 4 क (MHNG, PBI_OON 00012814); same location, 10 Feb. 2000, P. Schwendinger, 11 ๙े, 6 क (MHNG, 

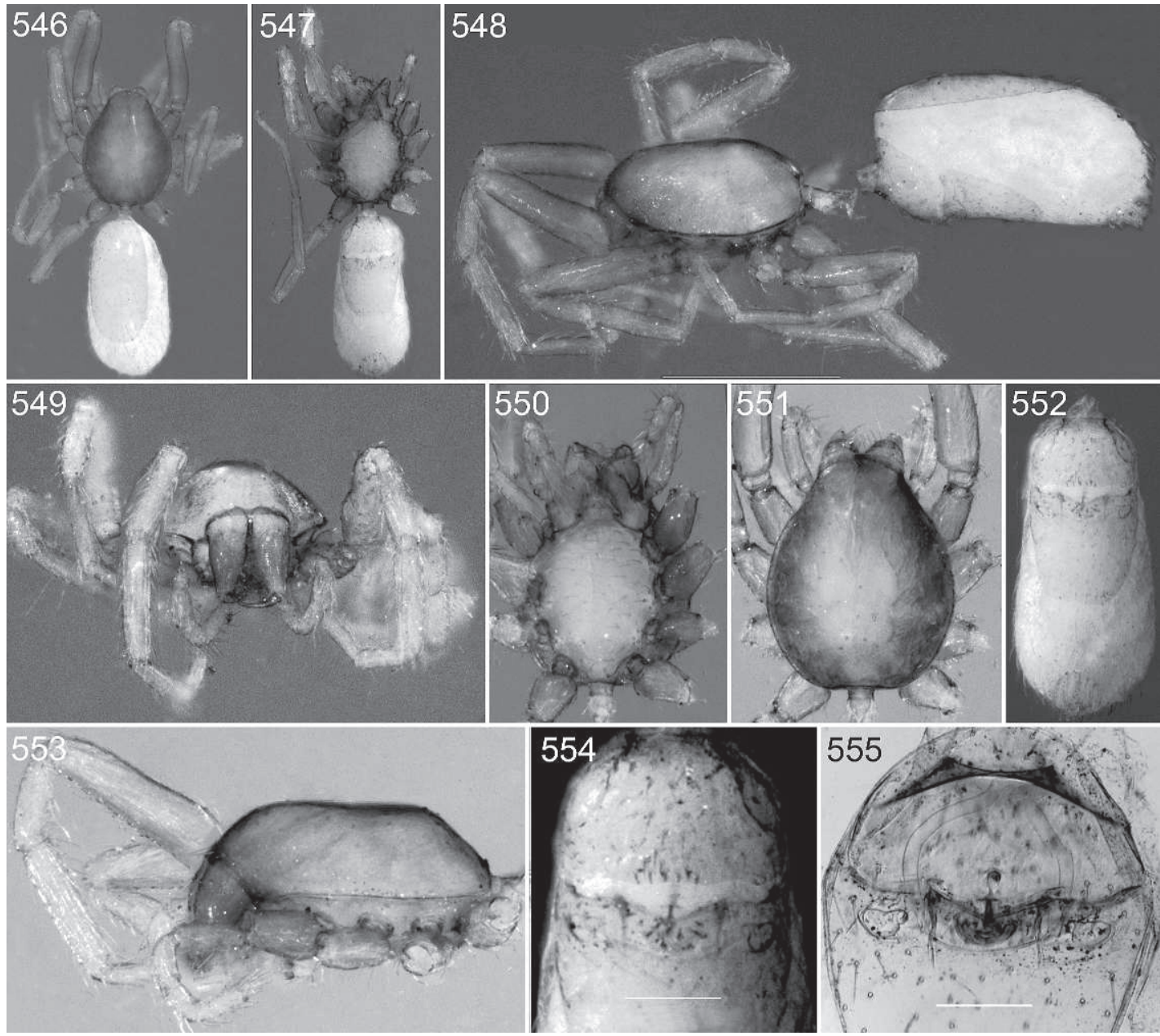

Figs. 546-555. Prethopalpus eberhardi, n. sp., female (PBI_OON 18854). 546. Habitus, dorsal view. 547. Same, ventral view. 548. Same, lateral view. 549. Prosoma, anterior view. 550. Same, ventral view. 551. Same, dorsal view. 552. Abdomen, ventral view. 553. Prosoma, lateral view. 554. Female epigyne, ventral view. 555. Female epigyne, dorsal view.

PBI_OON 00012853); Mt Merapi, ca. $15 \mathrm{~km}$ SE of Bukittinggi, $0.39222^{\circ} \mathrm{N}, 100.44833^{\circ} \mathrm{E}$, $2100 \mathrm{~m}, 4$ June 2006, P. Schwendinger, 1 s, 2 은 (MHNG, PBI_OON 00012438). Jambi: Mt. Kerinci, W of Kersik Tua, $1.53333^{\circ} \mathrm{S}$, 101.21666 ${ }^{\circ}$ E, 2,160 m, 17-18 Nov. 2000, P. Schwendinger, 2 क (MHNG, PBI_ OON 00012139); Mt. Kerinci, $1.91666^{\circ} \mathrm{S}$, $101.33333^{\circ} \mathrm{E}, 3,300 \mathrm{~m}, 12$ Nov. 1989, Agosti, Loebl, Burckhardt, 1 i (MHNG, PBI_OON 00012081); Mt. Tujuh [1.69000 $\left.{ }^{\circ} \mathrm{S}, 101.43000^{\circ} \mathrm{E}\right]$, $1,500 \mathrm{~m}, 20$ Feb. 2000, P. Schwendinger,
2 옹 (MHNG, PBI_OON 00012897). Lampung: Bukit Barisan Selatan National Park, on road from Krui to Liwa, $2 \mathrm{~km} \mathrm{SW}$ of Kubuprahu, $0.01666^{\circ} \mathrm{S}, 100.53333^{\circ} \mathrm{E}$, 480 m, 4 Mar. 2000, P. Schwendinger, 1 ઈ (MHNG, PBI_OON 00012379). Aceh: Gunung Leuser National Park, Ketambe [3.17193 $\left.{ }^{\circ} \mathrm{N}, 98.39077^{\circ} \mathrm{E}\right], 300 \mathrm{~m}, 23-30$ Nov. 1989, I. Löbl, D. Agosti and D. Burckhardt, 5 §̊, 3 ㅇ (MHNG, PBI_OON 00012078).

Distribution: Prethopalpus utara is known from Sumatra in Indonesia (map 4). 


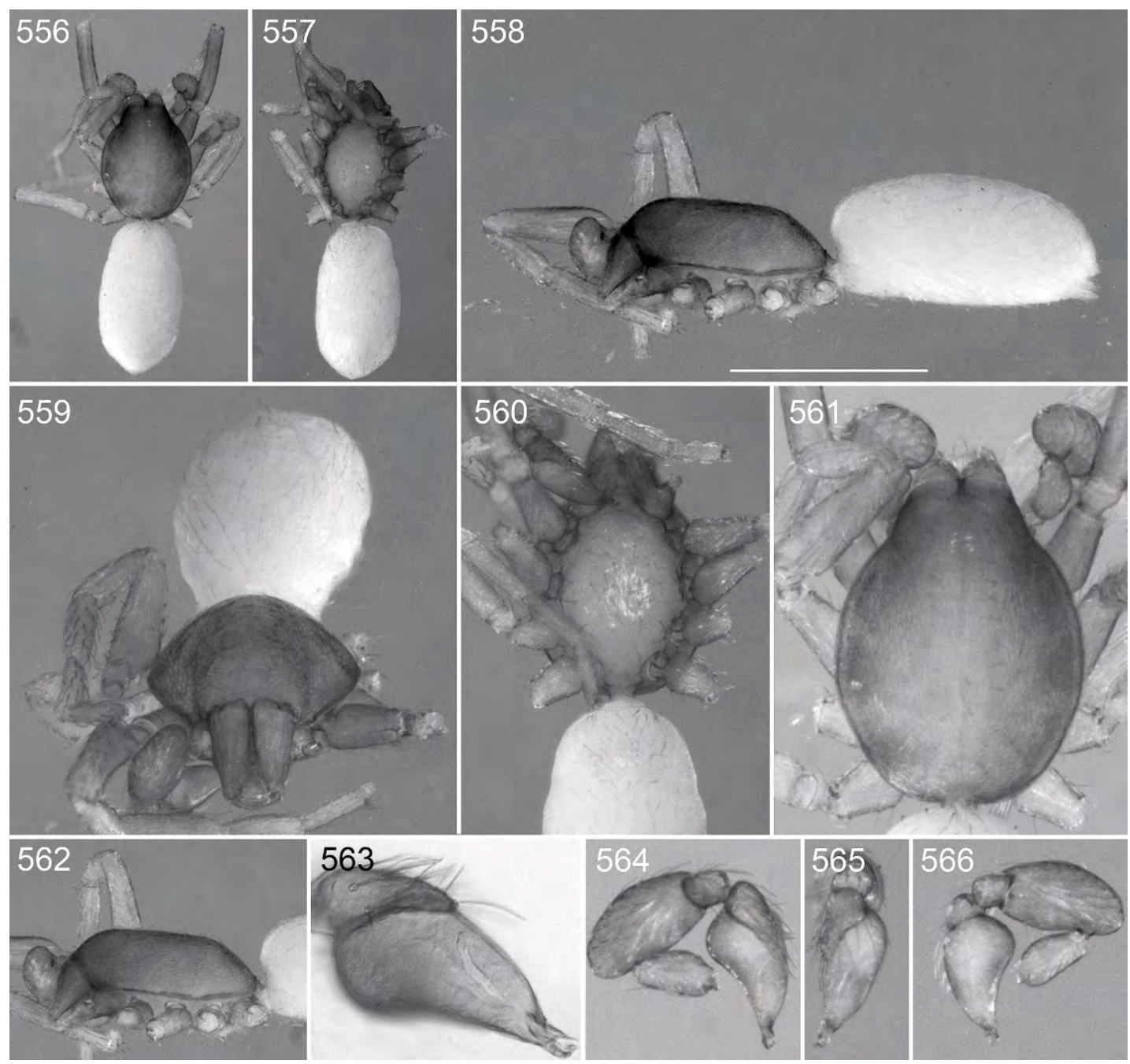

Figs. 556-566. Prethopalpus framenaui, n. sp., male (PBI_OON 46542). 556. Habitus, dorsal view. 557. Same, ventral view. 558. Same, lateral view. 559. Prosoma, anterior view. 560. Prosoma, ventral view. 561. Prosoma, dorsal view. 562. Prosoma, lateral view. 563. Male palpal bulb. 564. Male palp, prolateral view. 565. Same, dorsal view. 566. Male palp, retrolateral view.

\section{SPECIES FROM AUSTRALIA AND PAPUA NEW GUINEA}

\section{Key to Species}

1. Males.................... 2

- Females (unknown for $P$. alexanderi, $P$. attenboroughi, $P$. blosfeldsorum, $P$. oneillae, $P$. cooperi, $P$. infernalis and $P$. rawlinsoni) . . 20

2. Eyes absent or strongly reduced (e.g., figs. 492, 513, 561, 677) . . . . . . 3

- $\quad$ Eyes present, not strongly reduced (e.g., figs.

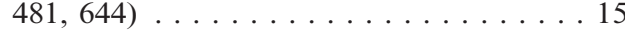

3. Eyes small (fig. 677)
- $\quad$ Eyes absent (e.g., figs. 492, 513, 561). . . 4

4. Inner margin of embolus smooth (fig. 117); clypeus without a lateral process (fig. 677) . . . . . . . . . . P. platnicki

- Inner margin of embolus undulate (fig. 114); clypeus with a lateral process (fig. 475) . . . . . . . ............ P. attenboroughi

5. Abdomen with dorsal scutum (e.g., figs. 548, $600) \ldots \ldots \ldots \ldots \ldots \ldots$

- Abdomen without dorsal scutum (e.g., figs. 576, 590) . . . . . . . . . . 13

6. Dorsal scutum covering almost the whole abdomen (e.g., figs. 458, 526) . . . . . . 7 


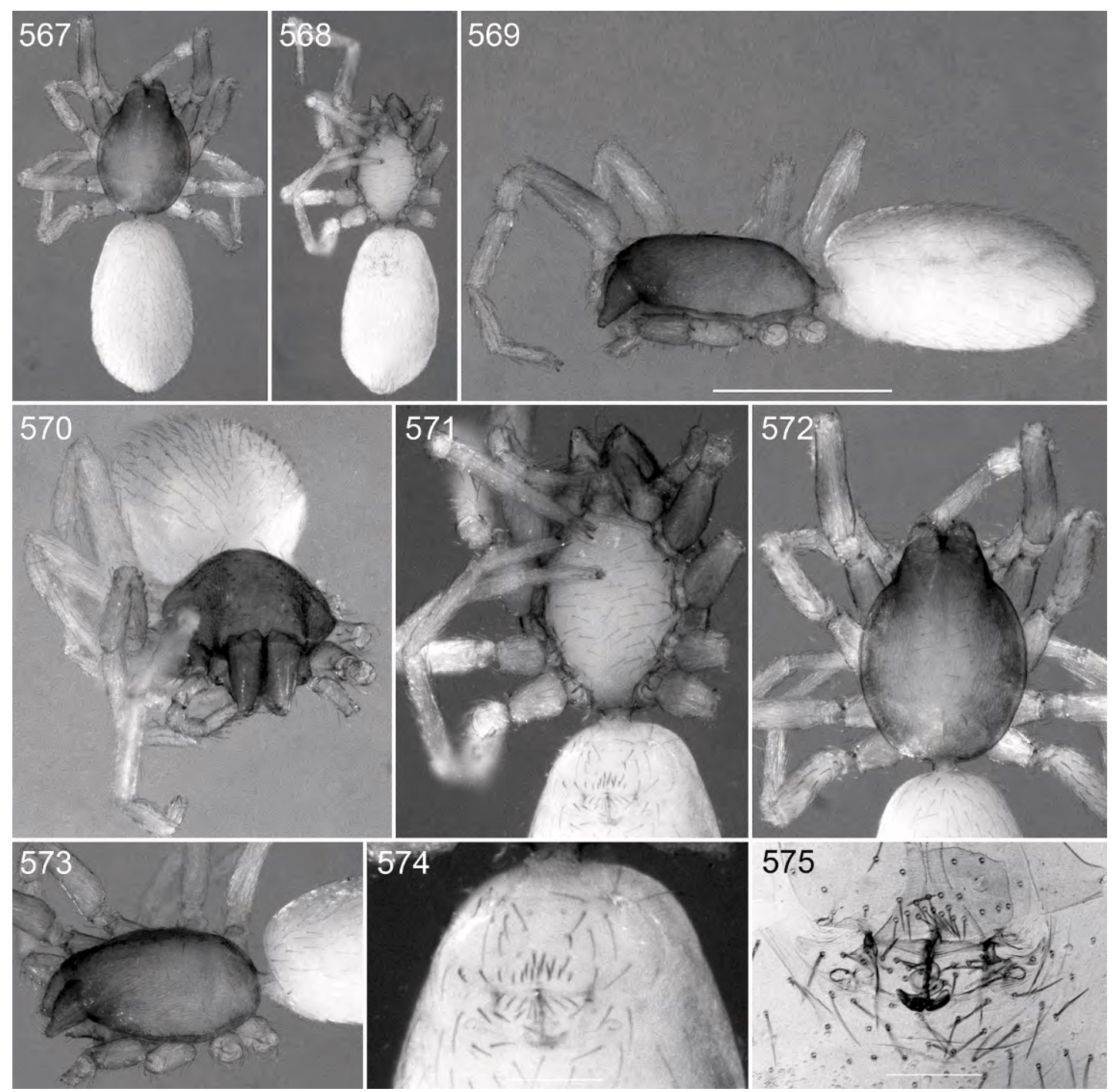

Figs. 567-575. Prethopalpus framenaui, n. sp., female (PBI_OON 23554). 567. Habitus, dorsal view. 568. Same, ventral view. 569. Same, lateral view. 570. Prosoma, anterior view. 571. Same, ventral view. 572. Same, dorsal view. 573. Same, lateral view. 574. Female epigyne, ventral view. 575. Female epigyne, dorsal view.

- Dorsal scutum covering about or less than $80 \%$ of abdomen (figs. 539, 600, 626) . . 11

7. Abdomen cylindrical (figs. 458, 526, 608, 691) . . . . . . . . . . . . . 8

- Abdomen ovoid (fig. 660) ..... P. oneillae

8. Distance between coxae II-III larger than others (fig. 695). . . . . . . . . P. pearsoni

- Distance between coxae about equal (figs. $462,530,612) \ldots \ldots \ldots \ldots$

9. Epigastric area strongly bulging in lateral view (fig. 538) . . . . . . . . P. cooperi
- Epigastric area flat in lateral view (figs. 460, $610) \ldots \ldots \ldots \ldots \ldots$

10. Endites about 1.5 times as long as wide (fig. 612); palpal patella posteriorly rounded (figs. 615, 617) ........ P. julianneae

- Endites at least twice as long as wide (fig. 462); palpal patella posteriorly pointed (figs. 467, 469) . . . . . . . P. alexanderi

11. Dorsal scutum covering about $70 \%$ of abdomen (fig. 600) . . . . . . . . . . P. infernalis

- Dorsal scutum covering about $80 \%$ of abdomen (figs. 539, 626) . . . . . . . . . . 12 
12. Palpal patella posteriorly pointed (figs. 543, 545) . . . . . . . . . . . . P. eberhardi Palpal patella posteriorly rounded (figs. 633, 635) .............. P. maini

13. Book lung covers darker than surrounding area (fig. 589) . . . . . . . . . . P. humphreysi Book lung covers pale, like surrounding area (figs. 494, 512, 560, 713) . . . . . . . 14

14. Carapace weakly sclerotized (fig. 492); palpal patella posteriorly pointed (figs. 496, 498). . .

P. boltoni

Carapace well sclerotized (figs. 513, 561, 714); palpal patella posteriorly rounded (e.g., figs. 515, 564, 716) . . . . . . 15

15. Chelicerae frontally swollen (fig. 514). . . . . . . . . ............... callani Chelicerae frontally not swollen (figs. 562, 715) . . . . . . . . . . . 16

16. Epigastric area strongly bulging in lateral view (fig. 711)........... . scanloni Epigastric area flat in lateral view (fig. 558) . . . . . . . . . P. framenaui

17. Bulb ventrally bulging (e.g., figs. 119, 122, 487, 705); epigastric region with strongly protruding extension (figs. 483, 704) . . . 18 Bulb not ventrally bulging (e.g., figs. 477, $649,738)$; epigastric region without protruding extension (figs. 472, 646, 733) . . . . . 19

18. Bulb with conical extension (figs. 122, 124, 705, 708) .......... P. rawlinsoni Bulb with rounded extension (figs. 119, 121, 487, 489) . . . . . . . . P. blosfeldsorum

19. Sternum surface finely reticulate (figs. 720 , 735) . . . . . . . . . . P. tropicus Sternum surface smooth (fig. 648) . . . . . . . . . ............ P. marionae

20. Eyes absent or strongly reduced (e.g., figs. $504,523,687) \ldots \ldots \ldots \ldots 21$ Eyes present, not strongly reduced (e.g., figs. 657, 746) . . . . . . . . . . . . . . 29

21. Eyes small (fig. 687) . . . . . . P. platnicki Eyes absent (e.g., figs. 504, 523) . . . . . . 22

22. Abdomen with dorsal scutum (figs. 546, 636) . . . . . . . . . . . . . 23 Abdomen without dorsal scutum (e.g., figs. 499, 590, 618) . . . . . . . . . 24

23. Carapace broadly oval (fig. 551) ... P. eberhardi Carapace long oval (fig. 641) ... . P. maini

24. Distance between coxae II-III larger than others (fig. 622). . . . . . . . . . P. kintyre Distance between coxae about equal (figs. $503,522,571,594) \ldots \ldots 25$

25. Book lung covers much darker than surrounding abdominal region (fig. 598). . . . . . . P. humphreysi Book lung covers pale,same color as surrounding abdominal region (figs. 505, 524, $558) \ldots \ldots \ldots \ldots 26$
26. Carapace weakly sclerotized (fig. 504) ..... . ............. P. boltoni Carapace well sclerotized (fig. 523, 572) . . 28

28. Internal genital structure reaching groove of posterior spiracles (fig. 575) . . . P. framenaui Internal genital structure not reaching groove of posterior spiracles (fig. 525) ... P. callani

29. Internal genital structure reaching beyond tracheal groove (figs. 157, 158). . . . P. marionae Internal genital structure shorter, not reaching beyond tracheal groove (fig. 156). . . . . .

$$
\text { P. tropicus }
$$

\section{Prethopalpus alexanderi Baehr and Harvey, new species}

Figures 458-469; map 10

TyPe: AUSTRALIA: Western Australia: Male holotype from Exmouth Limestone, $9.4 \mathrm{~km} \mathrm{~S}$ of Exmouth, bore, $-20 \mathrm{~m}$, $22^{\circ} 00^{\prime} 03^{\prime \prime} \mathrm{S}, 114^{\circ} 04^{\prime} 47^{\prime \prime} \mathrm{E}$ (25 July 2008, J. Alexander, J. Cairnes), deposited in WAM (T95473, PBI_OON 46551).

ETYMOLOGY: This species is named for Jason Alexander, one of the collectors of the holotype.

Diagnosis: Males resemble those of $P$. oneillae and $P$. cooperi in having a dorsal scutum covering the whole abdomen and no eyes, but can be separated from these species by the cylindrical abdomen (fig. 458) and the flat epigastric area (when viewed laterally) (fig. 460).

MAle (PBI_OON 46551, figs. 458-469): Total length 1.05. Carapace, sternum, mouthparts, palp, legs, and scuta pale orange, ovoid in dorsal view, pars cephalica flat in lateral view, surface smooth, sides slightly granulate; Clypeus straight in front view, vertical in lateral view. Sternum fused to carapace, surface smooth. Endites twice as long as wide. Abdomen cylindrical; book lung covers large. Dorsal scutum weakly sclerotized, without color pattern, covering full length of abdomen, no soft tissue visible from above, middle surface smooth, sides smooth; postepigastric scutum weakly sclerotized, long, almost rectangular, covering nearly full length of abdominal length, without posteriorly directed lateral apodemes; spinneret scutum with fringe of stout setae. Legs: patella plus tibia I shorter than carapace. Genitalia: epigastric region with 


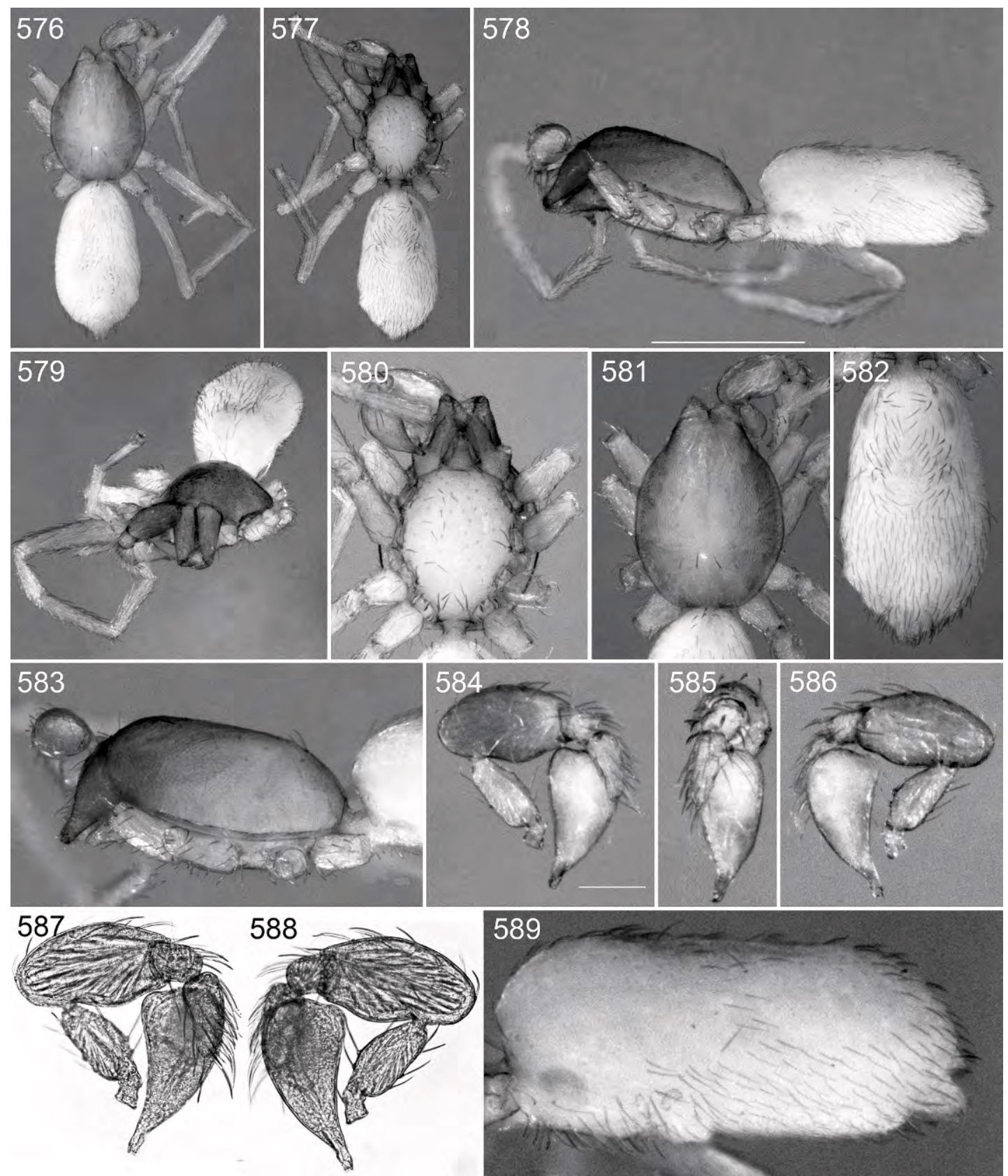

Figs. 576-589. Prethopalpus humphreysi, n. sp., male (PBI_OON 46538). 576. Habitus, dorsal view. 577. Same, ventral view. 578. Same, lateral view. 579. Prosoma, anterior view. 580. Same, ventral view. 581. Same, dorsal view. 582. Abdomen, ventral view. 583. Prosoma, lateral view. 584. Male palp, prolateral view. 585. Same, dorsal view. 586. Same, retrolateral view. 587. Same, prolateral view. 588. Same, retrolateral view. 589. Abdomen, lateral view. 


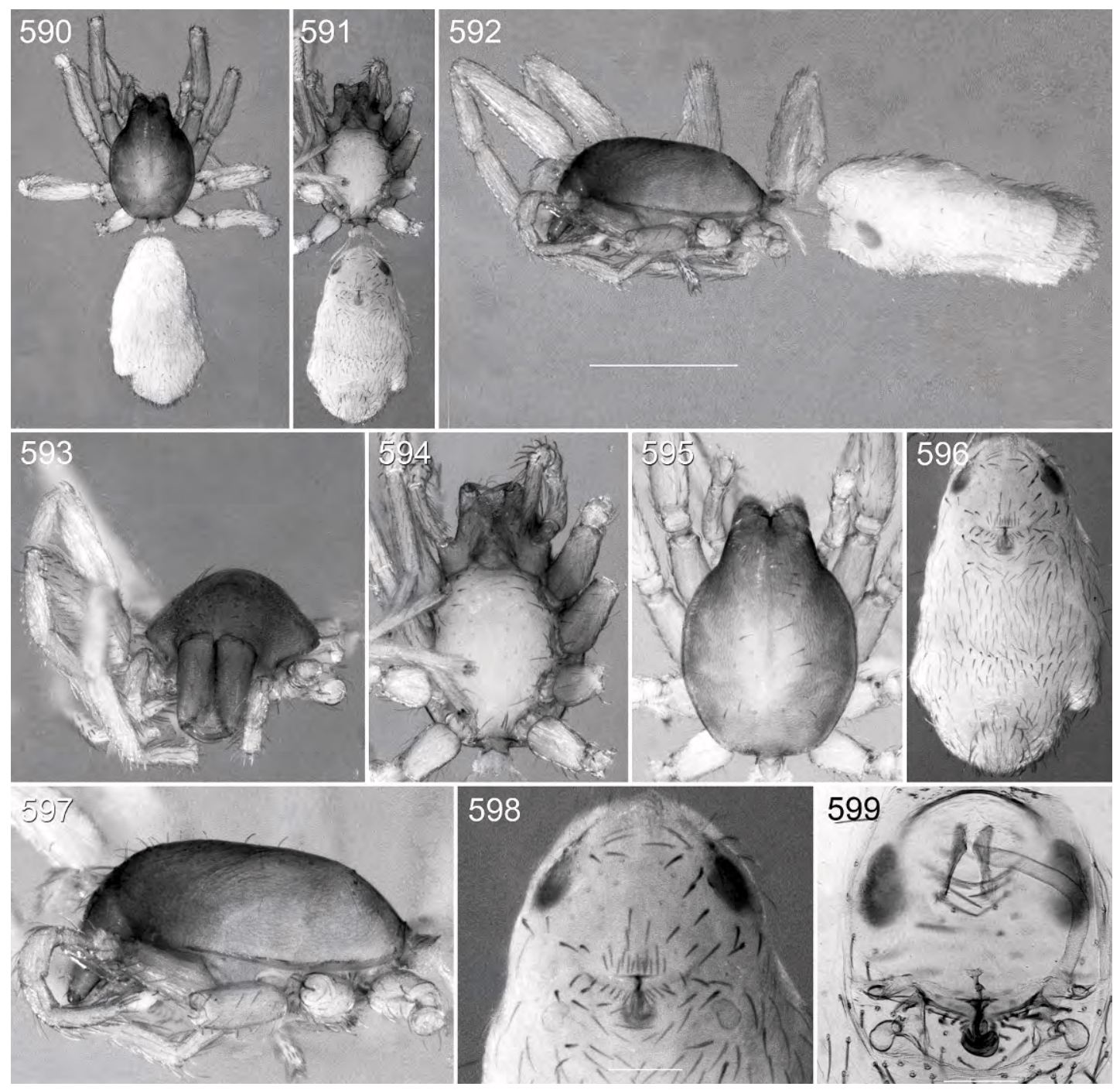

Figs. 590-599. Prethopalpus humphreysi, n. sp., female (PBI_OON 23555). 590. Habitus, dorsal view. 591. Same, ventral view. 592. Same, lateral view. 593. Prosoma, anterior view. 594. Same, ventral view. 595. Same, dorsal view. 596. Abdomen, ventral view. 597. Prosoma, lateral view. 598. Female epigyne, ventral view. 599. Female epigyne, dorsal view.

sperm pore small, circular, situated in front of anterior spiracles. Palpal patella slightly bulging ventrally with conical base (fig. 467); bulb pear shaped with narrow curled embolar tip (figs. 466-469).

FEMALE: Unknown.

Other Material Examined: None.

DISTRIBUTION: This species is known only from a borehole south of Exmouth, Cape Range, northwestern Australia (map 7). Al- though this specimen came from a location near the type locality of $P$. infernalis, they undoubtedly represent different species.

Prethopalpus attenboroughi Baehr and Harvey, new species

Figures 113-115, 470-480; map 7

TyPe: AUSTRALIA: Queensland: Male holotype from Horn Island, Torres Strait, $10.61667^{\circ} \mathrm{S}, 142.2833^{\circ} \mathrm{E}(2-8$ Dec. 1986, J. 


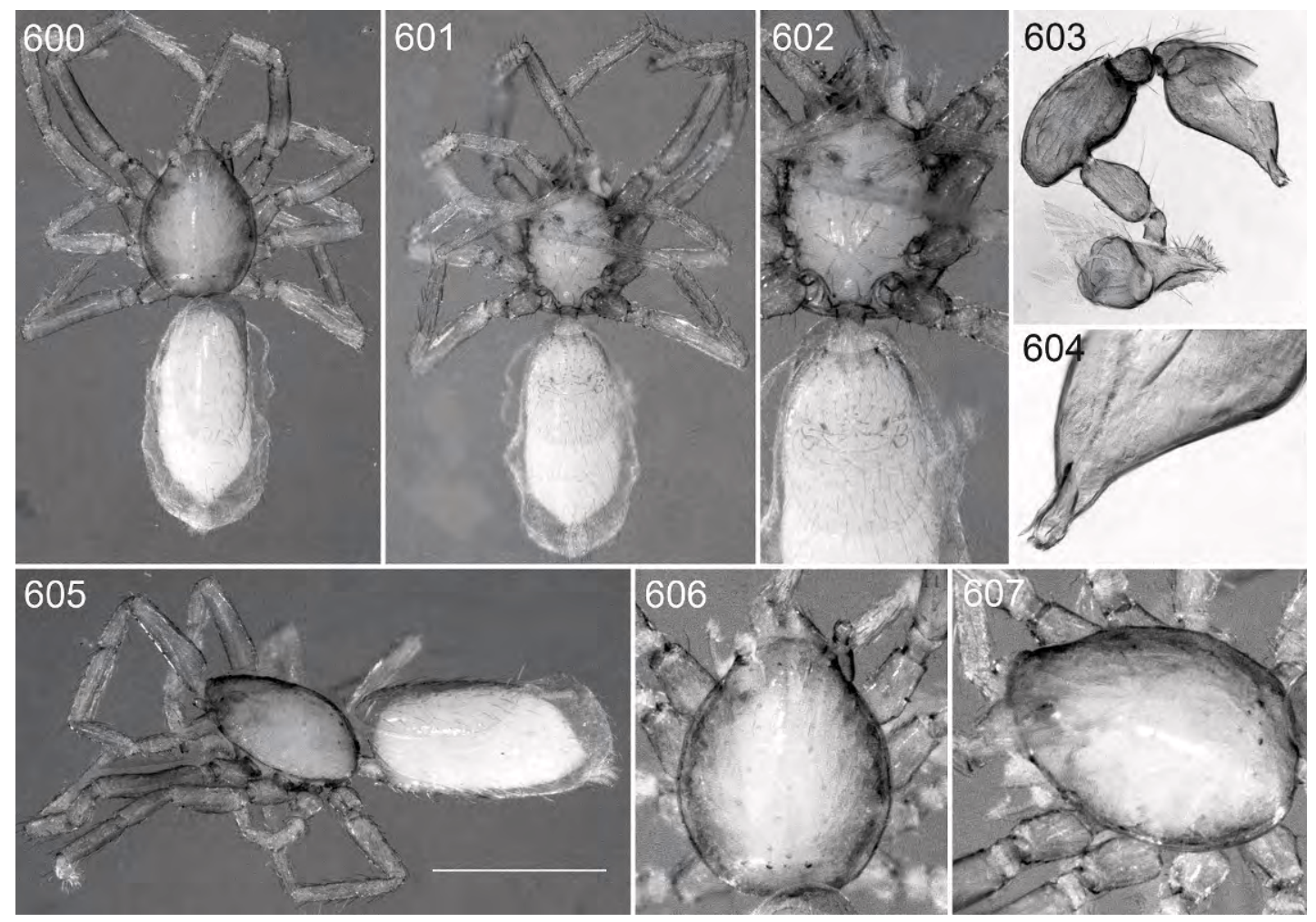

Figs. 600-607. Prethopalpus infernalis (Harvey and Edward), male (PBI_OON 46550). 600. Habitus, dorsal view. 601. Same, ventral view. 602. Prosoma, anterior portion of abdomen, ventral view. 603. Male palp, prolateral view. 604. Same, detail of bulb, retrolateral view. 605. Habitus, lateral view. 606. Prosoma, dorsal view. 607. Prosoma, dorsolateral view.

Gallon), deposited in QM (S72955, PBI_OON 00007438).

ETYMOLOGY: This species is named for the eminent naturalist David Attenborough in honor of his contributions to recognizing the beauty and splendor of the world's biodiversity.

DiagnOSIs: Males resemble those of $P$. platnicki in general body shape and bulbal shape, but can be separated by the larger eyes (fig. 473), and a lateral process on the clypeus (fig. 475).

MALE (PBI_OON 7438, figs. 470-480): Total length 1.04. Prosoma, mouthparts, palp, legs, and abdominal scuta white, surface of elevated portion of pars cephalica smooth, sides granulate, clypeus with pair of small projections containing stiff setae; lateral margin without denticles. Eyes: six, tiny; ALE 0.030, PME 0.016, PLE 0.016; ALE largest, all eyes circular; posterior eye row recurved from above, straight from front; ALE separated by more than their diameter, ALE-PLE separated by less than ALE radius, $\mathrm{PME}$ separated by their radius to diameter, PLE-PME separated by less than PME radius. Endites twice as long as wide, with tiny toothlike projection. Abdomen cylindrical; book lung covers large; pedicel tube short, ribbed. Dorsal scutum covering full length of abdomen smooth; epigastric scutum not protruding; postepigastric scutum long, almost rectangular, covering about $3 / 4$ of abdomen, without posteriorly directed lateral apodemes. Legs: patella plus tibia I nearly as long as carapace. Epigastric region with circular sperm pore. Palpal bulb, distal tip with broad a medially bent hook (figs. 113-115).

FEMALE: Unknown.

Other MATERIAl ExAMINED: AUSTRALIA: Queensland: Horn Island, Torres Strait, 


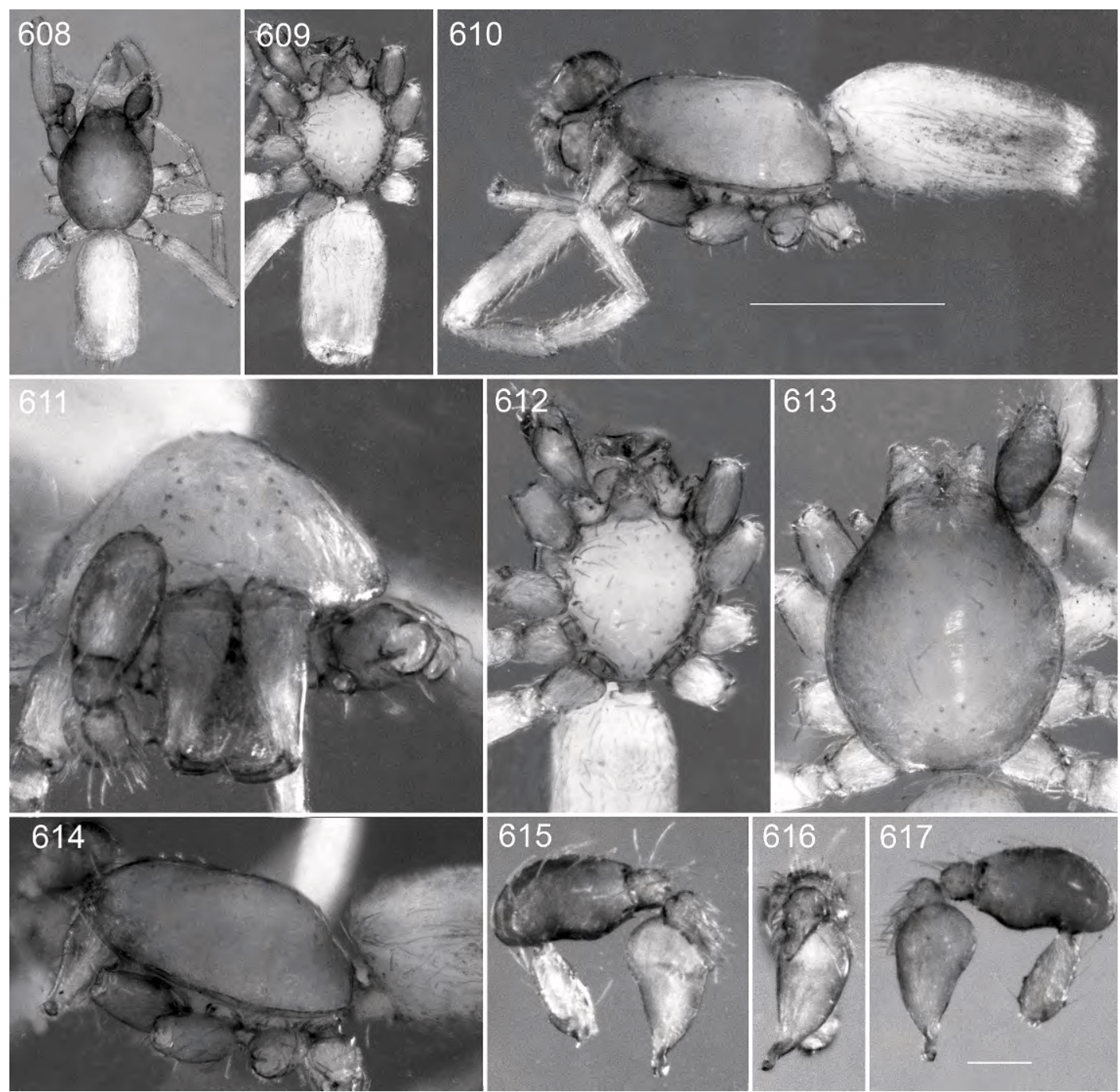

Figs. 608-617. Prethopalpus julianneae, n. sp., male (PBI_OON 46587). 608. Habitus, dorsal view. 609. Same, ventral view. 610. Same, lateral view. 611. Prosoma, anterior view. 612. Same, ventral view. 613. Same, dorsal view. 614. Same, lateral view. 615. Male palp, prolateral view. 616. Same, dorsal view. 617. Palp, retrolateral view.

$10.61667^{\circ} \mathrm{S}, 1^{142.2833^{\circ} \mathrm{E},} 2-8$ Dec. 1986, J. Gallon, 1 o (QM S72955, PBI_OON 00007438).

DISTRIBUTION: This species is known only from the type locality, Horn Island in the Torres Strait (map 7).

Prethopalpus blosfeldsorum Baehr and Harvey, new species

Figures 119-121, 481-489; map 7

TyPe: AUSTRALIA: Queensland: Male holotype from Kroombit Tops, 45 km SSW of Calliope (site 2), $24.40611^{\circ} \mathrm{S}, 150.98333^{\circ} \mathrm{E}$, 860 m (15 Dec. 1983, G. Monteith, V. Davies, J. Gallon, G. Thompson), deposited in QM (S12800, PBI_OON 00006425).

ETYMOLOGY: The specific name honors Bruno and Denise Blosfelds, M.S.H.'s parents-in-law.

Diagnosis: Males resemble those of $P$. rawlinsoni in having a strongly protruding epigastric scutum and a ventrally bulging bulb, but can be separated by having a bulb 


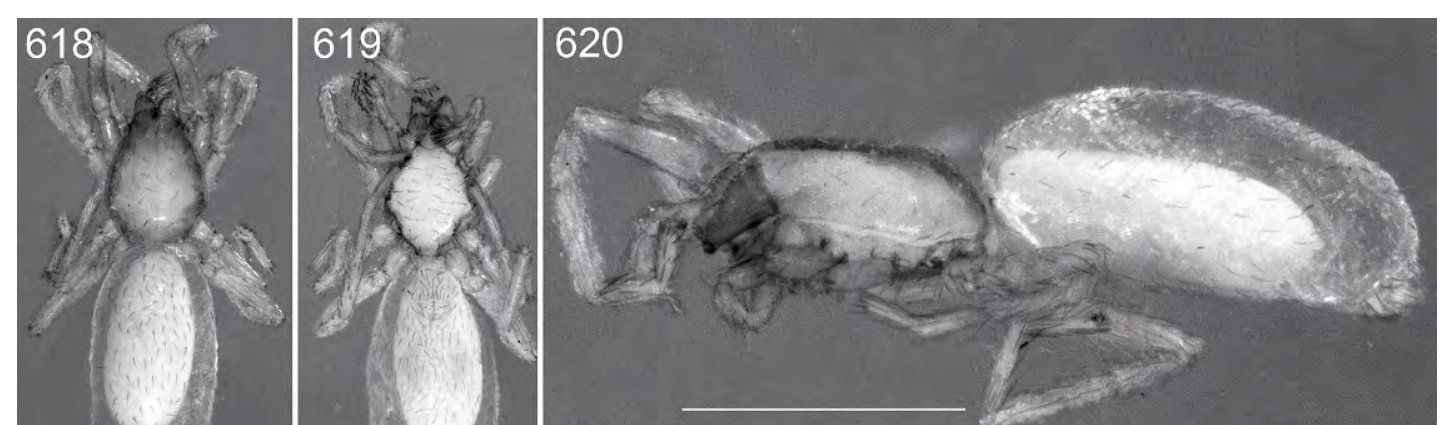

Figs. 618-625. Prethopalpus kintyre, n. sp., female (PBI_OON 46574). 618. Habitus, dorsal view. 619. Same, ventral view. 620. Same, lateral view. 621. Prosoma, anterior view. 622. Same, ventral view. 623. Same, dorsal view. 624. Same, lateral view. 625. Female epigyne, ventral view.

with a rounded basal extension (figs. 119, 121)

Male (PBI_OON 6425, figs. 481-489): Total length 1.05. Prosoma, mouthparts, and abdominal scuta yellow-brown, pars cephalica flat in lateral view, surface of elevated portion of pars cephalica smooth, sides finely reticulate; lateral margin without denticles; nonmarginal pars cephalica setae light, needlelike, present, scattered; marginal setae light, needlelike. Clypeus margin unmodified, straight in front view, vertical in lateral view, 


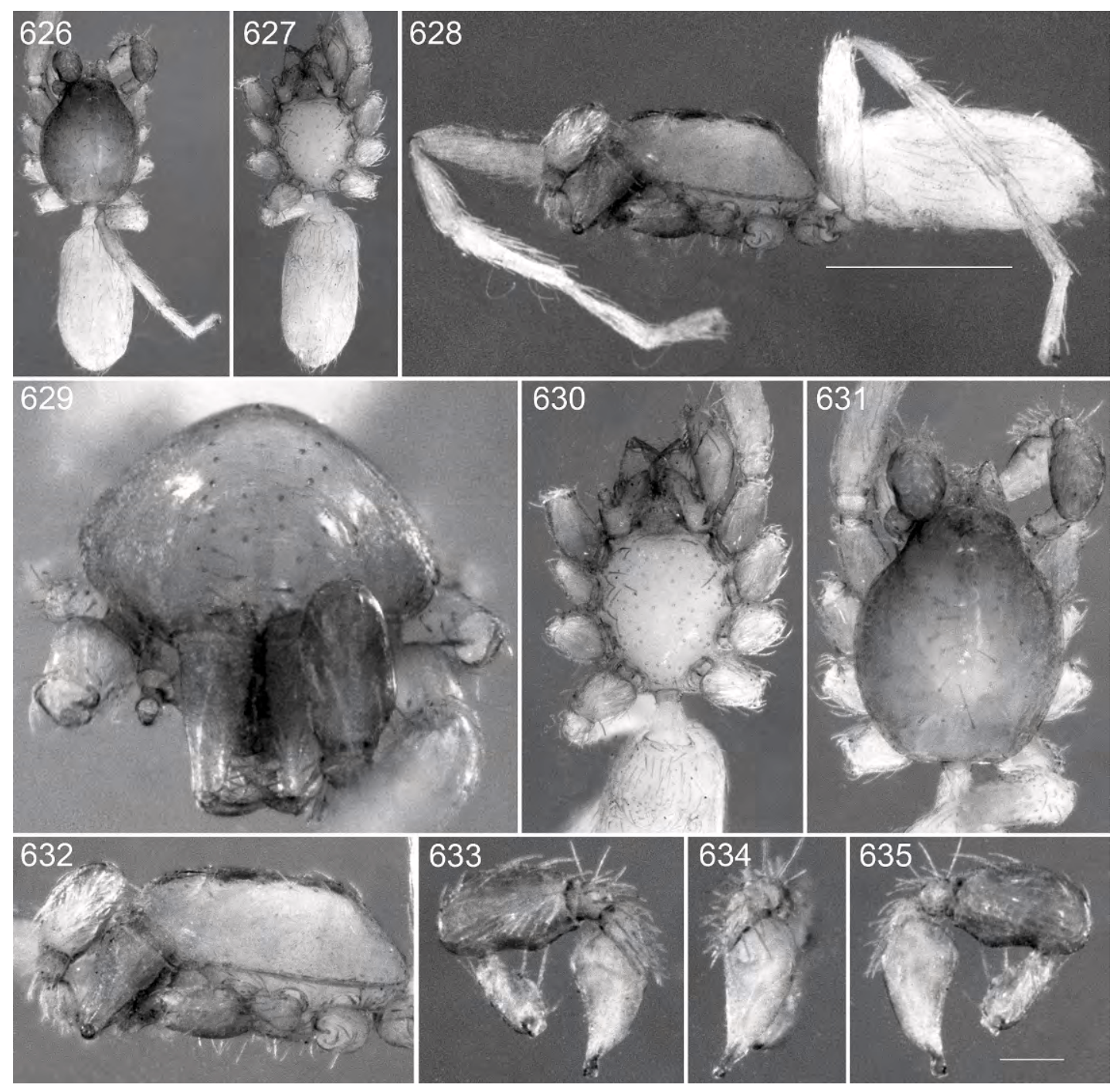

Figs. 626-635. Prethopalpus maini, n. sp., male (PBI_OON 46586). 626. Habitus, dorsal view. 627. Same, ventral view. 628. Same, lateral view. 629. Prosoma, anterior view. 630. Same, ventral view. 631. Same, dorsal view. 632. Same, lateral view. 633. Male palp, prolateral view. 634. Same, dorsal view. 635. Palp, retrolateral view.

high, ALE separated from edge of carapace by their radius or more; setae present, light, needlelike. Eyes: ALE 0.040, PME 0.039, PLE 0.038; ALE largest, all eyes circular; posterior eye row straight from both above and front; ALE separated by less than their radius, ALE-PLE separated by less than ALE radius, PME just touching, PLE-PME separated by less than PME radius. Sternum longer than wide, surface smooth, without pits. Endites twice as long as wide, toothlike projection tiny. Abdomen cylindrical; book lung covers small; pedicel tube short, ribbed. Dorsal scutum covering full length of abdomen, smooth; epigastric scutum strongly protruding; postepigastric scutum long, almost rectangular, covering about $3 / 4$ of abdomen, without posteriorly directed lateral apodemes. Legs white; patella plus tibia I shorter than carapace. Epigastric region with circular sperm pore and strongly protruding extension. Palp yellow; embolus light; femur 


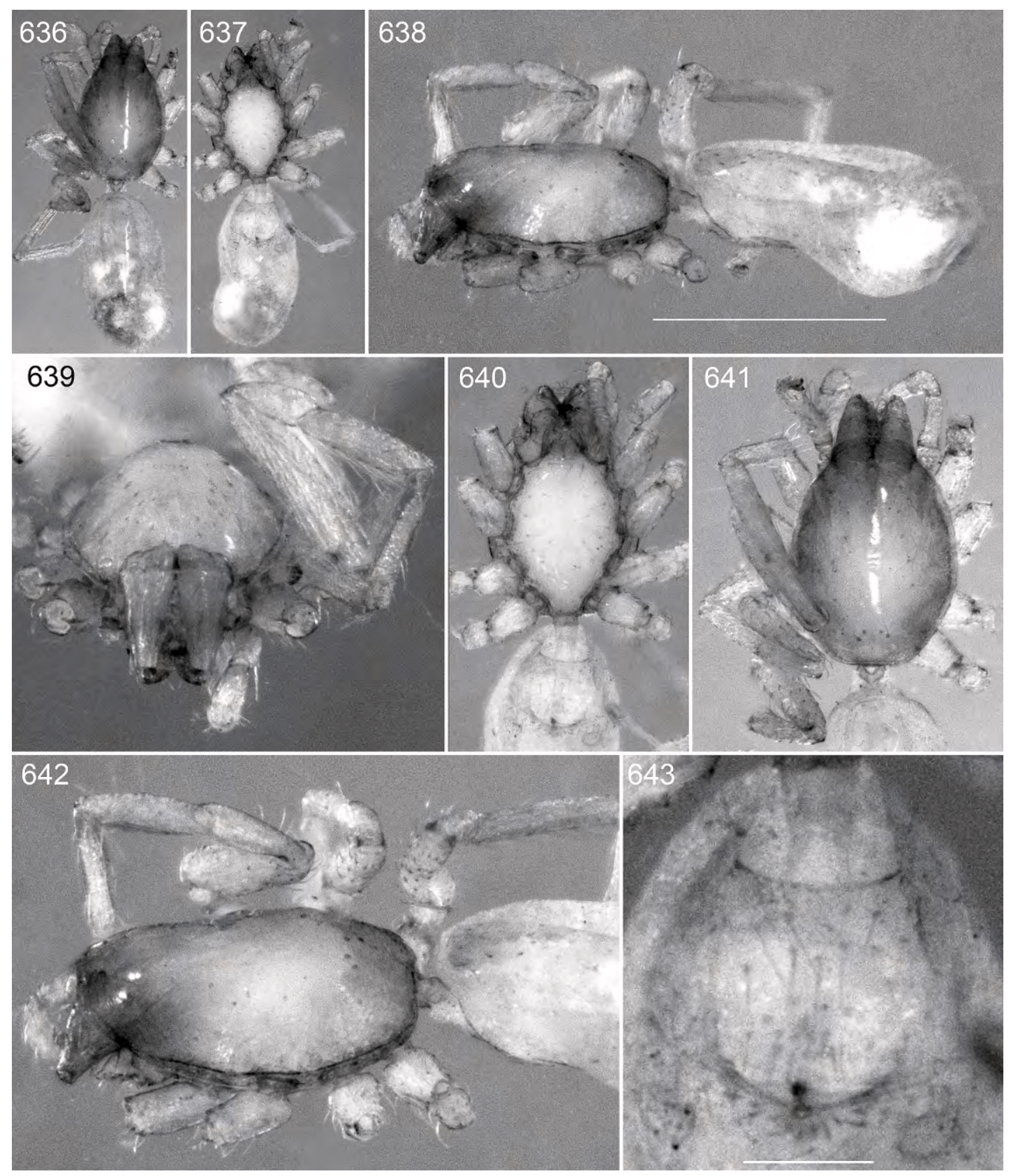

Figs. 636-643. Prethopalpus maini, n. sp., female (PBI_OON 46590). 636. Habitus, dorsal view. 637. Same, ventral view. 638. Same, lateral view. 639. Prosoma, anterior view. 640. Same, ventral view. 641. Same, dorsal view. 642. Same, lateral view. 643. Female epigyne, ventral view. 


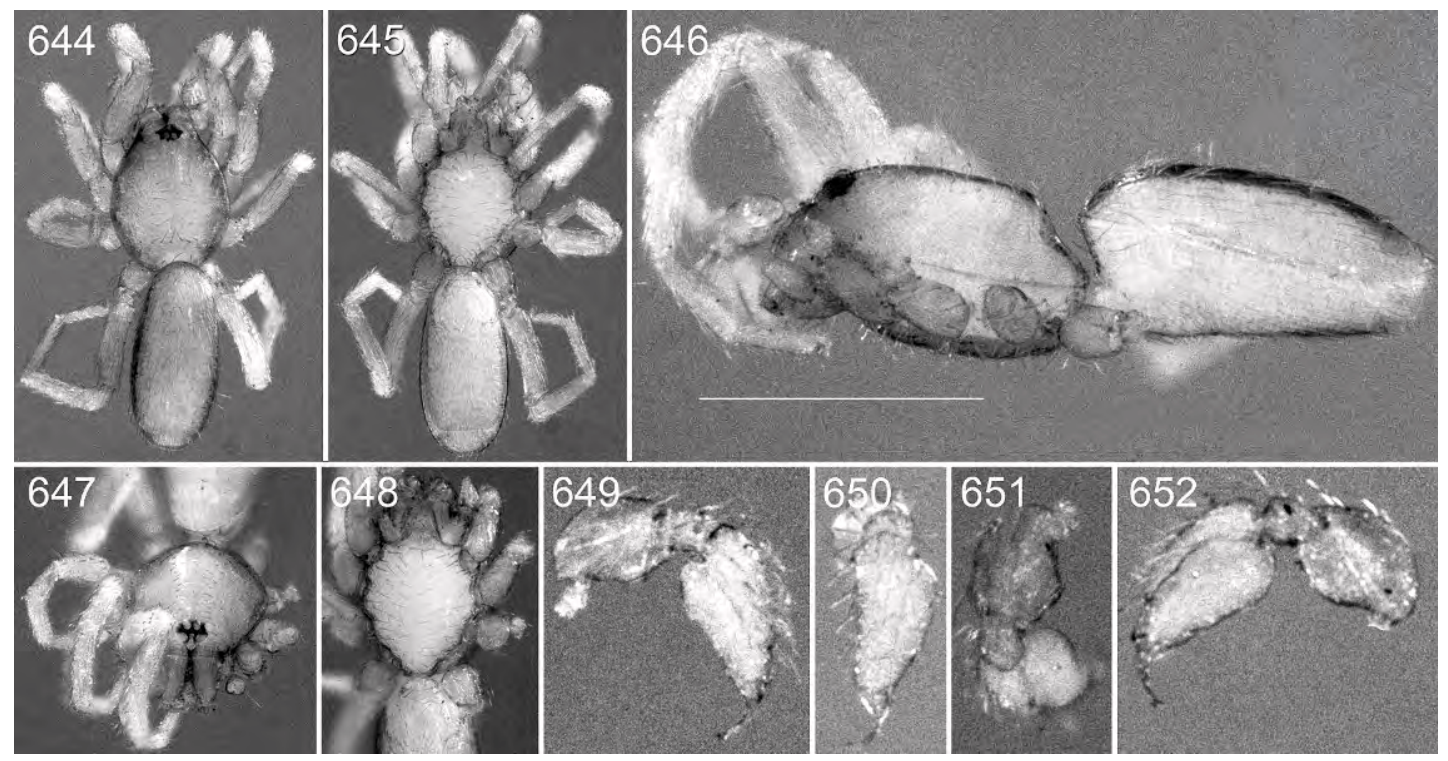

Figs. 644-652. $\quad$ Prethopalpus marionae, n. sp., male (PBI_OON 23006). 644. Habitus, dorsal view. 645. Same, ventral view. 646. Same, lateral view. 647. Prosoma, anterior view. 648. Same, ventral view. 649. Male palp, prolateral view. 650. Same, dorsal view. 651. Patella, dorsal view. 652. Palp, retrolateral view.

attaching to patella submedially; patella longer than femur; cymbium not fused with bulb, bulb ventrally strongly bulging with rounded extension (figs. 119-121).

FEMALE: Unknown.

Other MAterial Examined: AUSTRALIA: Queensland: Kroombit Tops, 45 km SSW of Calliope (site 2), $24.40611^{\circ} \mathrm{S}$, $150.98333^{\circ} \mathrm{E}, 860 \mathrm{~m}, 15$ Dec. 1983 (G. Monteith, V. Davies, J. Gallon, G. Thompson, QM S80973, PBI_OON 00022766, QM S16187, PBI_OON 00022767, QM S12829, PBI_OON 00022769), 4 s.

DisTRIBUTION: This species is known only from the type locality in eastern Queensland (map 7).

Prethopalpus boltoni Baehr and Harvey, new species

Figures 490-507; map 10

Types: AUSTRALIA: Western Australia: Male holotype from Hope Downs, $35 \mathrm{~km}$ NW of Newman, $23^{\circ} 07^{\prime} 52^{\prime \prime} \mathrm{S}, 119^{\circ} 32^{\prime} 01^{\prime \prime} \mathrm{E}$, stygofauna haul net (16-18 Feb. 2009, P. Bolton, J. Puglisi) (WAM T96817, PBI_OON 46552).
ETYMOLOGY: This species is named for Paul Bolton, one of the collectors of the type specimens.

DiAGNOSIS: Males and females resemble those of $P$. framenaui and $P$. humphreysi in lacking abdominal dorsal scutum and eyes, but can be easily separated by their much less sclerotized carapace (fig. 492). Males can be easily separated by the pointed basal region of the palpal patella (from lateral view) (figs. 496, 498), and females can be recognized by the long, straight posterior tube in the genitalia, and the short sinuous section, not reaching the posterior half between the epigastric and spiracular grooves (fig. 507).

Male (PBI_OON 46552, figs. 490-498): Total length 1.18. Prosoma, mouthparts, and abdominal scuta yellow, carapace ovoid in dorsal view, pars cephalica flat in lateral view, surface smooth. Sternum finely punctate. Endites twice as long as wide, directed medially. Abdomen ovoid; book lung covers not visible (abdomen in poor condition). Dorsal scutum absent; postepigastric scutum weakly sclerotized, almost semicircular, only around epigastric furrow, fused to epigastric scutum, without posteriorly directed lateral 


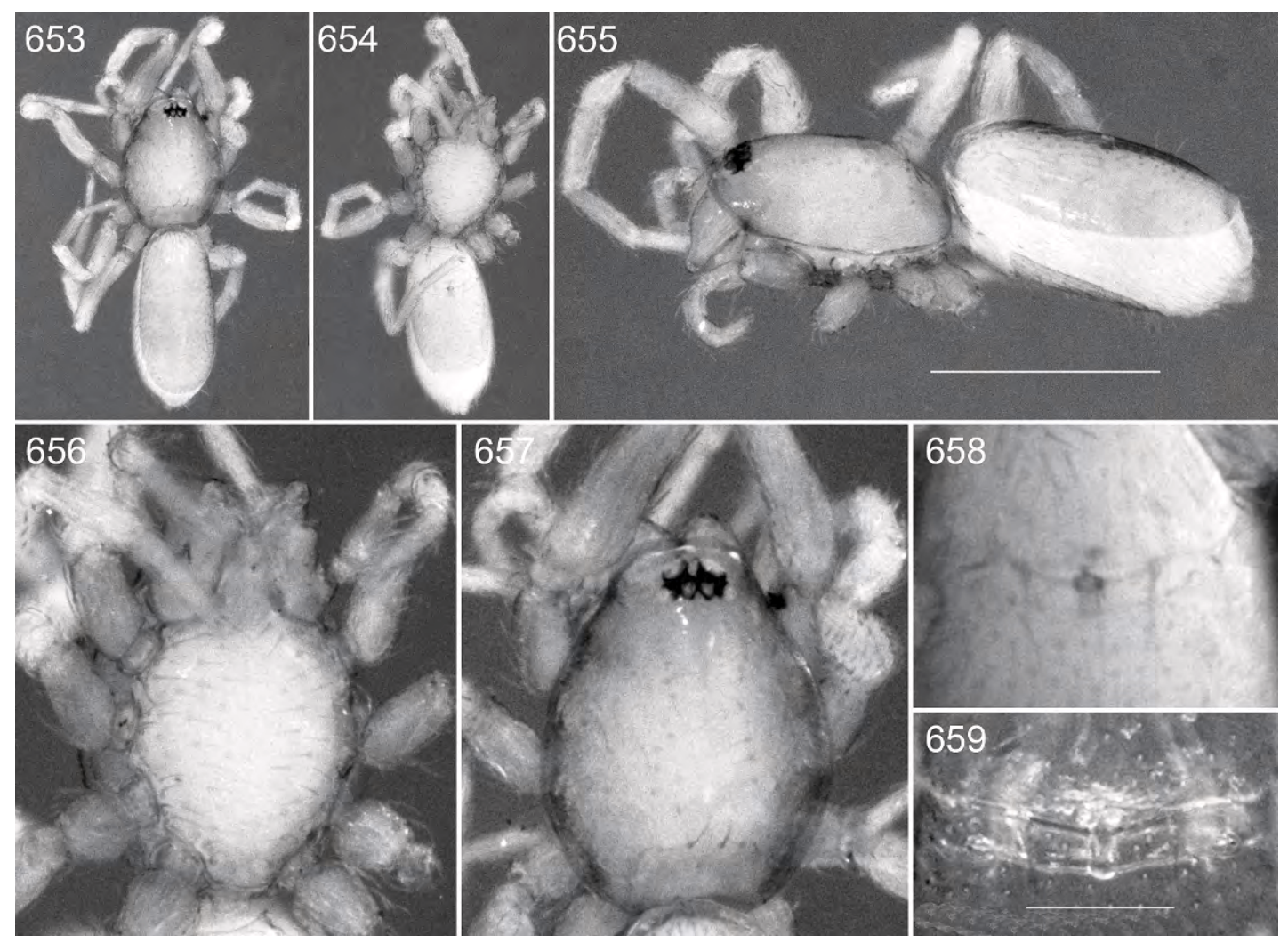

Figs. 653-659. Prethopalpus marionae, n. sp., female (PBI_OON 23403). 653. Habitus, dorsal view. 654. Same, ventral view. 655. Same, lateral view. 656. Prosoma, ventral view. 657. Same, dorsal view. 658. Female epigyne, ventral view. 659. Female epigyne, dorsal view.

apodemes; spinneret scutum not visible (abdomen in poor condition). Legs white; patella plus tibia I nearly as long as carapace. Genitalia: epigastric region with sperm pore small, circular, situated in front of anterior spiracles. Palp patella slightly bulging ventrally, with conical base; middle part of bulb only slightly bulging ventrally, with narrow curled embolar tip (figs. 496-498).

FeMAle (PBI_OON 18856, figs. 499507): Total length 1.29. Book lung covers and spinneret scutum not visible (abdomen in poor condition). Genitalia: epigastric area with broad slitlike epigynal opening, internal genital structure with free, needlelike anterior sclerite, and a long sinuous posterior tube, posterior part reaching half the distance to groove of posterior spiracles (fig. 507).

Other MATERIAl EXAmined: AUSTRALIA: Western Australia: Hope Downs, 35 km
NW of Newman, $23^{\circ} 07^{\prime} 51^{\prime \prime} \mathrm{S}, 119^{\circ} 32^{\prime} 00^{\prime \prime} \mathrm{E}$, stygofauna haul net, 13 Mar. 2008 (P. Bolton, C. Weston, WAM T92116, PBI_OON 18856), 1 o (now lost, see REMARKs).

DisTRIBUTION: This species is known only from a subterranean ecosystem at Hope Downs, situated in the Pilbara region of Western Australia (map 10).

REMARKS: Unfortunately, after the description of the female specimen was prepared, the specimen was lost.

Prethopalpus callani Baehr and Harvey, new species

Figures 508-525; map 10

Types: AUSTRALIA: Western Australia: Male holotype from Yeelirrie Station, $87 \mathrm{~km} \mathrm{~S}$ of Wiluna, bore YYHC0048H, 27 $18^{\prime} 59.90^{\prime \prime} \mathrm{S}$, $120^{\circ} 09^{\prime} 02.14^{\prime \prime} \mathrm{E}$, troglofauna (17 Mar. 2010, S. Callan, G. Perina), deposited in WAM 
Figs. 660-671. Prethopalpus oneillae, n. sp., male (PBI_OON 46537). 660. Habitus, dorsal view. 661. Same, ventral view. 662. Same, lateral view. 663. Prosoma, anterior view. 664. Prosoma, ventral view. 665. Prosoma, dorsal view. 666. Abdomen, ventral view. 667. Prosoma, lateral view. 668. Abdomen, anterior view. 669. Male palp, prolateral view. 670. Same, dorsal view. 671. Same, retrolateral view.

(T108171, PBI_OON 46576). Paratypes: 1 female, from Yeelirrie Station, $87 \mathrm{~km} \mathrm{~S}$ of Wiluna, bore YYHC0048H, 27 $18^{\prime} 59.90^{\prime \prime} \mathrm{S}$, $120^{\circ} 09^{\prime} 02.14^{\prime \prime} \mathrm{E}$, troglofauna (13 Nov. 2011, P. Bell, S. Callan), deposited in WAM (T108166, PBI_OON 46564); 1 female from Yeelirrie, $87 \mathrm{~km} \mathrm{~S}$ of Wiluna, bore YYAC285, $27^{\circ} 10^{\prime} 17.84^{\prime \prime} \mathrm{S}, 119^{\circ} 54^{\prime} 28.4^{\prime \prime} \mathrm{E}$, troglofauna (12. Nov. 2009, P. Bell, S. Callan), deposited in WAM (T108168, PBI_OON 46562).

ETYMOLOGY: This species is named for Shae Callan, collector of the type specimens.

DiAgnosis: Males of $P$. callani can be separated from those of all other blind
Prethopalpus species without a dorsal scutum by having anteriorly swollen chelicerae (figs. 513, 514), and females by having an internal genital structure not reaching the posterior spiracle groove (fig. 525).

MALE (PBI_OON 46576, figs. 508-517): Total length 1.36. Prosoma, mouthparts, and palp pale orange, carapace broadly oval in dorsal view, pars cephalica flat in lateral view, surface smooth, margin widely rebordered, front darker; Clypeus curved downward in front view, sloping forward in lateral view; Sternum longer than wide, not fused to carapace, surface smooth, distance between 


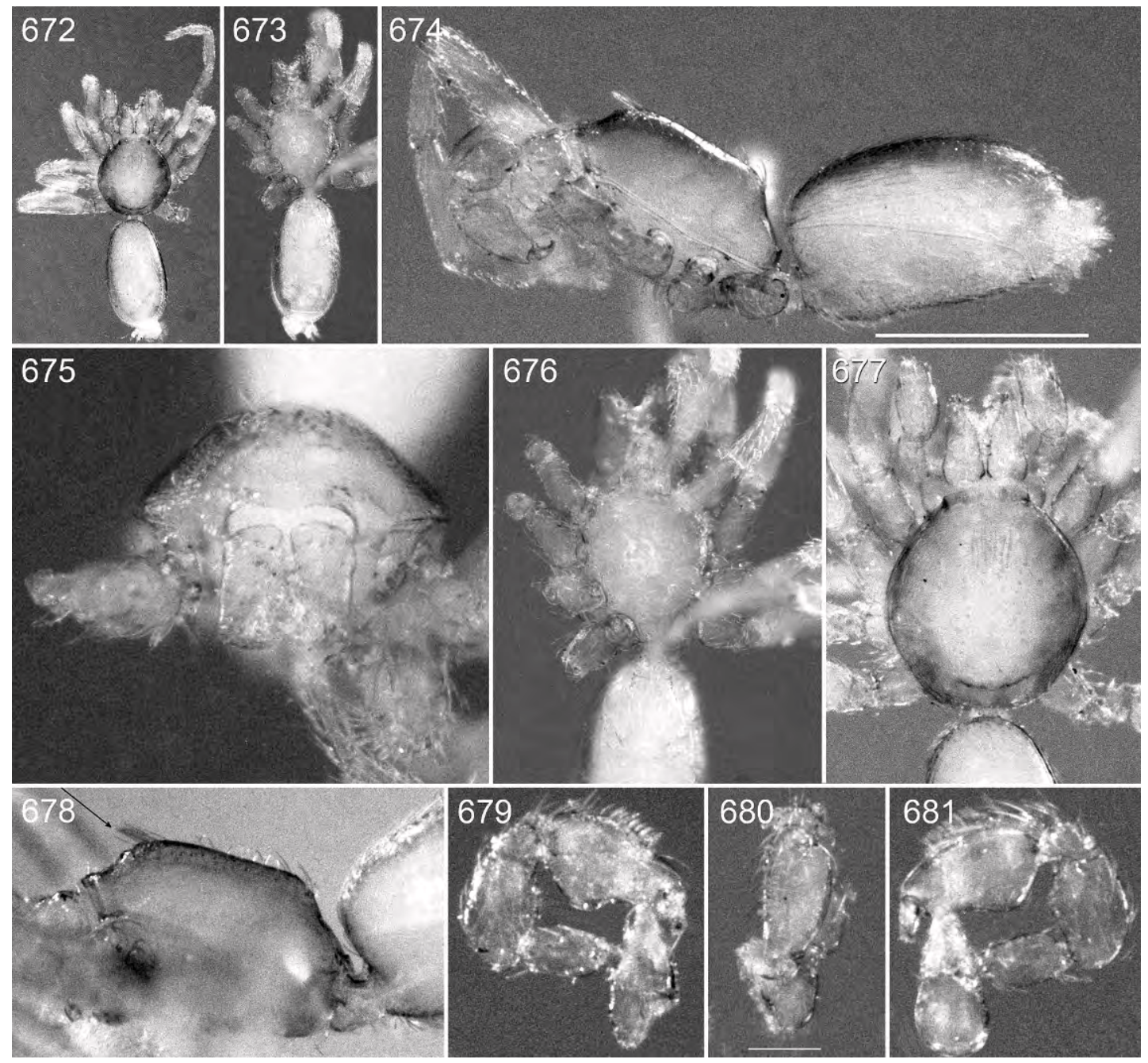

Figs. 672-681. Prethopalpus platnicki, n. sp., male (PBI_OON 21352). 672. Habitus, dorsal view. 673. Same, ventral view. 674. Same, lateral view. 675. Prosoma, anterior view. 676. Same, ventral view. 677. Same, dorsal view. 678. Same, lateral view. 679. Male palp, prolateral view. 680. Same, dorsal view. 681. Same, retrolateral view.

coxae approximately equal. Labium not fused to sternum. Endites twice as long as wide, directed medially. Abdomen ovoid; dorsum soft portions white; book lung covers large. Dorsal scutum weakly sclerotized, white, without color pattern, covering less than $1 / 2$ of abdomen, less than $1 / 4$ abdomen width, surface smooth, scutum same color as abdomen; epigastric scutum not protruding; postepigastric scutum weakly sclerotized, white, short, only around epigastric furrow, without posteriorly directed lateral apodemes; spinneret scutum absent.
Legs: white; patella plus tibia I nearly as long as carapace. Genitalia: epigastric region with sperm pore small, circular, situated at level of anterior spiracles. Palpal patella basally rounded, ventrally only slightly bulging; bulb pyriform, ventrally bulging, with long, narrow embolar tip (figs. 515-517).

Female (PBI_OON 46564, figs. 518525): Total length 1.43. Postepigastric scutum not fused to epigastric scutum. Legs: patella plus tibia I shorter than carapace. Epigastric area with large oval epigynal opening, inter- 
nal genital structure triangular, not reaching groove of posterior spiracles (fig. 525).

Other MAterial ExAmined: AUSTRALIA: Western Australia: Yeelirrie Station,

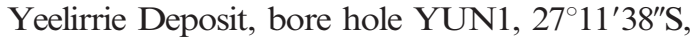
$119^{\circ} 51^{\prime} 20^{\prime \prime} \mathrm{E}$, stygofauna haul, 10 Mar. 2009, staff from Subterranean Ecology (WAM T97287, PBI_OON 46553), 1 ş; Yeelirrie, $87 \mathrm{~km} \mathrm{~S}$ of Wiluna, bore YYAC118, $27^{\circ} 10^{\prime} 28.8^{\prime \prime} \mathrm{S}, 119^{\circ} 53^{\prime} 23.06^{\prime \prime} \mathrm{E}$, stygofauna net haul, 12 Nov. 2009, P. Bell, S. Callan (WAM T108169, PBI_OON 46563), 1 s; Yeelirrie, $87 \mathrm{~km} \mathrm{~S}$ of Wiluna, bore YYHC0110, $27^{\circ} 06^{\prime} 43.96^{\prime \prime} \mathrm{S}, \quad 119^{\circ} 49^{\prime} 46.19^{\prime \prime} \mathrm{E}, \quad$ stygofauna net haul, 14 Oct. 2010, S. Callan, P. Bell (WAM T108170, PBI_OON 46578), 1 s .

DistRIBUTION: This species is known only from subterranean environments in Yeelirrie Station, situated in the Pilbara region of Western Australia (map 10).

Prethopalpus cooperi Baehr and Harvey, new species

Figures 526-538; map 10

TyPe: AUSTRALIA: Western Australia: Male holotype from Cunyu Station, State Bore Field bore 97U, $25^{\circ} 46^{\prime} 50^{\prime \prime} \mathrm{S}, 120^{\circ} 06^{\prime} 27^{\prime \prime} \mathrm{E}$ (22 July 2008, W.F. Humphreys, S. Cooper, J.M. Waldock), deposited in WAM (T103074, PBI_OON 46539).

ETYMOLOGY: This species is named for Steven Cooper of the South Australian Museum, one of the collectors of the holotype.

DiAgnosis: Males resemble those of $P$. alexanderi and $P$. oneillae in having a dorsal scutum covering the whole abdomen and no eyes, but can be separated from these species by the cylindrical abdomen (fig. 526) and the strongly bulging epigastric area (in lateral view) (fig. 538).

Male (PBI_OON 46539, figs. 526-538): Total length 1.26. Prosoma, mouthparts, palp, legs, and abdominal scuta yellow-brown, carapace ovoid in dorsal view, pars cephalica flat in lateral view, surface of elevated portion of pars cephalica smooth, sides granulate, only center of carapace smooth. Clypeus straight in front view, sloping forward in lateral view. Sternum fused to carapace, surface finely reticulate, microsculpture covering entire surface. Endites twice as long as wide, directed medially. Abdomen cylindrical; book lung covers large; pedicel ribbed, scutum not extending far dorsal of pedicel. Dorsal scutum weakly sclerotized, pale orange, covering full length of abdomen, no soft tissue visible from above, surface smooth; epigastric scutum surrounding pedicel, strongly protruding, with two darker lateral patches at height of anterior spiracles; postepigastric scutum weakly sclerotized, long, almost rectangular, covering about $3 / 4$ of abdominal length, fused to epigastric scutum, without posteriorly directed lateral apodemes. Legs: patella plus tibia I shorter than carapace. Genitalia: epigastric region with sperm pore small, circular, situated in front of anterior spiracles. Palpal patella about as long as femur, slightly larger than femur, patella not bulging ventrally, with rounded base; bulb pear shaped, extremely bulging ventrally, with narrow curled embolar tip (figs. 534-536).

FeMAle: Unknown.

Other MAterial ExAmined: None.

DISTRIBUTION: This species is known only from a subterranean calcrete deposit on Cunyu Station, situated in the Yilgarn region of Western Australia (map 10).

\section{Prethopalpus eberhardi Baehr and Harvey, new species Figures 539-555; map 9}

TyPeS: AUSTRALIA: Western Australia: Male holotype from ca. $13 \mathrm{~km}$ NE of Yarrie Homestead, borehole \#CA0011R, stygofauna haul, $20^{\circ} 38^{\prime} 57.1^{\prime \prime} \mathrm{S}, 120^{\circ} 18^{\prime} 27.2^{\prime \prime} \mathrm{E}$ (26 Apr. 2008, S. Eberhard), deposited in WAM (T91749, PBI_OON 18855). Paratype: 1 female, Yarrie area, hole \#CA0012R, 20³9'06.1"S, 120 18'23.1" E (26 Apr. 2008, S. Eberhard), deposited in WAM (T91743, PBI_OON 18854).

ETYMOLOGY: This species is named for Stefan Eberhard, collector of the type specimens and many other fascinating subterranean organisms.

DiAgNOSIS: Males and females resemble those of $P$. infernalis, $P$. alexanderi, $P$. oneillae, and $P$. cooperi in having an abdominal dorsal scutum and no eyes, but can be separated from these species by the postepigastric scutum covering $2 / 3$ of the abdomen (figs. 540, 546). Males can be separated from $P$. infernalis by the palpal patella with a strong ventral swelling (figs. 543, 545). 
MAle (PBI_OON 18855, figs. 539-545): Total length 1.07. Prosoma, mouthparts, palp, legs, and abdominal scuta pale orange, carapace, ovoid in dorsal view, pars cephalica slightly elevated in lateral view, surface of elevated portion of pars cephalica smooth, sides granulate. Clypeus curved downward in front view, sloping forward in lateral view. Eyes absent. Sternum fused to carapace, surface smooth. Labium not fused to sternum. Endites twice as long as wide, with tiny tooth at anteromedian tip. Abdomen ovoid; book lung covers large; pedicel ribbed. Dorsal scutum weakly sclerotized, covering more than $3 / 4$ of abdomen, no soft tissue visible from above, surface smooth; epigastric scutum not protruding; postepigastric scutum weakly sclerotized, long, semicircular, covering about $3 / 4$ of abdominal length, fused to epigastric scutum, without posteriorly directed lateral apodemes; spinneret scutum with fringe of stout setae. Legs yellow; patella plus tibia I nearly as long as carapace. Epigastric region with sperm pore small, oval, situated at level of anterior spiracles. Palpal patella about as long as femur, patella ventrally swollen, with rounded base; bulb pear shaped bulging ventrally, with narrow curled embolar tip swelling (figs. 543-545).

Female (PBI_OON 18854, figs. 546555): Total length 1.22. Epigastric area with broad slitlike epigynal opening, internal genital structure with free, inverted teardrop-shaped anterior sclerite, and a long sinuous posterior tube, posterior part not reaching groove of posterior spiracles (fig. 555). Dorsal scutum covering ca. $3 / 4$ of abdomen; postepigastric scutum almost semicircular, covering about $2 / 3$ of abdominal length, not fused to epigastric scutum.

Other MAterial Examined: None.

DISTRIBUTION: This species is known only from a single location in the Pilbara region of Western Australia (map 9).

Prethopalpus framenaui Baehr and Harvey, new species

Figures 556-575; map 9

Types: AUSTRALIA: Western Australia: Male holotype from Nambi Station, Shady Well calcrete, bore litter trap 11, bore, $28^{\circ} 23^{\prime} 54^{\prime \prime} \mathrm{S}, 122^{\circ} 11^{\prime} 55^{\prime \prime} \mathrm{E}$ (July 2010, W.F.
Humphreys, S. Cooper), deposited in WAM (T104043, PBI_OON 46542). Paratypes: 1 female, collected with holotype (WAM T111751, PBI_OON 23554); 1 male, collected with holotype (WAM T104045, PBI_OON 46544).

ETYMOLOGY: This species is named for Volker Framenau for his contributions to arachnological research.

Diagnosis: Males and females of $P$. framenaui resemble those of $P$. boltoni and $P$. humphreysi in lacking a dorsal abdominal scutum and eyes, and $P$. humphreysi in sharing a well-sclerotized carapace but lacking the dark book lung covers. Males can be easily separated from $P$. humphreysi by having a much shorter, more pear-shaped palpal bulb (figs. 564, 566), and females can be recognized by the long, straight posterior tube in the genitalia, with only the posterior part sinuous, reaching the posterior spiracular groove (fig. 575).

MALE (PBI_OON 46542, figs. 556-566): Total length 1.22. Prosoma, mouthparts, palp, legs, and abdominal scuta pale orange, carapace ovoid in dorsal view, pars cephalica flat in lateral view, surface of elevated portion of pars cephalica smooth, sides finely reticulate. Clypeus curved downward in front view, sloping forward in lateral view. Sternum fused to carapace, surface smooth. Endites twice as long as wide, directed medially. Abdomen ovoid; book lung covers small; pedicel unmodified. Dorsal scutum absent; epigastric scutum surrounding pedicel, not protruding; postepigastric scutum weakly sclerotized, almost semicircular, only around epigastric furrow, fused to epigastric scutum, anterior margin unmodified, without posteriorly directed lateral apodemes; spinneret scutum with fringe of stout setae. Legs: patella plus tibia I shorter than carapace. Genitalia: epigastric region with sperm pore small, circular, situated in front of anterior spiracles. Palp patella not bulging, ventrally rounded at base, bulb strongly protruding ventrally with a short coiled embolar tip (figs. 563-566).

Female (PBI_OON 23554, figs. 567575): Total length 1.35. Abdomen with postepigastric scutum not fused to epigastric scutum. Epigastric area with small round epigynal opening, internal genital structure 


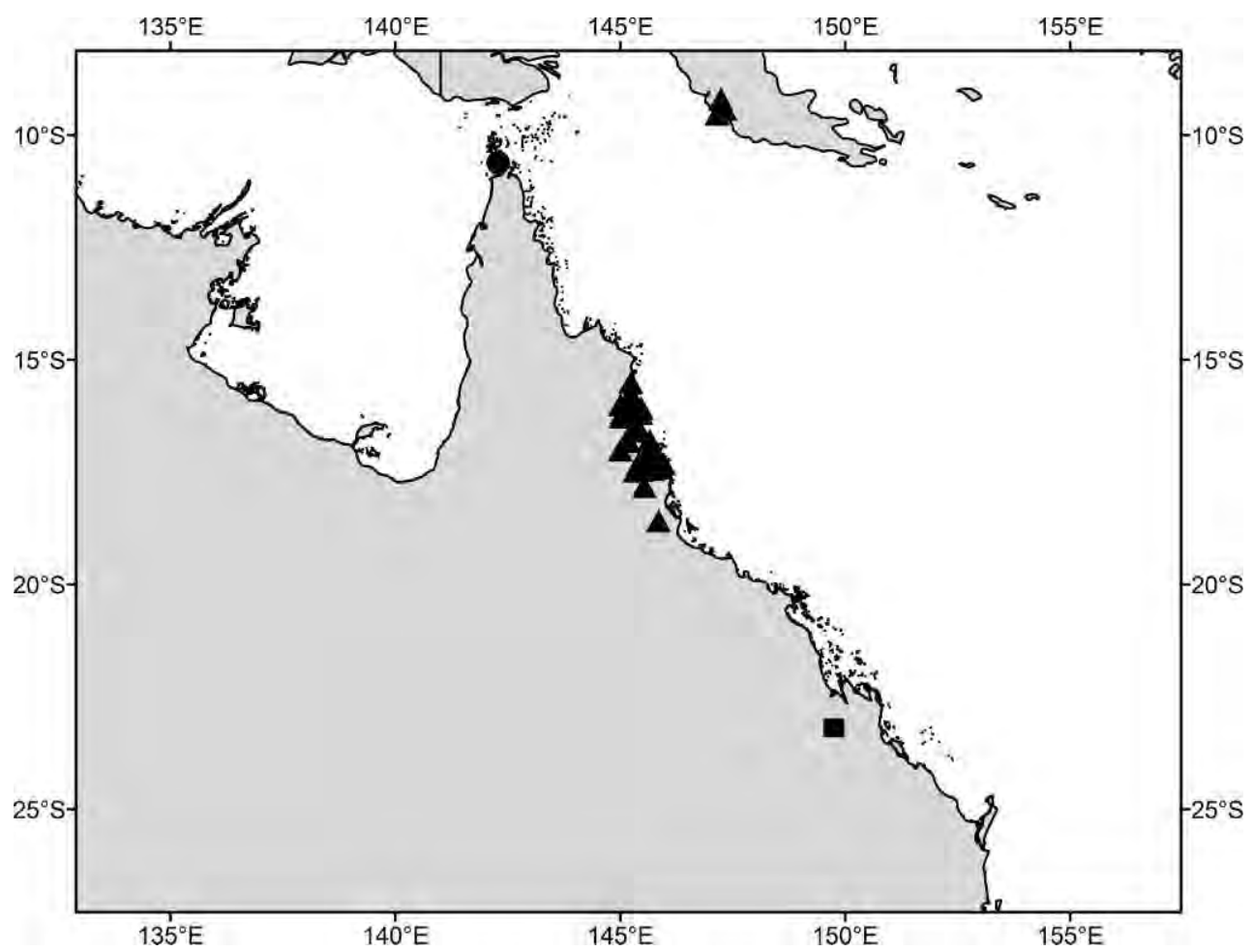

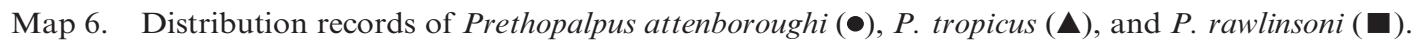

with free, needlelike anterior sclerite, and a long sinuous posterior tube, posterior part reaching groove of posterior spiracles (fig. 575).

Other Material Examined: None.

DISTRIBUTION: This species is known only from a subterranean calcrete from Nambi Station, situated in the Yilgarn region of Western Australia (map 7).

\section{Prethopalpus humphreysi Baehr and} Harvey, new species

Figures 576-599; map 9

Types: AUSTRALIA: Western Australia: Male holotype from Sturt Meadows Station, bore $128,28^{\circ} 42^{\prime} 04^{\prime \prime} \mathrm{S}, 120^{\circ} 53^{\prime} 55^{\prime \prime} \mathrm{E}$, litter traps in bore (24 July 2008, W.F. Humphreys, S. Cooper, J.M. Waldock), deposited in WAM (T103073, PBI_OON 46538). Paratypes: 1 female collected with holotype, deposited in WAM (T111752, PBI_OON 23555); 1 female collected with holotype, deposited in WAM (T104044, PBI_OON 46543); 1 female, from Sturt Meadows Station, bore $128,28^{\circ} 42^{\prime} 30^{\prime \prime} \mathrm{S}, 120^{\circ} 53^{\prime} 39^{\prime \prime} \mathrm{E}$
(Nov. 2006, A. Allford), deposited in WAM (T103076, PBI_OON 46541).

ETYMOLOGY: This species is named for Bill Humphreys of the Western Australian Museum, one of the collectors of the types.

Diagnosis: Males and females of this species resemble those of $P$. boltoni and $P$. framenaui in lacking an abdominal dorsal scutum and eyes; they differ from P. framenaui in sharing a well-sclerotized carapace but having dark book lung covers (fig. 578). Males can be easily separated from $P$. framenaui by the much longer bulb, which is ventrally more pointed (in lateral view) (figs. 587, 588); females can be recognized by the shorter straight posterior tube in the genitalia, and the larger sinuous part, not reaching the posterior spiracular groove (fig. 599).

MAle (PBI_OON 46538, figs. 576-589): Total length 1.28. Prosoma, mouthparts, and palp pale orange, carapace ovoid in dorsal view, pars cephalica flat in lateral view, surface of elevated portion of pars cephalica smooth, sides finely reticulate. Clypeus 


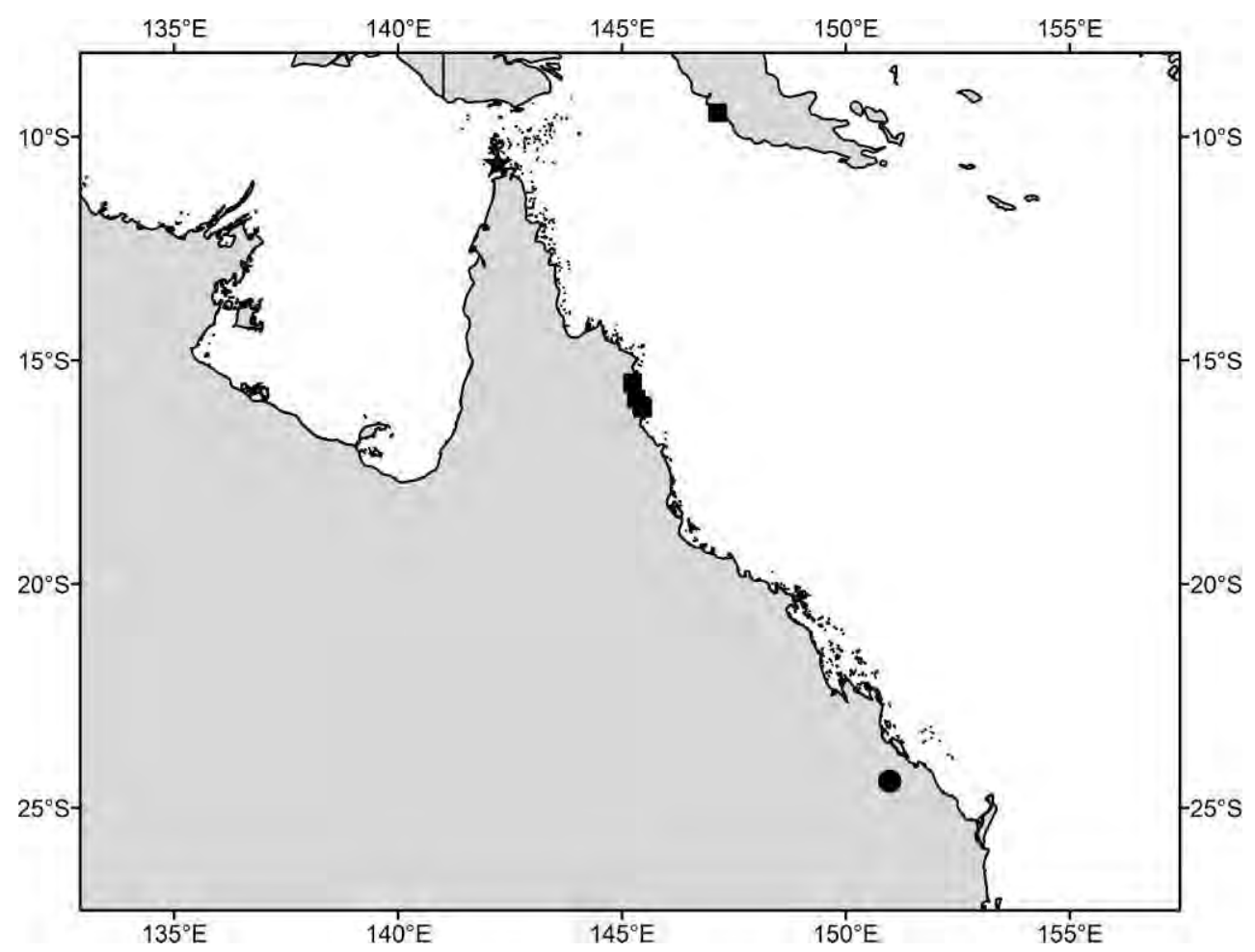

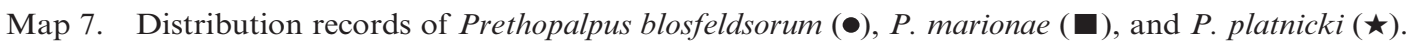

curved downward in front view, sloping forward in lateral view. Sternum fused to carapace, surface smooth. Endites twice as long as wide. Abdomen ovoid; book lung covers large, brown, darker than abdomen. Dorsal scutum absent; epigastric scutum surrounding pedicel, slightly protruding; postepigastric scutum absent, postepigastric area slightly protruding; spinneret scutum with fringe of stout setae. Legs white; patella plus tibia I nearly as long as carapace. Genitalia: epigastric region with sperm pore large, triangular with rounded angles, situated in front of anterior spiracles. Palpal patella longer than femur, ventrally slightly bulging, posteriorly rounded, connection to femur subbasally; bulb ventrally strongly bulging and with elongated distal embolar tip (figs. 584-588).

Female (PBI_OON 23555, figs 590599): Total length 1.58. Epigastric area with slitlike epigynal opening, internal genital structure with free, needlelike, T-shaped anterior sclerite that is medially thickened, and a long sinuous posterior tube, posterior part not reaching groove of posterior spiracles (fig. 599).

Other Material Examined: None.

Distribution: This species is known only from subterranean calcrete deposits at Sturt Meadows Station, in the Yilgarn region of Western Australia (map 9).

Prethopalpus infernalis (Harvey and Edward), new combination Figures 600-607; map 6

Camptoscaphiella infernalis Harvey and Edward, 2007b: 14-16, figs. 9-13.

TyPe: AUSTRALIA: Western Australia: Male holotype from Cape Range region, Learmonth Limestone bore \#LL11, $22^{\circ} 13^{\prime} 27^{\prime \prime} \mathrm{S}, 1^{\circ} 04^{\circ} 52^{\prime \prime} \mathrm{E}$, depth $31 \mathrm{~m}$ (6 Apr. 2001, R.D. Brooks), deposited in WAM (T54533, PBI_OON 46550).

Diagnosis: Males resemble those of $P$. eberhardi, $P$. alexanderi, $P$. oneillae, and $P$. cooperi in having an abdominal dorsal scutum and no eyes, but can be separated from these species by the postepigastric 


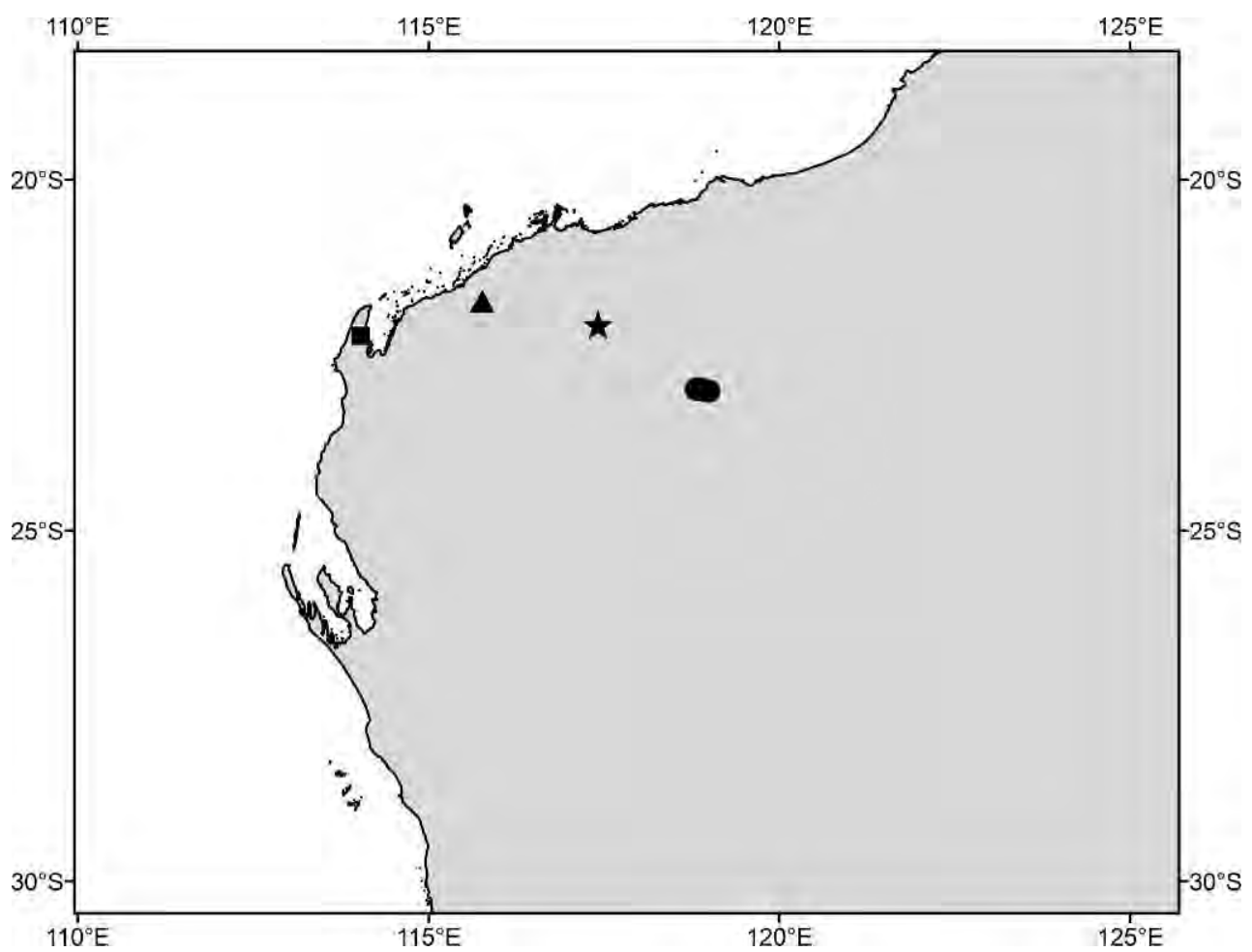

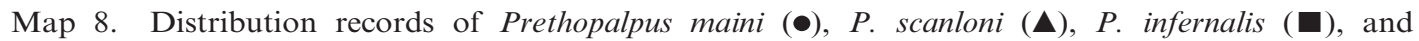
P. oneillae ( $\star$ ).

scutum covering only $1 / 2$ of the abdomen (fig. 601).

MALE (PBI_OON 46550, figs.600-607): Total length 1.19. Prosoma, mouthparts, palp, and abdominal scuta pale orange, carapace ovoid in dorsal view, pars cephalica flat in lateral view, surface smooth. Clypeus curved downward in front view, vertical in lateral view. Sternum fused to carapace, surface smooth. Anteromedian tip of endites unmodified. Abdomen ovoid; book lung covers large. Dorsal scutum weakly sclerotized, covering $70 \%$ of abdomen, ca. $1 / 2$ of abdomen width, surface smooth; epigastric area not protruding; postepigastric scutum weakly sclerotized, almost semicircular, covering about $1 / 2$ of abdominal length, fused to epigastric scutum, without posteriorly directed lateral apodemes; spinneret scutum with fringe of stout setae. Legs: yellow; patella plus tibia I nearly as long as carapace. Genitalia: epigastric region with sperm pore small, circular, situated in front of anterior spiracles. Palp patella ventrally slightly bulging, basally rounded, bulb perform, slightly bulging, ventrally with short narrow embolar tip (figs. 603, 604).

FEMAle: Unknown.

Other Material Examined: None.

DISTRIBUTION: This species is known only from a borehole at Cape Range in northwestern Australia (map 6).

\section{Prethopalpus julianneae Baehr and} Harvey, new species

Figures 608-617; map 10

TyPes: AUSTRALIA: Western Australia: Male holotype from mining Area C, bore SF0569R, c. $100 \mathrm{~km}$ NW of Newman, troglofauna, $-39 \mathrm{~m}$ (18 Mar. 2010, J.S. Cocking, D.C. Main), deposited in WAM (T108273, PBI_OON 46587).

ETYMOLOGY: This species is named for Julianne Waldock in recognition of her arachnological prowess. 


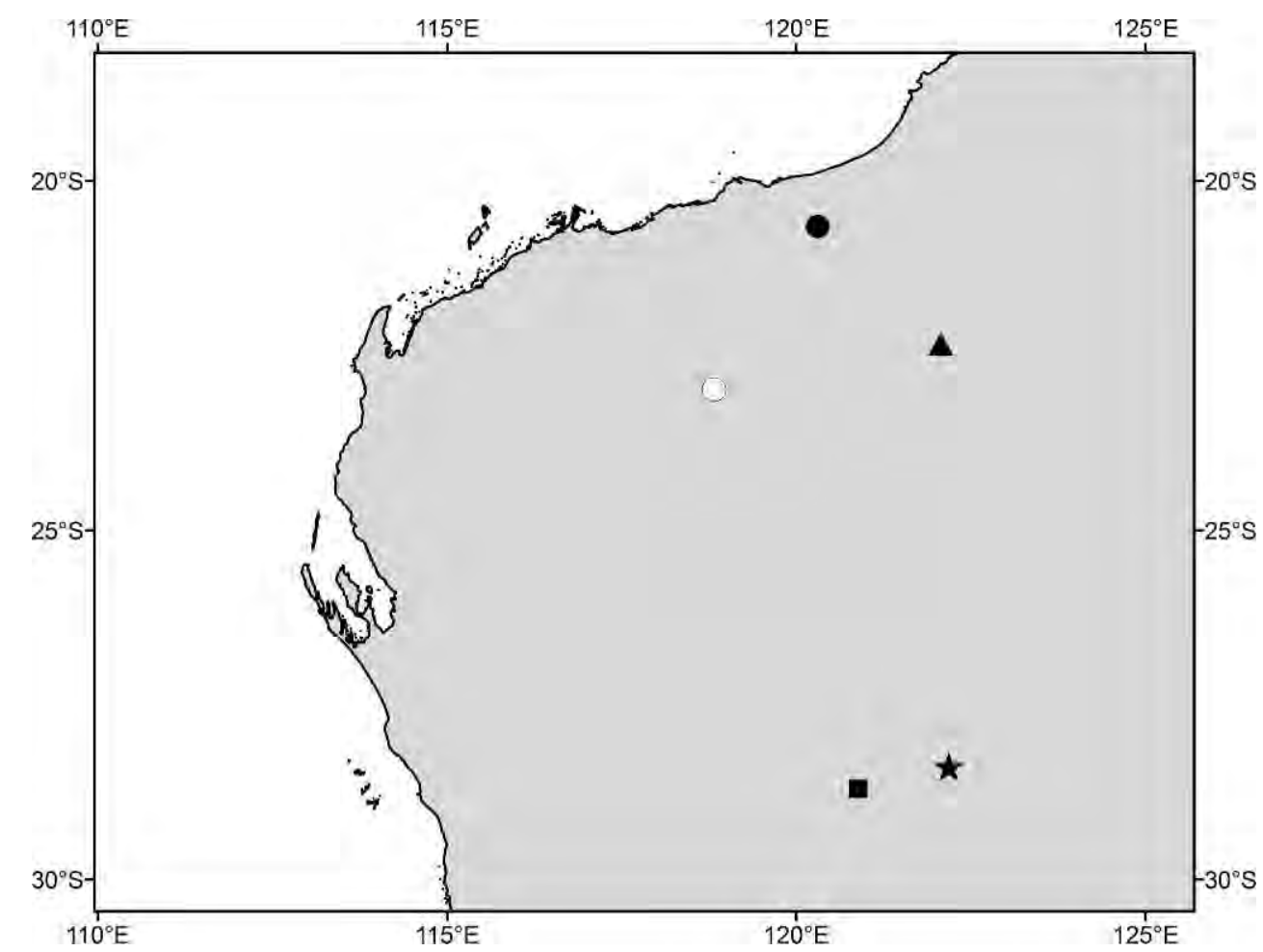

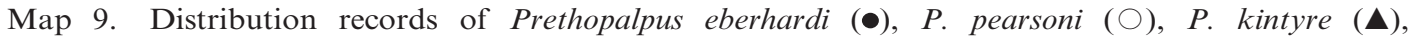
$P$. humphreysi $(\boldsymbol{\square})$, and $P$. framenaui $(\star)$.

DiAGNOSIS: Males can be separated from those of all other blind Prethopalpus species with a dorsal scutum by having endites about 1.5 times as long as wide (fig. 612).

MALE (PBI_OON 46587, figs. 608-617): Total length 1.26. Prosoma, mouthparts, legs, palp, and abdominal scuta pale orange, carapace broadly oval in dorsal view, pars cephalica flat in lateral view, surface smooth. Clypeus straight in front view, sloping forward in lateral view. Sternum longer as wide, not fused to carapace, surface smooth, distance between coxae approximately equal. Endites about 1.5 times as long as wide. Abdomen ovoid; book lung covers large. Dorsal scutum weakly sclerotized, covering full length of abdomen, more than $1 / 2$ to most of abdomen width, surface smooth; epigastric scutum not protruding; postepigastric scutum weakly sclerotized, long, semicircular, covering about $3 / 4$ of abdominal length, fused to epigastric scutum, without posteriorly directed lateral apodemes; spinneret scutum absent; spinneret scutum without fringe of setae. Legs: patella plus tibia I shorter than carapace. Genitalia: epigastric region with sperm pore small, circular, situated at level of anterior spiracles. Palpal patella ventrally slightly bulging, basally rounded, bulb pyriform, ventrally rounded with wide tip (figs. 615-617).

FEMALE: Unknown.

Other Material Examined: None.

Distribution: This species is known only from subterranean habitats in the Pilbara region of Western Australia (map 10).

Prethopalpus kintyre Baehr and Harvey, new species

Figures 618-625; map 9

TYPE: AUSTRALIA: Western Australia: Female holotype, Kintyre, ca. $60 \mathrm{~km} \mathrm{~S}$ of Telfer, $22^{\circ} 20^{\prime} 07.5^{\prime \prime} \mathrm{S}, 122^{\circ} 04^{\prime} 51.5^{\prime \prime} \mathrm{E}$, troglofauna, $-23 \mathrm{~m}$, (28 July 2010, D.C. Main, G.B. Pearson, deposited in WAM (T108263, PBI_OON 46574). 


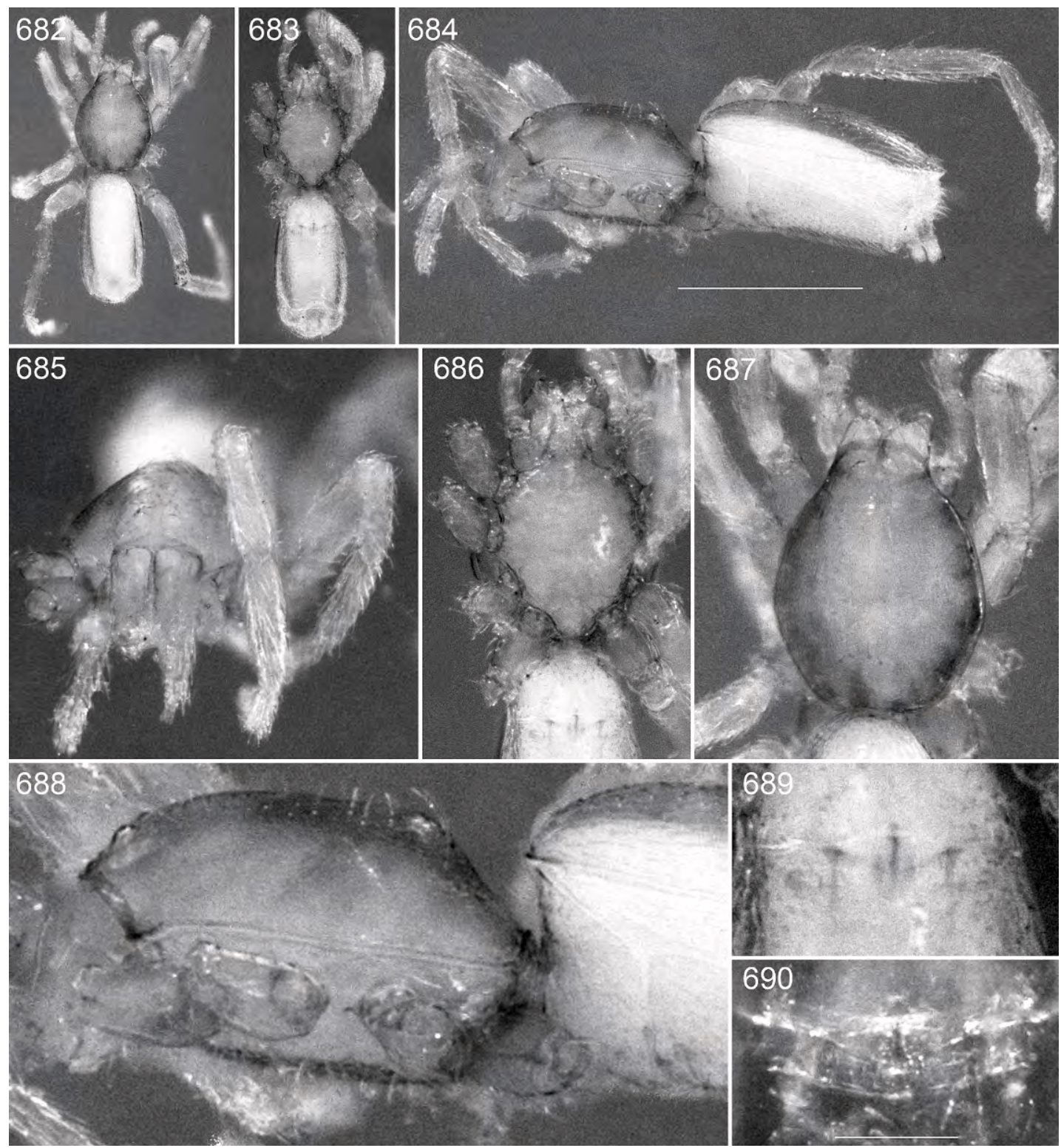

Figs. 682-690. Prethopalpus platnicki, n. sp., female (PBI_OON 23402). 682. Habitus, dorsal view. 683. Same, ventral view. 684. Same, lateral view. 685. Prosoma, anterior view. 686. Same, ventral view. 687. Same, dorsal view. 688. Same, lateral view. 689. Female epigyne, ventral view. 690. Female epigyne, dorsal view.

ETYMOLOGY: The species name is a noun in apposition derived from the type locality.

DiAgNosis: Females can be separated from those of all other blind Prethopalpus species without a dorsal scutum by a greater distance between coxae II-III than between coxae I-II and II-IV (fig. 622).
Female (PBI_OON 46574, figs. 618625): Total length 1.09. Prosoma, legs, palp, and abdominal scuta white, mouthparts pale orange, carapace elongated hexagonal in dorsal view, pars cephalica flat in lateral view, surface smooth. Clypeus curved downward in front view, sloping forward in lateral 


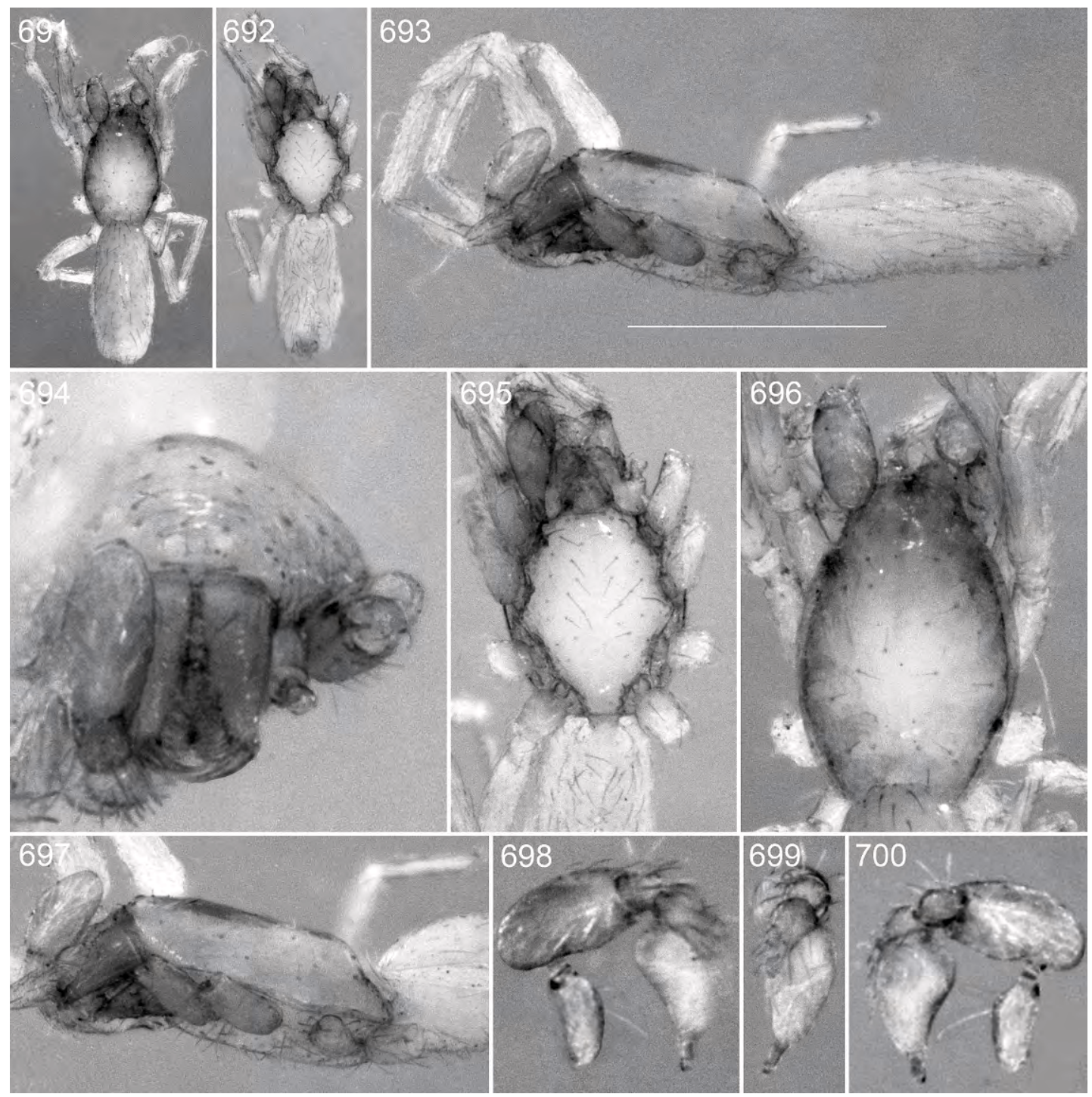

Figs. 691-700. Prethopalpus pearsoni, n. sp., male (PBI_OON 46591). 691. Habitus, dorsal view. 692. Same, ventral view. 693. Same, lateral view. 694. Prosoma, anterior view. 695. Same, ventral view. 696. Same, dorsal view. 697. Same, lateral view. 698. Male palp, prolateral view. 699. Same, dorsal view. 700. Palp, retrolateral view.

view. Sternum not fused to carapace, surface smooth, distance between coxae II and III greater than distance between coxae I and II, and coxae III and IV. Labium not fused to sternum, anterior margin not indented at middle. Endites twice as long as wide directed medially. Abdomen: book lung covers large; pedicel unmodified. Dorsal, epigastric, postepigastric, and spinneret scuta absent. Legs: patella plus tibia I shorter than carapace. Epigastric area with small epigynal opening, internal genital structure with free, needlelike anterior sclerite, and a long sinuous posterior tube, posterior part reaching groove of posterior spiracles (fig. 625).

Other Material Examined: None.

Distribution: This species is known only from subterranean environments in the Pilbara region of Western Australia (map 9). 
Figs. 701-708. Prethopalpus rawlinsoni, n. sp., male (PBI_OON 07129). 701. Habitus, dorsal view. 702. Same, ventral view. 703. Prosoma, ventral view. 704. Habitus, lateral view. 705. Male palp, prolateral view. 706. Same, dorsal view. 707. Patella, dorsal view. 708. Palp, retrolateral view.

Prethopalpus maini Baehr and Harvey, new species

Figures 626-643; map 8

TyPes: AUSTRALIA: Western Australia: Holotype male from mining Area C, bore SF0288R, c. $100 \mathrm{~km} \mathrm{NW}$ of Newman, $22^{\circ} 59^{\prime} 05.8^{\prime \prime} \mathrm{S}, \quad 118^{\circ} 49^{\prime} 06.4^{\prime \prime} \mathrm{E}, \quad$ troglofauna, $-27 \mathrm{~m}$, (18 Mar. 2010, J.S. Cocking, D.C. Main), deposited in WAM (T108272, PBI_OON 46586). Paratype male from same location (27 June 2010, G. B. Pearson, M. K. Curran), deposited in WAM (T108276, PBI_OON 46590).

OTHer Material Examined: AUSTRALIA: Western Australia: mining Area C, bore SF0140R, c. $100 \mathrm{~km} \mathrm{NW}$ of Newman, $23^{\circ} 00^{\prime} 35.2^{\prime \prime} \mathrm{S}, \quad 118^{\circ} 58^{\prime} 57.4^{\prime \prime} \mathrm{E}, \quad$ troglofauna, $-56 \mathrm{~m}, 16$ Feb. 2010, G.B. Pearson, D. C. Main, 1 \& (WAM T108269, PBI_OON 46583); mining Area C, bore SF0140R, c. $100 \mathrm{~km} \mathrm{NW}$ of Newman, $23^{\circ} 00^{\prime} 49.2^{\prime \prime} \mathrm{S}$, $118^{\circ} 59^{\prime} 18.7^{\prime \prime} \mathrm{E}$, troglofauna, -93m, $17 \mathrm{Feb}$.
2010, G. B. Pearson, M. K. Curran, 1 के (WAM T108270, PBI_OON 46584); mining Area C, c. $100 \mathrm{~km} \mathrm{NW}$ of Newman, bore SF0383R, $22^{\circ} 59^{\prime} 05.5^{\prime \prime} \mathrm{S}, 118^{\circ} 51^{\prime} 13.0^{\prime \prime} \mathrm{E}$, troglofauna, $-57 \mathrm{~m}, 19$ Mar. 2010, J.S. Cocking, D.C. Main, 1 ㅇ (WAM T108274, PBI_OON 46588).

ETYMOLOGY: This species is named for Dean Main, collector of the holotype.

DiAgNosis: Males and females resemble those of $P$. eberhardi in having a dorsal scutum covering about $80 \%$ of the abdomen but can be separated by the posteriorly rounded patella (in lateral view) in males (figs. 633, 635), and the more elongated carapace in females (fig. 641).

MALE (PBI_OON 46586, figs. 626-635): Total length 1.28. Prosoma, mouthparts, and palp pale orange, carapace oval in dorsal view, pars cephalica flat in lateral view, surface smooth. Clypeus straight in front view, sloping forward in lateral view. Sternum longer than wide, not fused to carapace, surface smooth, distance between coxae 


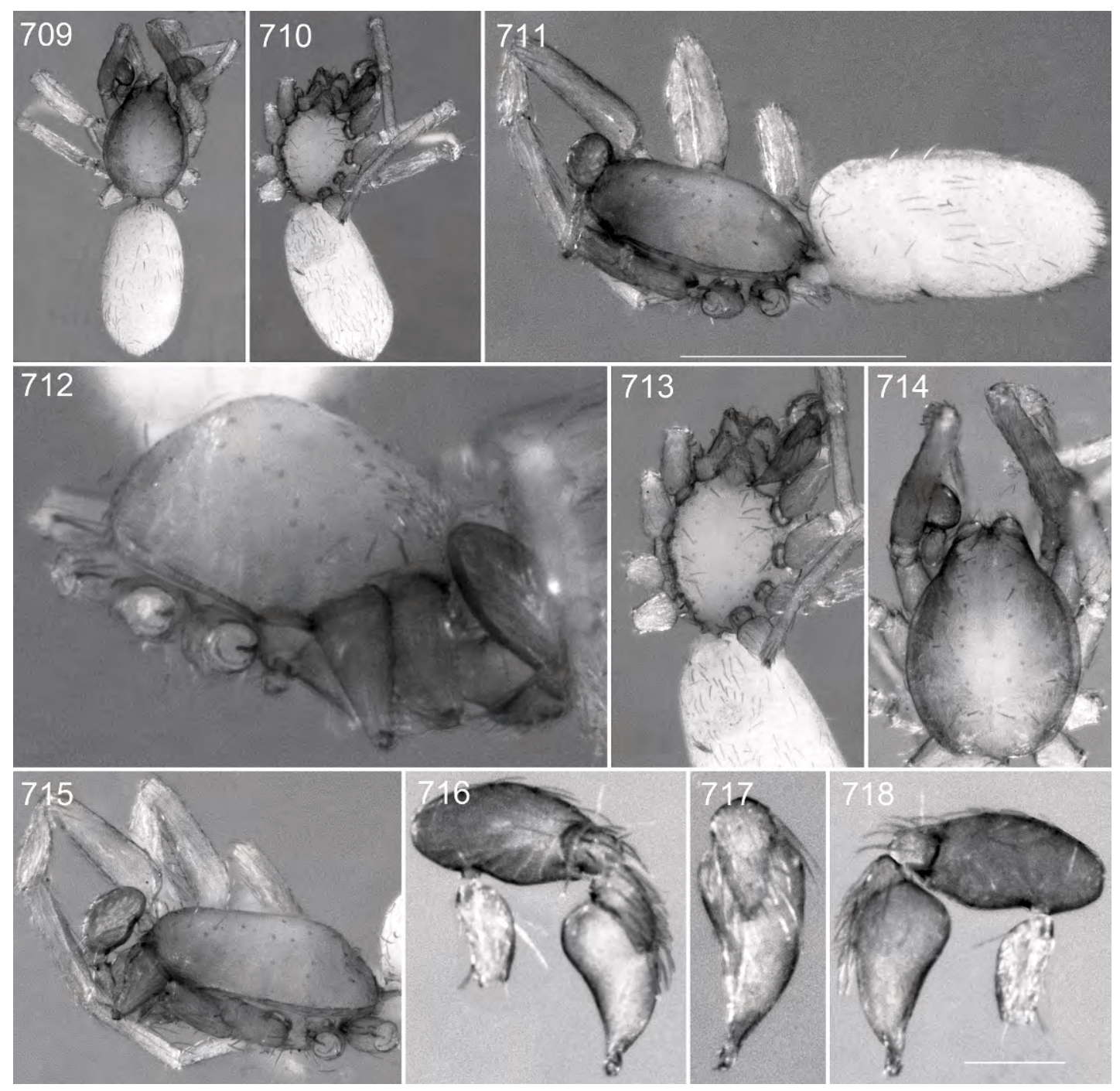

Figs. 709-718. Prethopalpus scanloni, n. sp., male (PBI_OON 07129). 709. Habitus, dorsal view. 710. Same, ventral view. 711. Same, lateral view. 712. Prosoma, anterior view. 713. Same, ventral view. 714. Same, dorsal view. 715. Same, lateral view. 716. Male palp, prolateral view. 717. Same, dorsal view. 718. Palp, retrolateral view.

approximately equal. Endites twice as long as wide, directed medially. Abdomen ovoid; book lung covers large. Dorsal scutum weakly sclerotized, white, covering $3 / 4$ of abdomen, more than $1 / 2$ of abdomen width, surface smooth, dorsal scutum very weak barely visible; epigastric scutum surrounding pedicel, not protruding; postepigastric scutum weakly sclerotized, white, long, semicircular, covering about $2 / 3$ of abdominal length, fused to epigastric scutum, without posteriorly directed lateral apodemes, postepigastric scutum very weak, nearly the same color as soft parts of the abdomen; spinneret scutum absent. Legs: white; patella plus tibia I longer than carapace. Tarsi I to IV without inferior claw. Genitalia: epigastric region with sperm pore small, circular, situated at level of anterior spiracles. Palpal patella basally rounded and ventrally bulging; bulb pyriform 


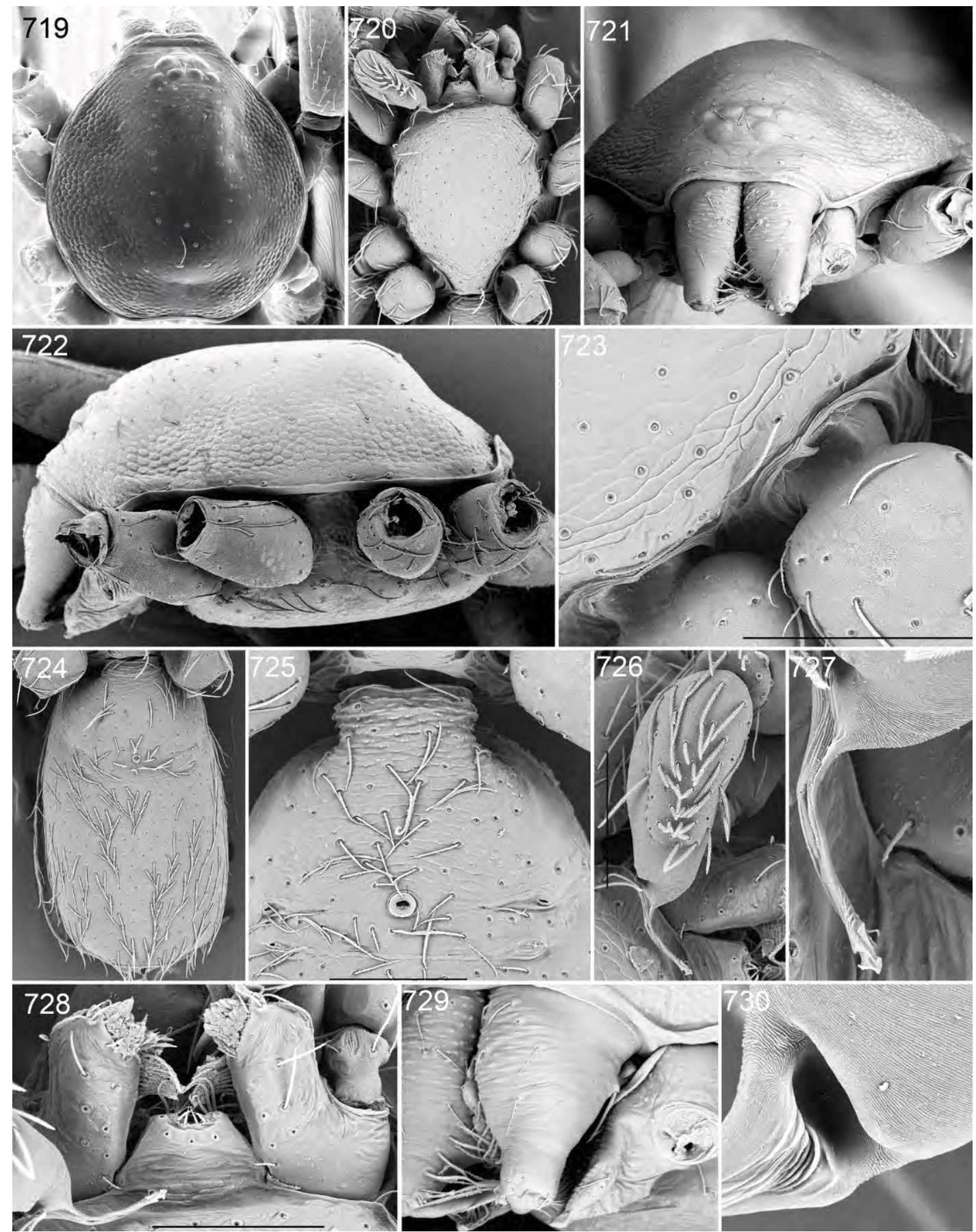

Figs. 719-730. Prethopalpus tropicus, n. sp., male, scanning electron micrographs (PBI_OON 06426). 719. Prosoma, dorsal view. 720. Same, ventral view. 721. Same, anterior view. 722. Same, lateral view. 723. Sternum, lateral margin. 724. Opisthosoma, ventral view. 725. Pedicel and sperm pore, ventral view. 726. Male palp, dorsal view. 727. Embolus, dorsal view. 728. Labium, endite, ventral view. 729. Serrula, lateral view. 730. Base of embolus, retrolateral view. 


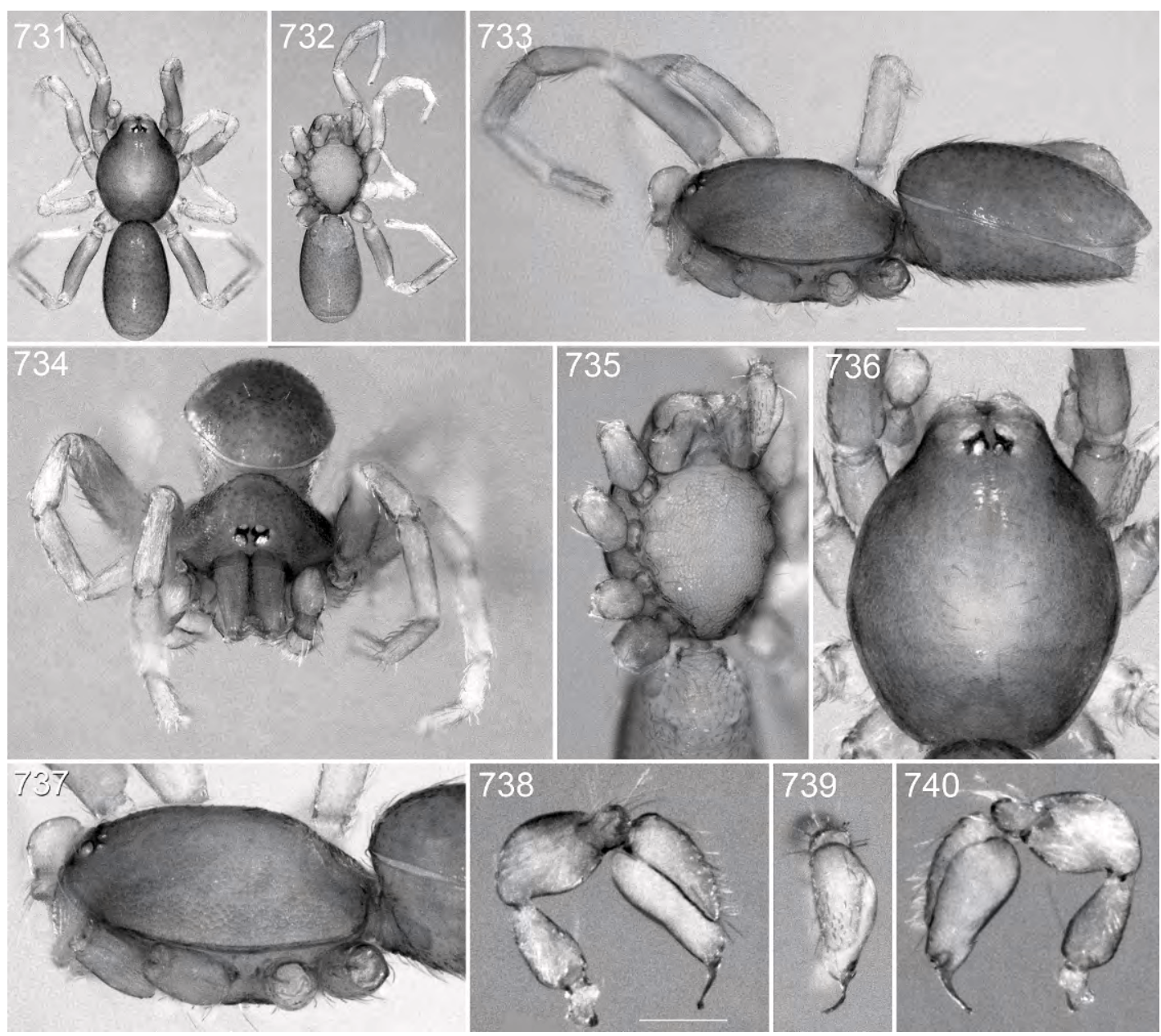

Figs. 731-740. Prethopalpus tropicus, n. sp., male (PBI_OON 23339). 731. Habitus, dorsal view. 732. Same, ventral view. 733. Same, lateral view. 734. Prosoma, anterior view. 735. Same, ventral view. 736. Same, dorsal view. 737. Same, lateral view. 738. Male palp, prolateral view. 739. Same, dorsal view. 740. Same, retrolateral view.

ventrally slightly bulging with narrowed excavated embolar tip (figs. 633-635).

FeMAle (PBI_OON 46590, figs. 636643): Total length 1.16. Epigastric area with small round epigynal opening, internal genital structure with free, needlelike globular anterior sclerite, posterior tube short not reaching groove of posterior spiracles (fig. 643).

Other Material ExAmined: None.

DistRIBUTION: This species is known only from subterranean environments northwest of Newman, in the Pilbara region of Western Australia (map 8).

\section{Prethopalpus marionae Baehr and}

Harvey, new species

Figures 128-130, 157, 158, 644-659; map 7

TYPES: AUSTRALIA: Queensland: Male holotype from $3 \mathrm{~km} \mathrm{NNE}$ of Mt Webb, $15.5000^{\circ} \mathrm{S}, 145.1500^{\circ} \mathrm{E}(30$ Apr.-3 May 1981 , A. Calder, J. Feehan), deposited in ANIC (PBI_OON 00023006). Female allotype collected with holotype, deposited in ANIC (PBI_OON 00023403).

ETYMOLOGY: The specific name is in honor of Marion Morgan, M.S.H.'s mother. 


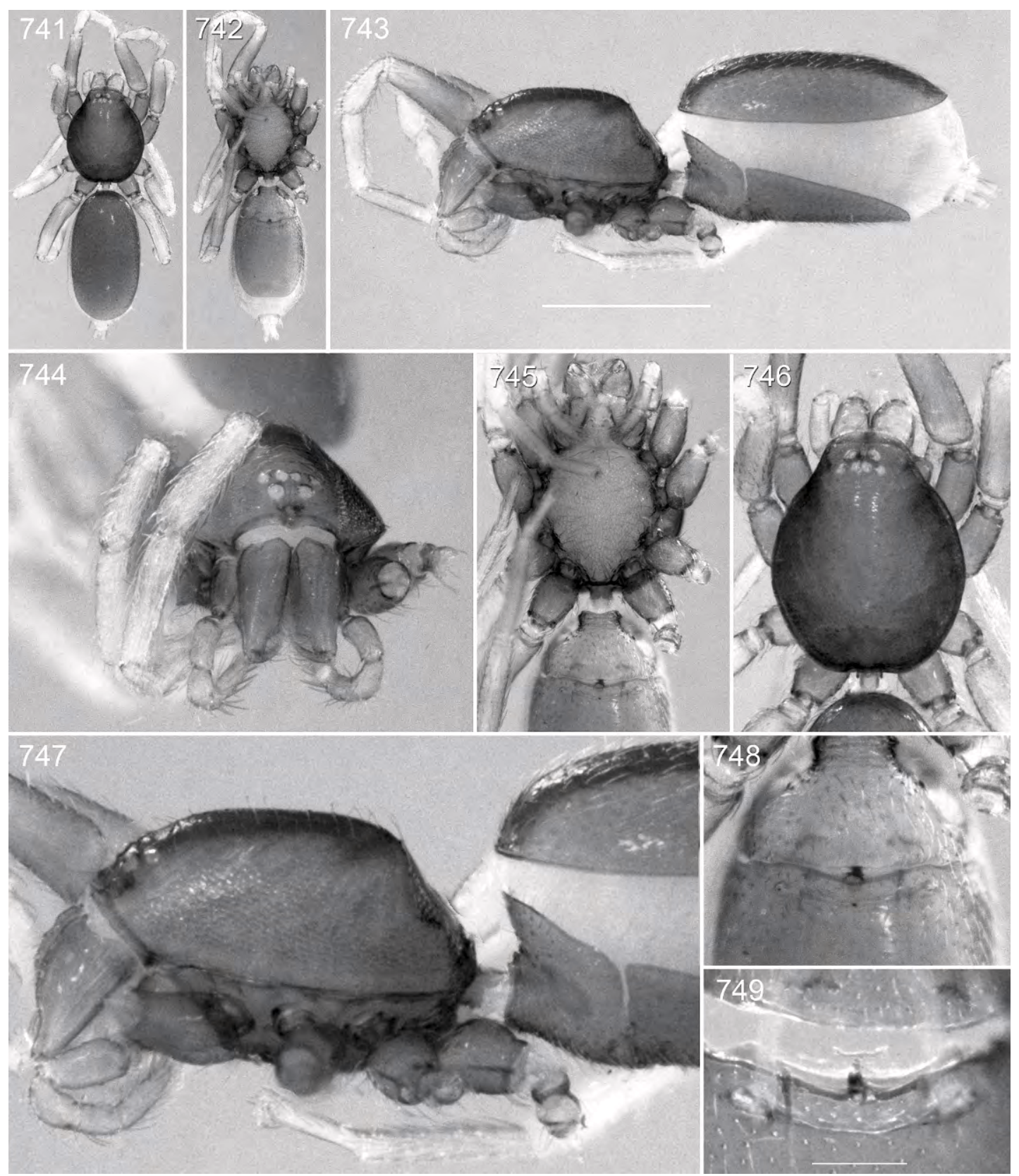

Figs. 741-749. Prethopalpus tropicus, n. sp., female (PBI_OON 23373). 741. Habitus, dorsal view. 742. Same, ventral view. 743. Same, lateral view. 744. Prosoma, anterior view. 745. Same, ventral view. 746. Same, dorsal view. 747. Same, lateral view. 748. Female epigyne, ventral view. 749. Female epigyne, dorsal view.

DiAgnosis: Males and females resemble those of $P$. tropicus in general body shape and eye sizes, but can be separated by a smooth sternal surface (fig. 648).
MALE (PBI_OON 23006, figs. 644-652): Total length 1.09. Prosoma, mouthparts, palp, and abdominal scuta yellow, surface of elevated portion of pars cephalica slightly 


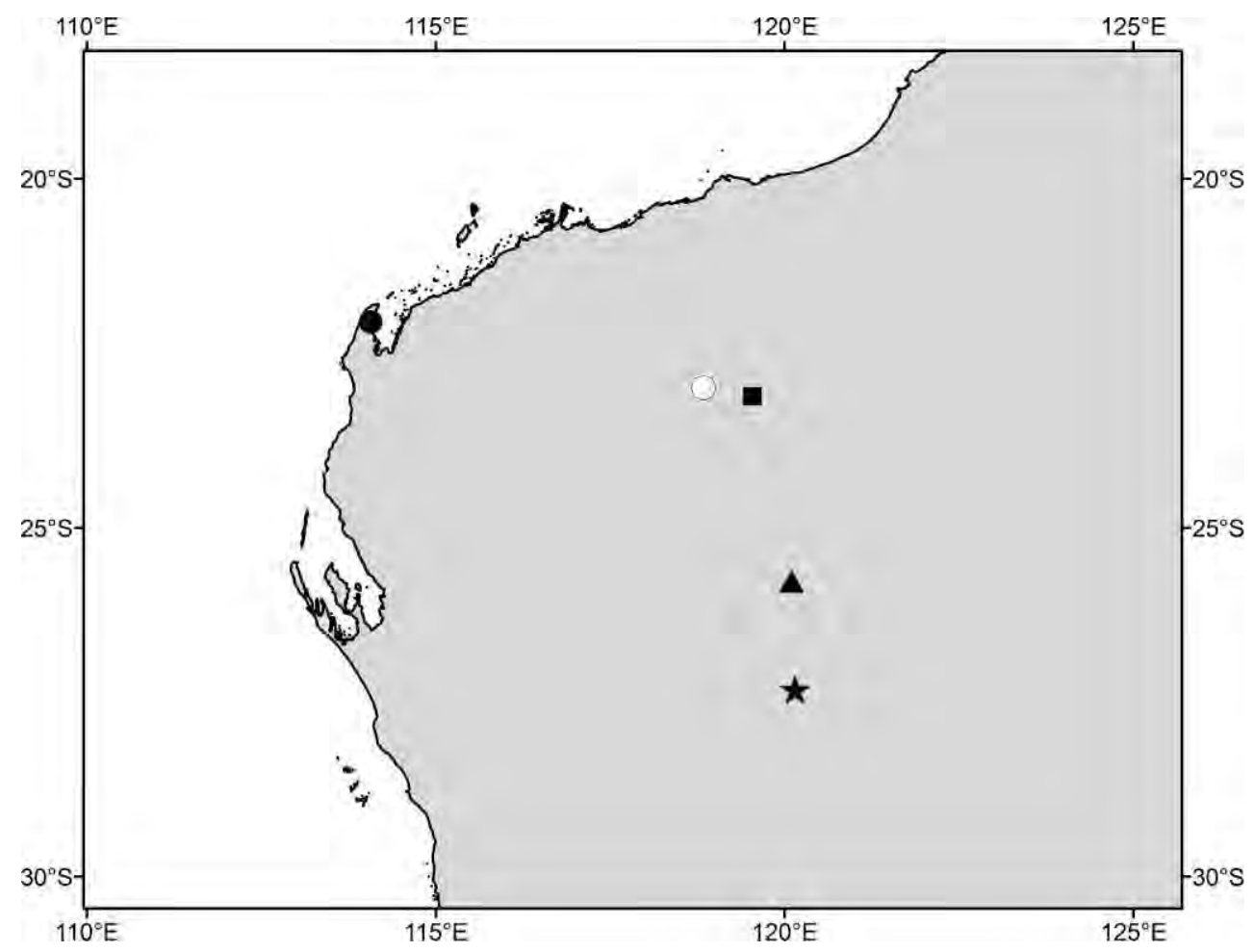

Map 10. Distribution records of Prethopalpus alexanderi $(\bullet)$, P. julianneae $(\bigcirc)$, P. cooperi $(\mathbf{\Delta})$, P. boltoni $(\square)$, and P. callani $(\star)$.

granulate, sides mostly smooth; lateral margin without denticles. Eyes: six, well developed but relatively small, ALE 0.040, PME 0.039, PLE 0.037; ALE largest, all eyes circular; posterior eye row straight from both above and front; ALE separated by less than their radius, ALE-PLE separated by less than ALE radius, PME just touching, PLE-PME separated by less than PME radius. Sternum longer than wide, surface smooth, without pits. Endites twice as long as wide, with tiny anteromedian projection. Abdomen cylindrical; book lung covers large hardly visible; pedicel tube short, ribbed. Dorsal scutum yellow, covering full length of abdomen, middle surface smooth, sides smooth; epigastric scutum not protruding; postepigastric scutum long, almost rectangular, covering nearly full length of abdomen. Legs yellow, patella plus tibia I shorter than carapace. Palpal femur attaching to patella subbasally; patella about as long as femur, ventrally slightly bulging, and basally rounded, bulb pyriform with thin, medially bent embolus (figs. 128-130).

Female (PBI_OON 23403, figs. 653659): Total length 1.22. Carapace, sides are slightly granulate, but cephalic area is smooth. Eyes: ALE 0.039, PME 0.038, PLE 0.036. Epigastric area with small epigynal opening, internal genital structure long oval, reaching posterior fold and ending in $\mathrm{T}$ shaped anterior sclerite (figs. 157-158).

OTHER MATERIAl EXAMINED: AUSTRALIA: Queensland: $16 \mathrm{~km} \mathrm{~N}$ of Pilgrim Sands (NQ 23), $16^{\circ} 0000 \mathrm{~S}, 145.4333^{\circ} \mathrm{E}, 28$ Nov. 1992-18 Apr. 1993, P. Lawless, R. Raven, M. Shaw, 1 of (QM S25118, PBI_OON 00021446); Mt Cook, Quarantine Road (NQ 21), $15.5000^{\circ} \mathrm{S}, 145.2333^{\circ} \mathrm{E}, 20 \mathrm{July}-28$ Nov. 1992, R. Raven, P. Lawless, M. Shaw, 1 ș, 2 + (QM S24421, PBI_OON 00020049); $3 \mathrm{~km}$ NNE of Mt Webb, $15.0500^{\circ} \mathrm{S}$, $145.1500^{\circ}$ E, 30 Apr.-3 May 1981, A. Calder, J. 
Feehan, 1 s, 2 q (ANIC 723, PBI_OON 00023404); Pilgrim Sands, $16.0833^{\circ} \mathrm{S}, 145.4333^{\circ} \mathrm{E}$, 20 July-28 Nov. 1992, R. Raven, P. Lawless, M. Shaw, 1 s , 1 우 (QM S24462, PBI_OON 00020941); $14.4 \mathrm{~km} \mathrm{~N}$ of Wudji Wudji, $15.86666^{\circ} \mathrm{S}, 135.31666^{\circ} \mathrm{E}, 4$ Nov. $^{1991-20}$ July 1992, P. Lawless, R. Raven, M. Shaw, 5 §ิ, 2 q (QM S24398, PBI_OON 00019930). PAPUA NEW GUINEA: National Capital District: Port Moresby, $9.46630^{\circ} \mathrm{S}$, 147.13610 E, 1 Aug. 1973 , J. Nieminen, 1 s , 1 + (UTU, PBI_OON 00015921); Port Moresby, $9.46630^{\circ} \mathrm{S}, 147.13610^{\circ} \mathrm{E}, 22$ Sep. 1973 , J. Nieminen, 2 ô, 2 ㅇ (UTU, PBI_OON 00015926).

DisTribution: This species is found in North Queensland and southern Papua New Guinea (map 7).

Prethopalpus oneillae Baehr and Harvey, new species

Figures 660-671; map 8

Type: AUSTRALIA: Western Australia: Male holotype from Homestead, $77.7 \mathrm{~km}$ NNW of Tom Price, borehole RHWRC0249P2 1, 22 $04^{\prime} 42^{\prime \prime} \mathrm{S}, 117^{\circ} 25^{\prime} 31^{\prime \prime} \mathrm{E}$ (29 Mar. 2010, C. O'Neill), deposited in WAM (T102800, PBI_OON 46537).

ETYMOLOGY: This species is named for Caitlin O'Neill, collector of the holotype.

Diagnosis: Males resemble those of $P$. alexanderi and $P$. cooperi in having a dorsal scutum covering the whole abdomen and no eyes, but can be separated from these species by the ovoid abdomen (fig. 660), and the slightly bulging epigastric area (in lateral view) (fig. 662).

MALE (PBI_OON 46537, figs 660-671): Total length 1.35. Prosoma, mouthparts, palp, and abdominal scuta pale orange, carapace broadly oval in dorsal view, pars cephalica flat in lateral view, surface granulate, only center of carapace smooth. Clypeus curved downward in front view, sloping forward in lateral view. Sternum fused to carapace, surface finely punctuate. Endites twice as long as wide. Abdomen ovoid; book lung covers large; pedicel ribbed. Dorsal scutum weakly sclerotized, covering full length of abdomen, no soft tissue visible from above, surface smooth; epigastric scutum slightly protruding; postepigastric scutum weakly sclerotized, long, almost rectangular, covering nearly full length of abdominal length, fused to epigastric scutum, without posteriorly directed lateral apodemes. Legs: white; patella plus tibia I shorter than carapace. Genitalia: epigastric region with sperm pore small, circular, situated in front of anterior spiracles, slightly protruding. Palpal patella ventrally bulging with rounded base; bulb ventrally bulging with short curled embolar tip (figs. 669-671).

Female: Unknown.

DisTRIBUTION: This species is known only from a single subterranean location in the Pilbara region of Western Australia (map 8).

Prethopalpus platnicki Baehr and

Harvey, new species

Figures 116-118, 159, 160, 672-690; map 7

TyPes: AUSTRALIA: Queensland: Male holotype from Thursday Island, Torres Strait, $10.58333^{\circ} \mathrm{S}, 142.2167^{\circ} \mathrm{E}$ (30 Mar.-15 June 1991, A. Von Berkey), deposited in QM (S79606, PBI_OON 00021352). Allotype female, collected with holotype, deposited in QM (S84698, PBI_OON 00023402).

ETYMOLOGY: This species is named for Norman Platnick in honor of his contribution to systematic theory, arachnid systematics, and the progenitor of the PBI project by which this research was funded.

Diagnosis: Males resemble those of $P$. attenboroughi in general body shape and bulbal shape, but can be separated by their strongly reduced eyes (fig. 677), and a patch of strong setae at the front of the carapace (fig. 675).

Male (PBI_OON 21352, figs. 672-681): Total length 1.05. Prosoma, mouthparts, and abdominal scuta yellow, carapace broadly oval in dorsal view, surface of elevated portion of pars cephalica smooth, sides finely reticulate; lateral margin without denticles; a field of stiff setae at cephalic area. Eyes reduced to 6 pale, hardly visible spots. Sternum longer than wide, surface smooth, without pits. Abdomen cylindrical; book lung covers large; pedicel tube short, ribbed. Dorsal scutum covering full length of abdomen, smooth; epigastric scutum not protruding; postepigastric scutum long, almost rectangular, covering nearly full length of abdomen. Legs white; patella plus tibia I 
nearly as long as carapace. Epigastric region with circular sperm pore. Palp with proximal segments yellow; embolus light; femur attaching to patella subbasally; patella longer than femur, slightly larger than femur; cymbium with distal patch of setae, not fused with bulb; embolus with massive, medially bent hook (figs. 116-118).

Female (PBI_OON 23402, figs. 682690): Total length 1.20. Carapace, pars cephalica without a field of setae. Eyes: six, pale, hardly visible spots. Epigastric area with large oval epigynal opening, internal genital structure large oval reaching posterior fold, anterior sclerite reduced to a short trunk (figs. 159, 160).

Other MAterial ExAmined: None.

DistribUtion: This species is known only from the type locality in the Torres Strait (map 7).

\section{Prethopalpus pearsoni Baehr and} Harvey, new species

Figures 691-700; map 8

TyPe: AUSTRALIA: Western Australia: Male holotype from mining Area C, bore PSD115R, c. $100 \mathrm{~km} \mathrm{NW}$ of Newman, $22^{\circ} 54^{\prime} 07.1^{\prime \prime} \mathrm{S}, \quad 118^{\circ} 59^{\prime} 19.2^{\prime \prime} \mathrm{E}, \quad$ troglofauna, -42m (10 Apr. 2010, G.B. Pearson, M.K. Curran), deposited in WAM (T108277, PBI_OON 46591).

ETYMOLOGY: This species is named for Grant Pearson, collector of the holotype.

DiAGNOSIS: Males can be separated from those of all other blind Prethopalpus species with a dorsal scutum by a larger distance between coxae II-III than between coxae I-II and II-IV (fig. 695).

Male (PBI_OON 46591, figs. 691-700): Total length 1.05. Prosoma, mouthparts, legs, palp, and abdominal scuta yellow pale orange, carapace elongate oval in dorsal view, pars cephalica flat in lateral view, surface smooth. Clypeus curved downward in front view, sloping forward in lateral view. Sternum longer than wide, not fused to carapace, surface smooth, distance between coxae II and III greater than distance between coxae I and II, and coxae III and IV, lateral margins with extension only between coxae II and III, leg I and II directed forward, with lateral pointed extensions, largest between coxae II and III. Endites twice as long as wide, directed medially. Abdomen cylindrical. Dorsal scutum weakly sclerotized covering more than $3 / 4$ of abdomen, more than $1 / 2$ to most of abdomen width, surface smooth, scutum barely visible; epigastric scutum not protruding; postepigastric scutum weakly sclerotized, long, semicircular, covering about $2 / 3$ of abdominal length, fused to epigastric scutum, without posteriorly directed lateral apodemes; spinneret scutum absent. Legs: patella plus tibia I shorter than carapace. Genitalia: epigastric region with sperm pore small, circular, situated at level of anterior spiracles. Palpal patella basally rounded ventrally only slightly bulging; bulb ventrally rounded with narrow embolar tip (figs. 698-700).

FEMALE: Unknown.

Other Material Examined: None.

DisTRIBUTION: This species is known only from subterranean habitats in the Pilbara region of Western Australia (map 8).

Prethopalpus rawlinsoni Baehr and Harvey, new species

Figures 122-124, 701-708; map 6

TyPe: AUSTRALIA: Queensland: Male holotype from Boomer Range, Python Scrub, site $5,23.18983^{\circ} \mathrm{S}, 149.7245^{\circ} \mathrm{E}, 240 \mathrm{~m}(16$ Dec. 1999-22 Mar. 2000, G. Monteith), deposited in QM (S78688, PBI_OON 00007129).

ETYMOLOGY: The specific name is a patronym in honor of Wendy Hebron's father, a great naturalist. Wendy Hebron, technician at the Queensland Museum, prepared all of the scientific drawings for this paper and obtained this skill from her father.

Diagnosis: Males resemble those of $P$. attenboroughi in having a strongly protruding epigastric scutum and a ventrally bulging bulb, but can be separated by the bulb having a conical extension (figs. 122, 124).

Male (PBI_OON 7129, figs. 701-708): Total length 1.24. Prosoma, mouthparts, and abdominal scuta pale orange, surface of elevated portion of pars cephalica smooth, sides granulate; lateral margin without denticles. Eyes: ALE 0.052; PME 0.034; PLE 0.045; ALE largest, ALE circular, PME squared, PLE circular; posterior eye row 
straight from both above and front; ALE separated by less than their radius, ALE-PLE separated by less than ALE radius, PME just touching, PLE-PME separated by less than PME radius. Sternum longer than wide, surface smooth, with pits. Endites twice as long as wide, with tiny toothlike projection. Abdomen cylindrical; book lung covers large, slightly darker that scutum; pedicel tube short, ribbed. Dorsal scutum, covering full length of abdomen, smooth; epigastric scutum strongly protruding; postepigastric scutum long, almost rectangular, covering nearly full length of abdomen, with long posteriorly directed lateral apodemes. Legs white; patella plus tibia I nearly as long as carapace. Epigastric region with circular sperm pore. Palp pale orange; embolus light; femur attaching to patella submedially; patella longer than femur, enlarged; cymbium not fused with bulb, bulb ventrally with large basal conical extension; embolus poorly differentiated, blunt (figs. 122-124).

Female: Unknown.

Other MATERIAl ExAmined: AUSTRALIA: Queensland: Boomer Range, Mongrel Scrub, site $1,23.19317^{\circ} \mathrm{S}, 149.7687^{\circ} \mathrm{E}, 200 \mathrm{~m}, 16$ Dec. 1999-22 Mar. 2000, G. and S. Monteith, 1 s (QM S79628, PBI_OON 00021478).

DistRIBUTION: This species is known only from eastern Queensland (map 6).

\section{Prethopalpus scanloni Baehr and Harvey, new species \\ Figures 709-718; map 8}

TyPe: AUSTRALIA: Western Australia: Male holotype from Robe River Valley, c. $65 \mathrm{~km}$ SW of Pannawonica, bore DBRC089, $21^{\circ} 44^{\prime} 00.4^{\prime \prime} \mathrm{S}, \quad 115^{\circ} 45^{\prime} 41.9^{\prime \prime} \mathrm{E}, \quad$ troglofauna, -9m (22 Sept. 2010, J.S. Cocking, M.D. Scanlon), deposited in WAM (T108284, PBI_OON 46598).

ETYMOLOGY: This species is named for Michael Scanlon, collector of the holotype.

DiAgNosis: Males can be separated from those of all other blind Prethopalpus species without a dorsal scutum by having a strongly bulging epigastric area (in lateral view) (fig. 711).

Male (PBI_OON 00046598, figs. 709718): Total length 1.16. Prosoma, mouthparts, legs, palp, and abdominal scuta pale orange, carapace ovoid in dorsal view, pars cephalica flat in lateral view, surface smooth. Clypeus straight in front view, sloping forward in lateral view. Sternum as long as wide, not fused to carapace, surface smooth, distance between coxae approximately equal, lateral margins unmodified. Chelicerae anterior face with swelling. Endites twice as long as wide, directed medially. Abdomen ovoid; book lung covers large; pedicel unmodified. Dorsal scutum absent; epigastric scutum surrounding pedicel, not protruding; postepigastric and spinneret scuta absent. Legs: patella plus tibia I near as long as carapace. Genitalia: epigastric region with sperm pore small, circular, situated at level of anterior spiracles. Sperm pore slightly protruded; patella basally narrow, ventrally only slightly bulging; bulb ventrally rounded with narrow embolar tip (figs. 716-718).

FeMale: Unknown.

Other MATERIAl Examined: None.

DISTRIBUTION: This species is known only from subterranean environments in the Robe River Valley in the Pilbara region of Western Australia (map 8).

\section{Prethopalpus tropicus Baehr and Harvey, new species}

Figures 125-127, 155, 156, 719-749; map 6

TyPES: AUSTRALIA: Queensland: Male holotype from $\mathrm{Mt}$ Windsor Tableland, $16.1700^{\circ} \mathrm{S}, 145.5000^{\circ} \mathrm{E}, 900 \mathrm{~m}$ (5 November 1976, R.W. Taylor and A. Weir), deposited in ANIC (PBI_OON 00023050). Female allotype collected with holotype (deposited in ANIC, PBI_OON 00023373).

ETYMOLOGY: The specific name refers to the occurrence of this species in the tropical region of North Queensland and Papua New Guinea.

DiAgNosis: Males and females resemble those of $P$. marionae in general body shape and eye sizes, but can be separated by a finely reticulate sternal surface (figs. 720, 735, 745).

Male (PBI_OON 23050, figs. 719-740): Total length 1.27. Prosoma, mouthparts, and abdominal scuta pale orange, pars cephalica flat in lateral view, surface of elevated portion of pars cephalica smooth, sides granulate; lateral margin without denticles. Eyes: six, small, ALE 0.040, PME 0.020, PLE 
0.018; ALE largest, ALE circular, PME oval, PLE circular; posterior eye row straight from both above and front; ALE separated by their radius to diameter, ALE-PLE separated by less than ALE radius, PME separated by their radius to diameter, PLE-PME separated by less than PME radius. Sternum longer than wide, surface finely reticulate, without pits. Chelicerae paturon distal region abruptly narrowed. Labium triangular, fused to sternum; with 3-5 setae on anterior margin. Endites serrula with one row of low blunt stubs and some additional stubs laterally (fig. 728); endites twice as long as wide, toothlike projection tiny. Abdomen cylindrical; book lung covers large; pedicel tube short, ribbed. Dorsal scutum covering full length of abdomen, punctuate; epigastric scutum not protruding; postepigastric scutum long, semicircular, covering nearly full length of abdomen. Legs pale orange; patella plus tibia I shorter than carapace. Epigastric region with sperm pore oval, rebordered. Palp pale orange; embolus dark; femur attaching to patella subbasally; patella about as long as femur, enlarged; cymbium not fused with bulb, bulb pyriform with long thin, medially bent embolus (figs. 125-127).

Female (PBI_OON 23373, figs. 741749): Total length 1.64. Eyes: ALE 0.032, PME 0.019, PLE 0.018. Epigastric area with small oval epigynal opening, internal genital structure short oval, not reaching posterior fold with $\mathrm{T}$-shaped anterior structure (figs. 155, 156).

Other MATERial ExAmined: AUSTRALIA: Queensland: Alexandra Bay, $16.18333^{\circ} \mathrm{S}, 145.45^{\circ} \mathrm{E}, 21$ June 1971 , Taylor and Feehan, 1 क (ANIC 328, PBI_OON 00022942); Black Mt Road, $5 \mathrm{~km} \mathrm{~N}$ of Kuranda, $16.78333^{\circ} \mathrm{S}, 145.65^{\circ} \mathrm{E}, 1200 \mathrm{~m}, 2$ Dec. 1988, G. Monteith, G. Thompson, 1 q (QM S58111, PBI_OON 00022266); Cape Tribulation, $5 \mathrm{~km} \mathrm{~W}$ (site 10$), 16.08333^{\circ} \mathrm{S}$, $145.4333^{\circ} \mathrm{E}, 780 \mathrm{~m}, 1-31$ Jan. 1983, G. Monteith, 1 ㅇ (QM S12700, PBI_OON 00022878); same location, 23 Apr. 1983, G. Monteith, D. Yeates, 1 \& (QM S12871, PBI_OON 00022877); Cape Tribulation, $16.60000^{\circ} \mathrm{S}, 154.27000^{\circ} \mathrm{E}, 50 \mathrm{~m}, 21$ June 1971, Taylor, Feehan, 1 오 (ANIC, PBI_OON 00022932); Cape Tribulation, $16.08333^{\circ} \mathrm{S}$, $145.4333^{\circ}$ E, 18 Mar. 2000, R. Kitching, 1 s
(ANIC, PBI_OON 00022971); same location, 20-29 July 1992, R.R. Forster, 1 i (QM S78175, PBI_OON 00022870); 2 km WNW of Cape Tribulation (site 2), $16.08333^{\circ} \mathrm{S}$, $145.4667^{\circ} \mathrm{E}, 50 \mathrm{~m}, 23$ Sep.-7 Oct. 1982, G. Monteith, D. Yeates, G. Thompson, 1 s (QM S12864, PBI_OON 00006426); Cape Tribulation, $16.08333^{\circ} \mathrm{S}, 145.4333^{\circ} \mathrm{E}, 4$ Nov. 1991-20 July 1992, P. Lawless, R. Raven, M. Shaw, 1 o (QM S21757, PBI_OON 00019966); Crystal Cascades, carpark, $16.96667^{\circ} \mathrm{S}, 145.6733^{\circ} \mathrm{E}, 23$ July-26 Nov. 1992, R. Raven, P. Lawless, E. Lawless, M. Shaw, 2 o, 1 ㅇ (QM S24014, PBI_OON 00020951); Danbulla State Forest, 1,000 m, 6 Aug. 1992, J. and F. Murphy, 1 के (AMNH 21350, PBI_OON 00036744); Danbulla State Forest, Edith Road, $4.3 \mathrm{~km} \mathrm{~N}$ of Danbulla Forest Drive, $17.1^{\circ} \mathrm{S}, 145.6^{\circ} \mathrm{E}, 875 \mathrm{~m}, 29 \mathrm{Aug}$. 2004, Thayer, Newton, 1 के (FMNH-INS 0000034 158, PBI_OON 00023372); Ella Bay, 7 Aug. 1992, J. and F. Murphy, 1 \& (AMNH 21384, PBI_OON 00036891); Hann Tableland (North end), $16.81667^{\circ} \mathrm{S}, 145.1833^{\circ} \mathrm{E}$, 950 m, 4 Aug.-9 Sep. 1988, Storey, Dickenson, 1 t (QM S78248, PBI_OON 00007232); Heberton Range State Forest, Rifle Range Road, $17.2871^{\circ} \mathrm{S}, 145.429^{\circ} \mathrm{E}$, 1260 m, 23 July 2004, Newton, Thayer, 1 s (FMNH-INS $0000 \quad 033$ 878, PBI_OON 00023339); Hughes Rd, Topaz, $17.43333^{\circ} \mathrm{S}$, $145.7167^{\circ} \mathrm{E}, 650 \mathrm{~m}, 1 \mathrm{Sep} .1993$, G. Monteith, S. Breeden, 1 के (QM S23061, PBI_OON $00021470) ; 40 \mathrm{~km} \mathrm{~W}$ of Ingham near Wallaman Falls, $18.60000^{\circ} \mathrm{S}, 145.800000^{\circ} \mathrm{E}, 600 \mathrm{~m}$, 7 Aug. 1982, litter, J. Peck, 1 of (FMNH-NFINS 0000061 607, PBI_OON 00010893); Kirrama Range, Douglas Creek Rd., 800 m, 26-30 Aug. 1987, R. Raven, 1 के (QM S80972, PBI_OON 00022768); Lake Barrine, 1000 m, 8 Aug. 1992, J. and F. Murphy, 2 s (AMNH 21435, PBI_OON 00036892); Longlands Gap State Forest (NQ 13), $17.4550^{\circ} \mathrm{S}$, $145.4742^{\circ}$ E, 23 July-26 Nov. 1992, R. Raven, P. Lawless, E. Lawless, M. Shaw, 1 to, 1 우 (QM S27917, PBI_OON 00023336); McDowall Range, $17 \mathrm{~km} \mathrm{~N}$ of Daintree, $16.1000^{\circ} \mathrm{S}$, $145.3333^{\circ} \mathrm{E}, 520 \mathrm{~m}, 27$ Nov. 1985, G. Monteith, 1 s (QM S72974, PBI_OON 00020018); McNamee Creek, $17.4000^{\circ} \mathrm{S}, 145.4800^{\circ} \mathrm{E}$, 400 m, 8 July 1971, Taylor, Feehan, 1 (ANIC, PBI_OON 00022995); Mossman, $16.47000^{\circ} \mathrm{S}, 145.3772^{\circ} \mathrm{E}, 11 \mathrm{Jul} .1982$, rainforest, 
litter, J. Peck, 1 ơ (FMNH-NF-INS 0000061 603PBI_OON 00023536); Mossman Bluff Track, 5-10 km W of Mossman (Site 7), $16.46667^{\circ} \mathrm{S}, 145.3667^{\circ} \mathrm{E}, 16-30$ Dec. $1988, \mathrm{G}$. Monteith, G. Thompson, ANZSES Expedition, 1 sै (QM S21731, PBI_OON 00019960); Mossman Bluff Track, 5-10 km W of Mossman (Site 7), $16.46667^{\circ} \mathrm{S}, 145.3667^{\circ} \mathrm{E}, 1000 \mathrm{~m}$, 20 Dec. 1989-15 Jan. 1990, G. Monteith, G. Thompson, ANZSES Expedition, 1 के (QM S41544, PBI_OON 00006971); Mossman Gorge National Park (NQ 15), $16.47167^{\circ} \mathrm{S}$, $145.3333^{\circ} \mathrm{E}, 1$ Nov. 1991-22 July 1992, P. Lawless, R. Raven, M. Shaw, 2 के (QM S69216, PBI_OON 00007340 (QM S69218, PBI_OON 00020934); Mt Bartle-Frere, E slope, $17.38333^{\circ} \mathrm{S}, 145.7667^{\circ} \mathrm{E}, 17$ June 1969 , A. Walford-Higgins, 1 + (ANIC, PBI_OON 00022933); Mt Bartle-Frere, W base, $17.38333^{\circ} \mathrm{S}, \quad 1^{2} 45.7667^{\circ} \mathrm{E}, 700 \mathrm{~m}, 25$ Nov. 1994-10 Jan. 1995, G. Monteith, J. Hasenpusch, 1 के (QM S37866, PBI_OON 00020680); Mt Boolbun Sth (Creek), $15.9500^{\circ} \mathrm{S}$, $145.1333^{\circ} \mathrm{E}, 800 \mathrm{~m}, 5$ Nov. 1995-11 Jan. 1996, G. Monteith, D. Cook, L. Roberts, 7 \& (QM S44573, PBI_OON 00006786); Mt Cook, Quarantine Road (NQ 21), $15.5000^{\circ} \mathrm{S}$, 145.2333 ${ }^{\circ}$, 28 Nov. 1992-18 Apr. 1993, R.J. and S. Raven, P. and E. Lawless, 1 is (QM S36177, PBI_OON 00007008); Mt Demi, 7 km SW of Mossmann, $16.5000^{\circ} \mathrm{S}, 145.3167^{\circ} \mathrm{E}$, 1100 m, 29 Oct. 1983, D.K. Yeates, I. Thompson, 1 q (QM S79662, PBI_OON 00021532); Mt Demi Nth Peak, $16.5000^{\circ} \mathrm{S}, 145.3167^{\circ} \mathrm{E}, 1050 \mathrm{~m}$, 17 Dec. 1995-25 Jan. 1996, G. Monteith, G. Thompson, Ford, 2 s (QM S41526, PBI_OON 00007186; QM S43391, PBI_OON 00006758); Mt Haig, $17.1000^{\circ} \mathrm{S}, 145.6000^{\circ} \mathrm{E}, 150 \mathrm{~m}, 29$ Oct. $^{\circ}$ 1976, R.W. Taylor, T.A. Weir, 2 \& (ANIC, PBI_OON 00022978); Mt Lewis, $16.35000^{\circ} \mathrm{S}$, $145.17000^{\circ} \mathrm{E}, 960 \mathrm{~m}, 30$ Oct. 1976; R.W. Taylor, T.A. Weir, 1 ㅇ (ANIC, PBI_OON 00023047); Mt Lewis, $2.5 \mathrm{~km} \mathrm{~N}, 16.56667^{\circ} \mathrm{S}, 145.2667^{\circ} \mathrm{E}$, 1040 m, 3 Nov. 1983, D. Yeates, G. Thompson, 2 \& (QM S16804, PBI_OON 00022875); Mt Tiptree, $17.0666^{\circ} \mathrm{S}, 145.616^{\circ} \mathrm{E}, 900 \mathrm{~m}, 29$ June 1971, Taylor, Feehan, 2 q (ANIC, PBI_OON 00023041); Mt Windsor Tableland, $16.1700^{\circ} \mathrm{S}, 145.5^{\circ} \mathrm{E}, 900 \mathrm{~m}, 5$ Nov. $1976, \mathrm{R} . \mathrm{W}$. Taylor, T.A. Weir, 1 ㅇ (ANIC, PBI_OON 00022979); Mt Windsor State Forest, $1 \overline{6} .25^{\circ} \mathrm{S}$, $145.03333^{\circ} \mathrm{E}, 1060 \mathrm{~m}, 1$ Sep. 2004, Thayer, Newton, 6 ㅇ (FMNH-INS 0000034 106,
PBI_OON 00023340); Mt Windsor Tableland, $16.1700^{\circ} \mathrm{S}, 145.5000^{\circ} \mathrm{E}, 900 \mathrm{~m}, 5$ Nov. 1976 , R.W. Taylor and A. Weir, 1 s, 1 ㅇ (ANIC 552, PBI_OON 00022974); Mt Windsor Tableland, Whypalla State Forest (pitfalls 11, 12), $16.25717^{\circ} \mathrm{S}, 145.038^{\circ} \mathrm{E}, 1060 \mathrm{~m}$, Dec. 1992-01 Dec. 1993, S. Burnett, 3 के (QM S33155, PBI_OON 00019935); same data 2 sे, 1 ㅇ (QM S24538, PBI_OON 00020978); Mt Windsor Tableland, Whypalla State Forest, pitfall \#1-4, $16.27533^{\circ} \mathrm{S}, 145.0672^{\circ} \mathrm{E}, 1180 \mathrm{~m}$, Dec. 1992-01 Dec. 1993, S. Burnett, 3 के (QM S33122, PBI_OON 00019928; QM S33139, PBI_OON 00019995); Pilgrim Sands, $16.7000^{\circ} \mathrm{S}, \quad 145.4667^{\circ} \mathrm{E}, 19$ July-29 Nov. 1992, R. Raven, P. and E. Lawless, M. Shaw, 1 q (QM S24557, PBI_OON 00020957); Pilgrim Sands (NQ 25), $16.7000^{\circ} \mathrm{S}$, $145.4667^{\circ} \mathrm{E}, 5$ Nov. $1991-20$ July $1992, \mathrm{R}$. Raven, P. Lawless, M. Shaw, 3 is (QM S24871, PBI_OON 00021432); Ravenshoe, $20 \mathrm{~km} \mathrm{~S}, 17.81666^{\circ} \mathrm{S}, 145.53333^{\circ} \mathrm{E}, 3$ July 1971, Taylor and Feehan, 3 s, 2 ㅇ (ANIC 358, PBI_OON 00023031); Roaring Meg Creek, $6 \mathrm{~km} \mathrm{~W}$ of Cape Tribulation, $16.06667^{\circ} \mathrm{S}$, $145.4^{\circ} \mathrm{E}, 740 \mathrm{~m}, 5$ Oct. 1982, G. Monteith, D. Yeates, G. Thompson, 1 o (QM S12815, PBI_OON 00006542); Russell River National Park, $17.30833^{\circ} \mathrm{S}, 145.9625^{\circ} \mathrm{E}, 30$ Oct. $1991-24$ June 1992, P. Lawless, R. Raven, M. Shaw, 3 §., 2 ㅇ (QM S19924, PBI_OON 00019926); Shiptons Flat, $15.8000^{\circ} \mathrm{S}, 145.2500^{\circ} \mathrm{E}, 250 \mathrm{~m}$, 17-19 Oct. 1980, T.A. Weir, 3 s , 3 ㅇ (ANIC, PBI_OON 00023055); The Crater National Park, $17.42167^{\circ} \mathrm{S}, \quad 145.4858^{\circ} \mathrm{E}, 23$ July-26 Nov. 1992, R. Raven, P. Lawless, E. Lawless, M. Shaw, 2 s, 2 우 (QM S22578, PBI_OON 00021455); same location, 26 Nov. 1992-15 Apr. 1993, R.J. and S. Raven, P. and E. Lawless, 1 के (QM S19827, PBI_OON 00007419); Tully Falls National Park, $17.47^{\circ} \mathrm{S}, 145.33^{\circ} \mathrm{E}, 2$ July 1971 , Taylor, Feehan, 1 (ANIC, PBI_OON 00022909); Windsor Tableland, $35 \mathrm{~km}$ NNW of Mt Carbine, $16.2500^{\circ} \mathrm{S}, 145.1333^{\circ} \mathrm{E}, 1050 \mathrm{~m}, 25$ Apr. 1982 , G. Monteith, D. Yeates, D. Cook, 2 \& (QM S79754, PBI_OON 00021576). Windsor Tableland, $35 \mathrm{~km} \mathrm{NNW}$ of Mt Carbine, 16.2500 ${ }^{\circ}$ S, $145.1333^{\circ} \mathrm{E}, 1050 \mathrm{~m}, 25$ Apr. 1982 , G. Monteith, D. Yeates, D. Cook, 1 s, 2 ㅇ (QM S16438, PBI_OON 00021538). PAPUA NEW GUINEA: National Capital District: Port Moresby, $9.5333^{\circ} \mathrm{S}, 147.15^{\circ} \mathrm{E}, 22$ Sep. 
1973, J. Nieminen, 2 ㅇ (UTU, PBI_OON 00015951). Central Province: Girinumu, Owers corner, 13 Feb.-22 Mar. 1974, P. Lehtinen, 1 के (UTU, PBI_OON 00015968); Haima, $9.43333^{\circ} \mathrm{N}, 147.2000^{\circ} \mathrm{E}, 12$ Feb. -22 Mar. 1974, P. Lehtinen, 6 s, 5 ㅇ (UTU, PBI_OON 00015954, PBI_OON 00015912); Laloki River, 12 Feb. 1974, P. Lehtinen, 1 o (UTU, PBI_OON 00015893); Motumotu-S, Goragatabu Creek, $9.2000^{\circ} \mathrm{N}, 147.23333^{\circ} \mathrm{E}$, 12 Feb.-22 Mar. 1974, P. Lehtinen, 3 के (UTU, PBI_OON 00015899); Sapphire, 9.41666 ${ }^{\circ} \mathrm{N}, 147.31666^{\circ} \mathrm{E}, 13$ Feb.-22 Mar. 1974, P. Lehtinen, 1 q (UTU, PBI_OON $00015857)$; Taurama, $7.41666^{\circ} \mathrm{N}, 144.26666^{\circ} \mathrm{E}$, 11 Feb.-21 Mar. 1974, P. Lehtinen, 1 o, 1 ㅇ (UTU, PBI_OON 00015940); same location, 25 Feb. 1974, P. Lehtinen, 1 ㅇ (UTU, PBI_OON 00015955); same location, 11 Feb.-21 Mar. 1974, P. Lehtinen, 5 s (UTU, PBI_OON 00015969).

DistribUtion: Prethopalpus tropicus occurs in North Queensland and Papua New Guinea (map 6).

\section{ACKNOWLEDGMENTS}

This paper was completed with support from the National Science Foundation's PBI (Planetary Biodiversity Inventory) program provided through grant DEB-0613754. We thank Jason Alexander and Garth Humphreys (Biota Environmental Sciences), Shae Callan and Stefan Eberhard (Subterranean Ecology), Christa Deeleman and I.J. Smit (National Natuurhistorische Museum, Leiden), Bruce Halliday (Australian National Insect Collection, Canberra), Yvonne KranzBaltensperger and Christian Kropf (Natural History Museum, Bern), Pekka Lehtinen (University of Turku), Jochen Martens (Senckenberg Museum, Frankfurt am Main), Jane McRae, and Stuart Halse (Bennelongia), Norman Platnick (American Museum of Natural History, New York), Robert Raven and Owen Seeman (Queensland Museum, Brisbane), Peter Schwendinger (Muséum d'Histoire Naturelle, Geneva), Petra Sierwald (Field Museum of Natural History, Chicago) and Julianne Waldock (Western Australian Museum, Perth) for loan of the material, and their support of the project. We thank Peter Schwendinger, Alexandre Bo- naldo, and Norman Platnick for their insightful comments on the manuscript, and Wendy Hebron for her excellent drawings.

\section{REFERENCES}

Baehr, B.C., and M.S. Harvey. 2010. Two new species of the endemic Australian goblin spider genus Cavisternum (Araneae: Oonopidae) from Queensland. Australian Entomologist 37: 171-177.

Baehr, B.C., and D. Ubick. 2010. The goblin spiders of the Asian genus Camptoscaphiella Caporiacco, 1934 (Araneae: Oonopidae). American Museum Novitates 3697: 1-65.

Baehr, B.C., M.S. Harvey, and H.M. Smith. 2010. A review of the new endemic Australian goblin spider genus Cavisternum (Araneae: Oonopidae). American Museum Novitates 3684: $1-40$.

Burger, M., W. Nentwig, and C. Kropf. 2002. Opopaea fosuma, n. sp. from Sumatra, Indonesia (Araneae, Oonopidae). Bulletin of the British Arachnological Society 12: 244-248.

Burger, M., W. Nentwig, and C. Kropf. 2003. Complex genital structures indicate cryptic female choice in a haplogyne spider (Arachnida, Araneae, Oonopidae, Gamasomorphinae). Journal of Morphology 255: 80-93.

Byrne, M., et al. 2008. Birth of a biome: insights into the assembly and maintenance of the Australian arid zone biota. Molecular Ecology 17: 4398-4417.

Fannes, W., and R. Jocqué. 2008. Ultrastructure of Antoonops, a new, ant-mimicking genus of Afrotropical Oonopidae (Araneae) with complex internal genitalia. American Museum Novitates 3614: 1-30.

Guzik, M.T., et al. 2011. Is the Australian subterranean fauna uniquely diverse? Invertebrate Systematics 24: 407-418.

Harvey, M.S. 1993. The systematics of the Hyidae (Pseudoscorpionida: Neobisioidea). Invertebrate Taxonomy 7: 1-32.

Harvey, M.S. 2001. New cave-dwelling schizomids (Schizomida: Hubbardiidae) from Australia. Records of the Western Australian Museum Supplement 64: 171-185.

Harvey, M.S. 2002. Short-range endemism in the Australian fauna: some examples from nonmarine environments. Invertebrate Systematics 16: $555-570$.

Harvey, M.S., and K.L. Edward. 2007a. A review of the pseudoscorpion genus Ideoblothrus (Pseudoscorpiones, Syarinidae) from western and northern Australia. Journal of Natural History 41: 445-472. 
Harvey, M.S., and K.L. Edward. 2007b. Three new species of cavernicolous goblin spiders (Araneae: Oonopidae) from Australia. Records of the Western Australian Museum 24: 9-17.

Harvey, M.S., and M.C. Leng. 2008. Further observations on Ideoblothrus (Pseudoscorpiones: Syarinidae) from subterranean environments in Australia. Records of the Western Australian Museum 24: 379-386.

Harvey, M.S., and E.S. Volschenk. 2007. The systematics of the Gondwanan pseudoscorpion family Hyidae (Pseudoscorpiones: Neobisioidea): new data and a revised phylogenetic hypothesis. Invertebrate Systematics 21: 365-406.

Harvey, M.S., O. Berry, K.L. Edward, and G. Humphreys. 2008. Molecular and morphological systematics of hypogean schizomids (Schizomida: Hubbardiidae) in semi-arid Australia. Invertebrate Systematics 22: 167-194.

Harvey, M.S., et al. 2011. Protecting the innocent: studying short-range endemic taxa enhances conservation outcomes. Invertebrate Systematics 25: 1-10.

Petrunkevitch, A. 1923. On families of spiders. Annals of the New York Academy of Sciences 29: 145-180.

Platnick, N.I. 2008. A new subterranean ground spider genus from Western Australia (Araneae: Trochanteriidae). Invertebrate Systematics 22: 295-299.

Platnick, N.I. 2011. The world spider catalog, version 12.0. New York: American Museum of Natural History, Internet resource (http:// research.amnh.org/iz/spiders/catalog), accessed August 21, 2011.
Platnick, N.I., and N. Dupérré. 2009a. The American goblin spiders of the new genus Escaphiella (Araneae, Oonopidae). Bulletin of the American Museum of Natural History 328: $1-151$.

Platnick, N.I., and N. Dupérré. 2009b. The goblin spider genera Opopaea and Epectris (Araneae, Oonopidae) in the New World. American Museum Novitates 3649: 1-43.

Platnick, N.I., and N. Dupérré. 2010a. The goblin spider genera Stenoonops and Australoonops (Araneae, Oonopidae), with notes on related taxa. Bulletin of the American Museum of Natural History 340: 1-111.

Platnick, N.I., and N. Dupérré. 2010b. The goblin spider genus Scaphiella (Araneae, Oonopidae). Bulletin of the American Museum of Natural History 332: 1-156.

Saaristo, M.I. 2001. Dwarf hunting spiders or Oonopidae (Arachnida, Araneae) of the Seychelles. Insect Systematics and Evolution 32: 307-358.

Saaristo, M.I., and Y.M. Marusik. 2008. A survey of African Opopaea Simon, 1891 (Arachnida, Aranei, Oonopidae). Arthropoda Selecta 17: 17-53.

Simon, E. 1893. Histoire naturelle des araignées. Vol. 1 (2), 2nd ed. Paris: Roret.

Wunderlich, J. 1994. Beschreibung bisher unbekanntner Spinnenarten und -Gattungen aus Malaysia und Indonesien (Arachnida: Araneae: Oonopidae, Tetrablemmidae, Telemidae, Pholcidae, Linyphiidae, Nesticidae, Theridiidae und Dictynidae). Beiträge zur Araneologie 4: 559-579. 


\section{INDEX OF SPECIFIC NAMES}

alexanderi, 80, map 10 attenboroughi, 80, map 6 bali, 30, map 2

bellicosus, 30, map 5 blosfeldsorum, 83, map 7 boltoni, 86, map 10 brunei, 32, map 2 callani, 89, map 10 cooperi, 91, map 10 deelemanae, 36, map 5 eberhardi, 92, map 9 fosuma, 39, map 2 framenaui, 93, map 9 humphreysi, 93, map 9 ilam, 12, map 1 infernalis, 95, map 8 java, 47, map 3 julianneae, 96, map 10 khasi, 11, map 1 kintyre, 97, map 9 kranzae, 50, map 3 kropfi, 51, map 5 leuser, 54, map 4 madurai, 14, map 1 mahanadi, 15, map 1 magnocularis, 58, map 5 maini, 97, map 8 marionae, 100, map 7 meghalaya, 25, map 1 oneillae, 105, map 8 pahang, 59, map 3 pearsoni, 106, map 9 perak, 61, map 3 platnicki, 106, map 7 rawlinsoni, 107, map 6 sabah, 63, map 4 sarawak, 64, map 4 scanloni, 107, map 8 schwendingeri, 67, map 4 tropicus, 108, map 6 utara, 72, map 5 